\title{
Functional Characterization of the Histone Methyltransferase and Methyl DNA Binding Protein MDU and its Role in Epigenetic Regulation of $R b f$ Gene in Drosophila melanogaster
}

\author{
Dissertation \\ zur Erlangung \\ des mathematisch-naturwissenschaftlichen Doktorgrades \\ "Doctor rerum naturalium" \\ der Georg-August-Universität Göttingen
}

\author{
Developmental Biology at the Institute \\ of Zoology, Anthropology and Developmental Biology, \\ Georg-August-Universität Göttingen \\ and \\ Department of Biochemistry, \\ University of California, Riverside
}
vorgelegt von
Dawei Gou

aus Xingcheng

2008 
Dekan:

Anleiter:

Referent:

Koreferent:

Eingereicht am:

Mündliche Prüfung am:
Prof. Dr. G. Braus

Prof. Sauer (University California, USA)

Prof. Wimmer

Prof. Kessel (MPI) 
Dissertation der Fakultät Naturwissenschaften der Georg-August-Universität Göttingen, erstellt extern am Department of Biochemistry der University of California Riverside.

Gutachter:

Prof. Dr. Ernst Wimmer (Georg-August-Universität Göttingen)

Prof. Dr. Frank Sauer (University of California Riverside) 
I herewith declare that, under supervision, I independently wrote the following thesis, using none other than the sources and aids listed. Also, during the thesis work, the principles and recommendations in "Verantwortung in der Wissenschaft" (Responsibility in Science) by the Georg-August-Universität Göttingen were observed.

September $5^{\text {th }}, 2008$

Date

Signature 
To my dear wife 


\section{Table of Contents}

I. TABLE OF CONTENTS

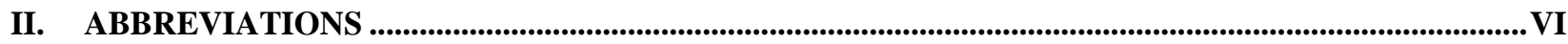

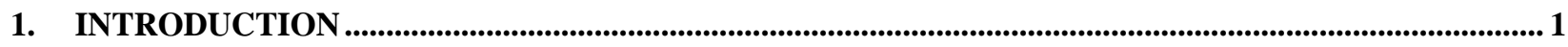

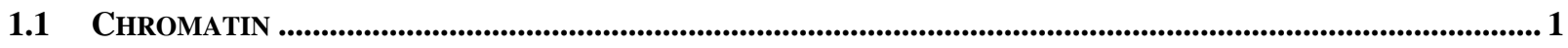

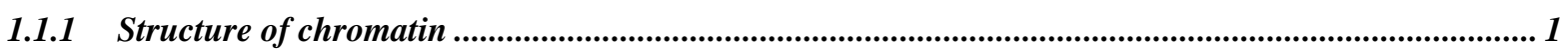

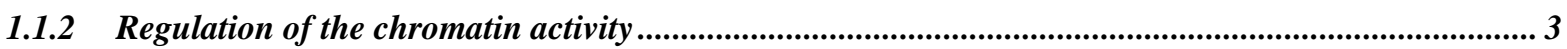

1.2 THE EPIGENETIC MODIFICATIONS OF DNA AND HISTONES......................................................................... 3

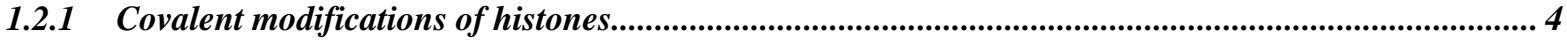

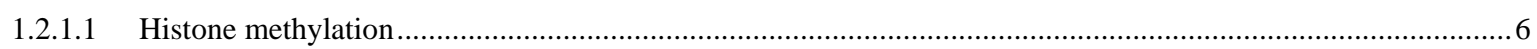

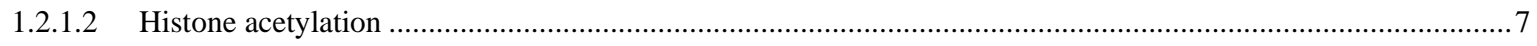

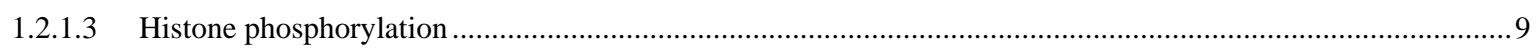

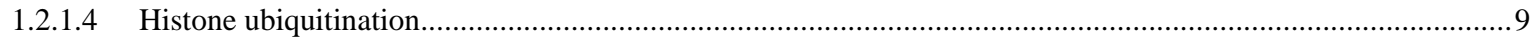

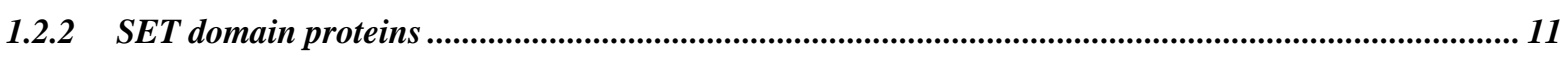

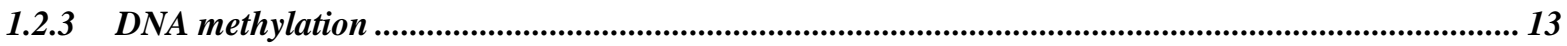

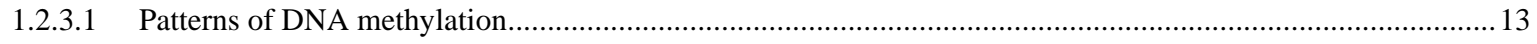

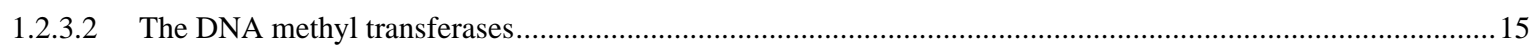

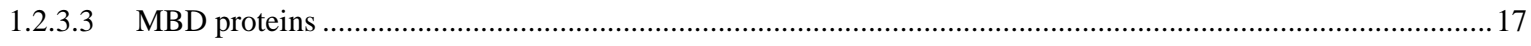

1.3 MBD/SET PROTEINS: BIFUNCTIONAL REGULATORS OF GENE EXPRESSION.................................................. 20

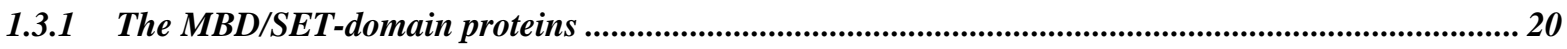

1.3.2 Gene structure and previous functional studies of $\mathrm{Mdu}$........................................................................ 20

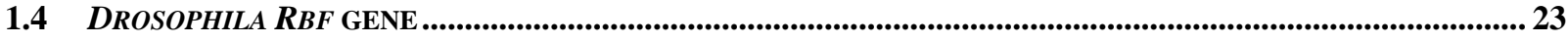

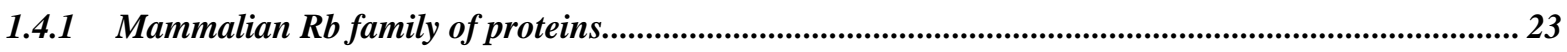

1.4.2 Function of "Retinoblastoma Family Proteins" in Drosophila ........................................................... 24

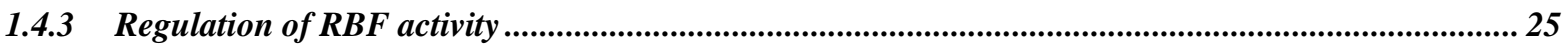

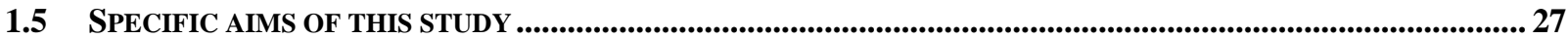

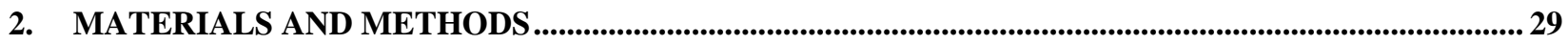

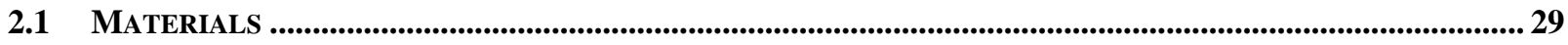

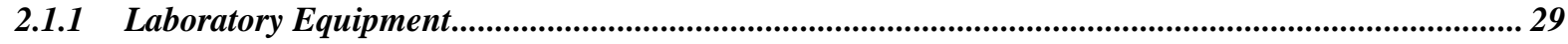

2.1.2 Consumables and Kits ....................................................................................................................... 30

2.1.3 Chemicals, Enzymes, Proteins and Molecular Weight Markers ........................................................... 31

2.1.4 Antibodies and Affinity Matrixes ......................................................................................................... 32

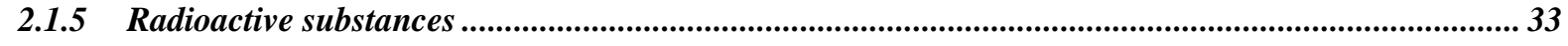

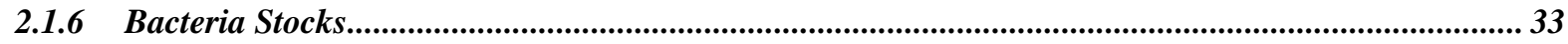




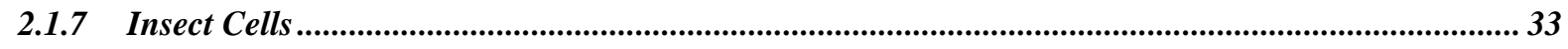

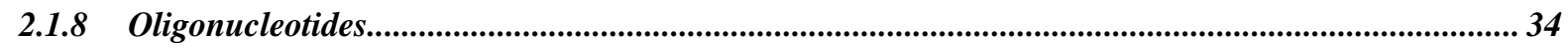

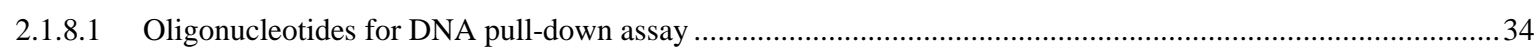

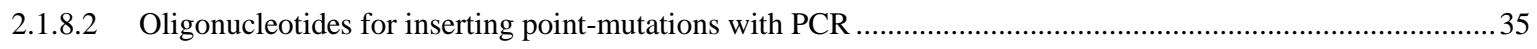

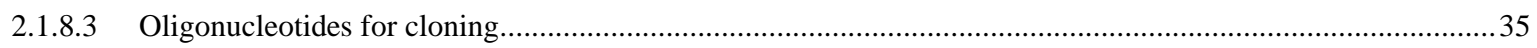

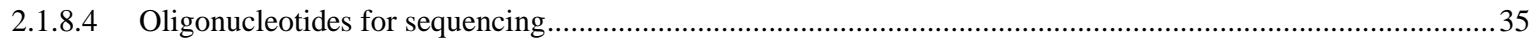

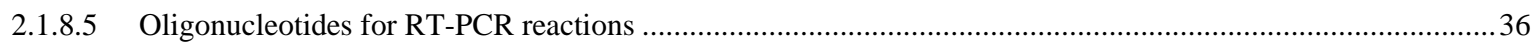

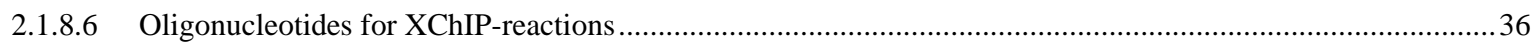

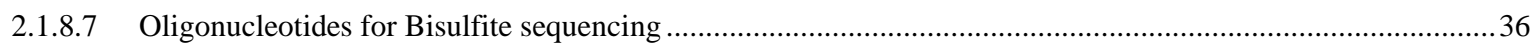

2.1.8.8 Oligonucleotides for PCR amplification after digestion with methylation sensitive enzymes............................37

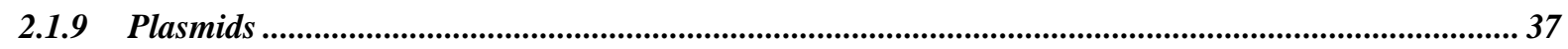

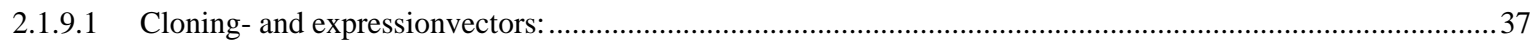

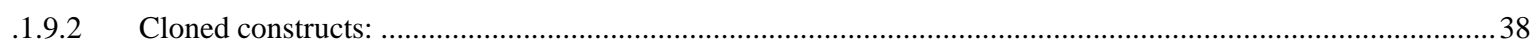

2.1.10 Baculoviruses for expression in Sf9-cellculture ........................................................................... 40

2.1.11 Media, Buffers and Stock Solutions............................................................................................. 41

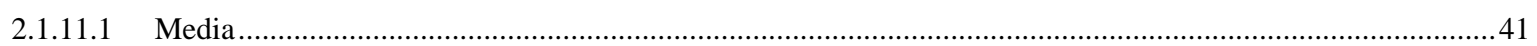

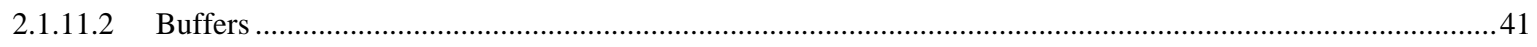

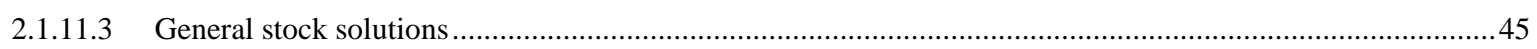

2.1.11.4 Protein and nondenaturing polyacrylamide gel solutions ...................................................................45

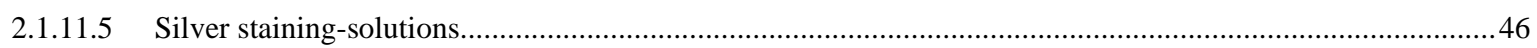

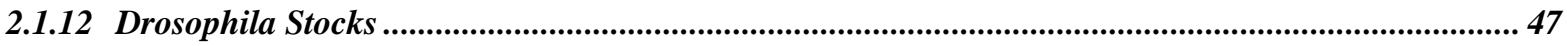

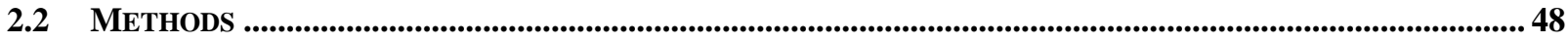

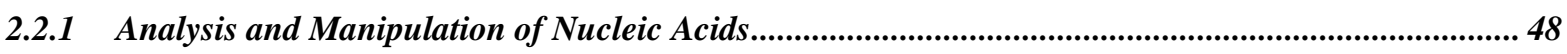

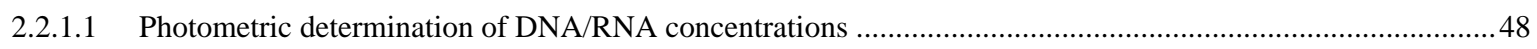

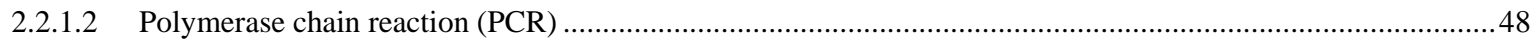

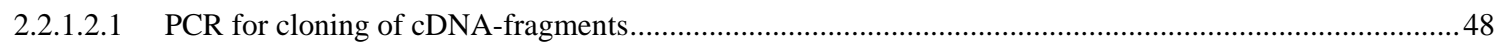

2.2.1.2.2 PCR for standard detection of specific DNA-sequences ...................................................................48

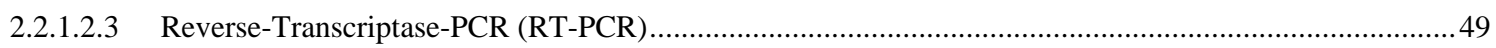

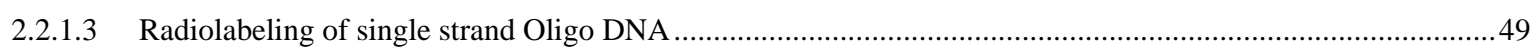

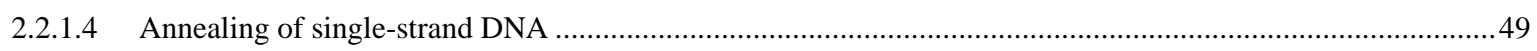

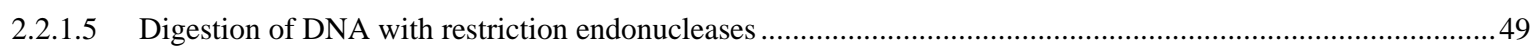

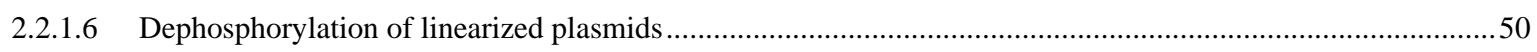

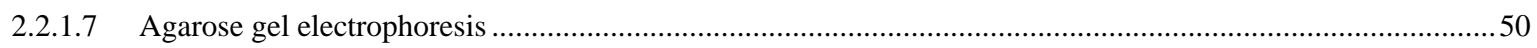

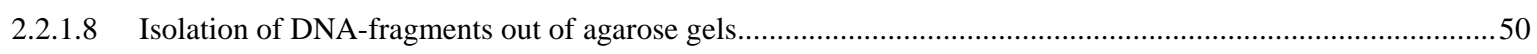

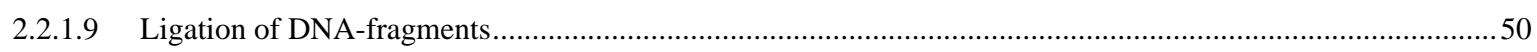

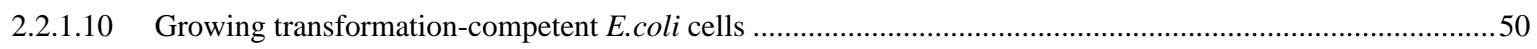

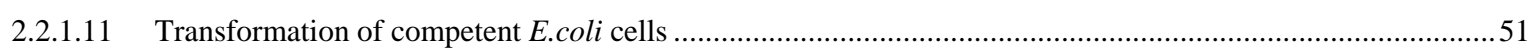

2.2.1.12 Isolation of plasmid-DNA out of E.coli cells............................................................................................51

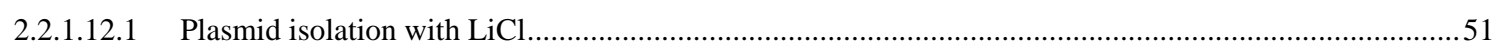

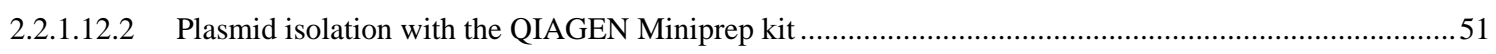

2.2.1.12.3 Plasmid isolation with the QIAGEN Maxiprep kit.................................................................................5 


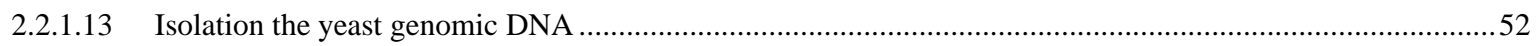

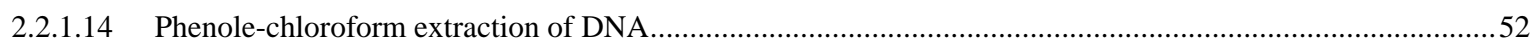

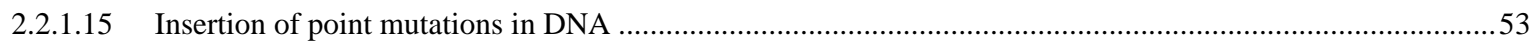

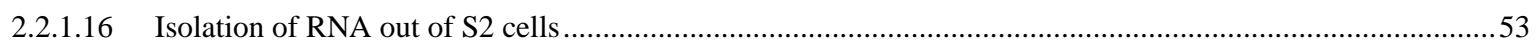

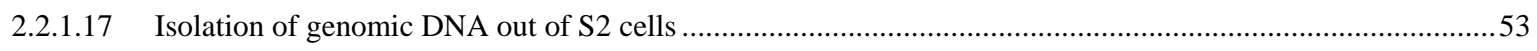

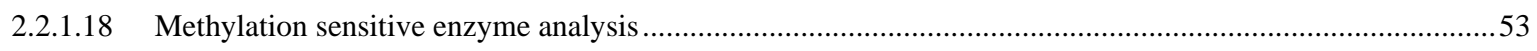

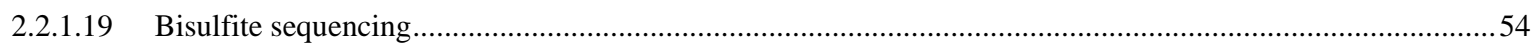

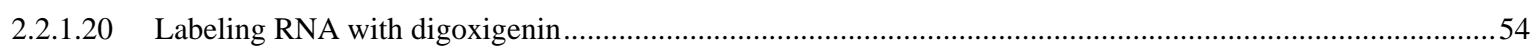

2.2.2 Analysis and Manipulation of Proteins..................................................................................... 55

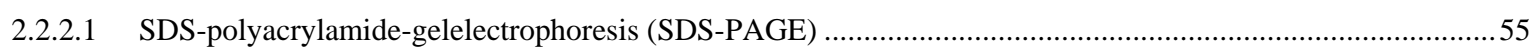

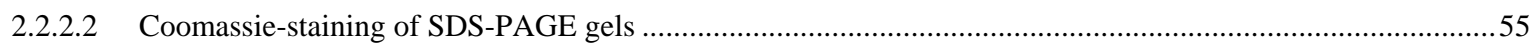

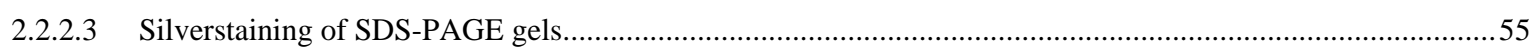

2.2.2.4 Immunodetection of SDS-PAGE proteins (Western-blot) …................................................................56

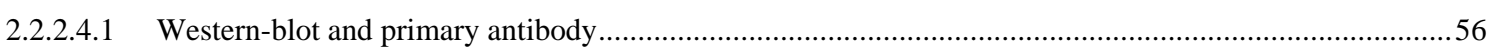

2.2.2.4.2 Immunodetection with alkaline phosphatase .................................................................................56

2.2.2.4.3 Immunodetection with chemiluminescence (ECL-Plus-Kit).................................................................5 56

2.2.3 Expression and affinity precipitation of recombinant Proteins out of E Coli .................................... 57

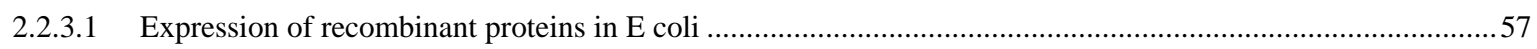

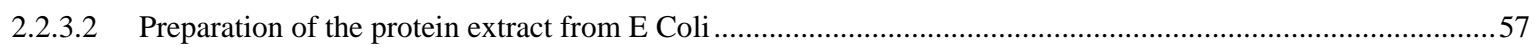

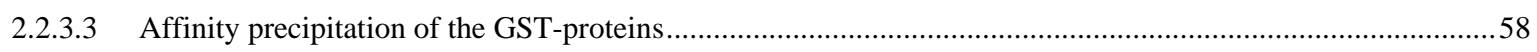

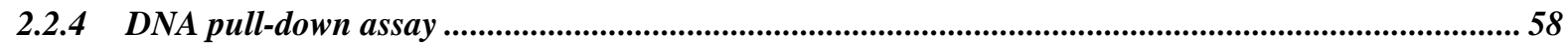

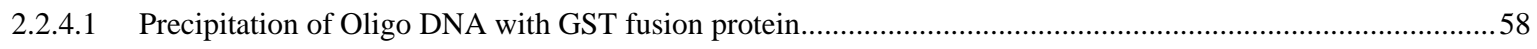

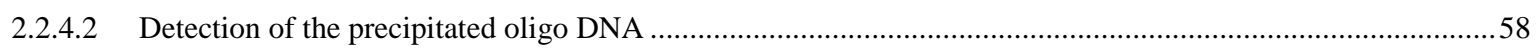

2.2.5 Expression and immunoprecipitation of recombinant Proteins out of Sf9-cells ............................... 59

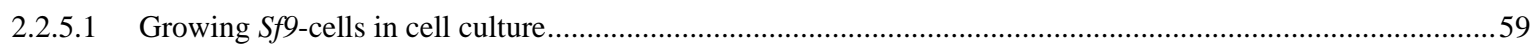

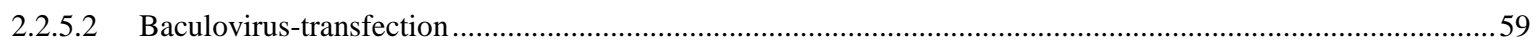

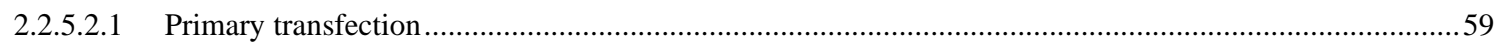

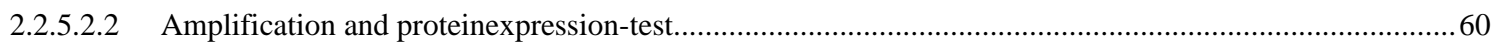

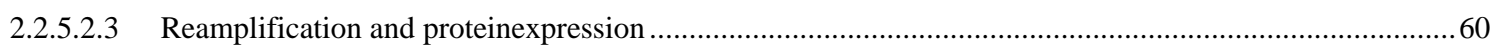

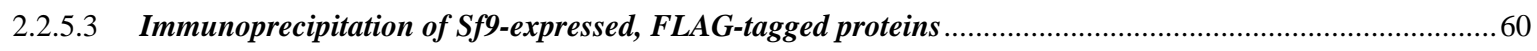

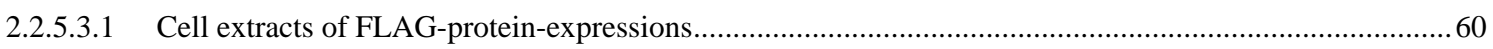

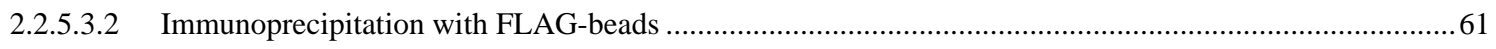

2.2.6 histone methyltransferase (HMT) assay and the detection by fluorography ..................................... 61

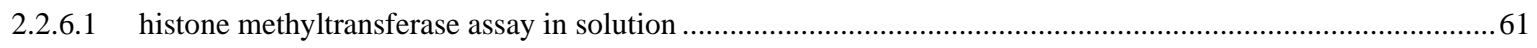

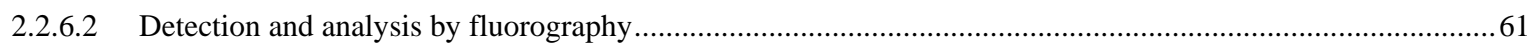

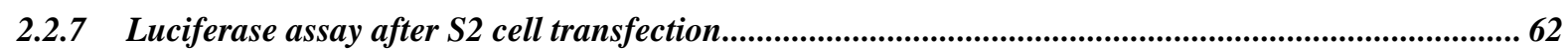

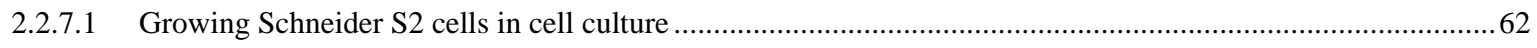

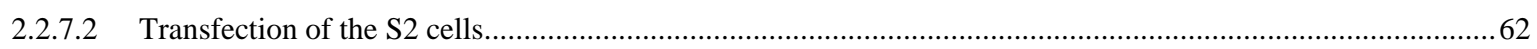

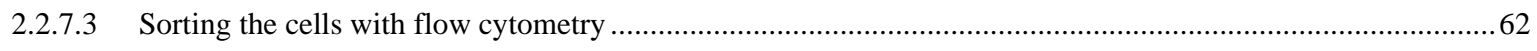

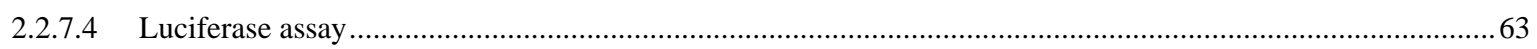

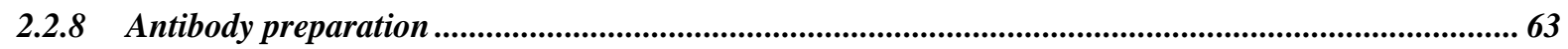




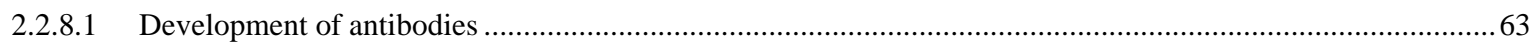

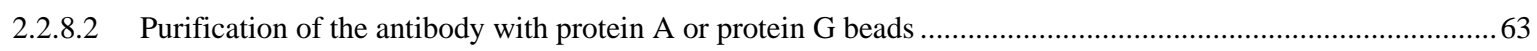

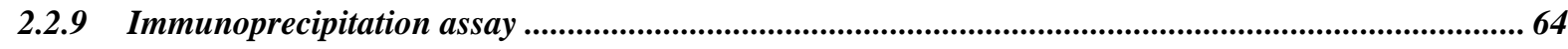

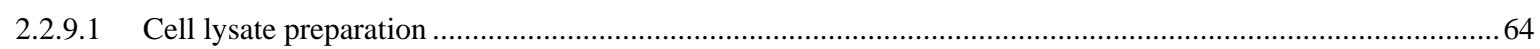

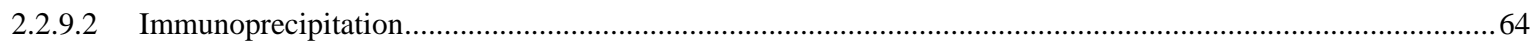

2.2.10 Crosslinked Chromatin Immunoprecipitation (XChIP) ...........................................................6. 65

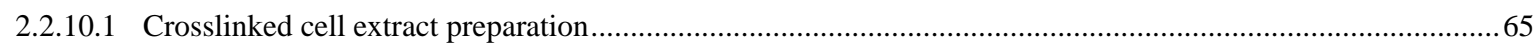

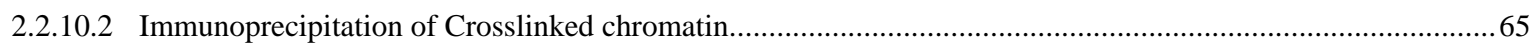

2.2.11 Manipulation of Drosophila melanogaste..................................................................................66

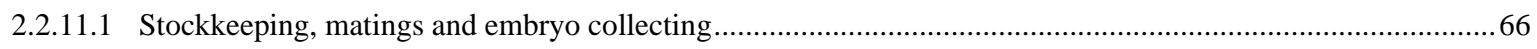

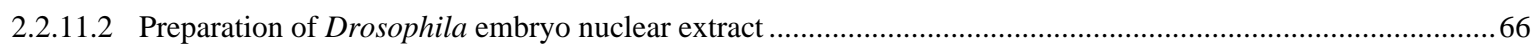

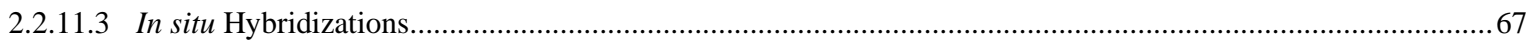

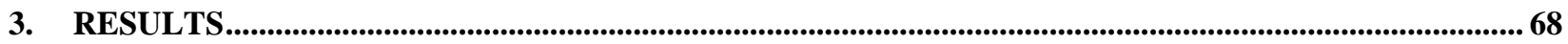

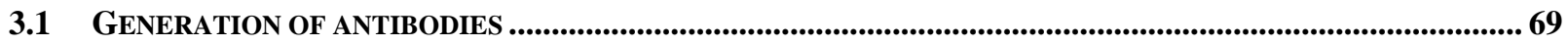

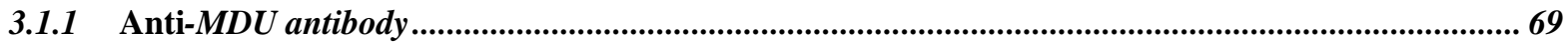

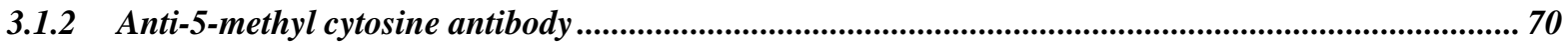

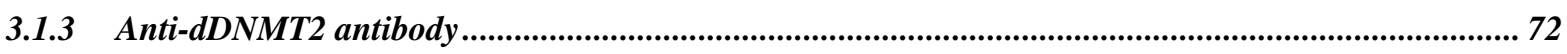

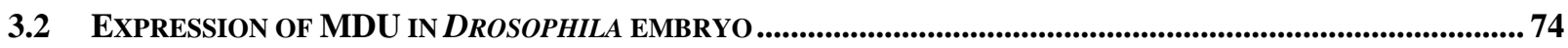

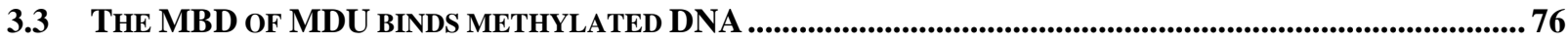

3.4 HISTONE METHYLTRANSFERASE ACTIVITY OF MDU ............................................................................8 81

3.4.1 The SET domain of MDU methylates H3K9 ..............................................................................8 81

3.4.2 MDU tri-methylates lysine 9 in histone H3............................................................................83

3.5 MDU-MEDIATED METHYLATION OF HЗK9 MEDIATES GENE SILENCING.................................................. 85

3.5.1 MDU-mediated repression demonstrated on luciferase assay.............................................................85

3.5.2 MDU-mediated methylation of $\mathrm{H} 3 \mathrm{K9}$ is involved in gene silencing ............................................... 8.

3.6 MDU REGULATES THE EPIGENETIC REPRESSION OF RBF …..............................................................8 89

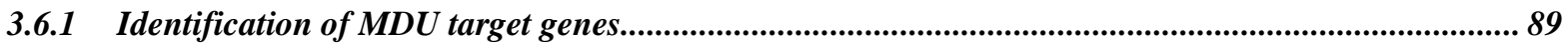

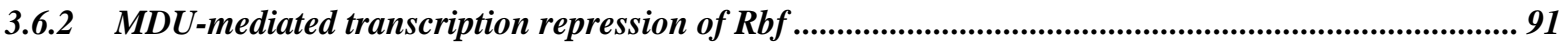

3.6.3 MDU-mediated tri-methylation of H3K9 initiates de novo DNA methylation and silenicng of Rbf .. 93

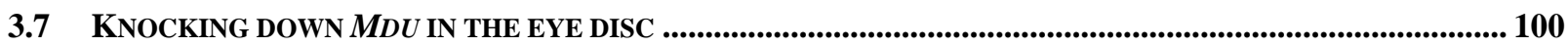

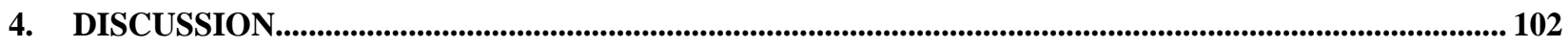

4.1 FUNCTIONAL MDU IS ENCODED BY CG30426 ................................................................................102

4.2 MDU IS A MULTIFUNCTIONAL TRANSCRIPTIONAL REGULATOR ................................................... 104

4.2.1 The MBD of MDU binds methylated CpA motifs ........................................................................... 104

4.2.2 Product specificity of the HMT activity of MDU ............................................................... 108

4.3 INTERPLAY BETWEEN DNA METHYLATION AND H3K9 TRI-METHYLATION; MECHANISM OF MDU MEDIATED RBF SILENCING 111

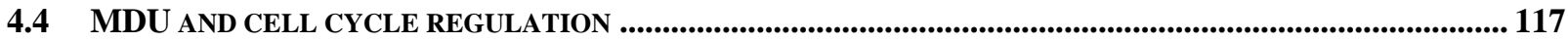




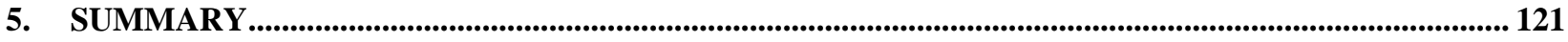

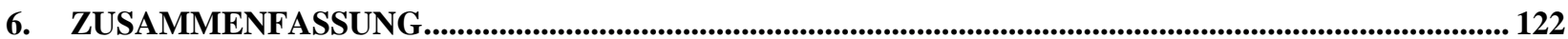

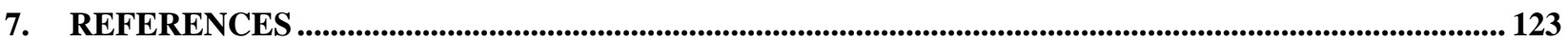

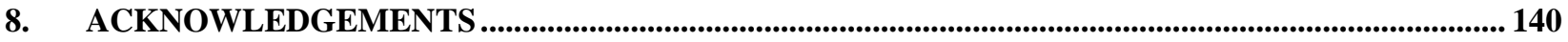

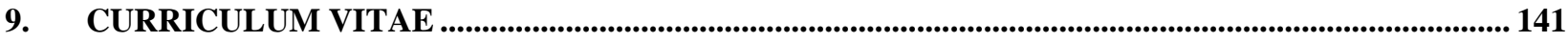




\section{Abbreviations}

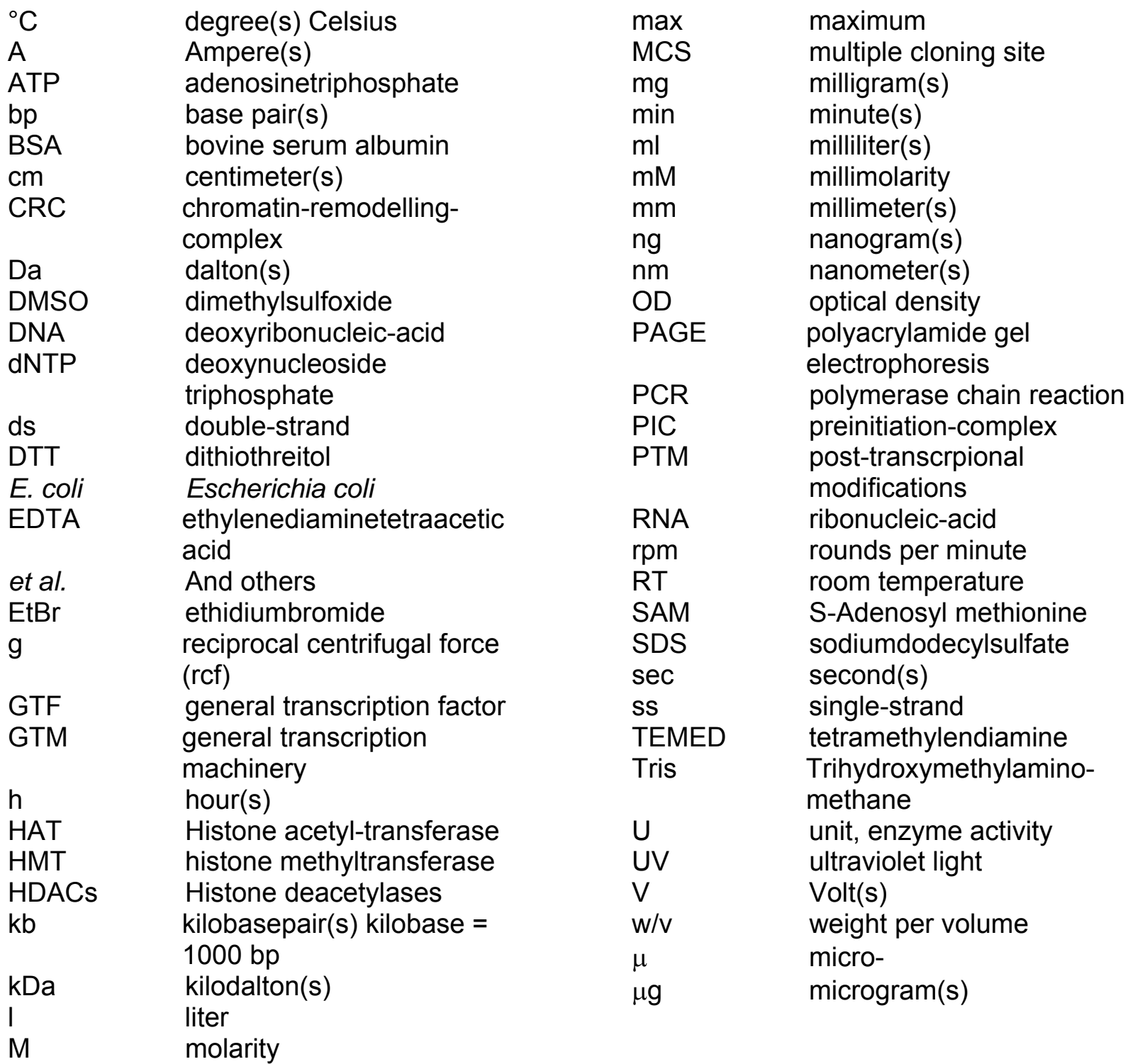




\section{Introduction}

Eukaryotic gene expression is under strict temporal and spatial controls. The regulation of gene expression is made possible by the organization of the genomic DNA into chromatin and the separation of the chromatin from the cytoplasm by the nuclear membrane. Among the several means that can regulate the expression either at the transcriptional or translational level, transcriptional regulation is of major importance. In recent years, people came to recognize one kind of transcriptional regulation that is independent of the alteration in the sequence of the regulated gene and can pass for generations from cell to cell. This level of transcriptional regulation is called epigenetic regulation. Accumulating evidence has shown that this epigenetic regulation of gene expression is associated with the chemical modifications of genomic DNA and/or the DNA binding proteins (mainly histones). Understanding how these modifications are initiated, coordinated, spread and eliminated in various cell processes is intriguing (Fuks 2005; Martin and Zhang 2007).

\subsection{Chromatin}

The chromatin is the complex of genomic DNA and proteins. The chromatin is localized in the nuclei in eukaryotic cells but in the nucleoid in prokaryotic cells (Alberts et al., 2004; Thanbichler et al., 2005). The basic structure of chromatin is the same in all eukaryotes. The DNA of eukaryotic cells is tightly bound to small basic proteins (histones) that package the DNA in an orderly way in the cell nucleus. Besides packing the DNA into a smaller volume to fit in the nucleus, chromatin also has the functions of strengthening the DNA to allow mitosis and meiosis and being regulated by various mechanisms to control expression.

\subsubsection{Structure of chromatin}

In eukaryotic cells, the major chromatin proteins are histones. One pair of each of the 4 core histones, $\mathrm{H} 2 \mathrm{~A}, \mathrm{H} 2 \mathrm{~B}, \mathrm{H} 3$ and $\mathrm{H} 4$, together forms a histone octamer. The fundamental structural unit of eukaryotic chromatin is the nucleosome, which consists of a histone octamer with $147 \mathrm{bp}$ of DNA wrapped 1.75 times around it (Hansen 2002).

The DNA separating nucleosomes is called the linker DNA and can vary in length from 8 
to 114 base pairs. The extranucleosomal linker-DNA that separates core nucleosomes is bound by $\mathrm{H} 1$ histone. $\mathrm{H} 1-\mathrm{H} 1$ interactions participate in the compaction of the 10-nm beads-on-a-string chromatin structure into 30-nm fiber (Thoma et al., 1979). Compared to core-histones, linker H1 histones are evolutionary less conserved (Baxevanis et al., 1995; Lee and Young 2000; Horn and Peterson 2002). The 30-nm fiber can coil to form a hollow tube (100 nm fiber), which finally forms the chromatin (Hansen 2002; Horn and Peterson 2002). This complex compaction and structuring of DNA serves several functions: the overall negative charge of the DNA is neutralized by the positive charge of the histones, and the large DNA fits into the small nucleus.

The final level of packaging is characterized by the $700-\mathrm{nm}$ structure seen in the metaphase chromosome. The condensed piece of chromatin has a characteristic scaffolding structure that can be detected in metaphase chromosomes. This appears to be the result of extensive looping of the DNA in the chromosome (Figure 1).

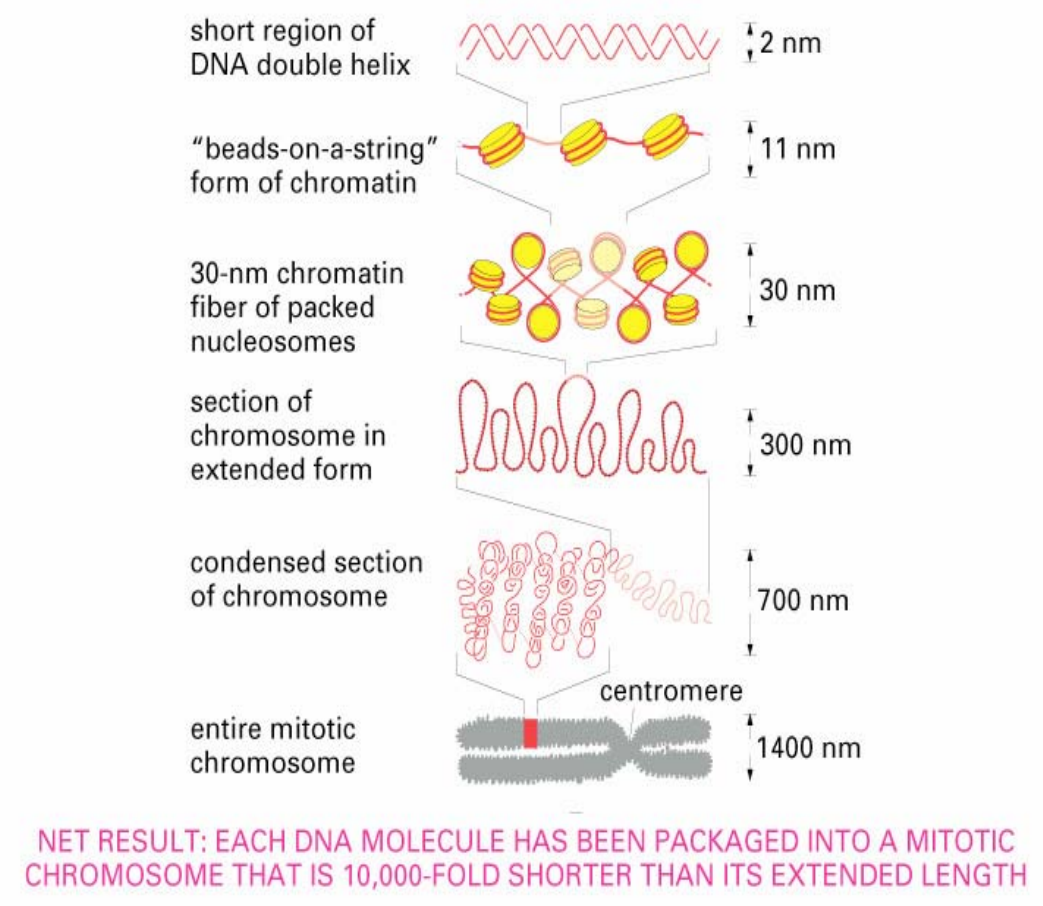

Figure 1. Chromatin packing occurs on several levels. This schematic drawing shows some of the orders of chromatin packing thought to give rise to the highly condensed mitotic chromosome (Alberts et al., 2004).

When chromosomes are stained with dyes such as DAPI or TO-PRO-3, they appear to have alternating light- and dark-stained regions. The light-stained regions are euchromatin and 
contain single-copy, genetically active DNA. The dark-stained regions are heterochromatin and contain repetitive sequences that are genetically inactive (Babu and Verma 1987).

\subsubsection{Regulation of the chromatin activity}

The chromatin structure is not static. The conformation and structure of chromatin change during the alteration of transcription activity and cell cycle progression (Alberts et al., 2004). Recent in vivo data suggest that within highly condensed mitotic chromosomes, the core histones and factors associated with genomic DNA are in dynamic equilibrium, which varies with the phases of mitosis (Chen et al., 2005). Additionally, replication-independent core histone replacement has been demonstrated, and extensive nucleosome displacement and replacement occurs upon gene activation, particularly at transcriptionally active domains of the chromatin (Tagami et al., 2004; Schwabish and Struhl 2004; Schwartz and Ahmad 2005; Thiriet and Hayes 2005). Also, nucleosomes were shown in vitro to have a dynamic equilibrium between a fully wrapped state and a set of partially unwrapped states, in which stretches of DNA transiently detach from the histone surface and then rewrap in a spontaneous and rapid fashion (Li et al., 2005).

\subsection{The epigenetic modifications of DNA and histones}

The word "epigenetics" was created by C. H. Waddington to refer to the model of how genes within a multicellular organism interact with their surroundings to produce a phenotype (Waddington 1957). Arthur Riggs and colleagues later defined epigenetics as "the study of mitotically and/or meiotically heritable changes in gene function that cannot be explained by changes in DNA sequence". Thus, the word "epigenetic" here can be used to describe any aspect other than DNA sequence that influences the development of an organism (Russo et al., 1996). However, this concept tells us what epigenetics is not (inheritance of mutational changes), leaving open what kinds of mechanism are at work.

Today, the definition of epigenetics by A. Bird refers to "the structural adaptation of chromosomal regions so as to register, signal or perpetuate altered activity states" (Bird 2007).

Despite the controversy over how to define epigenetics, it is generally accepted that DNA methylation and the chemical modification of histones play important roles in the epigenetic regulation of gene activity.

DNA methylation refers to the addition of a methyl group to DNA - in multicellular 
eukaryotes to the number 5 carbon of the cytosine pyrimidine ring - to convert it to a 5-methyl cytosine. DNA methylation is catalyzed by DNA methyltransferases (DNMTs). DNA methylation has been extensively investigated in vertebrates and plants, and in the year 2000 it was also found in Drosophila melanogaster (Lyko et al., 2000a; Gowher et al., 2000). DNA methylation is associated with heterochromatin state and inhibition of gene expression in eu- and heterochromatin (Bird and Wolffe 1999).

Core histones play structural roles in chromatin assembly and compaction. Each of the 4 core histones contains the histone fold domain, composed of three $\alpha$-helixes connected by two loops (Kokubo et al., 1994; Arents and Moudrianakis 1995; Baxevanis et al., 1995). This motif is known as the "handshake" motif, and it allows heterodimeric interactions between core histones.

\subsubsection{Covalent modifications of histones}

The $\mathrm{NH}_{2}$-terminal tail of histones, as well as more recently defined positions in the globular domain, can carry post-translational modifications such as acetylation, phosphorylation, ubiquitination, methylation, sumoylation and ADP ribosylation (Ehrenhofer-Murray 2004; Kouzarides 2007; Li et al., 2007). Histone tail modifications can alter DNA-histone and histonehistone interactions within and between nucleosomes and, thus, affect higher-order chromatin structures. Covalent histone modifications collaborate to influence a multitude of cellular processes, including transcription, replication, DNA repair and cell cycle progression (Ehrenhofer-Murray 2004; Kouzarides 2007; Li et al., 2007). A multitude of histone PTMs (Figure 2) has been discovered.

Most of the PTMs are attached to phylogenetically highly conserved amino acids in the histone $\mathrm{NH}_{2}$-terminal tails, for acetylation of lysine $(\mathrm{K})$ residues, methylation of lysine or arginine $(R)$ residues and phosphorylation of serine $(S)$, threonine $(T)$ or tyrosine $(Y)$ residues. Additionally, histones can be ubiquitinated at their COOH-terminus (Zhang and Reinberg 2001; Khorasanizadeh 2004). 
a

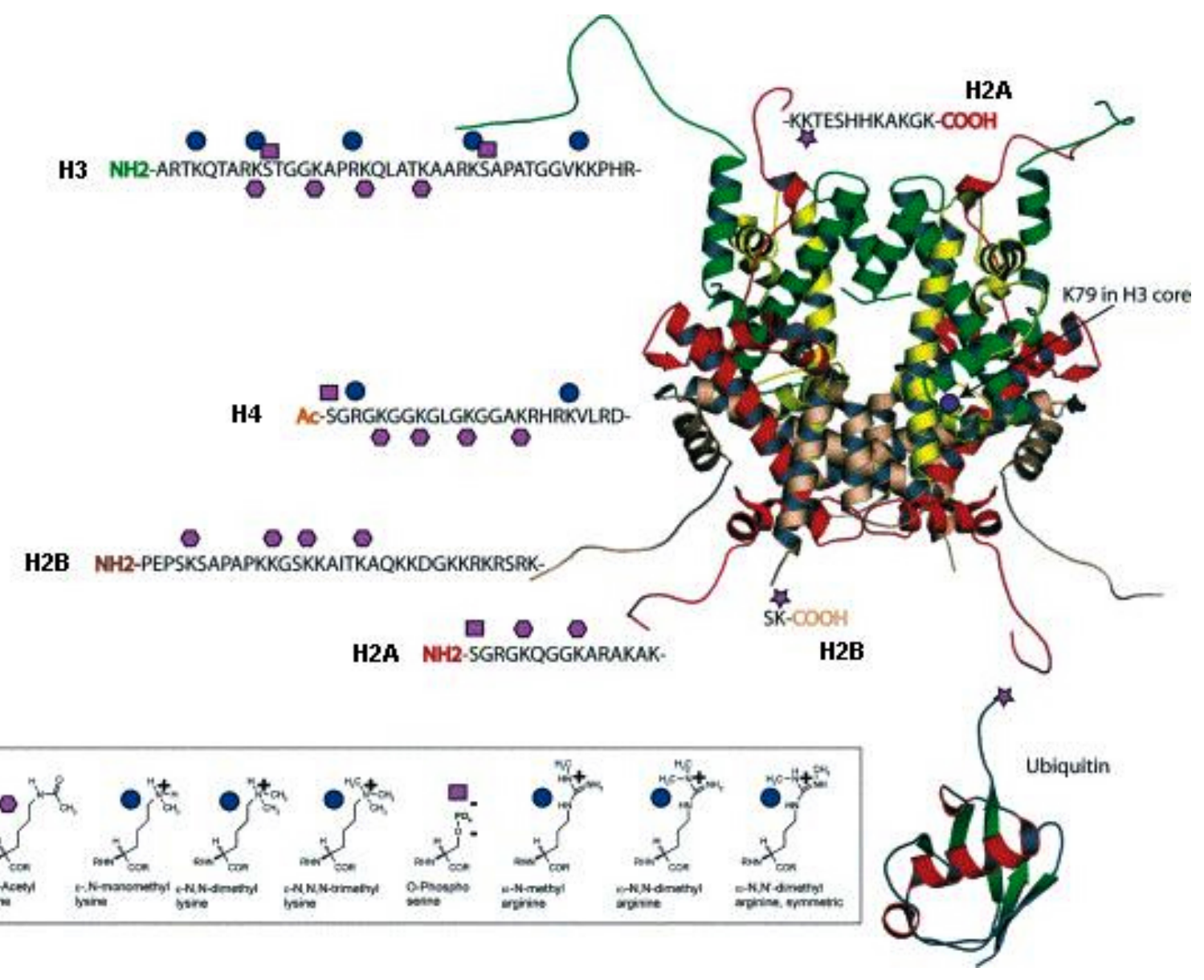

Figure 2. Posttranslational modifications on the core histones (human). a. The histone octamer portion of the nucleosome and the aminosequences of the $\mathrm{NH}_{2}$ - and $\mathrm{COOH}$-terminal ends of single histones are shown; sites of modifications are marked. b. Chemical structure of the covalent modifications of the amino acids shown in "a" (Khorasanizadeh 2004).

Recently, modifications of histones have been discovered in the structured globular domain (Zhang et al., 2003a; Freitas et al., 2004). Several of these modifications occur near the nucleosome lateral DNA binding surface, which indicates that these modifications may affect the interaction of the histone octamer and the DNA (Cosgrove et al., 2004). However, most recent research efforts have focused on post-translational histone modifications occurring at the histone tails and have revealed that histone modifications play an essential role in chromosome function.

PTMs of the core histone tails play important roles in nucleosome stability (BrowerToland et al., 2005), In the context of the dynamic nucleosome, acetylation of histone tails appears to be a prerequisite for nucleosome remodeling by chromatin-remodeling complexes (CRCs) in vivo, thus resulting in disassembly or repositioning of nucleosomes (Lomvardas and 
Thanos 2001; Reinke and Horz 2003; Nourani et al., 2004). PTMs of histones may also help define the condensed state of the chromatin fiber and higher order structures by facilitating nucleosome assembly or disassembly (Ito 2007). They have been implicated in transcriptional activation, silencing, and DNA replication (Zhou et al., 2005; Shogren-Knaak and Peterson 2006), too.

The correlation of specific histone modifications with the execution of specific biological events gave rise to the histone code hypothesis, which postulates that specific histone modifications determine chromosome function (Strahl and Allis 2000). Specifically modified amino acid residues are also referred to as "marks", bearing information for the specific functional code of the packaged DNA (Strahl and Allis 2000).

\subsubsection{Histone methylation}

Methylation occurs on lysine and arginine residues in histones (Walsh 2005). Arginine can be methylated in three ways on the guanidino group: monomethylated (MMA), symmetrically dimethylated (sDMA) and asymmetrically dimethylated (aDMA), each of which has potentially different functional consequences. The methylation of arginine residues is catalyzed by the protein arginine N-methyltransferase (PRMT) family of enzymes (Bedford and Richard 2005). Recent research suggested that the Jumonji-domain-containing-6-protein (JMJD6) demethylates histone $\mathrm{H} 3$ at arginine 2 (H3R2) and histone H4 at arginine 3 (H4R3) (Chang et al., 2007). Arginine methylation is involved in a number of different cellular processes, including transcriptional regulation, RNA metabolism and DNA damage repair (Bedford and Richard 2005).

The $\varepsilon$-aminogroup of lysine residues can be mono-, di- or tri-methylated (Walsh 2005). The enzymes that can add methyl groups to the lysine residue are called histone methyltransferases (HMTs). The functional domain of HMTs containing the enzymatic activity responsible for lysine methylation of histone tails is called SET domain, which consists of 130 amino acids folding into three discrete $\beta$-sheet regions flanked by $\alpha$-helices (Khorasanizadeh 2004). The cofactor S-adenosyl-L-methionine (SAM) binds to a concave surface of the enzyme, providing methyl groups for modification. The histone tail inserts as a parallel strand between two strands of the SET domain (Zhang and Reinberg 2001; Khorasanizadeh 2004). A HMT that is atypical because it does not contain a SET domain is Dot1, which, despite the lack of SET domain, still specifically methylates lysine 79 of histone $\mathrm{H} 3$ in the core domain. Dot1 methylation 
of lysine 79 in $\mathrm{H} 3$ mediates gene-silencing mechanisms in yeast (Park et al., 2002; Min et al., 2003).

Recently, histone lysine methylation was shown to be reversible, and this function is carried out by two families of enzymes: amine oxidases such as LSD1 and hydroxylases of the JmjC family (Shi et al., 2004; Schneider \& Shilatifard 2006; Klose \& Zhang 2007). Because LSD1 requires a protonatable methyl ammonium group, only mono- and di-methyl forms can be substrates. In contrast, JmjC family members can also demethylate trimethylated lysine (Klose \& Zhang 2007; Shi 2007). LSD1 can demethylate lysine 4 or lysine 9 of H3, depending on its associated proteins (Klose \& Zhang 2007; Shi 2007). Many JmjC family members have unique substrate specificities, with demethylases for lysine 4, 9, 27 or 36 of histone 3 being recently characterized (Shi 2007).

Histone lysine methylation has been linked to both transcriptional activation and repression (Roth et al., 2001; Zhang and Reinberg 2001). For example, the lysine 4 methylation on histone $\mathrm{H} 3$ is catalyzed by COMPASS, which is mostly associated with the early elongating form of RNA polymerase II at actively transcribed genes (Shilatifard 2006). H3K36 methylation by Set2 is associated with the transcriptional elongation form of RNA polymerase II (Shilatifard 2006). On the contrary, histone H3K9 methylation is linked to gene silencing. The Suv39 protein methylates histone $\mathrm{H} 3 \mathrm{~K} 9$ and localizes to transcriptionally silent heterochromatin, where it recruits the transcriptional repressor HP1 (Richards \& Elgin 2002; Ayyanathan et al., 2003; Grewal \& Moazed 2003; Sims et al., 2003). However, the molecular mechanism of chromatin compaction by HP1 remains unclear. Suv39 and HP1 have also been implicated in transcriptional repression at euchromatic loci, because the co-repressor protein $\mathrm{Rb}$ (retinoblastoma) recruits Suv39 and HP1 to cell-cycle control genes including cyclinE (Nielsen et al., 2001; Vandel et al., 2001). Like histone H3K9 methylation, methylation of histones H3K27 and H4K20 is also involved in heterochromatin formation and heterochromatic gene silencing (Shilatifard 2006).

\subsubsection{Histone acetylation}

The acetylation and deacetylation on histone $\mathrm{NH}_{2}$-terminal tail lysine residues are catalyzed by histone acetyltransferase (HAT) and histone deacetylase (HDAC), respectively, and the reversible histone acetylation is involved in transcriptional gene regulation (Grunstein 1997; Jenuwein and Allis 2001; Berger 2002; Kurdistani and Grunstein 2003). 
HATs are present in transcription factors and CRCs (Roth et al., 2001). HAT domains contain a central conserved core unit that is important for acetyl-coenzyme-A (acetyl-CoA) binding and a cleft, used for substrate recognition that lies directly over the cofactor-binding pocket (Khorasanizadeh 2004). HATs reside within the context of large multisubunit complexes and are separated into two main classes of HATs: Type A (nuclear) and type B (cytoplasmic) (Narlikar et al., 2002). Nuclear regulatory complexes contain mainly type A HATs, of which three families have been identified: the GNAT family (containing yeast GCN5 and human PCAF), the MYST family (containing yeast complex NuA4 with its HAT yESA1) and the P300/CBP family.

HDACs fall into three main classes (Khochbin et al., 2001; Marmorstein 2001) that reside in different complexes. The class I HDAC family contains, for example, the remodeling-complex NuRD with its subunit HDAC1 or the transcriptional corepressor Sin3 with its subunit HDAC2. Class II HDACs have yet to be purified. A prominent member of the class III HDACs is Sir2, involved in heterochromatin silencing at silent mating loci, telomeres and ribosomal DNA (Moazed 2001). This interplay between HAT and HDAC complexes maintains the steady-state level of acetylation (Reid et al., 2000; Vogelauer et al., 2000).

Hyperacetylation of lysine residues in the histone tails was proposed to be involved in the activation of transcription over 40 years ago (Allfrey et al., 1964). According to the charge neutralization model, acetylation brings in a negative charge that neutralizes the positive charge on the histones and decreases the interaction of the $\mathrm{NH}_{2}$-termini of histones with the negatively charged phosphate groups of DNA. As a consequence, the condensed chromatin is transformed into a more relaxed structure, which supports gene transcription (Davie and Chadee 1998).

More recently, the finding that acetyl lysine residues on histone tails form binding sites for bromo-domains on various proteins suggests a quite different function (Dyson et al., 2001; Zeng and Zhou 2002; Loyola and Almouzni 2004; Yang 2004). This finding indicates that acetylation, akin to many protein phosphorylation events, creates a new binding surface to recruit other proteins to the nucleosome.

The latest study suggests that dynamic turnover rather than stably enhanced histone acetylation levels may be relevant to transcription. This hypothesis challenges the charge neutralization model by revealing the existence of a small subset of nucleosomes and histones that are continuously subject to the action of HATs and HDACs. This hypothesis requires that the HATs and HDACs act continuously on the affected histone tail (Hazzalin and Mahadevan 2005). Methylation at a specific lysine residue (K4) is involved in targeting histone tails for continuous acetylation and deacetylation (Clayton et al., 2006). 


\subsubsection{Histone phosphorylation}

Histones are phosphorylated at various amino acids (Bradbury 1992; Koshland and Strunnikov 1996; Barber et al., 2004). Several distinct kinases are involved in the phosphorylation of histones on different residues. Phosphorylation of histone $\mathrm{H} 2 \mathrm{~A}$ is dependent on phosphatidylinositol-3-OH kinases such as Mec1 in yeast (Foster and Downs 2005). Histone $\mathrm{H} 2 \mathrm{~B}$ phosphorylation is catalyzed by the sterile-20 kinase in yeast and Mst1 (mammalian sterile20-like kinase) in mammals (Ahn et al., 2005). Histone H3S10 and H3S28 phosphorylation during mitosis is regulated by the Aurora kinases, which are highly conserved from yeast to humans (Nowak \& Corces 2004). Recently, phosphorylation of serine residue 1 in H4 (H4S1) was linked to sporulation in yeast and spermatogenesis of $D$. melanogaster and mice. In yeast, a sporulation-specific kinase, Sps1, is required for the H4S1 phosphorylation (Krishnamoorthy et al., 2006; Wendt \& Shilatifard 2006).

Histone phosphorylation is involved in the cell-cycle regulation. For example, histone H4S1 phosphorylation has an evolutionarily conserved role in chromatin compaction during the later stages of gametogenesis (Krishnamoorthy et al., 2006). Histone H2A phosphorylation is also associated with mitotic chromosome condensation (Barber et al., 2004). The phosphorylation of histone $\mathrm{H} 2 \mathrm{~B}$ is linked to the apoptotic chromatin condensation in yeast and human cells (Ahn et al., 2005).

Histone phosphorylation can also have a role in transcription. The phosphorylation of histone $\mathrm{H} 3 \mathrm{~S} 10$ has been shown to establish the transcriptional competence of early response genes such as FOS and JUN (Nowak \& Corces 2004). Drosophila TFIID subunit TAF1 was shown to phosphorylate serine residue 33 in H2B (H2BS33), and H2BS33 phosphorylation is essential for transcriptional activation events that promote cell cycle progression and development (Maile et al., 2004).

\subsubsection{Histone ubiquitination}

Ubiquitin (Ub) is a 76-amino acid protein that is ubiquitously distributed and highly conserved throughout eukaryotic organisms. A variety of cellular processes including protein degradation, stress response, cell-cycle regulation, protein trafficking, endocytosis signaling, and transcriptional regulation have been linked to this molecule (Pickart 2001). Ub is covalently attached to a target protein through an isopeptide bond between its $\mathrm{COOH}$-terminal glycine and 
the $\varepsilon$-amino group of a lysine residue on the acceptor protein. Attachment of an Ub molecule to the side chain of a lysine residue in the acceptor protein is a complex process involving multienzyme-catalyzed steps, including E1-activating, E2-conjugating and E3 ligase enzymes (Pickart 2001). Removing the ubiquitin moiety, however, is achieved through the action of enzymes called isopeptidases (Wilkinson 2000).

Histones can also be modified through ubiquitination (Jason et al., 2002). Histone $\mathrm{H} 2 \mathrm{~A}$ was the first protein identified to be ubiquitinated (Goldknopf et al., 1975). H2B is ubiquitinated as well (West and Bonner 1980). Although uH2B is less abundant $(1 \%-2 \%)$ than $\mathrm{uH} 2 \mathrm{~A}(5 \%-$ $15 \%$ ), it appears to be widely distributed throughout eukaryotic organisms from budding yeast to humans. Like $\mathrm{H} 2 \mathrm{~A}$, the ubiquitinated site of $\mathrm{H} 2 \mathrm{~B}$ has been mapped to $\mathrm{COOH}$-terminus lysine residues, namely, Lysine 120 in human $\mathrm{H} 2 \mathrm{~B}$ and Lysine 123 in yeast H2B (Thorne et al., 1987). In addition to $\mathrm{H} 2 \mathrm{~A}$ and $\mathrm{H} 2 \mathrm{~B}, \mathrm{H} 3$ and $\mathrm{H} 1$ ubiquitination has been reported (Chen et al., 1998; Pham and Sauer 2000).

Specific E2s and E3s are required for ubiquitination of histones. In budding yeast, Rad6, an E2-conjugating enzyme, in conjunction with Bre1, an E3-ligase, is required for histone $\mathrm{H} 2 \mathrm{~B}$ monoubiquitination, whereas for histone $\mathrm{H} 2 \mathrm{~A}$, the polycomb group RING finger protein Ring1b acts as the E3-ligase (Zhang 2003c; Fang et al., 2004; Shilatifard 2006).

Histone ubiquitination is important in the regulation of gene expression. Both positive and negative effects of histone ubiquitination on transcription have been reported. For example, nucleosomes of transcriptionally poised hsp 70 genes contain up to $50 \% \mathrm{uH} 2 \mathrm{~A}$, whereas nucleosomes of untranscribed satellite DNA contain only one uH2A per 25 nucleosomes (Levinger and Varshavsky 1982). Furthermore, both $\mathrm{uH} 2 \mathrm{~A}$ and $\mathrm{uH} 2 \mathrm{~B}$ are enriched around transcriptionally active sequences in bovine thymus, chicken erythrocytes, and Tetrahymena macronuclei (Nickel et al., 1989). However, different results have been reported. For example, the active immunoglobulin $\kappa$-chain gene is packaged with nonubiquitinated histones (Huang et al., 1986). Random distribution of $\mathrm{uH} 2 \mathrm{~A}$ in chromatin fractions was reported in a study involving DNase I sensitivity to differentiate active and inactive transcription regions (Dawson et al., 1991). In addition, ubiquitinated histones have been found in transcriptionally inactive compartments, such as the Tetrahymena micronuclei (Nickel et al., 1989) or the sex body of mouse spermatids (Baarends et al., 1999). Accumulating evidence suggests that links between transcriptional status and histone ubiquitination are context dependent, based on gene location or possibly the presence of other histone covalent modifications, especially histone methylation (Zhang 2003c). 


\subsubsection{SET domain proteins}

The SET domain was first recognized as a conserved sequence in three D. melanogaster proteins: a modifier of position-effect variegation, suppressor of variegation 3-9 (Su(var)3-9) (Tschiersch et al., 1994), the polycomb-group chromatin regulator enhancer of zeste $(E(z))$ (Jones and Gelbart 1993), and the trithorax-group chromatin regulator trithorax (Trx) (Stassen et al., 1995). The SET domain is the landmark motif of lysine-specific HMTs. The function of SETdomain proteins is to transfer a methyl group from S-adenosyl-L-methionine (AdoMet) to the amino group of a lysine residue of histones or other protein, leaving a methylated lysine residue (Dillon et al., 2005).

SET domain proteins can be divided into several classes according to their structure and surrounding motifs -- the SUV39, SET1, SET2, EZ, RIZ, SMYD, and SUV4-20 families -- as well as a few orphan members such as SET7/9 and SET8 (also called PR-SET7). Proteins within each family have similar sequence motifs surrounding the SET domain, and they often share a high level of similarity in the SET domain (Dillon et al., 2005).

Currently known structures of SET-domain proteins show that the SET domain forms a novel $\beta$-fold not seen in other previously characterized AdoMet-dependent methyltransferases (Schubert et al., 2003). The fold has a series of curved $\beta$-strands forming several small sheets flanked by pre-SET (or N-SET) and post-SET (or C-SET) domains or regions. A "pseudoknot" structure surrounded by the $\beta$-sheets is seen in the SET domain because the carboxyl terminus runs through an opening of a short loop formed by a preceding stretch of the sequence. This remarkable pseudoknot fold brings together the two most-conserved sequence motifs of the SET domain, H(R)FFNHSC (or NHSC) and ELXFDY, to form an active site in a location immediately next to the pocket where the methyl donor binds and to the peptide-binding cleft (Figure 3 ). 

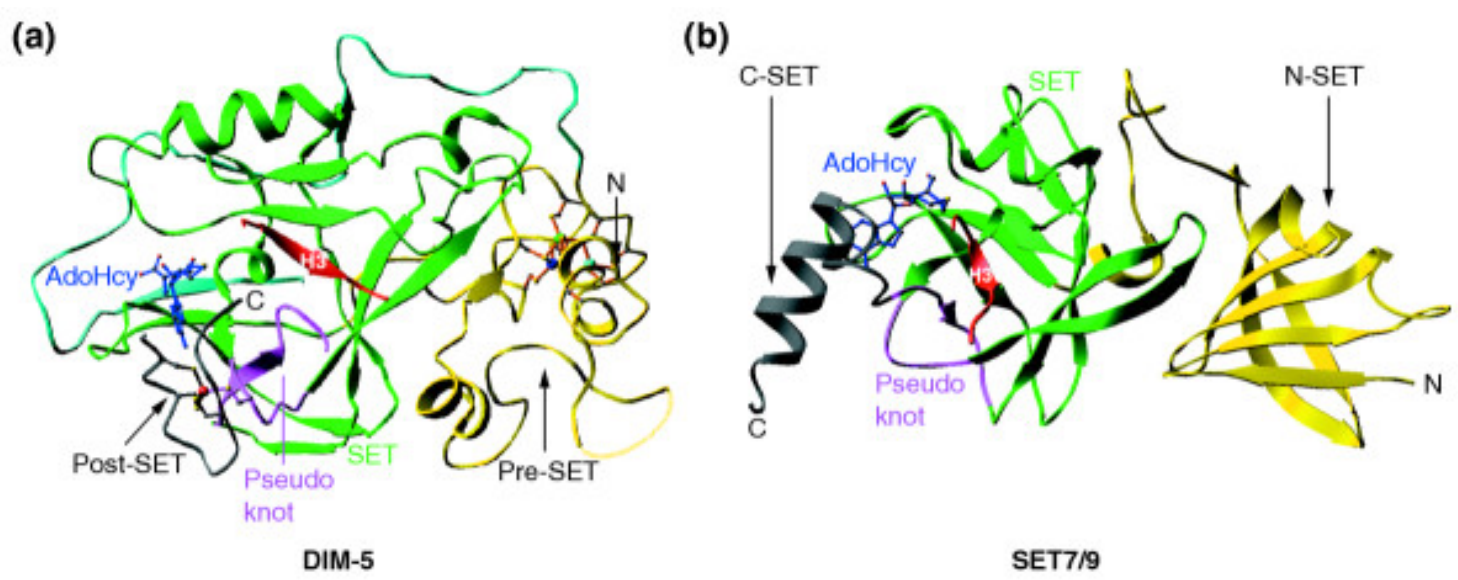

Figure 3. Representative examples of SET-domain-containing structures. a. The pre-SET, SET, and post-SET domains in DIM-5 of Neurospora crassa. b. N-SET, SET, and C-SET domains in SET7/9. The pseudoknot formed by two conserved SET motifs and the bound histone $\mathrm{H} 3$ peptide are illustrated. The reaction byproduct AdoHcy is in stick representation, and the zinc ions are shown as balls (Dillon et al., 2005).

The $\mathrm{NH}_{2}$-terminal flanking region of the SET domains exhibits structural variability. In DIM-5, this region is composed of a nine cysteine-three $\mathrm{Zn}^{2+}$ cluster, classified as a PreSET domain (Zhang et al., 2003b), whereas in SET7/9 (Xiao et al., 2003b) and SET8 (Xiao et al., 2005), the nSET regions are composed of a $\beta$-sheet domain and an $\alpha$-helix, respectively. The cSET region plays important roles in substrate binding and catalysis in SET-domain enzymes. This region is often disordered in the apoenzyme, but folds cooperatively upon substrate binding, forming the S-adenosylmethionine- and lysine-binding pockets. In DIM-5, the cSET region forms a single 4-cysteine- $\mathrm{Zn}^{2+}$ cluster, known as a PostSET domain (Zhang et al., 2003b), whereas in SET7/9 (Xiao et al., 2003b) and SET8 (Xiao et al., 2005), the C-termini adopt helical conformations.

The SUV39 protein was the first HMT to be identified (Rea et al., 2000). Members of this family include human SUV39H1, murine Suv39h2, Schizosaccharomyces pombe Cryptic loci regulator 4 (CLR4) and D. melanogaster Su(var)3-9. These proteins specifically methylate lysine 9 of histone H3 (H3K9). SETDB1 and its Drosophila ortholog MDU (CG30426) are also from this family.

In general, HMTs possess narrow substrate specificities, frequently targeting a single lysine residue within their respective substrates. SUV39 targets to H3K9, SET8 and DIM-5 show remarkable specificity for histone H4 Lys20 (Nishioka et al., 2002a, Fang et al., 2002) and 
histone H3 Lys9 (Tamaru and Selker 2001), and SET1 of S. cerevisiae di- or tri-methylates H3K4 (Santos-Rosa et al., 2002). The strict lysine specificity of these enzymes is in distinct contrast to Drosophila ASH1 (which targets H3K4, H3K9 and H4K20) (Beisel et al., 2002), mammalian G9a, human EZH1 and EZH2 and mouse NSD1, enzymes that can methylate two or more different lysine residues. In some cases, the functions of SET-domain enzymes are not confined to histone methylation. For instance, human SET7/9 possesses a broader substrate specificity and can methylate several targets, including Lysine 4 of histone H3 (Wang et al., 2001; Nishioka et al., 2002b), Lysine 372 of p53 (Chuikov et al., 2004) and Lysine 189 of TAF10 (Kouskouti et al., 2004).

HMTs are involved in the cross talk between DNA and histone methylation. DIM-5 trimethylates H3K9 (Zhang et al., 2003b), and this marks chromatin regions for DNA methylation (Tamaru et al., 2003). Other members of the SUV39 family -- KRYPTONITE of $A$. thaliana (Jackson et al., 2002; Jackson et al., 2004), Suv39h1 of mouse (Lehnertz et al., 2003), and mammalian G9a (Xin et al., 2003) -- have been implicated in DNA methylation. Di-methylation of H3K9 is the critical mark for inducing DNA methylation by KRYPTONITE (Jackson et al., 2004).

\subsubsection{DNA methylation}

DNA methylation is found in the genomes of prokaryotes and eukaryotes. In prokaryotes, DNA methylation occurs on both cytosine and adenine bases and encompasses part of the host defense restriction system (Wilson and Murray 1991). In multicellular eukaryotes, however, methylation is confined to cytosine bases and is linked to silencing of gene expression. The DNA methylation patterns differ from species to species (Adams 1996). DNA methyltransferases are required to catalyze methylation of DNA, and proteins with affinity to 5-methyl cytosine can specifically recognize this epigenetic signal (Bird 2002).

\subsubsection{Patterns of DNA methylation}

DNA methylation varies in different organisms. The percentage of methylated cytosines ranges from $0.1-3 \%$ in the genomic DNA of insects, $5 \%$ in mammals and birds, and $10 \%$ in fish and amphibians, to more than $30 \%$ in some plants (Adams 1996). At the low extreme is the nematode worm Caenorhabditis elegans, whose genome lacks detectable 5-methyl cytosine and does not encode a conventional DNA methyltransferase (Adams 1996). Another invertebrate, the 
insect $D$. melanogaster, long thought to be devoid of methylation, has a DNA methyltransferaselike gene dDNMT2 (Hung et al., 1999; Tweedie et al., 1999) and is reported to contain very low 5-methyl cytosine levels (Lyko et al., 2000a; Gowher et al., 2000). DNA methylation in Drosophila occurs predominantly at CpT/A motif rather than $\mathrm{CpG}$ motif, which is the major target for methylation in animals (Bird 1980). Most other invertebrate genomes have moderately high levels of methyl-CpG concentrated in large domains of methylated DNA separated by equivalent domains of unmethylated DNA (Bird et al., 1979; Tweedie et al., 1997). At the opposite extreme of $C$. elegans are the vertebrates, which have the highest levels of 5-methyl cytosine found in the animal kingdom (Adams 1996). Vertebrate methylation is dispersed over much of the genome, a pattern referred to as global methylation. The variety of DNA methylation patterns in animals raises the possibility that different DNA methylation patterns reflect different functions of the DNA methylation system (Colot and Rossignol 1999).

The most striking feature of vertebrate DNA methylation patterns is the presence of CpG islands. $\mathrm{CpG}$ islands are regions of DNA with a high $\mathrm{G}+\mathrm{C}$ content and a high frequency of $\mathrm{CpG}$ dinucleotides relative to the bulk genome. CpG dinucleotides appear in the $\mathrm{CpG}$ islands at a frequency of approximately every 10 base pairs. By contrast, the methylated majority of the genome is relatively AT-rich and has methyl-CpGs approximately every 100 base pairs. The low percentage of $\mathrm{CpGs}$ in bulk genomic DNA is due to the mutability of 5-methyl cytosine, which tends to mutate to T (Bird 1980; Duncan and Miller 1980).

$\mathrm{CpG}$ islands are generally unmethylated and typically occur at or near the transcription start site of genes, particularly housekeeping genes (Bird 1980; Bird 2002). Computational analysis sequence predicts 29,000 CpG islands in the human genome (Lander et al., 2001; Venter et al., 2001). Earlier studies estimated that $\sim 60 \%$ of human genes are associated with $\mathrm{CpG}$ islands, of which most are unmethylated at all stages of development and in all tissue types to prevent the genes from being silenced (Antequera et al., 1990). A small but significant proportion of all $\mathrm{CpG}$ islands become methylated during development, and when this happens the associated promoter is stably silent. Developmentally programmed CpG island methylation of this kind is involved in genomic imprinting and $X$ chromosome inactivation (Bird 2002).

The de novo methylation events occur in germ cells or the early embryo (Jaenisch et al., 1982), which suggests that de novo methylation is particularly active at these stages. However, some evidence exists for de novo methylation occurring in adult somatic cells. A significant proportion of all human $\mathrm{CpG}$ islands are prone to progressive methylation in certain tissues during aging (Issa 2000), or in abnormal cells such as cancers cells (Baylin and Herman 2000) and permanent cell lines (Harris 1982; Antequera et al., 1990; Jones et al., 1990). 
Although cytosine methylation in animals is prevalent in symmetrical CpG dinucleotides, in plants, it is often found in symmetrical $\mathrm{CpG}$ and $\mathrm{CpXpG}$ contexts as well as in nonsymmetrical CpXpX (X = C, A, T) (Gruenbaum et al., 1981; Meyer et al., 1994).

DNA methylation is involved in transcriptional regulation, mainly the reinforcing and stabilizing of the transcriptionally silent state of genes (Bird 2002). Phenomena that deserve special interest are repression of the transposable element and genomic imprinting. In plants, DNA methylation is also associated with response to pathogen infection (Pavet et al., 2006) and transgene silencing (Matzke et al., 1989).

Two modes of how DNA methylation silences gene expression can be envisaged. The first mode involves direct interference of the methyl group in binding of a protein to its cognate DNA sequence. Many factors are known to bind CpG-containing sequences, and some of these fail to bind when the $\mathrm{CpG}$ is methylated. Strong evidence for involvement of this mechanism in gene regulation comes from studies of the role of the CTCF protein in imprinting at the H19/lgf2 locus in mice (Bell and Felsenfeld 2000; Hark et al., 2000; Szabo et al., 2000; Holmgren et al., 2001).

The second mode of repression is opposite to the first, because it involves proteins that are attracted to, rather than repelled by, methylated DNA. Characterization of a family of methylCpG binding proteins showed that each contains a region closely related to the methyl-CpG binding domain (MBD) of MeCP2 (Nan et al., 1993; Nan et al. 1997; Cross et al., 1997; Hendrich and Bird 1998). Four of these proteins-MBD1, MBD2, MBD3, and MeCP2-have been implicated in methylation-dependent repression of transcription (Bird and Wolffe 1999). An unrelated protein, Kaiso, has also recently been shown to bind methylated DNA and bring about methylation-dependent repression in model systems (Prokhortchouk et al., 2001).

\subsubsection{The DNA methyl transferases}

In mammals DNA methylation patterns are established and maintained by at least five DNA methyltransferase: DNMT1, DNMT2, DNMT3a, DNMT3b and DNMT3L (Bestor 2000; Li 2002). The enzymes contribute to different steps of DNA methylation processes, but as more studies reveal, a considerable level of cooperation and functional overlap exists among them. The classical maintenance methyltransferase is known as DNMT1 (Bestor et al., 1988; Yen et al., 1992). This enzyme is responsible for maintenance of methylation pattern during DNA replication. DNMT1 is the most abundant DNA methyltransferase in mammalian cells (Robertson et al., 1999). It predominately methylates hemimethylated CG di-nucleotides in the mammalian 
genome. DNMT1 consists of the regulatory domain at the $\mathrm{NH}_{2}$-terminus. The $\mathrm{COOH}$-terminus constitutes the catalytic domain. The domains are joined by Gly-Lys repeats (Robertson et al., 1999).

The mammalian genome encodes two cytosine methyltransferases of the DNMT3 family, DNMT3a and DNMT3b. Both enzymes are generally regarded as de novo DNA methyltransferases, although they may also play a role in the maintenance of methylation (Liang et al., 2002; Hsieh 2005). DNMT3a and DNMT3b are highly expressed during early development stages, and most of the de novo methylation occurs at that time (Okano et al., 1998; Okano et al., 1999). Both enzymes are necessary for proper development of mammalian embryos by their establishing new methylation patterns, and both are required, especially DNMT3b, for methylation of specific genomic regions such as pericentromeric repetitive sequences and CpG islands on the inactive $X$ chromosome (Okano et al., 1999; Hansen 2003).

Enzymes from the DNMT3 family show no preference for hemimethylated over fully unmethylated DNA substrates. Additionally, DNMT3a shows methylation of non-CpG sites both in vitro and in vivo (Okano et al., 1998; Ramsahoye et al., 2000). These biochemical features demonstrate the de novo methylation function of the DNMT3 family.

The DNMT3 family was recently suggested to contain one more member - a third homolog called DNMT3L (DNA methyltransferase 3-like), which is expressed specifically in germ cells (Aapola et al., 2000). DNMT3L has no detectable methyltransferase activity but is essential for establishment of a subset of methylation patterns in both male and female germ cells by functioning as a regulatory factor (Bourc'his et al., 2001).

DNMT2 is another cytosine methyltransferase family protein. In Drosophila, it is called dDNMT2. It is the most widely distributed, and its orthologs are present even in species that are believed not to methylate DNA (i.e., Schizosaccharomyces pombe, C. elegans). The conservation of all 10 DNMT-specific catalytic motifs suggests that DNMT2 should be a DNA cytosine methyltransferase. Initial studies failed to detect an enzymatic activity for DNMT2, but more recent reports have provided evidence for a low but significant DNA methyltransferase activity for humans (Hermann et al., 2003), Drosophila (Kunert et al., 2003), Entamoeba (Fisher et al., 2004) and Dictyostelium (Kuhlmann et al., 2005) DNMT2. However, DNMT2 from various organisms methylates cytosine 38 in the anticodon loop of tRNAAsp (Goll et al., 2006).

DNMT2 expression is elevated during early developmental stages in Drosophila and in zebrafish (Hung et al., 1999; Lyko et al., 2000b; Kunert et al., 2003), which suggests a role for DNMT2 in development. Although DNMT2 mutant mice, flies and plants (Arabidopsis thaliana) are viable and fertile, DNMT2 mutant zebrafishes show defective liver, brain and retina 
development (Rai et al., 2007).

Recent research showed that Drosophila dDNMT2 is also a nuclear protein, which is part of the insoluble nuclear matrix (Schaefer et al., 2008). This finding and data from other species suggest that dDNMT2 may not be exclusively cytoplasmic. The substrate specificity of dDNMT2 enzymes might be broader than previously anticipated, and dDNMT2 might be a DNA/RNA methyltransferase (Jeltsch et al., 2006).

Plants have genes encoding at least three classes of cytosine methyltransferases, namely the MET1 class, the CMT3 (CHROMOMETHYLASE 3) class, and the DOMAINS REARRANGED METHYLASE (DRM) class (Finnegan and Dennis 1993; Henikoff and Comai 1998; Cao et al., 2000).

The MET1 class of genes is most similar to mammalian DNMT1 in both sequence and function. The CMT3 class of genes is specific to the plant kingdom. The CMT3 class is characterized by the presence of a chromodomain amino acid motif between the cytosine methyltransferase catalytic motifs I and IV (Henikoff and Comai 1998; McCallum et al., 2000). MET1 and CMT3 seem to be mainly responsible for the maintenance of DNA methylation at CpG and non-CpG sequences, respectively (Takeda et al., 2006). The third class of genes, composed of DRM1 and DRM2, contain catalytic domains showing sequence similarity to those of the mammalian DNMT3 methyltransferases (Cao et al., 2000). The DRM genes are required for the initial establishment of methylation of cytosines in all known sequence contexts: CpG, CpXpG, and asymmetric (Cao et al., 2002).

\subsubsection{MBD proteins}

The MBD (methyl-CpG binding domain) was identified in MeCP2 (Lewis et al., 1992; Meehan et al., 1992). The minimal MBD possessing methyl-CpG binding activity consists of 85 amino acids (Nan et al., 1993). The structure of the MBD motif of human MBD1 in complex with methylated DNA was resolved by NMR spectroscopy revealing five highly conserved amino acid residues that form a hydrophobic patch mediating the recognition of methyl-CpG dinucleotides (Ohki et al., 2001) (Figure 4). 


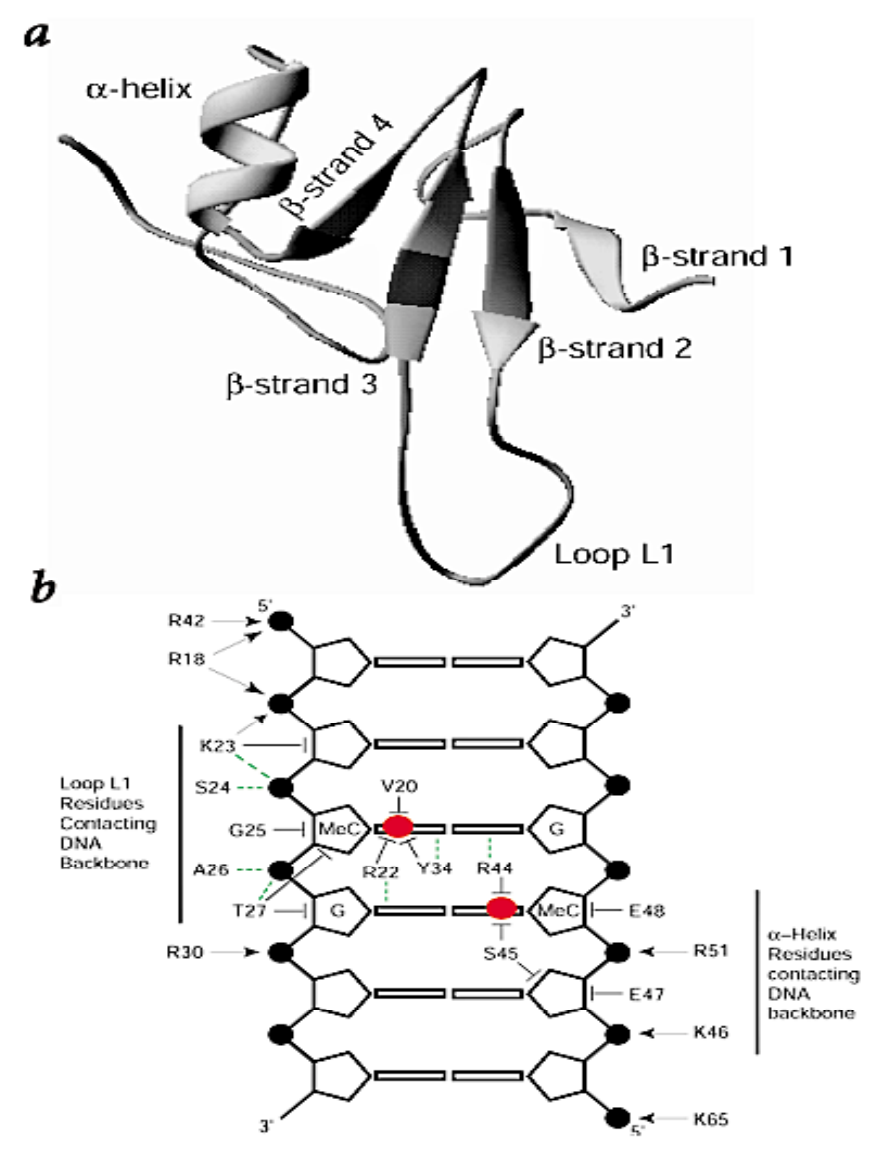

Figure 4. Solution structure of the MBD domain of MBD1. a, Ribbon diagram of the structure. $\boldsymbol{b}$, Protein-DNA contacts. 5-methyl cytosine depicted as a red circle. (Wade et al., 2001).

Humans contain 4 MBD proteins, MBD1-4, in addition to the founding member MeCP2. All but MBD3 specifically recognize and bind methylated CpG sites (Hendrich et al., 2003). In addition to the MBD motif, mammalian MBD1, MBD2 and MeCP2 contain an active transcription repression domain (TRD) capable of long-range repression in vivo (Nan et al., 1997). Besides MBD proteins, a family of proteins named Kaiso-like, which lack the MBD motif, was found to bind methylated $\mathrm{CpG}$ dinucleotides through their zinc-finger domains (Prokhortchouk et al., 2001; Filion et al., 2006). The biological significance of MBD proteins is demonstrated in the Rett syndrome (after Andreas Rett, an Austrian pediatrician who first described this disorder in 1966), a childhood neuro-developmental disorder resulting from mutations in the gene encoding MeCP2 (Amir et al., 1999; Wan et al., 1999). 
Plants also have MBD proteins that are capable of recognizing methylated DNA. The Plant Chromatin database (http://www.chromdb.org/) lists 13 genes encoding putative MBD proteins in Arabidopsis, 17 in rice (Oryza sativa subsp. japonica), 14 in maize (Zea mays) and 14 in poplar (Populus trichocarpa).

The presence of an MBD motif does not necessarily point to methyl-CpG binding activity. Although the Arabidopsis MBD proteins AtMBD5, AtMBD6 and AtMBD7 bind methyl CpG in vitro and localize to highly methylated chromocentres in vivo (and are thus referred to as functional MBD proteins), AtMBD1, AtMBD2, AtMBD4, AtMBD8 and AtMBD11 do not (Zemach et al., 2003; Scebba et al., 2003; Ito et al., 2003). AtMBD5 has been reported to bind 5-methylcytosine in a CpXpX context (Scebba et al., 2003; Ito et al., 2003).

Plants also possess a unique class of MBD proteins not found in animals, the members of which have several MBD motifs. The Plant Chromatin database (http://www.chromdb.org/) lists one such protein in Arabidopsis (AtMBD7), one in poplar (MBD914), one in maize (MBD114) and five proteins in rice (MBD704, MBD705, MBD712, MBD714 and MBD716). In Arabidopsis, for example, AtMBD7 contains three MBD motifs, two of which bind methylated CpG sites in vitro (Zemach et al., 2003).

Plant MBD protein complexes might share common features with mammalian MBD complexes, such as the SWI2/SNF2 chromatin-remodelling factor DDM1 and its association with HDAC activity (Zemach et al., 2003; Zemach et al., 2005), linking DNA methylation with histone modifications.

Drosophila contains 5 candidate MBD proteins (Hendrich and Tweedie 2003). It remains mysterious wheter or not Drosophila MBD protiens bind methylated DNA. For example, several studies suggest that Drosophila dMBD2/3 does not bind to methylated DNA (Tweedie et al., 1999; Ballestar et al., 2001a), and this fits with the fact that the MBD region of the protein is severely disrupted. In contrast, a short form of the protein generated by alternative splicing showed methyl-specific binding activity, even though the splice removed almost half of the MBD (Roder et al., 2000). Another MBD domain protein from Drosophila is CG30426, also called MDU in this thesis. 


\subsection{MBD/SET proteins: bifunctional regulators of gene expression}

\subsubsection{The MBD/SET-domain proteins}

SETDB1 is a human MBD/SET-domain protein (Schultz et al., 2002). The name SETDB1 (SET domain, bifurcated 1) is based on the SET domain being separated by a 347-amino acid insertion that is not seen in other SET-domain proteins. In addition to the divided SET domain, human SETDB1 and its orthologs (ESET of mouse and MDU of Drosophila) also contain the MBD. Recently, all 3 SET-domain proteins were found to be functional H3K9 methyltransferases (Mora-Bermúdez 2002; Schultz et al., 2002; Yang et al., 2002; Seum et al., 2007; Tzeng et al., 2007).

SETDB1/ESET associate with transcription factors, and the association may contribute to the methyltransferase activity of the enzyme. A human ortholog of mAM, a murine ATFaassociated factor, facilitates the ESET-dependent conversion of dimethylated H3K9 to the trimethylated state both in vitro and in vivo (Wang et al., 2003). KAP-1 corepressor for the KRAB-ZFP superfamily of transcriptional silencers binds to SETDB1, and Sumoylated KAP1 stimulates SETDB1's HMT activity (Schultz et al., 2002). Also, ESET associates with ERG, a transcription factor implicated in the control of cell growth and differentiation (Yang et al., 2002).

The function of the MBD domain of SETDB1 remains elusive. However, results support that SETDB1 may connect to DNA methylation in other ways. One way is the direct interaction between SETDB1 and the de novo DNA methyltransferase DNMT3A (Li et al., 2006). Interaction of the two proteins occurs through the $\mathrm{NH}_{2}$-terminus of SETDB1 and the homeodomain of DNMT3A. The other way is the association of SETDB1 with the methyl-CpG binding protein MBD1 (Sarraf and Stancheva 2004), and the MBD1/SETDB1 complex represses transcription through methylation of $\mathrm{H} 3 \mathrm{~K} 9$.

\subsubsection{Gene structure and previous functional studies of $M d u$}

Mdu (CG30426, also called dSETDB1, Egg or dESET) is an annotated Drosophila SET-domain gene, described in the FlyBase Genomic Annotation database (Release 3). This gene is localized on chromosome $2 \mathrm{R}$, and its 2716 -bp long reading frame was predicted to consist of six exons. 


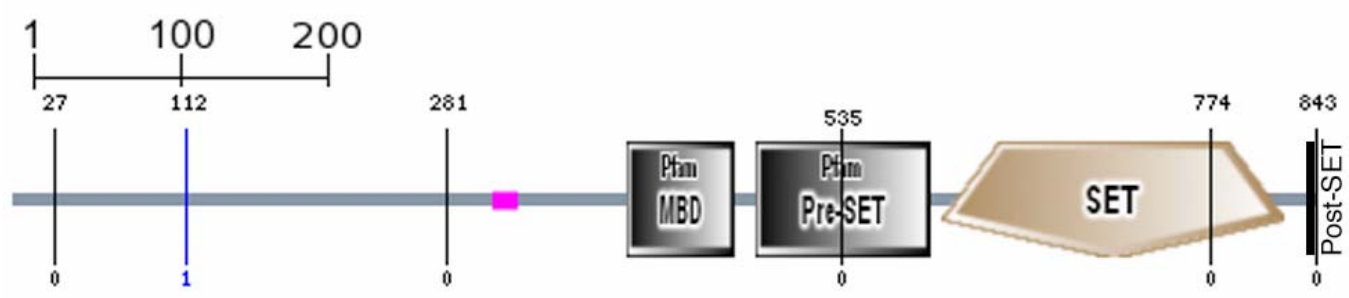

Figure 5. Structure of Mdu (CG30426) gene. MBD, Pre-SET, SET and Post-SET domains are shown.

MDU contains both a MBD domain and a SET domain (Figure 5). The SET domain of MDU is interrupted by a 97 amino acid insertion to create a bifurcated domain. So far, MDU is the only known Drosophila protein that contains a split SET domain. This character is similar to the human SET-domain protein SETDB1 and its mouse ortholog ESET (Ryu et al., 2006). Aligning the mouse and Drosophila SET domains without the spacer sequence revealed $63 \%$ identity. The spacer region, however, is more than three times larger in ESET than in MDU (Figure 6).

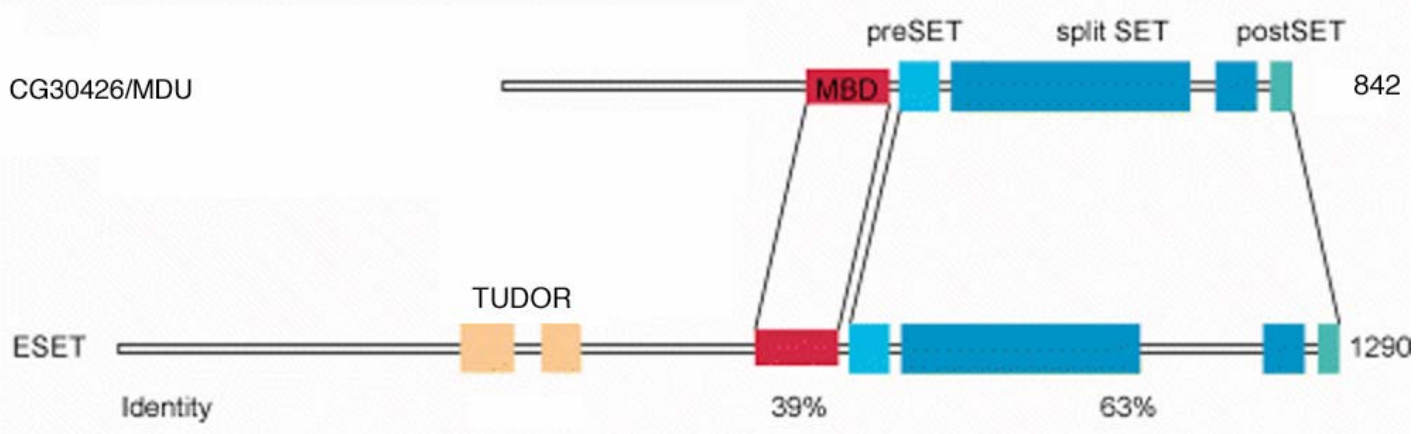

Figure 6. The domain organization of MDU and ESET is conserved. The identity between the domains is indicated in percentages. The schematic proteins are not drawn to scale. Picture adopted from the paper by Starbell (Starbell et al., 2006).

The SET domain of MDU contains two conserved amino acid sequences involved in HMT activity, namely the H(R)FFNHSC (or NHSC) motif (where F represents a hydrophobic 
residue) that is involved in binding of the methyl donor AdoMet (Zhang et al., 2003b), and the consensus post-SET domain (CXCX4C).

Despite some disagreement (Stabell et al., 2006), MDU is generally considered a H3K9 methyltransferase (Mora-Bermúdez 2002; Seum et al., 2007; Tzeng et al., 2007) that targets to the euchromatin and the autosomal chromosome 4 and is an essential factor for chromosome 4 silencing (Seum et al., 2007; Tzeng et al., 2007). MDU is also involved in oogenesis (Clough et al., 2007) and embryo development (Stabell et al., 2006).

Like ESET and SETDB1, MDU contains an MBD. Aligning the mouse and Drosophila MBD domains revealed $39 \%$ identity (Stabell et al., 2006).

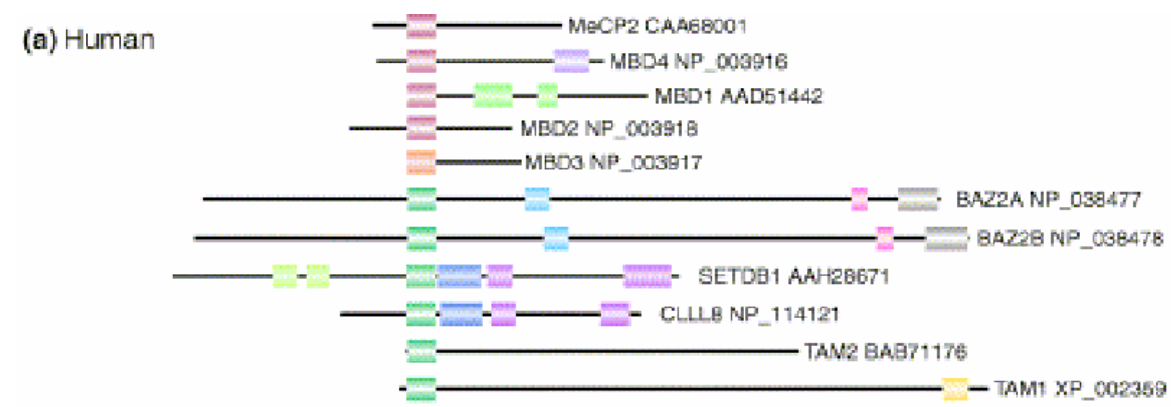

(b) Drosophila
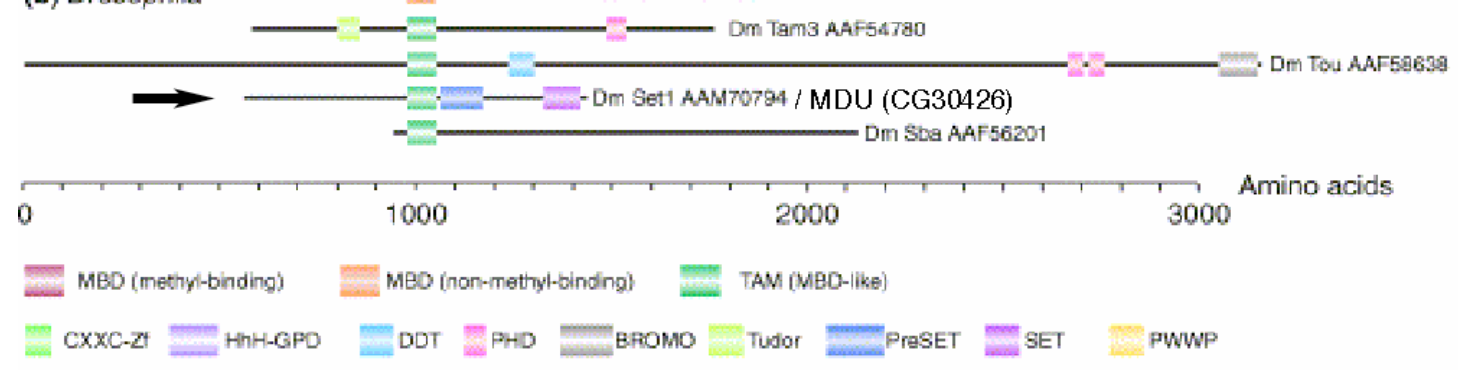

Figure 7. A box diagram showing the conserved sequence motifs as predicted by DART (http://www.ncbi.nlm.nih.gov/Structure/lexington/lexington.cgi?cmd=rps) from the human (a) and Drosophila (b) MBD/TAM containing proteins. Domains known to bind to methylated DNA are shown in red, and those with unknown function are orange. MBDs known to lack methyl-CpG binding activity (MBD3 and DmMBD2/3) are shown in brown. Other conserved domains are indicated. MDU (CG30426) is indicated with an arrow (Hendrich and Tweedie 2003).

MDU and 4 other Drosophila MBD domain proteins are shown in Figure 7 together with human MBD proteins. Other functional domains of these MBD proteins are also shown in this figure. Among these Drosophila MBD proteins, only one, dMBD2/3 has been tested and whether it is a functional methyl DNA binding protein was not determined (Tweedie et al., 1999; Roder et 
al., 2000; Ballestar et al., 2001a). Methyl DNA binding activity has not been investigated in all 4 other MBD proteins, including MDU. To reflect the similarity without implying homology or methyl-binding activity between these untested proteins and the MBD proteins that were shown to bind methyl CpG DNA, a name TAM (for "TIP5, ARBP, MBD") was suggested by Hendrich and Tweedie for these proteins, as shown in Figure 7 (Hendrich and Tweedie 2003).

\subsection{Drosophila Rbf gene}

The human retinoblastoma gene $\mathrm{Rb}$ was the first tumor suppressor gene cloned and is well known as a negative regulator of the cell cycle through its ability to bind the transcription factor E2F and repress transcription of genes required for the $S$ phase (Weinberg 1995). One Drosophila ortholog of $\mathrm{Rb}$ is RBF. E2F ortholog is also found in Drosophila. The Rb-E2F pathway is well conserved and is much simpler in Drosophila than in the mammalian systems (Sutcliffe et al., 2003).

\subsubsection{Mammalian Rb family of proteins}

$\mathrm{Rb}$ and its closely related proteins p107 (RBL1) and p130 (RB2) constitute a protein family that is often referred to as the "pocket proteins" because their main sequence similarity resides in a domain, the pocket domain, that mediates interactions with viral oncoproteins as well as cellular proteins to perform the biological functions of this family (Hinds et al., 1992; Ewen et al., 1993; Endicott 1998). The Rb family of proteins also contains numerous phosphorylation sites that can be phosphorylated by the G1 phase cyclinD/cdk4 complexes and by the G1/S phase cyclinE/cdk2 and cyclinA/cdk2 complexes (Hinds et al., 1992; Ewen et al., 1993; Kato et al., 1993; Resnitzky et al., 1995; Du et al., 1996a).

In general, the hyper-phosphorylated forms of Rb exhibit a decreased ability to interact with their target proteins and to exert their biological functions (Kato et al., 1993; Du et al., 1996a). Because growth-stimulating and growth-inhibitory factors generally affect the transcription, translation and stabilities of the $D$ and $E$ type cyclins, as well as their inhibitors, these growth-signaling pathways regulate cell proliferation, at least in part, through regulating the phosphorylation of the $\mathrm{Rb}$ family of proteins, with the hypo-phosphorylated $\mathrm{Rb}$ being active and preventing transition to the S phase (Chellappan et al., 1991; Kato et al., 1993; Du et al., 1996a). 
The biological functions of $\mathrm{Rb}$ include tumor suppression, regulation of the cell cycle, differentiation and apoptosis (Dannenberg and te Riele 2006). These functions are mediated by Rb's interaction with a large number of cellular proteins. More than 100 proteins have been reported to interact with the $\mathrm{Rb}$ protein (Morris and Dyson 2001), and most, if not all, of these interactions also involve the pocket domain.

The best-studied binding partners of Rb are the E2F transcription factors (Dyson 1998; Attwooll et al., 2004), which function as heterodimers and are composed of a subunit of the E2F and the DP gene families (Attwooll et al., 2004). Mammalian systems contain 8 E2F and 2 DP family members (Dyson 1998; Attwooll et al., 2004).

\subsubsection{Function of "Retinoblastoma Family Proteins" in Drosophila}

Two Rb family genes were found in the Drosophila genome (Dynlacht et al., 1994; Ohtani and Nevins 1994; Du et al., 1996a; Sawado et al., 1998; Stevaux et al., 2002), namely Rbf and Rbf2. Rbf2 is not an essential gene, and Rbf2 mutant flies show no obvious phenotypes (Stevaux et al., 2005). In contrast, RBF appears to fulfill all of the cell cycle-related function of the Rb family of proteins in Drosophila. RBF can bind to both Drosophila E2F proteins (dE2F1 and dE2F2) (Frolov et al., 2001). Characterization of the phenotypes of embryos devoid of RBF revealed that $\mathrm{RBF}$ is required for the repression of E2F target gene expression and for maintaining the first $\mathrm{G} 1$ cell cycle arrest during embryonic development (Du and Dyson 1999). In Drosophila, this establishment of the first G1 cell cycle arrest in the developing embryos requires Dacapo, the only member of the p21/p27 family of cdk inhibitors (De Nooij et al., 1996; Lane et al., 1996). Therefore, $\mathrm{Rb}$ and the $\mathrm{p} 21 / \mathrm{p} 27$ family of cdk inhibitors function cooperatively to achieve a stable G1 cell cycle arrest during fly embryonic development.

In addition to control of the G1/S transition, the Rb-E2F pathway has been implicated in regulating other phases of the cell cycle, as well as cell cycle checkpoints. For example, overexpression of dE2F1 in the Drosophila developing wing accelerates both G1/S and G2/M transitions, whereas overexpression of RBF slowed all phase of the cell cycle, with the greatest effect on S-phase duration (Neufeld et al., 1998). Also, recent screens of Rb/E2F target genes by microarray have identified cell cycle checkpoint genes (Polager et al., 2002; Ren et al., 2002). For example, Mad2, a spindle checkpoint gene, was recently identified as an E2F target. Removal of Rb or overexpression of E2F1 was sufficient to upregulate the expression of Mad2 throughout the cell cycle. Significantly, partial suppression of Mad2 level was sufficient to reverse the chromosomal abnormalities associated with removal of $\mathrm{Rb}$ (Hernando et al., 2004). 
$\mathrm{Rb}$ has both direct and indirect roles in suppressing apoptosis. A role for $\mathrm{Rb}$ and $\mathrm{E} 2 \mathrm{~F}$ in regulating apoptosis was observed in Drosophila, whereby overexpression of dE2F1 or removal of RBF all led to increased apoptosis (Asano et al., 1996; Du et al., 1996b; Du and Dyson 1999).

$\mathrm{Rb}$ and $\mathrm{E} 2 \mathrm{~F}$ proteins are transcriptional regulators that control transcription through a variety of mechanisms. E2F alone can activate transcription, whereas binding by Rb not only blocks transcriptional activation but also leads to active repression.

One mechanism of $\mathrm{Rb}$ repression is through recruitment of co-repressors. $\mathrm{Rb}$ was first shown to interact with HDAC1 through its pocket domain (Brehm et al., 1998). Rb that has been phosphorylated by cyclinD/cdk4 cannot bind to HDACs, which allows the expression of targets such as cyclin $\mathrm{E}$ (Zhang et al., 2000).

$\mathrm{Rb}$ may also repress transcription through its ability to recruit HMTs (Nielsen et al., 2001). Endogenous Rb associates with SUV39H1, an enzyme that methylates lysine 9 on histone H3. Additionally, HP1, a protein that binds methylated lysine 9 and is associated with transcriptionally silent regions of chromatin, can bind $\mathrm{Rb}$ (Nielsen et al., 2001). Furthermore, Rb interacts with the DNA methyltransferase enzyme DNMT1, which cooperates to repress reporter genes (Robertson et al., 2000). However, DNA methylation was not detected at the repressed promoters, which suggests that the effect of DNMT1 may not be through its enzymatic activity but instead, it might help recruit other co-repressors.

\subsubsection{Regulation of RBF activity}

The best-characterized functional regulation of $\mathrm{Rb}$ is the phosphorylation of the protein. $\mathrm{Rb}$ family members are post-translationally regulated, and their gradual phosphorylation leads to their functional inactivation. In the early G1 phase, D-type cyclins interact with the A/B pocket of the Rb gene products via their LXCXE motif; in particular, they couple with the kinases cdk4 or cdk6 and phosphorylate pRb/105 and Rb2/p130 (Dowdy et al., 1993). In the middle to late G1 phase, cyclins $E$ and $A$ form complexes with cdk2 to specifically target Rb/p105, Rb2/p130 and p107 (De Luca et al., 1997). This step is crucial for the inactivation of members of the pRb family and the release of E2F factors.

Phosphorylation of $\mathrm{Rb}$ can cause permanent inactivation and lead to degradation (Tedesco et al., 2002). High levels of cdk activity may result in phosphorylation of pRb-Ser567, which exposes the $\mathrm{Rb}$ protein to a proteolytic cleavage site and degradation by an unidentified protease (Ma et al., 2003). 
Pocket protein phosphorylation is often reversed by dephosphorylation. Pocket protein dephosphorylation is known to take place from anaphase to $\mathrm{G} 1$ and to depend upon protein phosphatase 1 (Ludlow et al., 1993); dephosphorylation occurs in response to growth inhibitory signals.

The $\mathrm{COOH}$-terminal region of $\mathrm{Rb}$ proteins also plays an important role in orchestrating the activity of these proteins. The carboxy-terminal region is quite important because it contains the nuclear localization signal (NLS) and domains responsible for HDAC1 and cyclin/cdk complex binding. The NLS controls the transport of $\mathrm{Rb}$ proteins from the cytoplasm to the nucleus (Efthymiadis et al., 1997).

The activity of $\mathrm{Rb}$ is also regulated at the epigenetic level. Actually, the first link between DNA hypermethylation and tumour-suppressor genes was determined with the example of $R b$, the first known tumour-suppressor gene. Dryja and Horsthemke's laboratories were the first to indicate that tumour-suppressor silencing might occur by an epigenetic pathway (Greger et al., 1989; Sakai et al., 1991). In the Horsthemke study in 1989, hypermethylation was specifically linked to $\mathrm{Rb}$, which led this group to suggest that DNA hypermethylation might have a direct role in tumour-suppressor gene silencing (Greger et al., 1989). In 1991, Dryja's group showed that the hypermethylation of $\mathrm{Rb}$ was confined to one allele, which also indicates the specificity of this epigenetic event. This hypermethyation may lead to gene silencing (Sakai et al., 1991). Later, direct confirmation of epigenetic silencing of a tumour-suppressor gene was provided by Sakai's group, who showed a $92 \%$ reduction of $\mathrm{Rb}$ expression in tumours with promoter hypermethylation (Ohtani-Fujita et al., 1993).

Recently, the correlation of DNA hypermethylation and Rb gene silencing was observed in Drosophila. Epigenetic silencers and the Notch signaling pathway were found to collaborate to promote malignant eye tumours in Drosophila by silencing the Drosophila Rb ortholog Rbf. Transcription of Rbf was downregulated in these tumours, and this downregulation was associated with DNA hypermethylation of the enhancer and exon 1 of Rbf (Ferres-Marco et al., 2006).

Despite all the observations of Rb hypermethylation, the molecular mechanism of this methylation is still not clear. CCCTC-binding factor (CTCF) might be involved in the regulation of Rb-promoter DNA methylation (De La Rosa-Velázquez et al., 2007). CTCF protects the Rb gene promoter against DNA methylation, and when this control region is abnormally methylated, a methyl-CpG binding protein, Kaiso, and probably its associated repressor complex, induce epigenetic silencing of the promoter. However, how methylation is initiated at the $R b$ gene in the first place has not been determined. 


\subsection{Specific aims of this study}

The Post-translational modification of DNA and histones plays an important role in regulating the structure and function of chromatin to activate or repress gene activity. DNA and histone methylation have been associated with epigenetic phenomena such as imprinting, gene dosage compensation and gene silencing.

Epigenetic silencing of gene expression involves an intricate interplay between DNA methyltransferases, methyl-CpG binding proteins, HMTs, histone acetyltransferase (HATs) and histone deacetylase (HDACs) (Dobosy and Selker 2001; Bannister and Kouzarides 2004; Herceg and Wang 2005). DNA and histone methylation, particularly methylation of lysine 9 or 27 in histone $\mathrm{H} 3$, are functionally closely interconnected. DNA methylation patterns can initiate subsequent histone modifications (such as histone deacetylation and methylation) during gene silencing (Fuks 2003b), whereas other studies suggest that histone methylation and deacetylation precede and trigger DNA methylation (Tamaru and Selker 2001; Strunnikova et al., 2005).

Epigenetic gene silencing plays a fundamental role in the control of cell proliferation and differentiation, and mutations in components of the enzymatic machineries mediating epigenetic gene silencing have been correlated with various human diseases including cancer and Rett syndrome. Consequently, the functional dissection of the players and mechanisms underlying epigenetic gene silencing is important to advance our knowledge of the molecular mechanisms that control cell differentiation and proliferation in development and disease.

In contrast to mammals, little is known about the role of DNA methylation in model systems such as $D$. melanogaster. The fly contains key components of the enzymatic machineries involved in methylation of DNA and histones; however how DNA methylation contributes to the execution of epigenetic events in Drosophila and cooperates with other epigenetic signals such as histone methylation remains unclear. DNA methylation of the fly genome was discovered in 2000 (Lyko et al., 2000a; Gowher et al., 2000). Despite significant progress, several aspects of the DNA methylation machinery such as the factors triggering DNA methylation remain unknown.

Previous studies in our lab revealed that the MBD/SET protein MDU has HMT-activity and methylates lyine 9 in histone H3. Methylation of H3K9 has been associated with epigenetic gene silencing, suggesting that MDU is a putative epigenetic repressor. The presence of a MBD 
domain in MDU raised the possibility that MDU is not only involved in $\mathrm{H} 3 \mathrm{~K} 9$ methylation but also in the DNA methylation machinery of the fly.

In this study, I have investigated the role of MDU in $\mathrm{H} 3 \mathrm{~K} 9$ and DNA methylation in epigenetic gene silencing in Drosophila. To achieve this goal, I used different methodologies in molecular biology and biochemistry, tissue culture cell assays, and Drosophila genetics.

To dissect the role of MDU in histone and DNA methylation in vitro and in vivo, antibodies against key components of the Drosophila DNA machinery and MDU were generated.

In vitro DNA pull-down assays were used to assess whether the MBD of MDU interacts with methyated DNA.

In vitro HMT-assays coupled to Western blot analysis were used to investigate the product specificity of the HMT-activity within the SET-domain of MDU. The transcriptional regulatory potential of MDU was studied in transient transfection assays in Drosophila tissue culture cells.

Target genes for MDU were identified by the use of DNA immuoprecipitation assays. One of the identified target genes is the "retinoblastoma family protein" (Rbf). A recent study had correlated DNA hypermethylation with the silencing of Drosophila tumor-suppressor gene Rbf (Ferres-Marco et al., 2006). Chromatin immunoprecipitation and reverse-transcription coupled to PCR (RT-PCR) assays were used to assess the role of MDU in silencing of Rbf expression in Drosophila tissue culture cells. RNA interference assays monitored the role of MDU in eye development.

The results of this study reveal that MDU plays an important role in gene silencing in Drosophila. MDU-mediated methylation of H3K9 triggers de novo DNA methylation at target genes, culminating in epigenetic repression. The results uncover a mechanism for de novo DNA methylation in Drosophila and the importance of MBD/SET proteins in epigenetic gene silencing. The results support a model in which histone and DNA methylation by MBD/SET proteins control cell proliferation and differentiation in development and disease. 


\section{Materials and Methods}

\subsection{Materials}

\subsubsection{Laboratory Equipment}

Autoradiography developer

Cell counting chamber

Centrifuge 5415D

Centrifuge 5810R

Centrifuge Biofuge pico

Chromatography columns

Class II A2 Biological Safety Cabinet

Computer, Hardware

Computer, Software:

Custom DNA-gel system

Eagle Eye II still video system

Electronic Pipettes

FACSAria cell sorter

Freezer $-20^{\circ} \mathrm{C}$

Freezer $-80^{\circ} \mathrm{C}$

Fridge $4-10^{\circ} \mathrm{C}$

Gel Dryer Vacuum system

Heatblock

Ice machine

Ice-bath

Innova 4230 refrigerated incubator shaker

Intensification cassettes for autoradiography

LB-122 $\beta$ - $\gamma$-detector

Low Temperature Incubator

LSC Homogenizer LH-22

Luminometer

Magnetic stirrer / heat plate

Microscope

Microwave oven

Milli-Q Synthesis water purification system

Mini Trans-Blot transfer cell

Nutator
Amersham Pharmacia Biotech

Neubauer

Eppendorf

Eppendorf

Heraeus

Pharmacia Biotech

ThermoForma

iMac, $800 \mathrm{MHz}$

Microsoft Excel

Microsoft Word

Microsoft Powerpoint

Mozilla Firefox Internet Browser

Lasergene Navigator, DNAStar

Adobe Photoshop 5.0

DNA Strider 1.3

ZMBH Heidelberg, Workshop

Stratagene

Brandt

Becton, Dickinson and Company

Frigidaire

ThermoForma

Frigidaire

Fisher Scientific

Techne Dri-block DB-2A

Scotsman

Neolab

New Brunswick Scientific

Suprema, Dr. Goos

Berthold

Fisher Scientific

Yamato

GloMax

Fisher Scientific

Nikon

Sharp Carousel

Millipore

BioRad

Clay Adams Brand 
pH-meter (accumet ${ }^{\circledR}$ basic AB15)

Pipettes (Pipetman: 2, 10, 20, 200, $1000 \mu \mathrm{l}$ size)

Power supplies

Printer HP Laserjet 4100

Protean 3 minigel system

PTC-100 Peltier Thermal Cycler

PTC-200 Peltier Thermal Gradient Cycler

Quarz Cuvette $(1 \mathrm{~cm}, \mathrm{Z}=8.5)$

QuixSep Micro Dialyzer dialysis chamber

$\mathrm{RC}-5$ (B) refrigerated superspeed centrifuge

Rocker/shaker Roto Shake Genie

Scale

Scale (analytical)

Scanner

Scintillation Counter

Shaker (horizontal)

SmartSpec ${ }^{\text {TM }}$ Plus spectrophotometer

Sonifier 450

Spectrophotometer

Spinner flasks for cell culture

Thermomixer

Tweezers

Vortex Genie 2

Waterbath SUB 14

Zip-drive

\subsubsection{Consumables and Kits}

$10 \mathrm{~cm}$ cell culture dishes

$100 \mathrm{ml}$ cell culture flask (with filter cap)

$15 \mathrm{~cm}$ cell culture dishes

$15 \mathrm{ml}$ tubes

$50 \mathrm{ml}$ tubes

6-well plates for cell culture

Chromatography column

Dneasy Blood \& Tissue Kit

DIG RNA labeling Kit

ECL-Plus Western Blotting Detection System

EpiTect Bisulfite Kit

Filter pipette tips $(10,20,200,1000 \mu l$ size $)$

Filterpaper

Glass beads (425-600 micron)

Luciferase Assay System (with 5x lysis buffer)

PCR tube, $0.2 \mathrm{ml}$

Microtube, $1.5 \mathrm{ml}$

Parafilm "M"

Perfectprep ${ }^{\mathrm{TM}}$ Gel Cleanup Kit

Pipet tips (10, 20, 200, $1000 \mu \mathrm{l})$

QiagenMaxi Kit
Fisher Scientific

Gilson

Power Pac 300, BioRad

Hewlett Packard

BioRad

MJ Research

MJ Research

Sarna Cells

Membrane Filtration Products Inc.

Sorvall

Scientific Industries Inc.

Fisher Scientific

Fisher Scientific

Epson

Beckman

GFL 3005

BioRad

Branson

SmartSpecPlus, BioRad

Wheaton, Bellco

Eppendorf

Roth, Karlsruhe

Scientific Industries Inc.

Grant

lomega
Greiner

Greiner

Greiner

Fisher

Fisher

Greiner

Bio-rad

Qiagen

Roche

Amersham

Qiagen

Axygen

Whatman

Sigma

Promega

Axygen

Axygen

American National Can

Eppendorf

Axygen

Qiagen 
QiagenMini Kit

Qiaquick Nucleotide Removal Kit

Quick Ligation Kit

QuikChange $^{\text {TM }}$ Site-Directed Mutagenesis Kit

RNA Midiprep Kit

Scintillation fluid

Serological pipettes

Sterile filters $(20 \mu \mathrm{m}$ and $45 \mu \mathrm{m})$

Sterile filtration units

Streptavidine

TOPO TA cloning kit

$\mathrm{X}-\mathrm{OMAT}^{\mathrm{TM}}$ autoradiography film
Qiagen

Qiagen

NEB

Stratagene

Qiagen

Betamax

Falcon

Millipore

Nalgene

BioRad

Invitrogen

Kodak

\subsubsection{Chemicals, Enzymes, Proteins and Molecular Weight Markers}

Chemicals not listed were of P. A. quality and were purchased from the companies Sigma (St. Louis, MO), Fisher Scientific (Hampton, NH) and VWR (West Chester, PA).

Acrylamide (40\% stock)

Agarose (ultra pure)

APS (ammoniumpersulfate)

Baculovirus DNA

BCIP (5bromo-4chloro-3indolyl-phosphate)

Bromophenol Blue (Na-salt)

Cellfectin

Citric Acid

Coomassie ${ }^{\circledR}$ R250 Brilliant Blue

Deep Vent Polymerase

DTT

EDTA

Ethanol 95\%

Ethidiumbromide

GeneRuler 1kb DNA-Ladder

Glucose

Glycerine (Glycerol)

Glycogen

Guanidine hydrochloride

Hepes (N-Cyclohexyl-2-aminoethanesulfonic acid)

Histone-octameres

Hotmaster Taq Polymerase

Isopropanol (2-propanol)

L-Arginine free base

L-Glutamin/Penicillin/Streptomycin-Mix

LB Broth, Miller

LB-Agar

Lysozyme

Methanol
Fisher

VWR

Merck

Baculo Gold, Pharmingen

Boehringer Mannheim

Sigma

Invitrogen

VWR/EMD

Serva

NEB

AppliChem $\mathrm{GmbH}$

VWR

Biochemistry Dpt., UC Riverside

ICN Biomedicals Inc.

MBI Fermentas

MP Biomedicals

VWR/EMD

J.T. Baker

VWR/EMD

Fisher

F. Sauer

Eppendorf

VWR

VWR/EMD

Gemini Bioproducts

VWR

VWR

Roche

VWR 
NAMP100V Amplify

NBT (nitro blue tetrazolium chloride)

Neomycin, $0.5 \mathrm{mg} / \mathrm{ml}$

NONIDET ${ }^{\circledR}$ P40

Nucleosomes

Pipes (Piperazine-1,4-bis[2-ethanesulfonic acid])

Pluronic ${ }^{\circledR}$ F-68

PMSF

Ponceau-S protein staining solution

Potassiumtetrathionate

Protein marker, broad range

Protein marker, prestained

Proteinase K

Quick Ligase

Restriction enzymes and buffers

RNasin, ribonuclease inhibitor

RNaseA

Salmon testis DNA

SDS (sodiumdodecylsulfate)

Shrimp alkaline phosphatase

Sodiumchloride

sodiumdesoxycholate

Skim milk powder

SF900Il serum-free insect cell media

Sodiumhydroxide

Superscript RT

TaKaRa ${ }^{\text {Ex}}$ Taq Polymerase

TEMED

Tryptone

Tween ${ }^{\circledR} 20$

Yeast extract
Amersham

Boehringer Mannheim

Sigma

Calbiochem

F. Sauer

Fisher

Gibco

Roche

Serva

Pfaltz \& Bauer Inc.

NEB

NEB

Roche

NEB

NEB

Promega

Roche

Sigma

VWR

Boehringer Mannheim

VWR

Fisher

SACO Foods Inc.

Gibco

VWR/EMD

Invitrogen

TaKaRa

Fisher

VWR/EMD

Fisher

Gibco

\subsubsection{Antibodies and Affinity Matrixes}

Optitran reinforced NC membrane

Westran PVDF-Membrane

Immobilon-P (PVDF) Membrane

Nitrocellulose membrane

Glutathione sepharose

Anti-FLAG M2 affinity agarose

Protein-A-agarose

Protein-G-Agarose

Anti-5-methyl cytosine Polyclonal Antibody, rabbit

Anti-mono-methyl(H3K9), rabbit

Anti-di-methyl(H3K9), rabbit

Anti-tri-methyl(H3K9), rabbit

Anti-dDNMT2, rabbit

Anti-MDU (CG30426), rat
Schleicher \& Schüll

Schleicher \& Schüll

Millipore

Millipore

Amersham

Sigma

Amersham

Upstate

Megabase

Abcam

Abcam

Abcam

Dawei Gou/Biosynthesis

Dawei Gou/ Elisabeth Kremmer 
Anti-rat-lgG-AP-conjugate

Sigma

Anti-rabbit-IgG-AP-conjugate

Sigma

Anti-rabbit-biotinylated

Amersham

\subsubsection{Radioactive substances}

Redivue adenosine-5'-[ $\left.\gamma_{-}-{ }^{32} \mathrm{P}\right]$-triphosphate,

Amersham

Triethylammonium salt $10 \mathrm{mCi} / \mathrm{ml}$

s-Adenosyl-I-[methyl-3H]methionine

Amersham

2.2-3.1 TBq/mmol, $15 \mathrm{Ci} / \mathrm{mmol}$.

\subsubsection{Bacteria Stocks}

XL-1 blue (Bullock et al., 1987); Stratagene

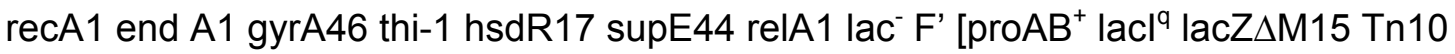
$\left(\right.$ Tet $\left.\left.^{r}\right)\right]$

BL21 (DE3)pLysS; Novagen

$\mathrm{F}-$ ompt hsdSB $\left(\mathrm{rB}^{-} \mathrm{mB}^{-}\right)$gal dcm (DE3) pLysS (CmR)

\subsubsection{Insect Cells}

Sf9 insect cells; Orbigen Inc.

Cell line derived from pupal ovarian tissue of Spodoptera frugiperda (fall armyworm). Sf9 cells were used to isolate and propagate recombinant baculoviral stocks and to produce recombinant proteins (Smith et al., 1985; Vaughn et al., 1977).

Schneider S2 cells; Invitrogen

Cell line derived from Drosophila melanogaster (fruit fly; wild type-strain "Oregon R") dissociated, near hatching embryos. S2 cells were used to produce recombinant proteins and for XChIP experiments (Schneider 1972).

S2-tet-tk-luc cells; Talay and Sauer, 2004

Derivate of Schneider S2 cells with a recombinant fragment (tet-tk-luc) integrated into its genome (Talay 2004). The integrated fragment contains tetO-operator, thymidine-kinase promotor and luciferase reporter gene arranged in the order as listed. S2-tet-tk-luc cells were used in the luciferase reporter assay to demonstrate the transcription regulation activity of certain factors. 


\subsubsection{Oligonucleotides}

Oligonucleotides (oligos) were produced by Sigma-Genosys in desalted purity grade. All sequences are shown in 5'-3' orientation.

\subsubsection{Oligonucleotides for DNA pull-down assay}

$5 \mathrm{mC}$ is $5-$ methyl cytosine

\begin{tabular}{|l|l|}
\hline CG-5 & GATCCGACGACGACGACGACGACGACGACGACGACGACGATC \\
\hline CG-3 & GATCGTCGTCGTCGTCGTCGTCGTCGTCGTCGTCGTCGGATC \\
\hline $5 \mathrm{mCG}-5$ & GATCCGACGACGACGACGA5mCGACGACGACGACGACGACGATC \\
\hline $5 \mathrm{mCG}-3$ & GATCGTCGTCGTCGTCGTCGT5mCGTCGTCGTCGTCGTCGGATC \\
\hline CA-5 & GATCCGACGACGACGACGACATGACGACGACGACGACGACGATC \\
\hline CA-3 & GATCGTCGTCGTCGTCGTCGTCATGTCGTCGTCGTCGTCGGATC \\
\hline $5 \mathrm{mCA}-5$ & GATCCGACGACGACGACGA5mCATGACGACGACGACGACGACGATC \\
\hline $5 \mathrm{mCA}-3$ & GATCGTCGTCGTCGTCGTCGT5mCATGTCGTCGTCGTCGTCGGATC \\
\hline CT-5 & GATCCGACGACGACGACGACTAGACGACGACGACGACGACGATC \\
\hline CT-3 & GATCGTCGTCGTCGTCGTCGTCTAGTCGTCGTCGTCGTCGGATC \\
\hline $5 \mathrm{mCT}-5$ & GATCCGACGACGACGACGA5mCTAGACGACGACGACGACGACGATC \\
\hline $5 \mathrm{mCT}-3$ & GATCGTCGTCGTCGTCGTCGT5mCTAGTCGTCGTCGTCGTCGGATC \\
\hline CCA-5 & GATCCGACGACGACGACGACCATGGACGACGACGACGACGACGATC \\
\hline CCA-3 & GATCGTCGTCGTCGTCGTCGTCCATGGTCGTCGTCGTCGTCGGATC \\
\hline C5mCA-5 & GATCCGACGACGACGACGAC5mCATGGACGACGACGACGACGACGATC \\
\hline C5mCA-3 & GATCGTCGTCGTCGTCGTCGTC5mCATGGTCGTCGTCGTCGTCGGATC \\
\hline CCT-5 & GATCCGACGACGACGACGACCTAGGACGACGACGACGACGACGATC \\
\hline CCT-3 & GATCGTCGTCGTCGTCGTCGTCCTAGGTCGTCGTCGTCGTCGGATC \\
\hline C5mCT-5 & GATCCGACGACGACGACGAC5mCTAGGACGACGACGACGACGACGATC \\
\hline C5mCT-3 & GATCGTCGTCGTCGTCGTCGTC5mCTAGGTCGTCGTCGTCGTCGGATC \\
\hline
\end{tabular}

\begin{tabular}{|l|l|}
\hline Tri-CG-5 & GATCCGACGACGACGACGACGACGACGACGACGACGACGATC \\
\hline Tri-CG-3 & GATCGTCGTCGTCGTCGTCGTCGTCGTCGTCGTCGTCGGATC \\
\hline Tri-5mCG-5 & GATCCGACGACGA5mCGA5mCGA5mCGACGACGACGACGACGACGATC \\
\hline Tri-5mCG-3 & GATCGTCGTCGTCGTCGTCGT5mCGT5mCGT5mCGTCGTCGTCGGATC \\
\hline Tri-CA-5 & GATCCGACGACGACATGACATGACATGACGACGACGACGACGACGATC \\
\hline Tri-CA-3 & GATCGTCGTCGTCGTCGTCGTCATGTCATGTCATGTCGTCGTCGGATC \\
\hline Tri-5mCA-5 & GATCCGACGACGA5mCATGA5mCATGA5mCATGACGACGACGACGACGACGATC \\
\hline Tri-5mCA-3 & GATCGTCGTCGTCGTCGTCGT5mCATGT5mCATGT5mCATGTCGTCGTCGGATC \\
\hline Tri-CT-5 & GATCCGACGACGACTAGACTAGACTAGACGACGACGACGACGACGATC \\
\hline Tri-CT-3 & GATCGTCGTCGTCGTCGTCGTCTAGTCTAGTCTAGTCGTCGTCGGATC \\
\hline Tri-5mCT-5 & GATCCGACGACGA5mCTAGA5mCTAGA5mCTAGACGACGACGACGACGACGATC \\
\hline Tri-5mCT-3 & GATCGTCGTCGTCGTCGTCGT5mCTAGT5mCTAGT5mCTAGTCGTCGTCGGATC \\
\hline Tri-CCA-5 & GATCCGACGACGACCATGGACCATGGACCATGGACGACGACGACGACGACGATC \\
\hline Tri-CCA-3 & GATCGTCGTCGTCGTCGTCGTCCATGGTCCATGGTCCATGGTCGTCGTCGGATC \\
\hline Tri-C5mCA-5 & GATCCGACGACGAC5mCATGGAC5mCATGGAC5mCATGGACGACGACGACGACGACGATC \\
\hline Tri-C5mCA-3 & GATCGTCGTCGTCGTCGTCGTC5mCATGGTC5mCATGGTC5mCATGGTCGTCGTCGGATC \\
\hline Tri-CCT-5 & GATCCGACGACGACCTAGGACCTAGGACCTAGGACGACGACGACGACGACGATC \\
\hline Tri-CCT-3 & GATCGTCGTCGTCGTCGTCGTCCTAGGTCCTAGGTCCTAGGTCGTCGTCGGATC \\
\hline Tri-C5mCT-5 & GATCCGACGACGAC5mCTAGGAC5mCTAGGAC5mCTAGGACGACGACGACGACGACGATC \\
\hline Tri-C5mCT-3 & GATCGTCGTCGTCGTCGTCGTC5mCTAGGTC5mCTAGGTC5mCTAGGTCGTCGTCGGATC \\
\hline
\end{tabular}




\subsubsection{Oligonucleotides for inserting point-mutations with PCR}

Mutated codons are shown in bold letters.

\begin{tabular}{|l|l|}
\hline 5'-MDUmSET-H775L & GGACGCTATTTCAACCTCTCGTGCAGCCCC \\
\hline 3'-MDUmSET-H775L & GGGGCTGCACGAGAGGTTGAAATAGCGTCC \\
\hline 5'-MDUmMBD-R436C & GTGGCAAAAGCTTGTGCAGCCTGGCCGAAGTTC \\
\hline 3'-MDUmMBD-R436C & GAACTTCGGCCAGGCTGCACAAGCTTTTGCCAC \\
\hline
\end{tabular}

\subsubsection{Oligonucleotides for cloning}

The positions of restriction sites are underlined and the specific enzyme is shown in brackets.

\begin{tabular}{|l|l|}
\hline $\begin{array}{l}\text { 5'-pVLFLAG-MDU } \\
\text { (Nde1) }\end{array}$ & GGAATTCCATATGCACTGCGCTTTGTATAACTCGTGTCCG \\
\hline $\begin{array}{l}\text { 3'-pVLFLAG-MDU } \\
\text { (BamH1) }\end{array}$ & GACGCGGATCCTTAGAGCAGACGAAGGCGGCAATTGGG \\
\hline $\begin{array}{l}\text { 5'-pVLFLAG-ANMDU } \\
\text { (Nde1) }\end{array}$ & GGAATTCCATATGAACCATACGGAGTGGATATACAGGGGT \\
\hline 5'-pGEX-MBD (Nco1) & CTTCATGCCATGGGCTACAGTCCGTTAGCGAAGCCTCTG \\
\hline 3'-pGEX-MBD (Xba1) & CTAGTCTAGATTAAATGGAGTACTCGGCCAGACACTTGAGG \\
\hline $\begin{array}{l}\text { 5'-pBSFLAG-MDU } \\
\text { (Nde1) }\end{array}$ & GGAATTCCATATGCACTGCGCTTTGTATAACTCG \\
\hline $\begin{array}{l}\text { 3'-pBSFLAG-MDU } \\
\text { (Xba1) }\end{array}$ & GGAATTCTCTAGATTAGAGCAGACGAAGGCGGCAATTGGG \\
\hline $\begin{array}{l}\text { 5'-pPAc-tetRMDU } \\
\text { (BamH1) }\end{array}$ & GACCCCGGATCCACCGGTCGCCACCATGGCTGACTACAAGGAC \\
\hline $\begin{array}{l}\text { 3'-pPAc-tetRMDU } \\
\text { (Not1) }\end{array}$ & GTACGTACGCGGCCGCTTAGAGCAGACGAAGGCG \\
\hline 5'-pPAc-MDU (BamH1) & CGCGGATCCACCGGTCGCCACCATGGTGATGCACTGCGCTTTGTAT \\
\hline 3'-pPAc-MDU (Not1) & GTACGTACGCGGCCGCTTAGAGCAGACGAAGGCG \\
\hline $\begin{array}{l}\text { 5'-pVLFLAG-dDNMT2 } \\
\text { (Nde1) }\end{array}$ & CATGCATGCATGCATATGCATTATGCCTTTAATTAT \\
\hline $\begin{array}{l}\text { 3'-pVLFLAG-dDNMT2 } \\
\text { (Not1) }\end{array}$ & GTACGTACGCGGCCGCTTATTTTATCGTCAGCAATTT \\
\hline $\begin{array}{l}\text { 5'-PCRTOPO- } \\
\text { MDU500bp }\end{array}$ & AAAGAGGGATACGAGTCGGAGGTGGACCAC \\
\hline $\begin{array}{l}\text { 3'-PCRTOPO- } \\
\text { MDU500bp }\end{array}$ & TTAGAGCAGACGAAGGCGGCAATTGGGG \\
\hline $\begin{array}{l}\text { 5'-PCRTOPO- } \\
\text { RBF500bp }\end{array}$ & CCTAAACGTAACTCCGGACGTGAGTGGCCG \\
\hline $\begin{array}{l}\text { 3'-PCRTOPO- } \\
\text { RBF500bp }\end{array}$ & ATAAAATAGTCGAACGTGCGCCGGAGGGGC \\
\hline
\end{tabular}




\subsubsection{Oligonucleotides for sequencing (5'-3')}

\begin{tabular}{|l|l|}
\hline pGEX-fwd & GGGCTGGCAAGCCACGTTTGGTG \\
\hline pPAc-fwd & TTGTGCTGTGTGGATACTCC \\
\hline T7 & TAATACGACTCACTATAGGG \\
\hline M13 (forward) & GTAAAACGACGGCCAG \\
\hline M13 (reverse) & CAGGAAACAGCTATGAC \\
\hline MDUreverse600 & CTTCTCCACGTACTTCTG \\
\hline MDUreverse1002 & ATTGGTGCGTCCTCCGGCTGGG \\
\hline MDUreverse1592 & TCGCAGTCGCAGCACAAGAG \\
\hline MDUreverse2083 & GGAAGTCGTCATCGTCCTCG \\
\hline MDUfwd1951 & GCGTTAACGACATCCC \\
\hline MDUfwd2183 & GTGCGGTGATCAACTTTAAC \\
\hline dDNMT2reverse243 & ACAGATGAGTAAGTGCATCCG \\
\hline dDNMT2fwd10 & GCCTTTAATTATGCCCAATTG \\
\hline dDNMT2fwd546 & CGAGATTGTGGAGGAAAATG \\
\hline
\end{tabular}

\subsubsection{Oligonucleotides for RT-PCR reactions}

\begin{tabular}{|l|l|}
\hline RT-RBF-5 & CCTAAACGTAACTCCGGACGTGAGTGGCCG \\
\hline RT-RBF-3 & ATAAAATAGTCGAACGTGCGCCGGAGGGGC \\
\hline RT-MDU-5 & AAAGAGGGATACGAGTCGGAGGTGGACCAC \\
\hline RT-MDU-3 & TTAGAGCAGACGAAGGCGGCAATTGGGG \\
\hline RT-dDNMT2-5 & GTTTCGAGAGCTCACAGGCGCGAAATCAGT \\
\hline RT-dDNMT2-3 & GCAACAGGTCCAAGCGTTGCTGCAAAATCT \\
\hline RT-ACTIN-5 & CGTTCTGGACTCCGGCGATGG \\
\hline RT-ACTIN-3 & GTACTTGCGCTCTGGCGGGGC \\
\hline
\end{tabular}

\subsubsection{Oligonucleotides for XChIP-reactions}

\begin{tabular}{|l|l|}
\hline RBFenhancer-5 & CATCTTTTAATGCCCTCTGCGACG \\
\hline RBFenhancer-3 & ATGACTCTGCCAAGTGACCTCCAG \\
\hline RBFexon1-5 & CAGAAAAGGGTGTATCGCGAACAC \\
\hline RBFexon1-3 & CTTTTAGGCAGGCAATGCACAGAA \\
\hline tetO-TK-LUC-5 & ACCCGGGTACCGAGCTCG \\
\hline tetO-TK-LUC-3 & TTATGTTTTTGGCGTCTTCCATGG \\
\hline
\end{tabular}

\subsubsection{Oligonucleotides for Bisulfite sequencing}

\section{a. Oligonucleotides for first round of PCR after bisulfite treatment}

\begin{tabular}{|l|l|}
\hline $1^{\text {st }}-$ RBFenhancer-5 & TAAGATTGGAATATTTAGTTTAATTGAA \\
\hline $1^{\text {st }}$-RBFenhancer-3 & CCACCTTCTAATCAACTAAATTCATCTC \\
\hline $1^{\text {st }}-$ RBFexon $1-5$ & GATTAAGTTGTGAAATAGTTATTTTGTT \\
\hline $1^{\text {st }}-$ RBFexon $1-3$ & ACACACAATTACCCTTAACCAC \\
\hline
\end{tabular}




\section{b. Oligonucleotides for second round of PCR after bisulfite treatment}

\begin{tabular}{|l|l|}
\hline $2^{\text {nd }}-$ RBFenhancer-5 & TATAAATTTTATATTTAGAAATGAAATG \\
\hline $2^{\text {nd }}-$ RBFenhancer-3 & TCCACATCCCCCAATTTCC \\
\hline $2^{\text {nd }}-$ RBFexon1-5 & ATTATTTTTGTAATTAAGTGTAAG \\
\hline $2^{\text {nd }}-$ RBFexon1-3 & ACCACCTCAACTCCCAATTC \\
\hline
\end{tabular}

\subsubsection{Oligonucleotides for PCR amplification after digestion with methylation sensitive enzymes}

\begin{tabular}{|l|l|}
\hline MSED-RBFenhancer-5 & CATCTTTTAATGCCCTCTGCGACG \\
\hline MSED-RBFenhancer-3 & ATGACTCTGCCAAGTGACCTCCAG \\
\hline MSED-RBFexon1-5 & CAGAAAAGGGTGTATCGCGAACAC \\
\hline MSED-RBFexon1-3 & CTTTTAGGCAGGCAATGCACAGAA \\
\hline Psq-5 & AATAAACGCTGCCCTGCTTA \\
\hline Psq-3 & TCGGATCAATCTCCATGACA \\
\hline
\end{tabular}

\subsubsection{Plasmids}

\subsubsection{Cloning and expression vectors}

pCR2.1TOPO (Invitrogen)

Standard vector for cloning purposes (www.invitrogen.com)

pBluescript II KS ${ }^{+}$(pBS, Stratagene)

Standard vector for cloning purposes (www.stratagene.com).

pBluescript-FLAG (Maile. 2006)

Derivate of pBluescript II $\mathrm{KS}^{+}$with an insertion of the FLAG-tag sequence upstream of the MCS. This vector was constructed by inserting the annealed LinkerA-oligos (2.1.8.7) into pBluescript using the $\mathrm{Notl} / \mathrm{Xbal}$ restriction sites.

pVL-FLAG (Kwoczynski S. 2002)

Baculovirus-expression vector and derivate of pAcSG2 (Pharmingen) containing an insertion for the sequence of the FLAG-epitope. This vector allows the production of recombinant baculoviruses to express $\mathrm{NH}_{2}$-terminal-FLAG-tagged fusion proteins in SF9cells.

pGEX2TKN (Kwoczynski S. 2002)

Prokaryotic expression vector derived from the pGEX2TK (Amersham Pharmacia Biotech) containing an insertion for the sequence of Clutathion-S-traansferase (GST). This vector can be used for the expression of $\mathrm{NH}_{2}$-terminal-GST-tagged fusion proteins in E. Coli. 
PAcGFP-N1 (Clontech)

Eukaryotic expression vector for the expression of the green fluorescent protein (GFP) from Aequorea coerulescens.

\subsubsection{Cloned constructs}

pBluescript-FLAG-tetR (Kwoczynski S. 2002)

Derivate of pBluescript containing an insertion for the sequence of tetR fragment.

pCR2,1TOPO-MDU (Mora-Bermúdez F. 2002.)

Derivate of $\mathrm{pCR} 2,1 \mathrm{TOPO}$ containing an insertion for the coding sequence of MDU.

pCR2.1TOPO-MDU (H775L)

pCR2,1TOPO derivate with the insertion of MDU PCR fragment. The PCR-fragment was amplified with the MDUmSET-H775L primers (2.1.8.2) following the protocol for inserting point mutations into DNA. Template DNA was pCR2,1TOPO-MDU.

pCR2.1TOPO-MDU-500

pCR2,1TOPO derivate with the insertion of a 500bp PCR fragment of MDU. The PCR

fragment was amplified with the PCRTOPO-MDU500bp primers (2.1.8.3)

Template DNA was pCR2,1TOPO-MDU. The recombinant plasmid was checked by sequencing to select the one that can transcript the anti-sensce strand of MDU under the control of T7 promoter of pCR2.1TOPO.

pCR2.1TOPO-RBF-500

pCR2,1TOPO derivate with the insertion of a 500bp PCR fragment of RBF. The PCR fragment was amplified with the PCRTOPO-RBF500bp primers (2.1.8.3)

Template DNA was RT-PCR product of drosophila embryo. The recombinant plasmid was checked by sequencing to select the one that can transcript the anti-sensce strand of RBF under the control of T7 promoter of pCR2.1TOPO.

pCR2.1TOPO-dDNMT2-500

pCR2,1TOPO derivate with the insertion of a 500bp PCR fragment of dDNMT2. The PCR fragment was amplified with the PCRTOPO-dDNMT500bp primers (2.1.8.3). Template

DNA was RT-PCR product of drosophila embryo. The recombinant plasmid was checked by sequencing to select the one that can transcript the anti-sensce strand of dDNMT2 under the control of T7 promoter of pCR2.1TOPO.

pVLFLAG-MDU

pVLFLAG derivate with the insertion of coding sequence of MDU. The MDU fragment was amplified with the 5'-pVLFLAG-MDU (Nde1) and 3'- pVLFLAG-MDU (BamH1) primers. Template DNA was pCR2,1TOPO-MDU. After digested with Nde1 and BamH1, the fragment was ligated with the pVLFLAG digested with Nde1 and Bglll.

pVLFLAG- $\triangle N M D U$

pVLFLAG derivate with insertion of the $1.9 \mathrm{~kb}$ MDU-PCR-fragment (736-2529). The PCRfragment was amplified with the 5'-pVLFLAG- $\triangle$ NMDU (Nde1) and 3'- pVLFLAG-MDU (BamH1) primers (2.1.8.3.). The template was pCR2.1TOPO-MDU. After digested with Nde1 and BamH1, the fragment was ligated with the pVLFLAG digested with Nde1 and Bglll. 
pVLFLAG- $\triangle$ NMDU (H775L)

pVLFLAG derivate with insertion of the $1.9 \mathrm{~kb}$ MDU (H775L)-PCR-fragment. The PCRfragment was amplified with the 5'-pVLFLAG- $\triangle$ NMDU (Nde1) and 3'- pVLFLAG-MDU (BamH1) primers (2.1.8.3.). The template was pCR2.1TOPO-MDU (H775). After digested with Nde1 and BamH1, the fragment was ligated with the pVLFLAG digested with Nde1 and Bglll.

pVLFLAG-dDNMT2

pVLFLAG derivate with the insertion of coding sequence of dDNMT2. The dDNMT2 fragment was amplified with the 5'-pVLFLAG-dDNMT2 (Nde1) and 3'- pVLFLAGdDNMT2 (Not1) primers (2.1.8.3.). Template DNA was RT-PCR product of drosophila embryo. After digested with Nde1 and Not1, the fragment was ligated with the pVLFLAG digested with Nde1 and Not1.

pGEX2TKN-MBD pGEX2TKN derivate with the insertion of fragment coding for the MBD domain of MDU (1201-1413). The MBD fragment was amplified with the 5'-pGEX-MBD (Nco1) and 3'pGEX-MBD (Xba1) (2.1.8.3.). The template was pCR2.1TOPO-MDU. After digested with Nco1 and Xba1, the fragment was ligated with the pGEX2TKN digested with Nco1 and Xba1.

pGEX2TKN-MBD (R436C) pGEX2TKN derivate with the insertion of fragment coding for the mutated MBD (R436C). PCR-fragment was amplified with the MDUmMBD-R436C primers (2.1.8.2) following the protocol for inserting point mutations into DNA and the template DNA was pGEX2TKNMBD. After digested with Nco1 and Xba1, the PCR fragment coding for mutated MBD domain was ligated with the pGEX2TKN digested with Nco1 and Xba1.

pBluescript-FLAG-MDU

pBluescript-FLAG derivate with an insertion for the coding sequence of MDU. The MDU fragment was amplified with 5'-pBSFLAG-MDU (Nde1) and 3'- pBSFLAG-MDU (Xba1)

(2.1.8.3). After digested with $\mathrm{Nde} 1$ and $\mathrm{Xba1}$, the fragment was ligated with the pBluescript-FLAG digested with Nde1 and Xba1.

pBluescript-FLAG-tetR-MDU pBluescript-FLAG derivate with an insertion coding for the tetR-MDU fusion protein. pBluescript-FLAG-tetR was digested with Nco1 and Nde1. The tetR fragment was recovered after the digestion and liagted with the pBluescript-FLAG-MDU digested with Nco1 and Nde1.

$\mathrm{p} \triangle \mathrm{AcGFP}$-tetR-MDU pAcGFP derivate with an insertion coding for the tetR-MDU fusion protein. The tetR-MDU fragment was amplified with the 5'-pPAc-tetRMDU (BamH1) and 3'- pPAc-tetRMDU (Not1) primers (2.1.8.3). The template was pBluescript-FLAG-tetR-MDU. After digested with BamH1 and Not1, tetR-MDU fragment was ligated with the pAcGFP digested with BamH1 and Not1(thus coding region for GFP is removed from the plamid).

$\mathrm{p} \triangle \mathrm{AcGFP}$-tetR-MDU (H775L)

Eukaryotic expression vector and pAcGFP derivate with an insertion coding for the tetRMDU fusion protein where the MDU is mutated in the SET domain (H775L). Mutation in 
the SET domain of MDU was introduced after $\mathrm{p} \triangle \mathrm{AcGFP}$-tetR-MDU was amplified with the MDUmSET-H775L primers (2.1.8.2) following the protocol for inserting point mutations into DNA. After digesting with BamH1 and Not1, the tetR-MDU (H775L) fragment was ligated with the pAcGFP digested with BamH1 and Not1.

$\mathrm{p} \triangle \mathrm{AcGFP}-\mathrm{MDU}$

Eukaryotic expression vector and pAcGFP derivate with an insertion coding for the MDU protein. The MDU fragment was amplified with the 5'-pPAc-MDU (BamH1) and 3'-pPAcMDU (Not1) (2.1.8.3.) with pCR2,1TOPO-MDU as template. After digested with BamH1 and Not1, the MDU fragment was ligated with the pAcGFP digested with BamH1 and Not1.

$\mathrm{p} \triangle \mathrm{AcGFP}-\mathrm{MDU}(\mathrm{H} 775 \mathrm{~L})$ $\mathrm{p} \triangle \mathrm{AcGFP}-M D U$ derivate where the MDU is mutated in the SET domain ( $\mathrm{H} 775 \mathrm{~L})$. Mutation in the SET domain of MDU was introduced after $\mathrm{p} \triangle \mathrm{AcGFP}$ - MDU was amplified with the MDUmSET-H775L primers (2.1.8.2) following the protocol for inserting point mutations into DNA. After digesting with BamH1 and Not1, the MDU $(\mathrm{H} 775 \mathrm{~L})$ fragment was ligated with the pAcGFP digested with BamH1 and Not1.

$\mathrm{p} \triangle \mathrm{AcGFP}-\mathrm{MDU}(\mathrm{R} 436 \mathrm{C})$ $\mathrm{p} \triangle A c G F P-M D U$ derivate where the MDU is mutated in the MBD domain (R436C). Mutation in the SET domain of MDU was introduced after $\mathrm{p} \triangle \mathrm{AcGFP}$ - MDU was amplified with the MDUmSET-H775L primers (2.1.8.2) following the protocol for inserting point mutations into DNA. After digesting with BamH1 and Not1, the MDU $(\mathrm{H} 775 \mathrm{~L})$ fragment was ligated with the pAcGFP digested with BamH1 and Not1.

\subsubsection{Baculo viruses for expression in Sf9-cellculture}

Baculo viruses were created during this work to express proteins. Methods of constructing baculo-expression vectors is described in chapter

BV means baculo virus.

BV-FLAG-MDU

Baculovirus for expression of the N-terminal FLAG-tagged MDU (CG30426)-fusion protein.

BV-FLAG- $\triangle N M D U$

Baculovirus for expression of the N-terminal FLAG-tagged $\triangle$ NMDU (736-2529) fusion protein.

BV-FLAG- $\triangle$ NMDU (H775L)

Baculovirus for expression of the N-terminal FLAG-tagged SET domain mutated $\triangle$ NMDU fusion protein.

BV-FLAG-dDNMT2

Baculovirus for expression of the N-terminal FLAG-tagged MDU (CG30426)-fusion protein. 


\subsubsection{Media, Buffers and Stock Solutions}

All solutions were prepared in Milli-Q-filtered $\mathrm{dH}_{2} \mathrm{O}$ unless otherwise mentioned below. Solutions which needed to be sterile were autoclaved or sterile-filtered depending on solution type. The $\mathrm{pH}$ of the solutions was adjusted with $\mathrm{HCl}$ or $\mathrm{NaOH}$ unless otherwise mentioned below.

\subsubsection{Media}

LB-media

LB-agar-media

SOB-media:

YEPD-media:

serum-free Sf9-media:

$\begin{array}{ll}1 \% & \text { peptone } \\ 0,5 \% & \text { yeast extract } \\ 0,5 \% & \mathrm{NaCl}\end{array}$

$1.5 \% \quad$ agar in LB-media

$20 \mathrm{~g} \quad$ tryptone

$5 \mathrm{~g} \quad$ yeast extract

$0.5 \mathrm{~g} \quad \mathrm{NaCl}$

$2.5 \mathrm{mM} \quad \mathrm{KCl}$

$\mathrm{pH} 7.0$

add $\mathrm{dH}_{2} \mathrm{O}$ to a final volume of $1 \mathrm{I}$

autoclave

add $5 \mathrm{ml}$ sterile $2 \mathrm{M} \mathrm{MgCl}_{2}$ just before use

$10 \mathrm{~g} \quad$ yeast extract

$20 \mathrm{~g}$ peptone

$960 \mathrm{ml} \quad \mathrm{dH}_{2} \mathrm{O}$

autoclave

add $20 \mathrm{ml}$ sterile glucose solution $(50 \% \mathrm{w} / \mathrm{v})$

1 SF900II serum-free media

1\% Pluronic ${ }^{\circledR}$ F-68

$1 \% \quad$ L-Glutamin/Penicillin/Streptomycin

\subsubsection{Buffers}
AP-buffer:
$100 \mathrm{mM}$
$100 \mathrm{mM}$
Tris- $\mathrm{HCl}, \mathrm{pH} 8.0$
$50 \mathrm{mM}$
$\mathrm{NaCl}$
$\mathrm{MgCl}_{2}$
10x PAGE-buffer:

$\begin{array}{ll}2 \mathrm{M} & \text { Glycine } \\ 250 \mathrm{mM} & \text { Tris-HCl pH } 8.3\end{array}$




\begin{tabular}{|c|c|c|}
\hline SDS-PAGE-running buffer: & $\begin{array}{l}4.5 \mathrm{l} \\
500 \mathrm{ml} \\
25 \mathrm{ml}\end{array}$ & $\begin{array}{l}\mathrm{dH}_{2} \mathrm{O} \\
10 x \text { PAGE-buffer } \\
20 \% \text { SDS }\end{array}$ \\
\hline $\begin{array}{l}\text { Transfer buffer l: } \\
\text { (proteins < } 150 \text { kDa) }\end{array}$ & $\begin{array}{l}3.5 \mathrm{l} \\
1 \mathrm{l} \\
500 \mathrm{ml} \\
2.5 \mathrm{ml}\end{array}$ & $\begin{array}{l}\mathrm{dH}_{2} \mathrm{O} \\
\text { Methanol } \\
10 x \text { PAGE-buffer } \\
20 \% \text { SDS }\end{array}$ \\
\hline $\begin{array}{l}\text { Transfer buffer II: } \\
\text { (proteins >150 kDa) }\end{array}$ & $\begin{array}{l}50 \mathrm{mM} \\
380 \mathrm{mM} \\
0.1 \% \\
20 \%\end{array}$ & $\begin{array}{l}\text { Tris-HCl pH } 8.3 \\
\text { Glycine } \\
\text { SDS (w/v) } \\
\text { Methanol }\end{array}$ \\
\hline 4x SDS-loading buffer: & $\begin{array}{l}125 \mathrm{mM} \\
10 \% \\
6 \% \\
20 \% \\
1 \text { grain/10ml }\end{array}$ & $\begin{array}{l}\text { Tris-HCl, pH } 6.8 \\
\beta \text {-Mercaptoethanol } \\
\text { SDS } \\
\text { Glycerol } \\
\text { Bromophenolblue }\end{array}$ \\
\hline $\begin{array}{l}\text { TBjap-buffer: } \\
\text { (Transformation buffer Japanese) }\end{array}$ & $\begin{array}{l}10 \mathrm{mM} \\
15 \mathrm{mM} \\
250 \mathrm{mM} \\
\text { set } \mathrm{pH} \text { to } 6.7 \\
55 \mathrm{mM} \\
\text { filter-sterilizat }\end{array}$ & $\begin{array}{l}\text { Pipes } \\
\mathrm{CaCl}_{2} \\
\mathrm{KCl} \\
\mathrm{KOH}) \\
\mathrm{MnCl}_{2} \\
\text { on }\end{array}$ \\
\hline 5x TBE-buffer: & $\begin{array}{l}54 \mathrm{~g} \\
27.5 \mathrm{~g} \\
20 \mathrm{ml} \\
\text { add } \mathrm{dH}_{2} \mathrm{O}(\mathrm{Mi}\end{array}$ & $\begin{array}{l}\text { Tris- } \mathrm{HCl}, \mathrm{pH} 8.0 \\
\text { Boric acid } \\
0.5 \mathrm{M} \text { EDTA } \\
\text { li-Q) to a total volume of } 1 \text { I }\end{array}$ \\
\hline TBST-buffer: & $\begin{array}{l}100 \mathrm{mM} \\
150 \mathrm{mM} \\
0.1 \%\end{array}$ & $\begin{array}{l}\text { Tris-HCl, pH } 8.0 \\
\text { NaCl } \\
\text { Tween }{ }^{\circledR} 20\end{array}$ \\
\hline TE-buffer: & $\begin{array}{l}10 \mathrm{mM} \\
1 \mathrm{mM}\end{array}$ & $\begin{array}{l}\text { Tris-HCl, pH } 8.0 \\
\text { EDTA }\end{array}$ \\
\hline $\begin{array}{l}\text { Plasmid miniprep-lysis buffer: } \\
\text { (LiCl-method) }\end{array}$ & $\begin{array}{l}7.8 \mathrm{ml} \\
1.3 \mathrm{ml} \\
0.4 \mathrm{ml} \\
0.25 \mathrm{ml} \\
0.25 \mathrm{ml} \\
250 \mathrm{\mu l}\end{array}$ & $\begin{array}{l}\text { 3.2 M LiCl } \\
0.5 \mathrm{M} \text { EDTA, pH } 8.0 \\
10 \% \text { Triton X } 100 \\
2 \mathrm{M} \text { Tris pH } 7.5 \\
\mathrm{dH}_{2} \mathrm{O} \\
\text { Lysozyme stock solution }\end{array}$ \\
\hline Yeast lysis buffer: & $\begin{array}{l}10 \mathrm{mM} \\
100 \mathrm{mM} \\
1 \% \\
2 \% \\
1 \mathrm{mM}\end{array}$ & $\begin{array}{l}\text { Tris-HCl, pH } 8.0 \\
\text { NaCl } \\
\text { SDS } \\
\text { Triton X-100 } \\
\text { EDTA }\end{array}$ \\
\hline
\end{tabular}




\begin{tabular}{|c|c|c|}
\hline DNA-loading buffer 10x: & $\begin{array}{l}50 \% \\
0.1 \% \\
49.9 \%\end{array}$ & $\begin{array}{l}\text { Glycerol } \\
\text { Orange-G } \\
\text { 1x TBE-buffer }\end{array}$ \\
\hline EMSA gel loading buffer 10x: & $\begin{array}{l}50 \% \\
0.25 \% \\
0.25 \%\end{array}$ & $\begin{array}{l}\text { Glycerol } \\
\text { Xylene cyanol } \\
\text { Bromophenol blue }\end{array}$ \\
\hline 5x HEMG-buffer: & $\begin{array}{l}125 \mathrm{mM} \\
62.5 \mathrm{mM} \\
0.5 \mathrm{mM} \\
50 \%\end{array}$ & $\begin{array}{l}\text { Hepes, pH } 7.6 \\
\mathrm{MgCl}_{2} \\
\text { EDTA } \\
\text { Glycerol }\end{array}$ \\
\hline 0.5 M NaCl-1x HEMG-buffer: & $\begin{array}{l}0.5 \mathrm{M} \\
1 \% \\
20 \% \\
1 \mathrm{mM} \\
10 \mu \mathrm{g} / \mathrm{ml} \\
10 \mu \mathrm{g} / \mathrm{ml}\end{array}$ & $\begin{array}{l}\mathrm{NaCl} \\
\mathrm{NONIDET}^{\circledR} \text { P40 } \\
5 x \text { HEMG-buffer } \\
\text { PMSF (freshly added) } \\
\text { leupeptin (freshly added) } \\
\text { pepstatin (freshly added) }\end{array}$ \\
\hline 5x NEGN-buffer: & $\begin{array}{l}100 \mathrm{mM} \\
5 \mathrm{mM} \\
50 \%(\mathrm{v} / \mathrm{v})\end{array}$ & $\begin{array}{l}\text { Tris, pH } 8.0 \\
\text { EDTA } \\
\text { Glycerol }\end{array}$ \\
\hline 0.5 M NaCl-1x NEGN-buffer: & $\begin{array}{l}0.5 \mathrm{M} \\
20 \mathrm{mM} \\
1 \mathrm{mM} \\
10 \%(\mathrm{v} / \mathrm{v}) \\
1 \%(\mathrm{v} / \mathrm{v}) \\
1 \mathrm{mM} \\
10 \mu \mathrm{g} / \mathrm{ml} \\
10 \mu \mathrm{g} / \mathrm{ml}\end{array}$ & $\begin{array}{l}\mathrm{NaCl} \\
\text { Tris, pH } 8.0 \\
\text { EDTA } \\
\text { Glycerol } \\
\text { NONIDET }^{\circledR} \text { P40 } \\
\text { PMSF (freshly added) } \\
\text { leupeptin (freshly added) } \\
\text { pepstatin (freshly added) }\end{array}$ \\
\hline $5 x$ Binding buffer: & $\begin{array}{l}125 \mathrm{mM} \\
250 \mathrm{mM} \\
5 \mathrm{mM} \\
25 \%(\mathrm{v} / \mathrm{v})\end{array}$ & $\begin{array}{l}\text { Tris- HCl, pH } 7.5 \\
\text { KCl } \\
\text { DTT } \\
\text { Glycerol }\end{array}$ \\
\hline 1x Binding buffer: & $\begin{array}{l}25 \mathrm{mM} \\
50 \mathrm{mM} \\
1 \mathrm{mM} \\
5 \%(\mathrm{v} / \mathrm{v})\end{array}$ & $\begin{array}{l}\text { Tris- HCl, pH } 7.5 \\
\mathrm{KCl} \\
\text { DTT } \\
\text { Glycerol }\end{array}$ \\
\hline $0.5 \mathrm{M} \mathrm{NaCl}-1 \mathrm{x}$ binding buffer: & $\begin{array}{l}0.5 \mathrm{M} \\
25 \mathrm{mM} \\
50 \mathrm{mM} \\
1 \mathrm{mM} \\
5 \%(\mathrm{v} / \mathrm{v})\end{array}$ & $\begin{array}{l}\mathrm{NaCl} \\
\text { Tris- } \mathrm{HCl}, \mathrm{pH} 7.5 \\
\mathrm{KCl} \\
\text { DTT } \\
\text { Glycerol }\end{array}$ \\
\hline
\end{tabular}


20x Oligo annealing buffer:

$\begin{array}{ll}200 \mathrm{mM} & \text { Tris- } \mathrm{HCl}, \mathrm{pH} 7.9 \\ 40 \mathrm{mM} & \mathrm{MgCl}_{2} \\ 1 \mathrm{M} & \mathrm{NaCl} \\ 20 \mathrm{mM} & \text { EDTA }\end{array}$

10x PBS:

$\begin{array}{ll}1.4 \mathrm{M} & \mathrm{NaCl} \\ 27 \mathrm{mM} & \mathrm{KCl} \\ 100 \mathrm{mM} & \mathrm{Na}_{2} \mathrm{HPO}_{4} \\ 18 \mathrm{mM} & \mathrm{KH}_{2} \mathrm{PO}_{4} \\ \text { pH } 7.3 & \end{array}$

IP-lysis buffer:

$\begin{array}{ll}25 \mathrm{mM} & \text { Tris, pH } 7.5 \\ 140 \mathrm{mM} & \text { NaCl } \\ 1 \mathrm{mM} & \text { EDTA } \\ 1 \% & \text { Triton-X-100 } \\ 0.1 \% & \text { SDS } \\ \text { filter-sterilization } \\ 1 \mathrm{mM} & \text { PMSF (freshly added) } \\ 10 \mu \mathrm{g} / \mathrm{ml} & \text { leupeptin (freshly added) } \\ 10 \mu \mathrm{g} / \mathrm{ml} & \text { pepstatin (freshly added) }\end{array}$

IP1:

similar as XChIP-lysisbuffer but with $500 \mathrm{mM}$ $\mathrm{NaCl}$ instead

IP2:

$\begin{array}{ll}10 \mathrm{mM} & \text { Tris, } \mathrm{pH} 8.0 \\ 250 \mathrm{mM} & \text { LiCl } \\ 0.5 \% & \text { NONIDET }^{\circledR} \mathrm{P}^{2} 0 \\ 0.5 \% & \text { Sodiumdesoxycholate } \\ 1 \mathrm{mM} & \text { EDTA } \\ \text { filter-sterilization }\end{array}$

Wash buffer:

$\begin{array}{ll}10 \mathrm{mM} & \text { Hepes pH 7.6 } \\ 200 \mathrm{mM} & \mathrm{NaCl} \\ 1 \mathrm{mM} & \text { EDTA } \\ 0.5 \mathrm{mM} & \text { EGTA } \\ 0.01 \% & \text { Triton-X-100 }\end{array}$

Binding buffer:

$\begin{array}{ll}50 \mathrm{mM} & \text { Hepes pH 7.6 } \\ 100 \mathrm{mM} & \mathrm{NaCl} \\ 1 \mathrm{mM} & \text { EDTA } \\ 0.5 \mathrm{mM} & \text { EGTA }\end{array}$

Methyltransferase buffer:

$\begin{array}{ll}25 \mathrm{mM} & \text { Tris, pH } 8.0 \\ 100 \mathrm{mM} & \mathrm{NaCl} \\ 1 \mathrm{mM} & \text { DTT } \\ 1 \mathrm{mM} & \text { PMSF }\end{array}$

Nuclear Buffer 1:

$\begin{array}{ll}15 \mathrm{mM} & \text { Hepes, } \mathrm{pH} 7.8 \\ 10 \mathrm{mM} & \mathrm{KCl} \\ 5 \mathrm{mM} & \mathrm{MgCl}_{2} \\ 0.1 \mathrm{mM} & \text { EDTA }\end{array}$




$\begin{array}{ll}0.5 \mathrm{mM} & \text { EGTA } \\ 350 \mathrm{mM} & \text { Sucrose } \\ 1 \mathrm{mM} & \text { DTT } \\ 1 \mathrm{mM} & \text { Sodium Metabisulfite } \\ 1 \mathrm{mM} & \text { PMSF (freshly added) } \\ 10 \mu \mathrm{g} / \mathrm{ml} & \text { leupeptin (freshly added) } \\ 10 \mu \mathrm{g} / \mathrm{ml} & \text { pepstatin (freshly added) }\end{array}$

\subsubsection{General stock solutions}

\begin{tabular}{|c|c|c|}
\hline Ethidiumbromide solution: & $10 \mathrm{mg} / \mathrm{ml}$ & Ethidiumbromide in $\mathrm{dH}_{2} \mathrm{O}$ \\
\hline Ampicillin stock solution: & $100 \mathrm{mg} / \mathrm{ml}$ & Ampicillin (Na-salt) \\
\hline PMSF-stock solution: & $200 \mathrm{mM}$ & PMSF in isopropanol \\
\hline Lysozyme stock solution: & $10 \mathrm{mg} / \mathrm{ml}$ & Lysozyme in $\mathrm{dH}_{2} \mathrm{O}$ \\
\hline Proteinase-K solution: & $20 \mathrm{mg} / \mathrm{ml}$ & Proteinase $\mathrm{K}$ in $\mathrm{dH}_{2} \mathrm{O}$ \\
\hline NBT-solution: & $\begin{array}{l}0.5 \mathrm{~g} \\
10 \mathrm{ml}\end{array}$ & $\begin{array}{l}\text { Nitro-blue-tetrazolium-chloride } \\
70 \% \text { dimethylformamide }\left(\mathrm{dH}_{2} \mathrm{O}\right)\end{array}$ \\
\hline BCIP-solution: & $\begin{array}{l}0.5 \mathrm{~g} \\
10 \mathrm{ml}\end{array}$ & $\begin{array}{l}\text { 5bromo-4chloro-3-indolylphosphate } \\
\text { (disodium salt) } \\
100 \% \text { dimethylformamide }\end{array}$ \\
\hline IPTG solution: & $1 \mathrm{M}$ & $\begin{array}{l}\text { Isopropyl-- } \beta \text {-D- } \\
\text { thiogalactopyranoside in } \mathrm{dH}_{2} \mathrm{O}\end{array}$ \\
\hline X-gal solution & $40 \mathrm{mg} / \mathrm{ml}$ & $\begin{array}{l}\text { 5-bromo-4-chloro- } \\
\text { 3indolyl- } \beta \text {-D-galactoside in DMF }\end{array}$ \\
\hline
\end{tabular}

\subsubsection{Protein and nondenaturing polyacrylamide gel solutions}

$\begin{array}{lll}\text { Coomassie-destaining-solution: } & 10 \% & \text { Acetic acid } \\ & 45 \% & \text { Methanol } \\ & 45 \% & \mathrm{dH}_{2} \mathrm{O} \\ & & \\ \text { Coomassie-staining-solution: } & 0.25 \% & \text { Coomassie }{ }^{\circledR} \mathrm{R} 250 \text { Brilliant Blue } \\ & 9.75 \% & \text { Acetic acid } \\ & 45 \% & \text { Methanol } \\ & 45 \% & \mathrm{dH}_{2} \mathrm{O}\end{array}$




\begin{tabular}{|c|c|c|}
\hline $\begin{array}{l}\text { SDS-PAGE-resolving-gel: } \\
\text { (in } \mathrm{dH}_{2} \mathrm{O} \text { ) }\end{array}$ & $\begin{array}{l}25 \% \\
6-18 \% \\
0.1 \% \\
0.6 \%\end{array}$ & $\begin{array}{l}\text { 4x resolving-gel buffer: } \\
\text { Acrylamide, } 37.5: 1 \text { (depending on } \\
\text { gel concentration) } \\
\text { TEMED } \\
\text { APS }\end{array}$ \\
\hline $\begin{array}{l}\text { SDS-PAGE-stacking-gel: } \\
\text { (in } \mathrm{dH}_{2} \mathrm{O} \text { ) }\end{array}$ & $\begin{array}{l}25 \% \\
4 \% \\
0.1 \% \\
0.6 \%\end{array}$ & $\begin{array}{l}\text { 4x stacking-gel buffer } \\
\text { Acrylamide, 37.5:1 } \\
\text { TEMED } \\
\text { APS }\end{array}$ \\
\hline $\begin{array}{l}4 \mathrm{x} \text { resolving-gel buffer: } \\
\text { (lower tris buffer) }\end{array}$ & $\begin{array}{l}1.5 \mathrm{M} \\
0.4 \%\end{array}$ & $\begin{array}{l}\text { Tris-HCl pH } 8.8 \\
\text { SDS }\end{array}$ \\
\hline $\begin{array}{l}4 x \text { stacking-gel buffer: } \\
\text { (upper tris buffer) }\end{array}$ & $\begin{array}{l}0.5 \mathrm{M} \\
0.4 \%\end{array}$ & $\begin{array}{l}\text { Tris-HCl pH } 6.8 \\
\text { SDS }\end{array}$ \\
\hline $\begin{array}{l}\text { Nondenaturing polyacrylamide gel } \\
\text { (in } \mathrm{dH}_{2} \mathrm{O} \text { ) }\end{array}$ & $\begin{array}{l}0.5 x \\
6-8 \% \\
0.1 \% \\
0.6 \%\end{array}$ & $\begin{array}{l}\text { TBE } \\
\text { Acrylamide, } 29: 1 \text { depending on } \\
\text { gel concentration) } \\
\text { TEMED } \\
\text { APS }\end{array}$ \\
\hline
\end{tabular}

\subsubsection{Silver staining-solutions}

Fixative 1:

Fixative 2:

Silvernitrate-solution:

Developer-solution:

Stop-solution:
$400 \mathrm{ml}$
Ethanol
$100 \mathrm{ml}$
Acetic acid
add $\mathrm{dH}_{2} \mathrm{O}$ (Milli-Q) to a total volume of 1 I

$\begin{array}{ll}300 \mathrm{ml} & \text { Ethanol } \\ 2.5 \mathrm{~g} & \text { Potassium tetrathionate } \\ 41 \mathrm{~g} & \text { Sodium acetate, anhydrous } \\ \text { add } \mathrm{dH}_{2} \mathrm{O} \text { (Milli-Q) to a total volume of 1 I }\end{array}$

$2 \mathrm{~g}$

$\mathrm{AgNO}_{3}$

add $\mathrm{dH}_{2} \mathrm{O}$ (Milli-Q) to a total volume of 1 I

add $250 \mu \mathrm{l} 37 \%$ formaldehyde just before use

$\begin{array}{ll}15 \mathrm{~g} & \text { Potassium carbonate } \\ 7.5 \mathrm{mg} & \text { Sodium thiosulfate }\end{array}$

add $\mathrm{dH}_{2} \mathrm{O}$ (Milli-Q) to a total volume of $1 \mathrm{I}$

add $150 \mu \mathrm{l} 37 \%$ formaldehyde just before use

$\begin{array}{ll}50 \mathrm{~g} & \text { Tris base } \\ 20 \mathrm{ml} & \text { Acetic acid } \\ \text { add } \mathrm{dH}_{2} \mathrm{O} & (\text { Milli-Q) to a total volume of 1 I }\end{array}$ 


\subsubsection{Drosophila Stocks}

Wild type Drosophila melanogaster: OregonR (Bloomington stock centre)

12196-48 (Stabell et al., 2006): Transgenic flies generated by P-element mediated transformation. A double stranded (ds) RNA construct UAS-dEset.IR which contains a $540 \mathrm{bp}$ fragment of $M d u$ (CG30426) cDNA was integrated into the chromosome 2. This strain is a gift from Andrew Lambertsson (Institute of Molecular Biosciences, University of Oslo, Norway).

6313 (Nichols et al., 1996): Transgenic flies. Genotype is P\{GawB\}lz[gal4] for GAL4 expression in eye disc. This strain is bought from Bloomington stock center. 


\subsection{Methods}

\subsubsection{Analysis and Manipulation of Nucleic Acids}

\subsubsection{Photometric determination of DNA/RNA concentrations}

Nucleic acids absorb light in the ultraviolet range. The absorption-maximum of DNA is at 260 $\mathrm{nm}, \mathrm{RNA}$ and proteins (aromatic residues) at $280 \mathrm{~nm}$ respectively. To determine the concentration of DNA, the sample was tested for absorption at $260 \mathrm{~nm}$ in a spectrophotometer (2.1.1). To determine the degree of impurities due to RNA and proteins the absorption (optical density or OD) at $280 \mathrm{~nm}$ was also measured. The quotient of $O D_{260} / O D_{280}$ is an indicator for the purity of DNA and lies within 1.8 to 2.0 for pure (column purification) solutions. For calculation of the DNA-concentration, following conventional unit was used: an $\mathrm{OD}_{260}$ of 1 resembles a concentration of $50 \mu \mathrm{g}$ double-stranded DNA per $\mathrm{ml}$.

\subsubsection{Polymerase chain reaction (PCR)}

\subsection{PCR for cloning of cDNA-fragments}

To amplify cDNAs out of template DNA-pools (Plasmidpreps, Libraries), primers containing specific restriction enzyme cutting sites were created (2.1.8.3). The PCR reaction used 50-100 ng DNA template, 1-2 U DeepVent proofreading polymerase (NEB), $2 \mu$ l of 10x ThermoPol reaction buffer (NEB), $1 \mu \mathrm{l}$ of $2.5 \mathrm{mM}$ dNTP-Mix (TaKaRa) and $1 \mu \mathrm{M}$ endconcentration of the corresponding 5'- and 3'-Primer. The reaction was set to a total volume of $20 \mu \mathrm{l}\left(\mathrm{ad} \mathrm{dH}_{2} \mathrm{O}\right)$. After initial denaturation of $95^{\circ} \mathrm{C}$ for $1.5 \mathrm{~min}$. around 25 to 30 amplification cycles followed: melting temperature was set to $95^{\circ} \mathrm{C}\left(30 \mathrm{sec}\right.$.), annealing around $5^{\circ} \mathrm{C}$ below the average melting temperature of the specific primerpair (45 sec.) and elongation was set to $75^{\circ} \mathrm{C}(1 \mathrm{~min}$. per $1 \mathrm{~kb}$ fragment length). The PCR ended with a final elongation at $75^{\circ} \mathrm{C}$ for $20 \mathrm{~min}$. The amplified DNA was analyzed and purified afterwards via electrophoresis on an agarose gel (2.2.1.6).

\subsection{PCR for standard detection of specific DNA-sequences}

To detect primer specific DNA-sequences in a sample by amplification, a PCR-reaction was set

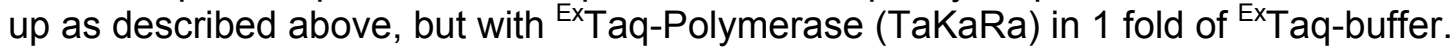
Denaturation temperature was set to $95^{\circ} \mathrm{C}$, elongation to $72^{\circ} \mathrm{C}$. 


\subsection{Reverse-Transcriptase-PCR (RT-PCR)}

RNA, isolated with the midiprep kit from Qiagen, was measured on a spectrophotometer (Biorad) and used in a concentration of $10 \mu \mathrm{g}$ per reaction. The RNA has been incubated at $65^{\circ} \mathrm{C}$ for 5 min and subsequently cooled down on ice. For the reverse transcriptase (RT) reaction $20 \mu$ of 5x RT-buffer (Invitrogen), $10 \mu \mathrm{l} 0.1 \mathrm{M}$ DTT, $10 \mu \mathrm{l}$ random hexamer primers (Boehringer Mannheim [200 ng/ $\mu \mathrm{l}]), 10 \mu \mathrm{l}$ of $10 \mathrm{mM}$ dNTPs, $2.5 \mu \mathrm{l}$ RNasin and $2 \mu \mathrm{l}$ Superscript RT were added and the sample was set to a total volume of $100 \mu \mathrm{l}\left(\mathrm{dH}_{2} \mathrm{O}\right)$. After incubation at $37^{\circ} \mathrm{C}$ for 1 $\mathrm{h}$. the sample was heated up to $95^{\circ} \mathrm{C}$ for $5 \mathrm{~min}$ and then cooled down on ice. The cDNA produced with this reaction could be stored at $-20^{\circ} \mathrm{C}$ or immediately analyzed via PCR (2.2.1.2.2).

\subsubsection{Radiolabeling of single strand Oligo DNA}

To label the single strand DNA oligo (2.1.8.1) at the 5 ' end with ${ }^{32} \mathrm{P}, 1 \mu \mathrm{l}$ of $10 \mathrm{pM}$ oligo DNA, $1 \mu \mathrm{l}$ of $10 x$ phosphorylation buffer, $3 \mu \mathrm{l}$ of $10 \mathrm{mCi} / \mathrm{ml}\left[\gamma_{-}{ }^{32} \mathrm{P}\right]-\mathrm{dATP}, 1 \mu \mathrm{l}$ of $5 \mathrm{U} / \mu \mathrm{l} \mathrm{T} 4$ polynucleotide kinase and $4 \mu \mathrm{l}$ of water were mix and incubated at $37^{\circ} \mathrm{C}$ for $1 \mathrm{~h}$ and $30 \mathrm{~min}$. The labeled DNA was used in the following annealing reaction.

\subsubsection{Annealing of single-strand DNA}

To create double-strand DNA with ${ }^{32} \mathrm{P}$ labeled single strand DNA oligos and their complimentary strands, $10 \mu \mathrm{l}$ of labeled oligo DNA (2.2.1.3) was mixed with $3 \mu \mathrm{l} 10 \mathrm{pM}$ complimentary DNA oligo, $2 \mu \mathrm{l}$ of $20 x$ oligo annealing buffer (2.1.11.2), and $25 \mu \mathrm{l}$ water. The mixture was heated up to $95^{\circ} \mathrm{C}$ for $5 \mathrm{~min}$ and then cooled down slowly to room temperature. Annealed radioactive double strand DNA was purified with Qiaquick Nucleotide Removal Kit (2.1.2) and then stored at $4{ }^{\circ} \mathrm{C}$ for the DNA-precipitation assay.

\subsubsection{Digestion of DNA with restriction endonucleases}

The digestion of DNA with restriction endonucleases was performed in a standard volume of 20 $\mu \mathrm{l}$ with sticky-end or blunt-end cutting restriction enzymes of bacterial origin. Single and double digest temperature, usage of BSA as a stabilizing agent and buffer settings were chosen following the recommendations of the manufacturer (NEB). 2-5 $U$ (units) of enzyme were used for $2 \mathrm{~h}$ digestion assays, overnight digests were performed using $5 \mathrm{U}$ of enzyme. Digested DNA was either dephosphorylated (2.2.1.6) or directly analyzed and purified via gel electrophoresis (2.2.1.7). 


\subsubsection{Dephosphorylation of linearized plasmids}

To prevent intramolecular religation with the 3'-hydroxy-ends of digested linearised vector-DNA in following ligation reactions, the free 5'-phosphate-groups of the vectors were removed using shrimp alkaline phosphatase (SAP, Boehringer Mannheim) directly after digestion. $2.3 \mu \mathrm{l}$ of 10x dephosphorylation buffer and $1 \mathrm{U}$ of shrimp alkaline phosphatase were added to $20 \mu$ digestion sample and incubated at $37^{\circ} \mathrm{C}$ for $1 \mathrm{~h}$. The sample was then purified from contaminants using gel electrophoresis (2.2.1.7).

\subsubsection{Agarose gel electrophoresis}

Depending on the size of the expected DNA-fragments, gels were used within concentrations of $0.7-2 \%$ agarose. The specific amount of agarose was solubilised in $1 \times$ TBE by melting in a microwave and poured into a gel tray (custom DNA-gel system, 2.1.1) after addition of ethidiumbromide-solution $(0.1 \mu \mathrm{l} / \mathrm{ml}$ of gel). 10x DNA-loading buffer has been added to the DNA samples in 1/10 concentration and the samples were separated by electrophoresis. $0.1-1 \mu \mathrm{g}$ DNA in 20-25 $\mu \mathrm{l}$ sample volume was separated, "Gene-Ruler TM 1 kb DNA ladder" (MBI) was used as DNA marker $(0.1-1 \mu \mathrm{g})$. The gel was then analyzed and documented using the "Eagle Eye" UV-system (Stratagene).

\subsubsection{Isolation of DNA-fragments out of agarose gels}

DNA was extracted of agarose gels using the Perfectprep Gel Cleanup Kit from Eppendorf (2.1.2). The fragment of interest was cut out of the gel, melted in a binding buffer and then bound to the column matrix by means of centrifugation. Following a washing step, the purified DNA can be eluted using water or buffer.

\subsubsection{Ligation of DNA-fragments}

Ligation of DNA-fragments was done using the Quick Ligation Kit from NEB. Dephosphorylated vector-DNA and insert-DNA were combined in a volume of $10 \mu \mathrm{l}$, containing at least five molar excess of insert. $10 \mu \mathrm{l}$ of $2 x$ ligation buffer (NEB) and $1 \mu \mathrm{l}$ of Quick T4 DNA ligase were added, the sample was incubated for $5 \mathrm{~min}$. at $25^{\circ} \mathrm{C}$ and then cooled down on ice. The ligated DNA was directly used to transform competent E.coli cells.

\subsubsection{Growing transformation-competent E.coli cells}

The establishment of transformation-competent cells followed a modified method of Stratagene's Epicurian competent XL-1 blue (Inoue et al. 1990): E. coli XL-1 blue cells (2.1.6) were grown over night in $5 \mathrm{ml} \mathrm{SOB}$ media. The culture was transferred afterwards into $250 \mathrm{ml} \mathrm{SOB}$ media and grown at $18^{\circ} \mathrm{C}$ for approximately $31 \mathrm{~h}$ until it reached an $\mathrm{OD}_{600}$ of 0.6 . It was then incubated on ice for $10 \mathrm{~min}$ and centrifuged in a pre-cooled Sorvall GS3 rotor for 10 min with $2500 \mathrm{~g}$ at $4^{\circ} \mathrm{C}$. Meanwhile $2 \mathrm{ml}$ of $100 \%$ DMSO was added to $100 \mathrm{ml}$ of TBjap (2.1.12.2). After centrifugation, the supernatant has been discarded and the cell 
pellet has been resuspended in $80 \mathrm{ml}$ TBjap buffer with DMSO. The suspension was incubated on ice for $10 \mathrm{~min}$ and centrifuged again. The supernatant has been discarded and the pellet was resuspended in $18.6 \mathrm{ml}$ of precooled TBjap (without DMSO). After adding $1.4 \mathrm{ml}$ of DMSO to the suspension (final concentration of 7\%), it was incubated again on ice for $10 \mathrm{~min}$. After incubation the cell suspension was dispensed into $200 \mu \mathrm{l}$ aliquots in cryotubes which were frozen immediately in liquid nitrogen and were stored at $-80^{\circ} \mathrm{C}$.

\subsubsection{Transformation of competent E.coli cells}

Frozen competent XL-1 blue cells were slowly thawed on ice. Pre-chilled DNA sample (25 ng ligation-mixture-DNA or $100 \mathrm{ng}$ plasmid-DNA) was added to $100 \mu \mathrm{l}$ of cells, the sample was mixed gently and incubated on ice for $30 \mathrm{~min}$. After the following heat shock of $37^{\circ} \mathrm{C}$ for $1.5-2$ min, the sample was chilled on ice again for $5 \mathrm{~min} .900 \mu \mathrm{l}$ of SOB or LB media was added and the solution was incubated at $37^{\circ} \mathrm{C}$ for $1 \mathrm{~h}$. $100 \mu \mathrm{l}$ were spread on a 1:10 dilution LB-agar-plate containing $0.1 \mathrm{mg} / \mathrm{ml}$ ampicillin. The remaining $900 \mu \mathrm{l}$ were centrifuged, the cell pellet was resuspended in $100 \mu \mathrm{l}$ media and spread on a 1:1 plate. The plates were incubated at $37^{\circ} \mathrm{C}$ over night and checked for colonies the next day. Single colonies were picked with a pipet tip, transferred into a bacteria-culture vial with $5 \mathrm{ml}$ LB-media containing $0.1 \mathrm{mg} / \mathrm{ml}$ ampicillin and were grown over night in an incubator-shaker.

\subsubsection{Isolation of plasmid-DNA out of E.coli cells}

\subsection{Plasmid isolation with LiCl}

$1.5 \mathrm{ml}$ out of the $5 \mathrm{ml}$ overnight culture (2.2.1.10) were centrifuged ( $5 \mathrm{~min}, 1000 \mathrm{~g}$, Eppendorf centrifuge) and the resulting cell pellet was resuspended in $200 \mu$ l plasmid prep-lysis buffer (2.1.11.2). After incubation for $5 \mathrm{~min}$ at room temperature, the sample was boiled at $100^{\circ} \mathrm{C}$ for 90 sec and immediately cooled on ice. Chromosomal DNA and denatured proteins were separated from the plasmid-DNA by centrifugation at $16000 \mathrm{~g}$ for $8 \mathrm{~min}$ and the formed pellet was removed from the plasmid containing supernatant using a pipet tip. The plasmid-DNA was precipitated then by adding 0.7 volumes of isopropanol, incubated at room temperature for $10 \mathrm{~min}$ and centrifuged at $16000 \mathrm{~g}$ for $15 \mathrm{~min}$. The supernatant was discarded, the DNA-pellet washed with $70 \%$ ethanol and centrifuged again at $16000 \mathrm{~g}$ for $5 \mathrm{~min}$. The supernatant was pipetted off carefully and the pellet was dried in the speedvac concentrator. After the sample was dry, the

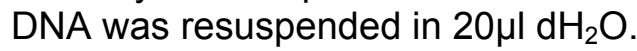

\subsection{Plasmid isolation with the QIAGEN Miniprep kit}

To isolate plasmid-DNA with additional purification through a mini size column, the Qiagen miniprep kit was used. The isolation was performed following the protocol of the manufacturer. For each column DNA of $3 \mathrm{ml}$ of the $5 \mathrm{ml}$ overnight culture was used. The DNA has always been eluated from the column using $30 \mu$ l eluation buffer of the manufacturer. 


\subsection{Plasmid isolation with the QIAGEN Maxiprep kit}

To isolate plasmid-DNA with additional purification through a maxi size column, Qiagens maxiprep kit was used. The $5 \mathrm{ml}$ overnight culture was inoculated into a $250 \mathrm{ml}$ LB-culture and again grown over night. The isolation was performed according to the protocol of the manufacturer, the plasmid-DNA was eluated in 100-500 $\mu$ l TE-buffer.

\subsubsection{Isolation the yeast genomic DNA}

To isolate the genomic DNA of yeast, a method called "smash and grab" was adopted. $50 \mathrm{ml}$ of overnight yeast culture was centrifuged at $3000 \mathrm{rpm}$ for $10 \mathrm{~min}$ at $4^{\circ} \mathrm{C}$. The yeast in pellet was resuspended with $4 \mathrm{ml}$ yeast lysis buffer (2.1.11.2). $6 \mathrm{~g}$ glass beads (2.1.2) and $4 \mathrm{ml} 1: 1$ phenol;chloroform was added to the resuspended yeast and the mixture was vortexed for $2 \mathrm{~min}$. Then $4 \mathrm{ml} \mathrm{TE}$ with $1 \mathrm{mM}$ EDTA was added. Again it was vortexed for $30 \mathrm{sec}$ and then centrifuged at $15,000 \mathrm{rpm}$ for $5 \mathrm{~min}$ at $4^{\circ} \mathrm{C}$. The supernatant was transferred to another centrifuge tube and 1 volume of chloroform was added to it. The mixture was vortexed $30 \mathrm{sec}$ and centrifuged at $4000 \mathrm{rpm}$ for $10 \mathrm{~min}$ at $4{ }^{\circ} \mathrm{C}$. The supernatant from this step was extracted with chloroform again like just mentioned. Then RNase $A$ was added to a final concentration of $50 \mu \mathrm{g} / \mathrm{ml}$. The crude extract was incubated at $37^{\circ} \mathrm{C}$ for 30 min to remove the RNA. After that, 1 volume of phenol was added to extract protein from the DNA like just did with chloroform. This was followed by a similar extraction with chloroform. 2 volumes of ethanol and 0.1 volume of $\mathrm{NaAc}$ were added to the supernatant from last step. Then it was well mixed and centrifuged at $15000 \mathrm{rpm}$ for $15 \mathrm{~min}$ to pellet the DNA. The DNA was washed with $5 \mathrm{ml}$ of $70 \%$ ice cold ethanol by mix and centrifuged at $15000 \mathrm{rpm}$ for $5 \mathrm{~min}$. After the spin, the $70 \%$ ethanol was discarded and the pellet DNA was air dried for $15 \mathrm{~min}$. Then the DNA was dissolved in an appropriate amount of $\mathrm{dH}_{2} \mathrm{O}$. The yeast DNA was sonified at $20 \%$ output of the sonifier for $20 \mathrm{sec}, 3$ times. Its concentration was measured with spectrophotometer (2.1.1). Yeast DNA was saved at $-20^{\circ} \mathrm{C}$ in small aliquots for later use in the pre-clear step of oligo precipitation assay (2.2.4.1).

\subsubsection{Phenol-chloroform extraction of DNA}

To purify DNA-samples from hydrophobic impurities like lipids or proteins, the samples were treated with phenol and ethanol-precipitated:

The total volume of the DNA-sample was set to $100 \mu$ l with TE-buffer (pH 8.0), $200 \mu$ phenol was added and the sample was mixed (Vortex) and centrifuged at max speed for 5 min (Eppendorf microfuge). The upper aqueous phase was pipetted off into a new microcentrifuge tube and $100 \mu \mathrm{l}$ TE were added to the remaining lower phenol phase to extract additional DNA. The two forming phases were mixed and centrifuged again and the aqueous phase was removed as before and combined with the first $100 \mu \mathrm{l}$ of DNA-fraction. After adding $200 \mu \mathrm{l}$ chloroform to the sample, it was mixed and centrifuged as before and the upper phase was pipetted into a new centrifuge tube.

To precipitate the DNA, $400 \mu \mathrm{l}$ ice cold ethanol and $1 \mu \mathrm{l}$ glycogen [20 $\mathrm{mg} / \mathrm{ml}]$ was added to the sample which was then incubated for $30 \mathrm{~min}$ at $-80^{\circ} \mathrm{C}$. After incubation, the sample was centrifuged at max speed for $20 \mathrm{~min}$, and the ethanol was discarded. The DNA-glycogen pellet was washed with $1 \mathrm{ml}$ of $70 \%$ ethanol and the sample was centrifuged for another 20 min at maximum speed. The ethanol was carefully pipetted off and the DNA was air-dried at room temperature. After the DNA was dry, the pellet was re-suspended in $30 \mu \mathrm{lof} \mathrm{dH}_{2} \mathrm{O}$. 


\subsubsection{Insertion of point mutations in DNA}

Point mutations were inserted into DNA using the QuikChange ${ }^{\text {TM }}$ Site-Directed Mutagenesis Kit protocol from Stratagene:

Site specific complementary primers, which both bind to the sequence to be mutated and carrying the desired point mutations were used to amplify DNA from a template plasmid. The enzyme Dpnl was then used to digest the methylated non-mutant template DNA but not the unmethylated nicked circular strands that were amplified from it by the polymerase. The undigested DNA was then transformed into competent $\mathrm{XL}-1$ blue cells, which repaired the nicks, forming mutated plasmid-DNA to be analyzed by sequencing. The DNA of those clones which carried the specific point-mutated plasmid was then restriction-digested and re-cloned into a new plasmid to avoid complications due to unwanted mutations within the old vector during PCR. The procedure was done following the settings in the protocol of the manufacturer, except that ${ }^{E x}$ Taq-Polymerase (TaKaRa, $5 \mathrm{U} / \mu \mathrm{l}$ ) was used as polymerase and $5 \mu \mathrm{l}$ of the amplified sample was added to $100 \mu \mathrm{l}$ XL-1 blue bacteria suspension for transformation.

\subsubsection{Isolation of RNA out of S2 cells}

For isolation of RNA out of Schneider S2 cells, the RNA midiprep kit from Qiagen was used. The experiment was done following the manufacturers protocol and the total amount of cells used per preparation was within the recommended range of $3-4 \times 10^{7}$ cells.

\subsubsection{Isolation of genomic DNA out of S2 cells}

Genomic DNA of Schneider S2 cells was isolated with the Dneasy Blood \& Tissue Kit (2.1.2) of Qiagen. 1 to 2 million S2 cells was used for each preparation. The isolation was performed following the protocol of the manufacturer. The DNA has always been eluated from the column using $200 \mu \mathrm{l}$ eluation buffer of the manufacturer.

\subsubsection{Methylation sensitive enzyme analysis}

To analysis the DNA methylation of RBF gene in the S2 cells, $120 \mu$ genomic DNA from S2 cells (2.2.1.17) was first digested with $2.5 \mu \mathrm{l} \mathrm{HindIII} \mathrm{(NEB)} \mathrm{in} \mathrm{a} \mathrm{total} 150 \mu \mathrm{l}$ volume at $37^{\circ} \mathrm{C}$ for overnight. Digested DNA was purified with the PCR purification Kit (2.1.2) and eluted with $60 \mu \mathrm{l}$ elution buffer of the manufacturer. $10 \mu \mathrm{l}$ purified DNA was digested with 2-5 U Hpall (NEB restriction enzyme targeting to CCGG but inhibited by the methylation at the cytosine of the CG dinucleotide) in a total $20 \mu \mathrm{l}$ volume at $37^{\circ} \mathrm{C}$ for overnight. Un-digested DNA and DNA digested with Mspl (restriction enzyme targeting to CCGG and not sensitive to methylation) were taken as control. After 1 to 3 or 1 to 10 diluted, digested DNA and control DNA were detected by PCR with primers targeting to regions of interest. PCR product was applied to a $1.5 \%$ agraose gel to run for 45 min at 160v. The result was checked with the Eagle Eye II still video system. 


\subsubsection{Bisulfite sequencing}

Bisulfite sequencing was also applied in the detection of genomic DNA methylation. After digested with HindIII and purified, $20 \mu \mathrm{I}$ S2 DNA sample was applied to the EpiTect Bisulfite Kit (2.1.2) to be treated with bisulfite and subsequently cleaned up. The bisulfite treatment will convert the cytosine residues to uracil, but leaves 5-methyl cytosine residues unaffected. It thus introduces specific changes in the DNA sequence that depends on the methylation status of individual cytosine residues. Bisulfite treated DNA was applied to 2 rounds of PCR amplification to detect the DNA methylation. After 30 cycles of PCR amplification with first round primers (2.1.8.7), product from this PCR was used as template for the next 30 cycles of PCR with the primers for second amplification (2.1.8.7). Product from second PCR was purified with the PCR purification kit or Gel Cleanup Kit (2.1.2) to recover the DNA fragment of interest. The DNA was ligated with the pCR2.1TOPO vector of the TOPO TA cloning kit (2.1.2). XL-1 blue competent cells were transformed with the ligation product and transformed cells were spread onto an ampicillin LB agar plates to grow single colonies. Colonies were picked from the plates and transferred to $5 \mathrm{ml}$ ampicillin LB media. After overnight shaking at $37^{\circ} \mathrm{C}$, the culture was used for plasmid miniprep (2.2.1.12.2). Purified plasmids were sent for sequence with T7 sequencing primer. The sequence was compared to the original sequence of interest to identify the methylation by recognizing cytosines that are not converted to thymidines. For each sample detected for methylation with the BS sequencing, 10 to 13 colonies were sequenced and the methylation was scored as the percentage of the number of total methylated cytosine in all colonies to the presumptive total number of cytosines (which is the number of cytosines in the original sequence timed by the number of colonies).

\subsubsection{Labeling RNA with digoxigenin}

To prepare the digoxigenin labeled RNA probe for the in situ hybridization, the Roche DIG RNA labeling Kit was used. Template DNAs were pCR2.1TOPO-RBF-500, pCR2.1TOPO-MDU-500 and pCR2.1TOPO-dDNMT2-500 (2.1.9.2). The template DNAs were linearized by digestion with Spe1. The labeling was performed according to the protocol of the manufacturer and labeled RNA is stored at $-80^{\circ} \mathrm{C}$ for the following in situ hybridization. 


\subsubsection{Analysis and Manipulation of Proteins}

\subsubsection{SDS-polyacrylamide-gelelectrophoresis (SDS-PAGE)}

The separation of proteins according to their molecular weight was done via discontinual SDSpolyacrylamide-gel electrophoresis following the method of Laemmli (Laemmli 1970): Depending on the molecular weight of the separated proteins, matrixes of concentrations between $6-18 \%$ polymerised acrylamide were created:

Two glass plates of specific size were assembled, which were divided from each other by two vertical spacers of specific width $(0.75-1.5 \mathrm{~mm})$ on the sides and sealed on the bottom by another spacer (custom maxigel system) or a rubber mat (BioRad Protean minigel system). The resolving-gel (2.1.11.4) was then pipetted between the glass plates to polymerise at room temperature. Isopropanol was filled above the liquid resolving-gel solution before polymerisation to create a flat resolving-gel surface. After the gel was polymerised, the isopropanol was discarded, the gel surface was washed with MilliQ- $\mathrm{dH}_{2} \mathrm{O}$ and the lower concentrated stacking-gel (2.1.11.4) was poured on top of the resolving-gel. Before the stacking-gel could polymerise, a comb of sample-specific length and width was stuck into the stacking-gel, creating slots for loading the proteins into the gel. Protein samples were boiled in SDS-loading buffer at $100^{\circ} \mathrm{C}$ for 5 min before they were loaded on the gel to disrupt the spatial structure (secondary, tertiary and quaternary structures) of the proteins within the $\beta$-mercaptoethanol-containing buffer and to anneal the SDS to create an overall negative charge according to the size of the protein for the electrophoresis separation in the gel.

The electrophoresis was started at $80 \mathrm{~V}$ (mini-gels) to $100 \mathrm{~V}$ (maxi-gels) to stack the proteins within the stacking-gel and set to $150 \mathrm{~V}$ (mini-gels) to $200 \mathrm{~V}$ (maxi-gels) once the proteins entered the resolving-gel.

\subsubsection{Coomassie-staining of SDS-PAGE gels}

To visualize electrophoretically separated proteins in a SDS-gel, the gels were incubated for 30 min to overnight in Coomassie-staining solution (2.1.11.4). Afterwards the gels were incubated with several washes of destaining-solution (2.1.11.4) until bands became visible. The gel was washed with $\mathrm{dH}_{2} \mathrm{O}$, documented and dried onto Whatman-paper with a vacuum gel dryer (2.1.1) at $80^{\circ} \mathrm{C}$ for $1-2 \mathrm{~h}$.

\subsubsection{Silver staining of SDS-PAGE gels}

Silver staining allows a detection of proteins which is $10-100 \times$ more sensitive than Coomassie staining:

The gels were incubated in fixative 1 (for all solutions used, see 2.1.11.5) for $1 \mathrm{~h}$ and then again $1 \mathrm{~h}$ in fixative 2. After fixation of the protein bands, the gels were washed in Milli-Q $\mathrm{dH}_{2} \mathrm{O} 4 \mathrm{x}$ for $15 \mathrm{~min}$. The gel was sensitized for $1 \mathrm{~min}$ in $0.1 \mathrm{mM}$ sodium thiosulfate solution and rinsed $3 x$ for $20 \mathrm{sec}$ in Milli-Q $\mathrm{dH}_{2} \mathrm{O}$. To stain the gel, it was incubated in silvernitrate-solution for at least 30 min and afterwards washed again in Milli-Q $\mathrm{dH}_{2} \mathrm{O}$ for $1 \mathrm{~min}$. The gel was placed into developersolution for up to 30 min to visualize faint protein bands. When the gel was stained at the desired intensity, the reaction was stopped by placing the gel into stop-solution for $10 \mathrm{~min}$. The gels were 
documented afterwards and stored in $1 \%$ acetic acid (Milli-Q $\left.\mathrm{dH}_{2} \mathrm{O}\right)$ at $4{ }^{\circ} \mathrm{C}$ or dried onto Whatman-paper as in 2.2.2.2.

\subsubsection{Immunodetection of SDS-PAGE proteins (Western-blot)}

The power of the technique lies in the simultaneous detection of a specific protein by means of its antigenicity, and its molecular mass: proteins are first separated by mass in the SDS-PAGE, then specifically detected in the immunoassay step:

SDS-PAGE separated proteins can be transferred out of the gel onto a membrane in an electrical field without changing the separation pattern of the bands. Once on the membrane, protein bands can be immunologically assigned to specific proteins using specific antibodies in various immunodetection methods.

\subsection{Western-blot and primary antibody}

Transfer-membranes used for the Western-blot analysis were either nitrocellulose- or PVDFmembranes. PVDF-membranes needed to be activated by a short incubation in methanol before the blotting procedure and were then, like the nitrocellulose membranes, equilibrated in transfer buffer. Two different transfer buffers (blotting buffers) were used, transfer buffer II (Harlow and Lane 1999) for proteins above $150 \mathrm{kDa}$ and transferbuffer I for smaller sized proteins.

Proteins were blotted using BioRad's Mini Trans-Blot transfer cell, at $300 \mathrm{~mA}$ (around $5 \mathrm{~mA} / \mathrm{cm}^{2}$ gel-surface). The blotting setup ("blotting sandwich", in blotting direction left to right) consisted of one fiber pad, three Whatman-filterpapers, SDS-PAGE gel, membrane, another three Whatmanfilterpapers and the second fiber pad. If necessary, the blot was checked by staining the proteins on the membrane with Ponceau $S$ and marking their position by piercing the membrane with a sterile needle. The membrane was incubated in blocking-solution $(5 \%$ skim milk powder in TBST-buffer, 2.1.11.2) for $1 \mathrm{~h}$ at room temperature. The blocking-solution was discarded, the membrane was washed $3 x$ for $10 \mathrm{~min}$ in TBST and incubated in the primary-antibody-TBSTdilution (set according to the antibody manufacturers protocol) for $1 \mathrm{~h}$ at room temperature or at $4^{\circ} \mathrm{C}$ over night. To remove remaining primary antibody, the membrane was washed again in TBST as before. The membrane was now ready to be incubated with the secondary antibody.

\subsection{Immunodetection with alkaline phosphatase}

In this type of immunodetection, the secondary antibody was coupled to the enzyme alkaline phosphatase (AP) and was diluted in TBST according to the manufacturer's protocol. After incubation with the secondary-antibody-TBST-dilution at room temperature for $1 \mathrm{~h}$, the membrane was washed again $3 x$ for 10 min with TBST and then incubated in AP-buffer (2.1.11.2) with $4.5 \mu \mathrm{l} / \mathrm{ml} \mathrm{NBT}$ and $3.5 \mu \mathrm{l} / \mathrm{ml} \mathrm{BCIP}$ until the bands became visible and the reaction was stopped then by adding $\mathrm{dH}_{2} \mathrm{O}$.

\subsection{Immunodetection with chemiluminescence (ECL-Plus-Kit)}

The ECL-Plus-Kit (Amersham) uses biotinylated antibodies to detect proteins via chemiluminescence through the enzyme horse-raddish-peroxidase (HRP). After incubation with the secondary-antibody-TBST-dilution and washing in TBST 3x for $10 \mathrm{~min}$, the membrane was 
incubated in streptavidin-HRP solution (1:3000 in TBST) for $1 \mathrm{~h}$ at room temperature. The membrane was washed again in TBST as before and put in between two plastic foils with $1 \mathrm{ml}$ of the ECL-solution (according to the manufacturer's protocol). The fluorescence signals were detected using a Kodak X-Omat film in the darkroom within 5 min to $1 \mathrm{~h}$.

\subsubsection{Expression and affinity precipitation of recombinant Proteins out of E Coli}

\subsubsection{Expression of recombinant proteins in $E$ coli}

Expression of recombinant proteins in E coli is performed with the pGEX2TKN prokaryotic expression vector and its derivates (2.1.9.1. and 2.1.9.2) together with BL21(DE3)pLysS (2.1.6) $E$ Coli competent cells that enable efficient expression of heterologous proteins in the $E$ Coli. pGEX2TKN vector contains a GST gene coding region under the control of tac promoter which is chemically inducible by IPTG. Gene of interest is subcloned into the pGEX2TKN vector so that it's downstream and in code with the GST gene. After transforming the BL21(DE3)pLysS (2.1.6) $E$ Coli competent cells, the whole system, induced by IPTG, allows for the high level expression of the gene as a GST fusion protein.

To express the recombinant protein, the competent BL21(DE3)pLysS cells were transformed with recombinant pGEX2TKN (or void pGEX2TKN as a control) and single colonies were picked with a pipet tip, transferred into a bacteria-culture vial with $50 \mathrm{ml} \mathrm{LB}$-media containing $0.1 \mathrm{mg} / \mathrm{ml}$ ampicillin and were grown over night in an incubator-shaker. The next morning (12-16 $\mathrm{h}$ later), the overnight culture was transferred into $1000 \mathrm{ml}$ LB-media at the ratio of 1:25. The new culture was incubated in the $37^{\circ} \mathrm{C}$ shaker and the OD of the culture was monitored. IPTG was added to a final concentration of 0.1-1 mM when the OD value reached 0.4-0.6 to induce the expression. After that, the culture was kept incubated in the $37^{\circ} \mathrm{C}$ shaker for $3-5 \mathrm{~h}$ for the maximum production of the desired proteins. Then, the culture was centrifuged at $6000 \mathrm{rpm}$ for $15 \mathrm{~min}$ to spin down the bacteria. The supernatant was discarded and the bacteria were directly used for the following purification step or stored at $-80^{\circ} \mathrm{C}$.

\subsubsection{Preparation of the protein extract from $E$ Coli}

To separate the proteins from other cell components like DNA or lipids, protein extract is prepared with the following procedures (all steps were carried out at $4^{\circ} \mathrm{C}$ except otherwise indicated);

The bacteria from 2.2.3.1 were resuspended in the $0.5 \mathrm{M} \mathrm{NaCl} 1 \times \mathrm{NEGN}$ buffer (2.1.11.2) at the ratio of $5 \mathrm{ml}$ buffer to $100 \mathrm{ml}$ of bacteria culture. Then it was frozen in the liquid nitrogen and thawed twice. A grain of lysozyme (2.1.3) powder is added to the resuspended bacteria before it's stirred for $30 \mathrm{~min}$ in the cold room. Then the bacteria were broken with sonifier (2.1.1) at its $30 \%$ output and for $20 \mathrm{sec}$. The sonification was repeated for 3 times. Then the mixture was centrifuged at $15,000 \mathrm{rpm}$ for $15 \mathrm{~min}$. The pellet was discarded and the supernatant was subsequently passed through a $0.45 \mu \mathrm{m}$ and a $0.20 \mu \mathrm{m}$ filter before kept as the protein extract by fast freezing in the liquid nitrogen. 


\subsubsection{Affinity precipitation of the GST-proteins}

The high affinity of GST (glutathione S-transferase) to glutathione allows for the specific purification of the GST fusion proteins. To purify the recombinant protein from $1 \mathrm{ml}$ of extract, the slurry of glutathione sepharose beads were resuspended to a homogenized state, then $20 \mu l$ glutathione sepharose beads were pipetted to a $1.5 \mathrm{ml}$ ependorf tube and washed 2 times with $1 \mathrm{ml} 0.5 \mathrm{M} \mathrm{NaCl} 1 x$ NEGN buffer (2.1.11.2) by mix and spinning at $1000 \mathrm{rpm}$ for $2 \mathrm{~min}$, then the extract was applied to the beads and the mixture was incubated at $4^{\circ} \mathrm{C}$ for $4 \mathrm{~h}$. To remove nonspecific binding, the beads were washed $4 \mathrm{x}$ with $1 \mathrm{ml} 0.5 \mathrm{M} \mathrm{NaCl} 1 \mathrm{x}$ NEGN buffer in the same way like just mentioned. The beads containing the GST fusion protein were applied for the in vitro methyl DNA pull-down assay.

\subsubsection{DNA pull-down assay}

\subsubsection{Precipitation of Oligo DNA with GST fusion protein}

To demonstrate the interaction between methyl DNA and proteins of interest (e.g. the protein encoded by the MBD domain of MDU), a new method is developed in our lab. The principal idea of the method is to associate radiolabeled double strand DNA oligos (2.2.1.3 and 2.2.1.4) with immobilized protein in an appropriate buffer so that the interaction between DNA and protein will enable the "precipitation" of the oligo. Precipitated DNA is purified with the method described in 2.2.1.13. And the amount of the DNA is determined by autoradiography.

To carry out the assay, protein of interest was expressed in the $E$ Coli as a GST fusion protein and the protein extract was prepared (2.2.3.1 and 2.2.3.2). The extract was then applied to glutathione sepharose beads so that the GST fusion protein will bind to the affinity matrix and immobilized (2.2.3.3). In order to prevent non-specific DNA binding to the beads in the precipitation assay, $100 \mu \mathrm{l}$ of protein bound glutathione sepharose beads were incubated in 500 $\mu \mathrm{l}$ of $1 \mathrm{x}$ binding buffer (2.1.11.2) with $0.5 \mu \mathrm{g} / \mu \mathrm{l}$ yeast DNA at $4^{\circ} \mathrm{C}$ for $3 \mathrm{~h}$ by constant rotating. The beads were then washed $2 x$ with $1 x$ binding buffer (2.1.11.2) and the volume was adjusted to $100 \mu \mathrm{l}$ again with $1 \mathrm{x}$ binding buffer. $10 \mu \mathrm{l}$ of pre-incubated glutathione sepharose beads bound with GST fusion protein (or GST alone as a control) were transferred to a $1.5 \mathrm{ml}$ Eppendorf tube by pepitting with an end cut tip. $1 \mu$ l of the ${ }^{32} \mathrm{P}$ labeled double strand oligo DNAs (2.2.1.3 and 2.2.1.4) was applied to the beads together with $0.5 \mathrm{ml} 1 \mathrm{x}$ binding buffer (2.1.11.2). The mixture was incubated at $4^{\circ} \mathrm{C}$ for $4 \mathrm{~h}$ by constant rotating. After the incubation, the beads were washed $4 \mathrm{x}$ with $0.5 \mathrm{M} \mathrm{NaCl} 1 \mathrm{x}$ binding buffer (2.1.11.2) and $2 x$ with TE buffer (2.1.11.2). Then the precipitated DNA was recovered with the phenol/chloroform method (2.2.1.13).

\subsubsection{Detection of the precipitated oligo DNA}

To detect the radioactive oligo DNA, first the oligo DNA was applied to a nondenaturing polyacrylamide gel electrophoresis. To do that, a nondenaturing polyacrylamide gel was made in the way similar to the method in 2.2.2.1. Since no stacking gel is needed here, a comb was stuck into the nondenaturing polyacrylamide gel directly after it was cast to create slots for loading the oligo DNA samples into the gel. 1/10 volume of the EMSA gel-loading buffer (2.1.11.2) was mixed with the oligo DNA sample before it was loaded. The electrophoresis was carried out at $150 \mathrm{v}$ for 1.5 to $2 \mathrm{~h}$ until the Bromophenol blue of the gel-loading buffer is $1-2 \mathrm{~cm}$ from the end of 
the gel. Then the gel was transferred on to 3MM Whatman paper, covered with a plastic film and dried using a vacuum gel dryer (2.1.1) for $2 \mathrm{~h}$.

The dried gel was fixed with sticky bands in an intensification cassette. In the dark room, A X$\mathrm{OMAT}^{\mathrm{TM}}$ autoradiography film was put onto the gel and the cassette was closed to seal. The film was exposed for over night at $-80^{\circ} \mathrm{C}$, and then it was visualized and fixed with Autoradiography developer (2.1.1).

\subsubsection{Expression and immunoprecipitation of recombinant Proteins}

\section{out of Sf9-cells}

For expression of the tag-fusion proteins in Spodoptera frugiperda (Sf9) cells, the Baculo virussystem was used:

pVL-Baculo vectors were cloned (2.1.9.2) and the specific viruses (2.1.10) were created. For other proteins, certain Baculo viruses already existed (F. Sauer).

\subsubsection{Growing Sf9-cells in cell culture}

Frozen Sf9-cells $\left(-80^{\circ} \mathrm{C}\right)$ were quickly thawed at $37^{\circ} \mathrm{C}$ and transferred into a $100 \mathrm{ml}$ cell-culture flask containing $20 \mathrm{ml}$ serum-free Sf9-media (2.1.11.1). The cells were grown at $27^{\circ} \mathrm{C}$, changing the media every two days until a stable adherent layer of young cells had been formed. The cells were then transferred into a spinner-flask and kept as suspension culture, checking the amount of cells with a cell counting chamber (Neubauer) every two days. Cell numbers were kept at 1$2 \times 10^{6}$ cells per $\mathrm{ml}$ by repeated dilution of the culture with fresh media. To create backupsamples for growing new cell cultures, $1 \times 10^{6}$ cells per $\mathrm{ml}$ were slowly frozen to $-80^{\circ} \mathrm{C}$ in fetalbovine-serum containing $10 \%$ DMSO.

\subsubsection{Baculovirus-transfection}

\subsection{Primary transfection}

To create recombinant Baculo viruses in the primary transfection, $10^{6}$ Sf9-cells were pipetted in each cavity of a 6-well-plate and incubated at room temperature for 15-30 min to settle down and

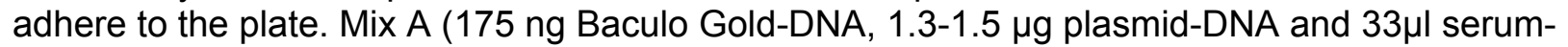
free Sf9-media) and Mix B (10 $\mu$ l Cellfectin reagent and $23 \mu \mathrm{l}$ serum-free Sf9-media) were prepared separately, then combined, mixed and incubated for $15 \mathrm{~min}$ at room temperature. After incubation, the Sf9-cells were washed $3 x$ with $1 \mathrm{ml}$ serum-free Sf9-media to remove old media and cell debris. $440 \mu \mathrm{l}$ serum-free Sf9-media was added to mixture AB and the complete transfection-solution was added to the cells. The plate was sealed with parafilm to prevent dehydration and incubated on a rocker at low speed for $15 \mathrm{~h}$ at room temperature. The transfection-solution was pipetted off and the transfected cells were washed once with serumfree Sf9-media. $2 \mathrm{ml}$ media was added to the cells which were sealed again and incubated at $27^{\circ} \mathrm{C}$ for 5 days. The cells were resuspended and the solution was centrifuged at $1000 \mathrm{rpm}$ for 5 min at $4^{\circ} \mathrm{C}$ (Eppendorf microfuge). The supernatant was taken off and stored at $-80^{\circ} \mathrm{C}$ or directly used for the amplification step. 


\subsection{Amplification and protein expression-test}

The virus-concentration after primary transfection is usually low and there is no detectable protein expression at this stage. Therefore, the virus needs to be amplified in order to determine if the protein is expressed or not.

$7.5 \times 10^{6} \mathrm{Sf9}$-cells were spread on a $10 \mathrm{~cm}$ cell culture dish and settled down for $15 \mathrm{~min}$. The old culture media was replaced with $2.5 \mathrm{ml}$ fresh Sf9-media and $1 \mathrm{ml}$ of the primary transfection supernatant was added. The plate was incubated on a rocker for $1 \mathrm{~h}$ at room temperature and then $10 \mathrm{ml}$ Sf9-media were added to the cells. The plate was incubated afterwards at $27^{\circ} \mathrm{C}$ for 5 days and the cells were resuspended and centrifuged as before in sterile $15 \mathrm{ml}$ tubes (Eppendorf centrifuge 5810R, rotor A-4-62). The supernatant was stored or used for a protein expression test.

To test if the transfection was successful and protein is expressed, $7.5 \times 10^{6}$ cells were spread on a $10 \mathrm{~cm}$ cell culture dish, settled down and washed as before. $0.5-1 \mathrm{ml}$ of the amplificationsupernatant was added and the solution was incubated for $1 \mathrm{~h}$, slowly rocking. $10 \mathrm{ml}$ media were added to the plate and the cells were incubated at $27^{\circ} \mathrm{C}$ for 2 days. After incubation, the media was removed, the cells were harvested and the protein purified for analysis with SDS-PAGE.

\subsection{Reamplification and protein expression}

After the transfected cells proved to express the protein, the virus was amplified again in order to be concentrated highly enough for a larger scale protein expression.

$12 \times 10^{6}$ Sf9-cells were spread on a $15 \mathrm{~cm}$ cell culture dish and settled down for $15 \mathrm{~min}$. The media was discarded and $5 \mathrm{ml}$ fresh Sf9-media and $50 \mu \mathrm{l}$ of the amplification supernatant was added. The plate was incubated on a rocker for $1 \mathrm{~h}$ at room temperature and $15 \mathrm{ml}$ Sf9-media has been added to the cells. The cells were incubated afterwards at $27^{\circ} \mathrm{C}$ for 5 days and then resuspended and centrifuged as before in sterile $50 \mathrm{ml}$ tubes. The supernatant was stored or used for protein expression.

To express the protein in a larger scale, amounts between $200-500 \mathrm{ml}$ of $10^{6} \mathrm{cells} / \mathrm{ml}$ were added into a $4 \mathrm{I}$ rlenmeyer flask. Between 1/50 - 1/100 volumes virus-re-amplificationsupernatant was added and the expression-culture incubated for $2-3$ days at $27^{\circ} \mathrm{C}$ in an incubator-shaker (2.1.1) before the cells were harvested and protein was purified.

\subsubsection{Immunoprecipitation of Sf9-expressed, FLAG-tagged proteins}

\subsection{Cell extracts of FLAG-protein-expressions}

The protein expressing cells were harvested by decanting (shaker cultures) or resuspending with a pipet (culture plates) into appropriate centrifuge tubes. They were centrifuged at $4^{\circ} \mathrm{C}$ and 2000 rpm (Eppendorf centrifuge 5810R, rotor A-4-62) for $10 \mathrm{~min}$, after this centrifugation step all other steps were done at $4^{\circ} \mathrm{C}$. The cell pellet was resuspended in $1 \mathrm{ml} /$ plate or $40 \mathrm{ml} /$ rlenmeyer $0.5 \mathrm{M}$ $\mathrm{NaCl}-\mathrm{HEMG}$-buffer (2.1.11.2) and twice frozen in liquid nitrogen and thawed again to break the cells. The sample was sonified $3 x$ for $20 \mathrm{sec}$ and the cell debris was removed by centrifugation for $15 \mathrm{~min}\left(4^{\circ} \mathrm{C}\right)$ at $15000 \mathrm{rpm}$ (Sorvall RC-5 superspeed centrifuge, SS34 rotor). The supernatant was subsequently passed through a $0.45 \mu \mathrm{m}$ and a $0.20 \mu \mathrm{m}$ filter. The filtrate was used directly for binding to FLAG-beads or was frozen in liquid nitrogen and stored in a low temperature freezer at $-80^{\circ} \mathrm{C}$. 


\subsection{Immunoprecipitation with FLAG-beads}

Anti-FLAG M2 affinity beads (FLAG-beads) were washed once with $0.5 \mathrm{M} \mathrm{NaCl}$-HEMG-buffer and incubated with the cell extract filtrate (2.2.5.3.1.) over night on a nutator at $4^{\circ} \mathrm{C}$. The sample was centrifuged for $2 \mathrm{~min}$ at $1000 \mathrm{rpm}\left(4^{\circ} \mathrm{C}\right)$ to pellet the beads. The harvested FLAG-beads were washed $4 \mathrm{x}$ with $0.5 \mathrm{M} \mathrm{NaCl}-\mathrm{HEMG}$-buffer and $2 \mathrm{x}$ with TE buffer by carefully centrifuging the beads as before. Then the beads can be stored at $4^{\circ} \mathrm{C}$. To elute the FLAG-tagged proteins off the beads, FLAG-peptide-solution [10mg/ml] was added in a relation of $1: 1$ to the beadsvolume and incubated at $4{ }^{\circ} \mathrm{C}$ on a nutator for at least $4 \mathrm{~h}$. Then the sample was centrifuged as before and the eluate was pipetted into a new sterile tube and frozen in liquid nitrogen. Or, the beads bound with FLAG fusion proteins can be directly applied to the downstream assays like histone methyltransferase assay.

\subsection{6 histone methyltransferase (HMT) assay and the detection by fluorography}

\subsubsection{Histone methyltransferase assay in solution}

The histone methyltransfearses can catalyze the transfer of one to three methyl groups from the cofactor S-Adenosyl methionine to lysine and arginine residues of histone proteins. To test the methyltransferase activity of certain presumptive epigenetic regulators like MDU, the in vitro histone methyltransferase assay was performed. $20 \mu \mathrm{l}$ of FLAG-beads bound with approximately $0.1-0.5 \mu \mathrm{g}$ of FLAG tagged proteins (2.2.5.3.2) were washed $2 \mathrm{x}$ with methyltransferase buffer (2.1.11.2). Then the reaction was set up by incubating the beads in methyltransferase buffer in the presence of $2 \mu \mathrm{Ci} \mathrm{S-adenosyl-I[methyl-3H]-methionine} \mathrm{(50-62} \mathrm{mCi} / \mathrm{mmol})$ and $1-5 \mu \mathrm{g}$ recombinant histones for $1 \mathrm{~h}$ at $30^{\circ} \mathrm{C}$. The reaction was stopped by adding $1 / 4$ of $4 \mathrm{x}$ SDSloading buffer and boiled at $95^{\circ} \mathrm{C}$ for $5 \mathrm{~min}$.

\subsubsection{Detection and analysis by fluorography}

The histone methylation catalysed by the presumptive HMT was detected with fluorography. In fluorography, radioactively labeled substances emit radiation that excites a molecule known as a scintillator. When the excited molecule relaxes to its ground state, it emits a photon of visible or ultraviolet light that is detected by photographic film. The reaction of 2.2.6.1 was loaded to and resolved by $15 \%$ SDS-PAGE (2.2.2.1) and analyzed by Coomassie. Stained gels were then incubated with the NAMP100V Amplify scintillator (2.1.3) for $30 \mathrm{~min}$ and dried for $1 \mathrm{~h}$ over filter paper with Gel Dryer (2.1.1). Radioactive signal from ${ }^{3} \mathrm{H}$ in dried gels were detected with autoradiography like described in 2.2.4.2. 


\subsubsection{Luciferase assay after $\mathrm{S} 2$ cell transfection}

\subsubsection{Growing Schneider S2 cells in cell culture}

Frozen S2 cells $\left(-80^{\circ} \mathrm{C}\right)$ were quickly thawed at $37^{\circ} \mathrm{C}$ and transferred into a $100 \mathrm{ml}$ cell-culture flask containing $20 \mathrm{ml}$ serum-free Sf9-media (2.1.11.1). The cells were grown at $27^{\circ} \mathrm{C}$, changing the media every two days until a stable adherent layer of young cells had been formed. The density of cells was checked with a cell counting chamber (Neubauer) every two days. Cell numbers were kept at $2-4 \times 10^{6}$ cells per $\mathrm{ml}$ by repeated dilution of the culture with fresh media. To create backup-samples for growing new cell cultures, $1 \times 10^{6}$ cells per $\mathrm{ml}$ were slowly frozen to $-80^{\circ} \mathrm{C}$ in fetal-bovine-serum containing $10 \%$ DMSO. The recombinant S2-tet-tk-luc cells were cultured in similar way while neomycin was added to the serum-free Sf9-media at a final concentration of $1 \mathrm{mg} / \mathrm{ml}$.

\subsubsection{Transfection of the $\mathrm{S} 2$ cells}

The S2 or S2- tet-tk-luc cells were transfected with cellfectin reagent (2.1.3), which has been specifically designed for transfecting insect cells. Mix A (1-4 $\mu$ g plasmid-DNA diluted with serum and antibiotics-free Sf9-media in a total $100 \mu \mathrm{l}$ volume) and Mix B $(2-10 \mu \mathrm{l}$ Cellfectin reagent diluted with serum and antibiotics-free Sf9-media in a total $100 \mu$ l volume) were separately set up in 2 Eppendorf tubes. Then the 2 were combined, mixed and incubated for 30 min at room temperature. At the mean time, S2 cells were counted and $3-6 \times 10^{6} \mathrm{~S} 2$ cells were washed once with serum and antibiotics-free Sf9-media. Then the cells were resuspended with $0.8 \mathrm{ml}$ serum and antibiotics-free Sf9-media. S2 cells were then mixed with A+B and were pipetted in each cavity of a 6-well-plate and incubated at room temperature for $6 \mathrm{~h}$. After incubation, $2 \mathrm{ml}$ serum and antibiotics-free Sf9-media was added to the cells. The plate was sealed with parafilm to prevent dehydration and incubated at $27^{\circ} \mathrm{C}$ for 2 days.

\subsubsection{Sorting the cells with flow cytometry}

After co-transfection of the S2 cells with a gene of interest and a marker plasmid (here the pAcGFP which expresses GFP protein), the cells were sent to the Core Instrumentation Facility (CIF) of the University of California, Riverside to be sorted with the FACSAria cell sorter (2.1.1) according to the fluorescent characteristics of each cell. 2 days after transfection, cells were resuspended with a cell scraper and the culture was washed once with serum and antibioticsfree Sf9-media. Then the cells were sorted with the cell sorter. The window of sorting was set so as over $90 \%$ of recovered cells were supposed to be fluorescent positive. 1-2 million cells were collected. To apply the cells in the following luciferase assay, sorted cells were spinned down at $100 \mathrm{rpm}$ for $5 \mathrm{~min}$ at $4^{\circ} \mathrm{C}$. The cells were resuspended in $1 \mathrm{ml} 1 \mathrm{x}$ PBS, and $200 \mu \mathrm{l}$ was used to check the cell density with a cell counting chamber (Neubauer). The rest of the cells were span down and used in the luciferase assay. 


\subsubsection{Luciferase assay}

Luciferase assays were performed to determine the luciferase expression in S2-tet-tk-luc cells (2.1.7). The principle of the assay is that luciferase catalyzes a light-producing reaction in the presence of the substrate luciferin. And the light intensity is proportional to luciferase concentration in a wide range (from $10^{-16} \mathrm{M}(10 \mathrm{pg} / \mathrm{L})$ to $10^{-8} \mathrm{M}(1 \mathrm{mg} / \mathrm{L})$ ). Thus the detection of light intensity represents the concentration of the luciferase. To carry out the luciferase assay, Sorted cells (2.2.7.3) were resuspended with $400 \mu$ of $1 x$ PBS together with $100 \mu \mathrm{l} 5 \mathrm{x}$ reporter lysis buffer from the Luciferase Assay System (2.1.2), frozen in liquid nitrogen and thawed. Then the lysate was span at $13000 \mathrm{rpm}$ for $5 \mathrm{~min}$ and the supernatant was transferred to a clean tube. $100 \mu \mathrm{l}$ of the supernatant was applied for light-detection with the luminometer (2.1.2). For each sample, the light-detection was repeated for 4 times and an average was taken. The final result was obtained by normalizing to the cell density of each sample.

\subsubsection{Antibody preparation}

\subsubsection{Development of antibodies}

To develop the anti-MDU (CG30426) antibody, 2 polypeptides were synthesised at Biosynthesis, namely $\mathrm{K} 796-1$ and $\mathrm{K} 796-2$. The amino acid sequence of $\mathrm{K} 796-1$ is $\mathrm{NH}_{2}$-YDDGYTQYVPHRDC$\mathrm{OH}$, which covers the 159-171 amino acid sequence of the presumptive MDU protein. The amino acid sequence of $\mathrm{K} 796-2$ is $\mathrm{NH}_{2}$-NNSTIYVDDENRC-OH, which covers the 351-362 amino acid sequence of the presumptive MDU protein. Both polypeptides were sent for developing monoclonal anti-MDU antibodies with the hybridoma method by Elisabeth Kremmer (GSF-Forschungszentrum, Institut für Molekulare Immunologie). The antibodies were tested for their specificity to the in vitro expressed FLAG-tagged MDU or $\triangle N$ MDU by Western-blot

(2.2.2.4). One antibody (named a-Full MDU) developed with the polypeptide K796-1 and the other one (named a- $\Delta \mathrm{N}$ MDU) developed with the polypeptide $\mathrm{K} 796-2$ were saved at $4^{\circ} \mathrm{C}$ for the following purification and XChIP assays.

To develop the anti-dDNMT2 (CG10692) antibody, a polypeptide named 7A53-2 was synthesised at Biosynthesis. The amino acid sequence of 7A53-2 is $\mathrm{NH}_{2-}$ GCQPHTRQGLQRDTEDKRSDAL-OH, which covers the 64-84 amino acid sequence of the presumptive dDNMT2 protein. The polypeptide was sent to Biosynthesis to develop polyclonal antibodies to dDNMT2 by immunizing 2 rabbits. Anti-sera from the 2 rabbits was collected at 6 and 8 weeks and was tested for its specificity to the in vitro expressed FLAG-tagged dDNMT2 by Western-blot (2.2.2.4). The rabbits were killed and the sera saved at week 10. Anti-sera from one rabbit (which has better specificity to dDNMT2 than the other one) was kept.

\subsubsection{Purification of the antibody with protein A or protein $G$ beads}

The monoclonal antibody from rat was purified with protein $\mathrm{G}$ agarose beads and the polyclonal antibody from rabbit was purified with protein A agarose beads. $200 \mu \mathrm{l}$ beads were washed in a $15 \mathrm{ml}$ tube with 1x PBS for 2 times by mixing and spinning at $1000 \mathrm{rpm}$ for $2 \mathrm{~min}$. Then $13 \mathrm{ml}$ crude antibody was added to the beads with $1.3 \mathrm{ml} 1 \mathrm{M}$ Tris $(\mathrm{pH} 8.0)$. The mixture was incubated at $4^{\circ} \mathrm{C}$ for overnight by constant rotating. The next morning the beads were span down and transferred to a Chromatography column. The beads were washed $4 x$ with $800 \mu l 100 \mathrm{mM}$ Tris 
(pH 8.0) and 4x with $800 \mu \mathrm{l} 10 \mathrm{mM}$ Tris (pH 8.0). To elute the antibody, $100 \mu \mathrm{l}$ of $100 \mathrm{mM}$ Glycine ( $\mathrm{pH} 3.0)$ was added slowly and gently to the column. The column was drained by gravity, and the eluate was collected in a $1.5 \mathrm{ml}$ microtube with $10 \mu \mathrm{l}$ pre-added $1 \mathrm{M}$ Tris $(\mathrm{pH} 8.0)$. The tube was gently flicked to mix the antibody with the $1 \mathrm{M}$ Tris. The elution step was repeated for 6 times. Recovered antibody was checked by SDS-PAGE. Eluates containing concentrated antibody were pooled together. $\mathrm{NaN}_{3}$ was added to the antibody to a final concentration of $0.02 \%$ and the antibody was stored at $4^{\circ} \mathrm{C}$.

\subsubsection{Immunoprecipitation assay}

\subsubsection{Cell lysate preparation}

To prepare lysate from adherent cells, cells were washed with ice-cold PBS. After drainning the PBS, ice-cold IP-lysis buffer was added to the cells $\left(1 \mathrm{ml}\right.$ per $10^{7}$ cells). Then the adherent cells were scraped off the dish using a cold plastic cell scraper and gently transferred into a precooled microcentrifuge tube (For cells cultured in suspension, appropriate amount of cell culture were harvested by centrifugation at $1000 \mathrm{rpm}$ for $5 \mathrm{~min}$ at $4^{\circ} \mathrm{C}$. The supernant was discarded and the cells were washed with $1 \times$ PBS $\left(1 \mathrm{ml}\right.$ per $10^{7}$ cells) by resuspension and centrifugation. After spin down the cells again, ice-cold IP-lysis buffer was added to the cells ( $1 \mathrm{ml}$ per $10^{7}$ cells) and the cells were resuspended by pepetting). Cells in IP-lysis buffer were frozen in liquid nitrogen and thawed twice. After sonification (20 sec per time and 3 times), cell suspension were spinned at $15000 \mathrm{rpm}$ for $15 \mathrm{~min}$ at $4^{\circ} \mathrm{C}$. The supernatant was transferred to a clean microcentrifuge tube and used for immunoprecipitation or stored at $-80^{\circ} \mathrm{C}$ after being frozen in liquid nitrogen.

\subsubsection{Immunoprecipitation}

To bind protein to its antibody, $1 \mathrm{mg}$ cell lysate ( or Drosophila embryo nuclear extract) were mixed with $1-5 \mu \mathrm{g}$ of antibody. The mixture was incubated on a nutator for $4 \mathrm{~h}$ at $4^{\circ} \mathrm{C}$. Then appropriate amount $(20-30 \mu \mathrm{l})$ protein A or protein $\mathrm{G}$ beads were added to the mixture to affinity precipitate the antibodies (Monoclonal antibody was affinity precipitated with protein G-coupled beads while polyclonal antibody was affinity precipitated with protein A-coupled beads). The lysate-beads mixture was incubated at $4^{\circ} \mathrm{C}$ under rotary agitation for 4 hours. After incubation, the protein $A$ or protein $G$ beads were spinned down (1000 rpm $2 \mathrm{~min}$ ). Then the supernatant was removed and saved at $-80^{\circ} \mathrm{C}$ after being frozen in liquid nitrogen. The beads were washed in IP-lysis buffer three times. Then they were washed two more times with TE buffer and the last supernatant was removed. 15-25 of 4x SDS-PAGE loading buffer and appropriate amount of water was added. The sample was mixed and boiled at $95-100^{\circ} \mathrm{C}$ for 5 minutes to denature the protein and separate it from the protein-A/G beads. Then it was spinned at $13000 \mathrm{rpm}$ for $5 \mathrm{~min}$. The supernatant containing the proteins was saved by freezing the samples at $-20^{\circ} \mathrm{C}$ or, it can be applied to a SDS-PAGE directly. 


\subsubsection{Crosslinked Chromatin Immunoprecipitation (XChIP)}

\subsubsection{Crosslinked cell extract preparation}

To prepare the Schneider S2 cell extract for XChIP, S2 cells were resuspended with a cell scraper and pooled in a $50 \mathrm{ml}$ tube (2.1.2) at the density of $3-6 \mathrm{x} 10^{6} / \mathrm{ml}$. 37\% Formaldehyde (crosslinker) was added to a final concentration of $1.8 \%$ and the cell suspension was vigorously shaken for $15 \mathrm{~min}$ on a shaker at room temperature. After the crosslinking, 1M Glycine was added to the cells to a final concentration of $125 \mathrm{mM}$ and incubated $5 \mathrm{~min}$ at room temperature. The cells were centrifuged at $1000 \mathrm{rpm}$ (Eppendorf centrifuge) for $5 \mathrm{~min}$ and the pellet was washed in pre-cooled 1x PBS. The sample was then centrifuged again at 1000rpm for 5 min and the pellet was resuspended in IP-lysis buffer of 2.1 .11 .2 ( $3 \mathrm{ml}$ per $200 \mathrm{ml}$ of cell pellet). The suspension was frozen and thawed twice in liquid nitrogen, sonified (6x for $20 \mathrm{sec}$ ) to an average fragment length of 700 base pairs. Afterwards the cell suspension was centrifuged at $15000 \mathrm{rpm}$ for $15 \mathrm{~min}$ and the supernatant (which contains cross-linked-chromatin) was transferred to a clean tube. This supernatant was used in the chromatin immuno-precipitation assay. To save the supernatant, it's frozen in liquid nitrogen and store in $-80^{\circ} \mathrm{C}$.

To prepare the Drosophila embryo cell extract for XChIP, appropriately staged embryos (0-12 h) were collected, washed with distil water and dechorionated with 3\% Sodium hypochloride for 90 seconds and rinsed with Wash buffer extensively. Then the embryos were weighed, and transferred to $50 \mathrm{ml}$ Falcon tubes at the ratio of $3 \mathrm{~g}$ embryo per tube. $10 \mathrm{ml}$ Binding buffer and 30 $\mathrm{ml}$ hepatane were added to the embryos. $37 \%$ formaldehyde was added to the mixture to a final concentration of $1.8 \%$. The embryo suspension was vigorously shaken for $15 \mathrm{~min}$ on a shaker at room temperature. After the crosslinking, the embryos were collected and transferred to with $1 \mathrm{x}$ PBS with $0.125 \mathrm{M}$ glycine. The embryo suspension was shaken for $5 \mathrm{~min}$ on a shaker at room temperature. Then the embryos were collected and washed with 1x PBS extensively. Embryos were transferred to IP-lysis buffer at the ratio of 1 volume embryo to 9 volume of IP-lysis buffer. 1 volume of glass beads (diameter $0.5 \mathrm{~mm}$ ) was added to the embryo-IP-lysis buffer mixture. The mixture was sonified ( $6 x$ for $20 \mathrm{sec}$ ) to shear the DNA to an average fragment length of 700 base pairs. Afterwards the suspension was centrifuged at $15000 \mathrm{rpm}$ for $15 \mathrm{~min}$ and the supernatant (which contains cross-linked-chromatin) was transferred to a clean tube. This supernatant was used in the chromatin immuno-precipitation assay. To save the supernatant, it's frozen in liquid nitrogen and store at $-80^{\circ} \mathrm{C}$.

\subsubsection{Immunoprecipitation of Crosslinked chromatin}

Protein A or Protein G beads (2.1.4) were used according to the nature of the antibody applied in the chromatin precipitation. All procedures were carried out at $4^{\circ} \mathrm{C}$ except otherwise indicated. Affinity beads were blocked with IP-lysis buffer (2.1.1.1), with BSA (1mg/ml, Roth) and salmon testis DNA $(1 \mathrm{mg} / \mathrm{ml})$ for $2-3 \mathrm{~h}$ and then washed $3 \mathrm{x}$ with XChIP-lysis buffer. $25 \mu \mathrm{l}$ blocked beads were added to each $1 \mathrm{ml}$ of the supernatant (2.2.10.1). The mixture was incubated for $4 \mathrm{~h}$ and then centrifuged at $1000 \mathrm{rpm}$ for 2 min to pellet the beads. Then the pre-cleaned supernatant was transferred to another $1.5 \mathrm{ml}$ microtube. $1-5 \mu \mathrm{g}$ specific antibodies (except control sample) were added to the pre-cleaned chromatin and the 2 were incubated over night. 10 to $20 \mu$ l of blocked beads were added to the samples from last step and the mixture was incubated for $8 \mathrm{~h}$. The samples were centrifuged at $1000 \mathrm{rpm}$ for $2 \mathrm{~min}$ to pellet the beads. Then the beads were washed 4x in IP-lysis buffer, 4x in IP1, 4x in IP2 and 4x in TE (pH 8.0). Washed 
beads were resuspended in 100 TE. RNaseA was added (final conc. $50 \mu \mathrm{g} / \mathrm{ml}$ ) and incubated at $37^{\circ} \mathrm{C}$ for $30 \mathrm{~min}$. $20 \%$ SDS was added to a final concentration of $0.5 \%$ and $50 \mathrm{mg} / \mathrm{ml}$ Proteinase$\mathrm{K}$ was added to a concentration of $0.5 \mathrm{mg} / \mathrm{ml}$. The samples were then incubated over night at $37^{\circ} \mathrm{C}$.

The samples were transferred to $65^{\circ} \mathrm{C}$ water bath and incubated there for $6 \mathrm{~h}$ to reverse the cross-links. To recover the DNA from precipitated chromatin, the samples were cleaned up with phenol-chloroform-extraction (2.2.1.14). The DNA was analysed with PCR.

\subsubsection{Manipulation of Drosophila melanogaster}

\subsubsection{Stockkeeping, matings and embryo collecting}

Drosophila stocks were cultured by periodic mass transfer of adults to fresh food. Bottles or vials are tapped on the pounding pad to shake flies away from the plug. The plug was rapidly removed, and the old culture was inverted over a fresh bottle or vial. Flies were tapped into the new vessel, and the two were rapidly separated and replugged. The frequency with which new subculture need to be established depends on the health and fecundity of the genotype, the temperature and the density of the cultures. Generally, they were kept at room temperature and were transferred to fresh food every 20 to 30 days.

To cross two fly strains, adult flies in the bottles of two strains were discarded. Virgin females from one strain were collected about $8 \mathrm{~h}$ after the adults were cleared and were crossed to males from the other strain by putting the females and males in the same vial at the ratio of 10 to 1 (female to male). The progenies were checked for phenotype.

To collect the embryo of desired fly strain, adult flies were transferred to the fly cages bottomed by apple- juice agar plates with yeast. Every $12 \mathrm{~h}$ the plates were replaced with fresh ones rapidly by turning the cage bottom up and tapping the plates to shake flies away from it. The embryos were removed from the plate by rinsing with $\mathrm{dH}_{2} \mathrm{O}$ and brushing with a drawing brush. The embryos were immediately applied for the following experiments or store at $-80^{\circ} \mathrm{C}$.

\subsubsection{Preparation of Drosophila embryo nuclear extract}

Appropriately staged embryos $(0-12 \mathrm{~h})$ were incubated at $25^{\circ} \mathrm{C}$ and collected from apple-juice agar plates. After this, all following steps were carried out on ice or in the cold room. Embryos were washed, dechorionated with 3\% Sodium hypochloride for 90 seconds and rinsed with Wash buffer extensively. Then distil water was used to remove excess Wash buffer and bleach. Embryos were dried and weighed. Nuclear Buffer 1 was combined with the embryos at the ratio of $3 \mathrm{ml}$ Nuclear Buffer 1 to $1 \mathrm{~g}$ embryos. The embryos were disrupted with a single passage through the Yamato LH-22 homogenizer at $1000 \mathrm{rpm}$ (setting=100). The homogenate was filtered with Miracloth and the flow-through was collected with GSA bottles. The remaining debris retained by the Miracloth was washed with additional Nuclear Buffer 1 ( $2 \mathrm{ml} / \mathrm{g}$ of embryos). The final volume of Nuclear Buffer 1 should be about $5 \mathrm{ml} / \mathrm{g}$ embryos. To pellet the nuclei, the GSA bottles were span at $8000 \mathrm{rpm}$ for $15 \mathrm{~min}$. Supernatant was carefully decanted and the lipids were wiped off the walls of the centrifuge bottles with Kimwipes. The nuclei were suspended in 5 $\mathrm{ml} / \mathrm{g}$ embryos of Buffer 1. A $40 \mathrm{ml}$ Dounce with a B pestle was used to disperse the nuclei. Nuclei were transferred to clean GSA bottles and pelleted again in the GSA rotor at 8000 rpm for $15 \mathrm{~min}$. Supernatant was discarded and the nuclei were resuspended with $0.2 \mathrm{ml} / \mathrm{g}$ embryos of 
IP-Lysis buffer. After 2 times of freezing and thawing, the suspension was sonified 3 times at $40 \%$ output for $20 \mathrm{sec}$. Then it was span at $15000 \mathrm{rpm}$ for $15 \mathrm{~min}$. The supernatant was filtered with $0.45 \mu \mathrm{m}$ and $0.2 \mu \mathrm{m}$ filter sequentially and stored at $-80^{\circ} \mathrm{C}$.

\subsubsection{In situ Hybridizations}

Appropriately staged embryos (0-4, 9-12 and $21-24 \mathrm{~h}$ ) were incubated at $25^{\circ} \mathrm{C}$ and collected from apple-juice agar plates. They were washed, dechorionated with $3 \%$ Sodium hypochloride and washed extensively with $0.1 \%$ TritonX100 and $\mathrm{dH}_{2} \mathrm{O}$. Embryos were fixated with Fix-solution and Heptan for 20 min on a rocking platform, devitellinaized by 1 min vortexing with methanol, and washed extensively with methanol.

Embryos were again fixated with 1:1 Fix-solution/PBT for $20 \mathrm{~min}$, then rinsed thrice and washed by rotating for 5 min twice with PBT. Then, a limited Proteinase $\mathrm{K}$ digest $(5 \mathrm{~min}$ with $5 \mathrm{mg} / \mathrm{ml}$ Proteinase $K$ at 1:500 final dilution with PBT: $5 \mu \mathrm{l}$ of stock aliquots in $1 \mathrm{ml}$ PBT and $0.5 \mathrm{ml}$ embryos) was performed to make the tissues more accessible to the probes. The embryos were again washed, fixated and washed as previously described. Hybridization conditions were gradually taken to the optimum by rinsing sequentially with $1: 1 \mathrm{HybeB} / \mathrm{PET}$, HybeB and Hybe solutions. From this step to the PBT washes, embryos were handled in a metal block heated at $65^{\circ} \mathrm{C}$. Embryos were pre-hybridised with Hybe-solution at $65^{\circ} \mathrm{C}$ for $30-60 \mathrm{~min}$. Then, $5 \mu \mathrm{l}$ of DIGlabeled probe were assed in $30 \mu \mathrm{l} \mathrm{Hybe-solution} \mathrm{preheated} \mathrm{to} 65^{\circ} \mathrm{C}$ and the preparations were hybridised overnight at $65^{\circ} \mathrm{C}$. After washing thrice for 15 min with $\mathrm{HybeB}$ at $65^{\circ} \mathrm{C}$ and twice rinsing and thrice washing $(5,10$ and $20 \mathrm{~min}$ ) with PBT, the embryos were incubated with preabsorbed a-DIG-AKP Fab-fragments for $90 \mathrm{~min}$ at room temperature and washed extensively with PBT. To develop, embryos were washed thrice 5 min with AP buffer and incubated for 15 min to $2 \mathrm{~h}$ with supplemented AP buffer. Reactions were stopped by extensive PBT washing, twice washing with $70 \%$ ethanol, twice with $100 \%$ ethanol and conserved at $-20^{\circ} \mathrm{C}$. Stained embryos were then mixed with $200 \mathrm{ml}$ mount solution and mounted over microscope slides. 


\section{Results}

This study aimed at the dissection of the role of the MBD/SET protein MDU in gene silencing in Drosophila.

DNA methylation is present in various organisms such as plants, vertebrates and insects. DNA methylation and histone methylation are two key players in epigenetic gene expression regulation. MBD domain proteins, Histone methyltransferases, DNA methyltransferases, histone acetyltransferases and histone deacetylases are involved in gene silencing (Dobosy and Selker 2001; Bannister and Kouzarides 2004; Herceg and Wang 2005).

In Drosophila, DNA methylation appears at early embryo stages and disappears when the fly reaches adulthood (Lyko et al., 2000a; Gowher et al., 2000). Because DNA methylation in Drosophila has only been discovered in recent years and the level of DNA methylation in Drosophila is much lower than that in vertebrates or plants (Lyko et al., 2000a, Gowher et al., 2000), it is not clear how de novo DNA methylation is initiated in Drosophila, what gene(s) is under the control of DNA methylation and how DNA methylation is integrated into the epigenetic regulation network to bring about transcriptional repression of target genes.

MDU (CG30426) contains a MBD (aa 401-471) domain and a SET domain (aa 601-823) -- the landmark motif of lysine-specific HMTs. MDU methylates H3K9 in vitro and in vivo (MoraBermúdez 2002; Seum et al., 2007; Tzeng et al., 2007). The function of the MBD remains unknown. The presence of SET and MBD domains in MDU strongly suggests a role for MDU in regulation of gene expression and DNA methylation in Drosophila. To test this hypothesis, I performed functional in vitro and in vivo assays.

DNA pull-down assays assessed the interaction of the MBD of MDU with methylated DNA. The results of these assays indicate that the MBD of MDU binds methylated CpA motifs

Western blot and in vitro HMT-assays were used to define the target specificity for the SET domain of MDU. The results reveal that MDU tri-methylates H3K9.

I used transient transfection assays to dissect the transcriptional regulatory potential of MDU in Drosophila tissue culture cells. The results suggest that MDU-mediated tri-methylation of H3K9 mediates silencing of reporter gene expression in Drosophila cells.

Tissue culture, in combination with RT-PCR and chromatin immunoprecipitation assays, was used to identify target genes for MDU. One of the identified MDU target genes is Rbf. MDU represses $R b f$ transcription in Drosophila tissue-cultured cells. 
The results of RNA interference (RNAi) assays with MDU expression destroyed in eye imaginal discs of Drosophila third-instar larvae indicate that MDU represses Rbf expression in eye imaginal discs.

A recent study has demonstrated that repression of $R b f$ expression involves DNA methylation (Ferres-Marco et al., 2006). To assess the role of MDU in silencing and DNA methylation of $R b f$, transient transfection assays with wild type and mutant versions of MDU were used to assess the role of MDU in Rbf silencing. The obtained results support a model in which MDU-mediated tri-methylation of H3K9 initiates DNA methylation by dDNMT2 at the Rbf locus, culminating in silencing of $R b f$ expression.

The results of this study uncover the mechanisms for de novo DNA methylation in Drosophila and a role for members of the SET/MBD domain family of epigenetic regulators in control of cell proliferation in development and diseases.

In summary, this study focused on the molecular mechanism of MDU-mediated transcription repression by dissecting the function of its MBD and SET domains and their roles in the silencing of $R b f$.

\subsection{Generation of antibodies}

\subsubsection{Anti-MDU antibody}

To investigate the expression of MDU in Drosophila embryos by immunoprecipitation and to assess the role of MDU in transcription repression with XChIP, anti-MDU antibody was developed as described (2.2.8.1).

This antibody was developed with use of the antigen peptide $\mathrm{NH}_{2}$-NNSTIYVDDENRC$\mathrm{OH}$ (aa 351-362) of the presumptive MDU protein. The antibody was tested for the ability to precipitate recombinant FLAG- $\triangle$ NMDU from the SF9 cell extract. Extract from Sf9 cells expressing FLAG- $\triangle$ NMDU or cell extract from Sf9 cells was immunoprecipitated with anti-MDU antibody or rat serum. Precipitated proteins were detected by Western Blot using anti-FLAG antibody. The anti-MDU antibody specifically precipitated recombinant MDU (Figure 8). 


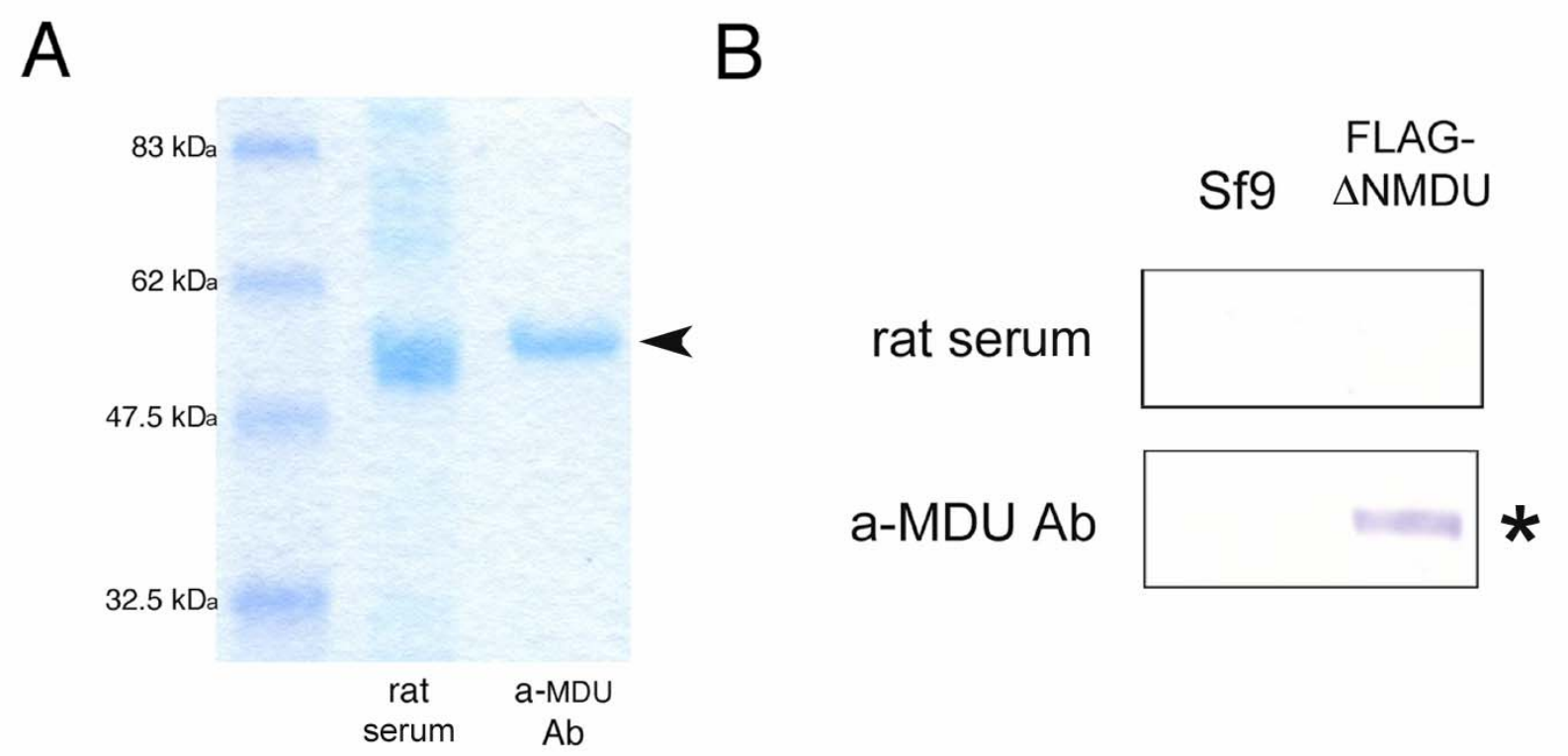

Figure 8. Functional characterization of monoclonal antibody recognizing MDU. (A) Coomassie blue-stained polyacrylamide gel showing antibodies immunoprecipitated from rat serum and hybridoma culture supernatant. Hybridoma cells producing antibody to MDU were cultivated for 3-5 weeks. Cells were removed and the culture supernatant was analyzed. Antibodies were immunoprecipitated with protein-G agarose, separated by SDS-PAGE, and detected by Coomassie blue staining. The arrowhead indicates the position of the antibody heavy chain. The position and relative molecular weight of marker proteins are indicated on the left. (B) Detection of anti-MDU antibody-immunoprecipitated recombinant MDU in Sf9 cells on Western blot analysis. Whole cell extracts were prepared from Sf9 cells and Sf9 cells that had been infected with a recombinant Baculo virus expressing recombinant FLAG-epitope tagged $\triangle N M D U$. Proteins from both extracts were immunoprecipitated by use of protein-G bound antiMDU antibody (with rat serum as control), separated by SDS-PAGE, and electrophoretically transferred to PVDF membrane. The Western blots were probed with anti-FLAG antibody and developed as described. The asterisk indicates the position of FLAG- $\triangle$ NMDU.

\subsubsection{Anti-5-methyl cytosine antibody}

To assess the DNA methylation and its regulation by MDU in Drosophila embryo or Drosophila Schneider S2 cells by XChIP, a anti-5-methyl cytosine antibody was purchased from Megabase (2.1.4).

To assess the association of the antibody with methylated DNA, DNA pull-down assays were performed (2.2.4). Protein-A beads were used to immobilize anti-5-methyl cytosine antibody. Rabbit serum was used as a control. The antibody-agarose complexes were incubated 
with DNA oligonuclotides containing three methylated CCA-motifs or unmethylated control DNA oligonucleotides (Figure 9).

The anti-5-methyl cytosine antibody retained methylated DNA but not unmethylated control, whereas neither DNA was retained by rabbit serum. The results indicate that anti-5methyl cytosine antibody specifically recognizes methylated cytosine residues. This antibody was used in all XChIP assays for DNA methylation detection in fly embryo or S2 cells (see 3.6.1 and 3.6.3).

A B

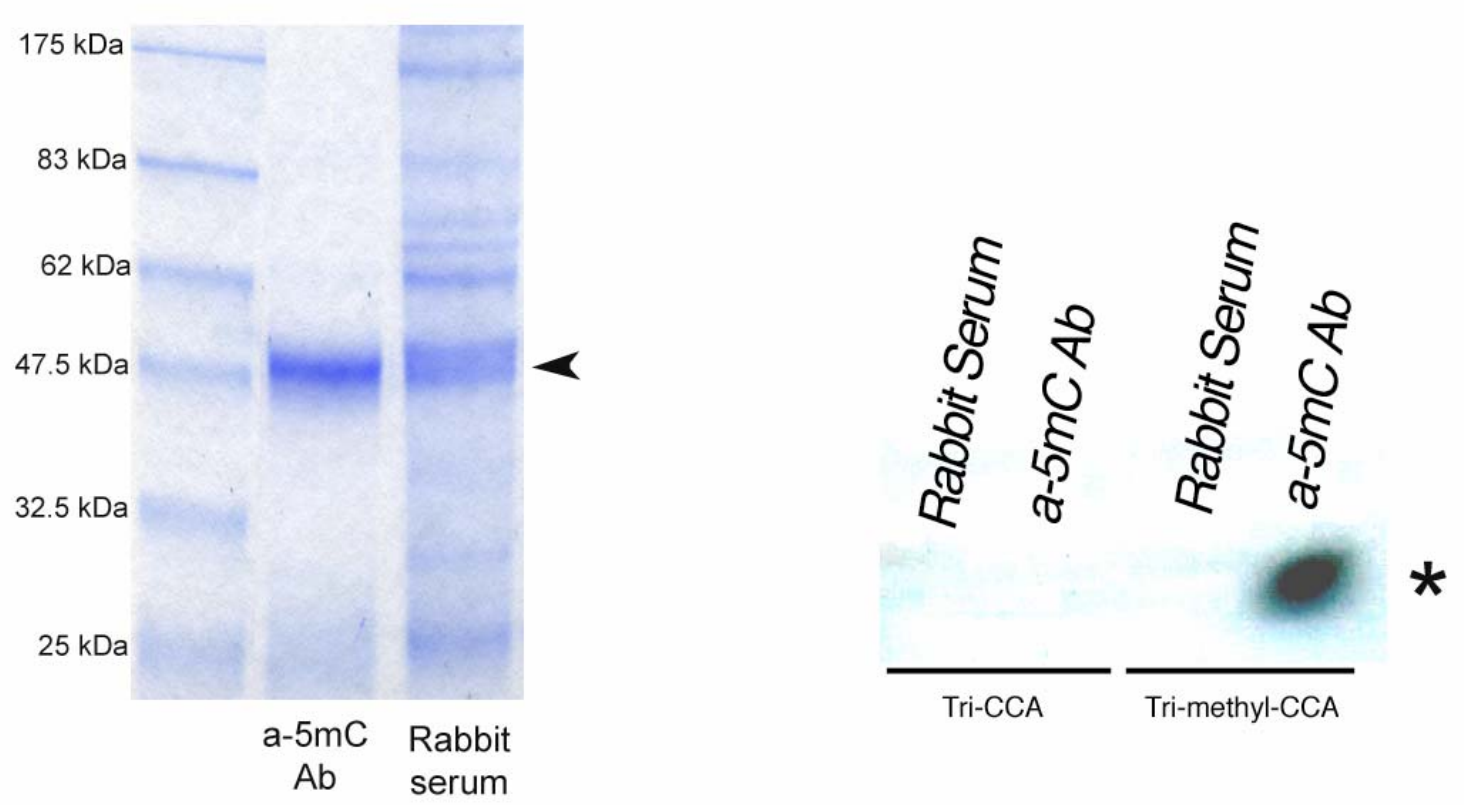

Figure 9. Functional characterization of polyclonal antibody recognizing 5-methyl cytosine. (A) Coomassie blue-stained polyacrylamide gel showing antibodies immunoprecipitated from rabbit serum and anti-5-methyl cytosine antibody (a-5mC $A b)$. Antibodies were immunoprecipitated with protein-A agarose, separated by SDS-PAGE, and detected by Coomassie blue staining. The arrowhead indicates the position of the antibody heavy chain. The position and relative molecular weight of marker proteins are indicated to the left. (B) Autoradiogram of DNA pull-down assays. 54-bp radiolabeled DNA oligonucleotides (2.1.8.1) containing 3 unmethylated (CCA) or methylated (C5mCA) motif were precipitated with antibody to 5-methyl cytosine $(5 \mathrm{mC})$ or rabbit antiserum. Precipitated DNA was separated on native polyacrylamide gels and detected by autoradiography. The asterisk indicates the position of DNA oligonucleotides. 


\subsubsection{Anti-dDNMT2 antibody}

dDNMT2 is the Drosophila DNA methyltransferase (Kunert et al., 2003). To determine the role of dDNMT2 in MDU-mediated transcriptional regulation, anti-dDNMT2 antibodies were developed at Biosynthesis by immunizing two rabbits with polypeptides $\mathrm{NH}_{2}$ GCQPHTRQGLQRDTEDKRSDAL-OH coresponding to amino acids 64-84 of dDNMT2 (2.2.8.1). The antibodies were tested for the ability to precipitate recombinant FLAG-dDNMT2 from the SF9 cell extract. Cell extract prepared from Sf9 cells expressing FLAG-dDNMT2 or Sf9 cell extract were immunoprecipitated with anti-dDNMT2 antibodies, and the precipitated proteins were detected by Western Blot using anti-FLAG antibody (Figure 10).

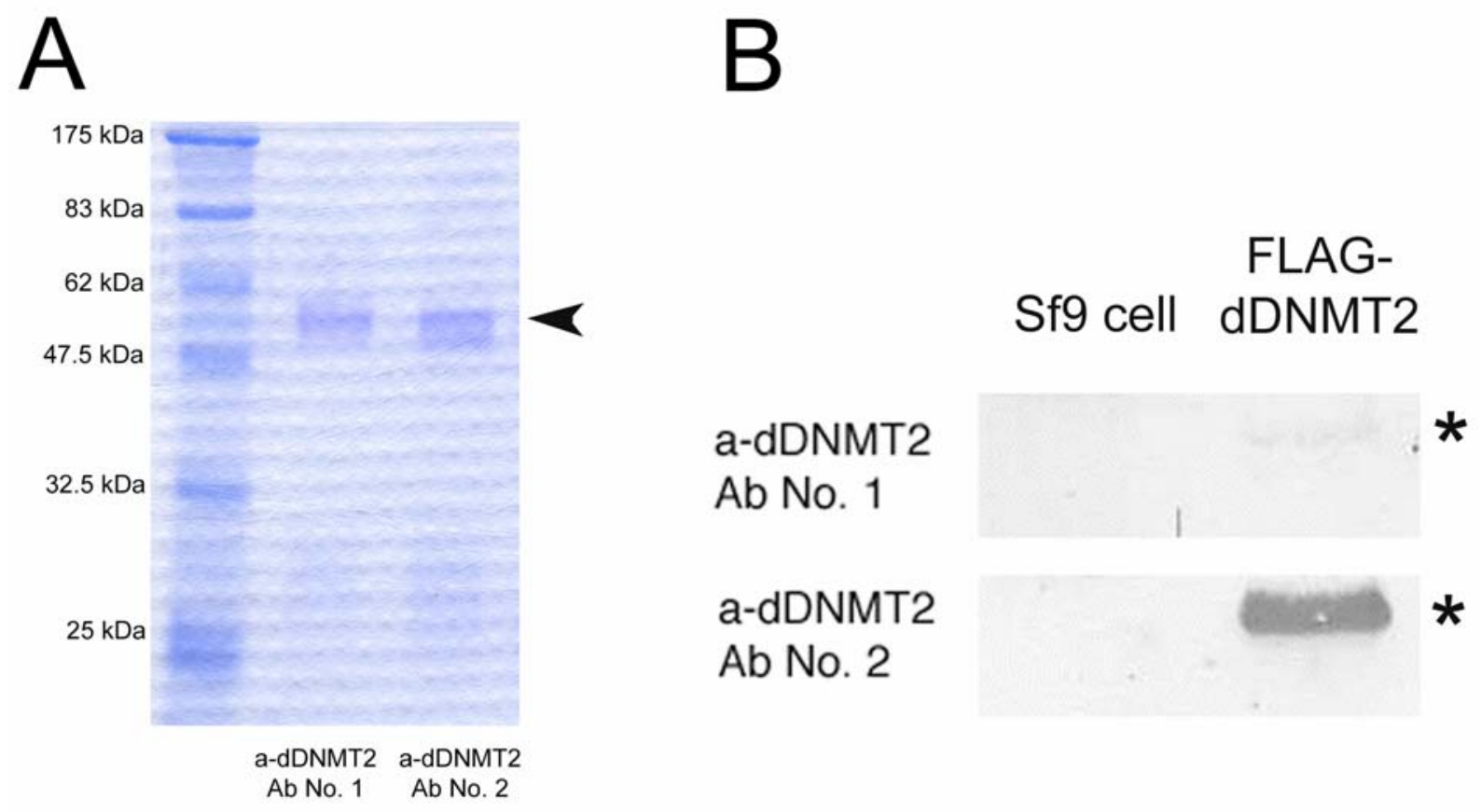

Figure 10. Functional characterization of polyclonal antibodies recognizing dDNMT2. (A) Coomassie blue-stained polyacrylamide gel showing antibodies immunoprecipitated from rabbit serum. Rabbit serum producing antibodies to dDNMT2 (a-dDNMT2 Ab No.1 or a-dDNMT2 Ab No. 2) was harvested at week 8 . Antibodies were immunoprecipitated with protein-A agarose, separated by SDS-PAGE, and detected by Coomassie blue staining. The arrowhead indicates the position of the antibody heavy chain. The position and relative molecular weight of marker proteins are indicated to the left. (B) Detection of anti-dDNMT2 antibody-immunoprecipitated recombinant dDNMT2 in Sf9 cells on Western blot analysis. Whole cell extracts were prepared from Sf9 cells and Sf9 cells that had been infected with a recombinant Baculo virus expressing recombinant FLAG-epitope-tagged dDNMT2. Proteins from both extracts were 
immunoprecipitated using protein-A bound anti-dDNMT2 antibodies No.1 or No.2, separated by SDS-PAGE, and electrophoretically transferred to PVDF membrane. The Western blots were probed with anti-FLAG antibody and developed as described. The asterisks indicate the position of FLAG-dDNMT2.

Anti-dDNMT2 antibody No.2 specifically precipitated recombinant dDNMT2, whereas little recombinant dDNMT2 was precipitated by anti-dDNMT2 antibody No.1 (Figure 10). The antidDNMT2 antibody No.2 was used for all further assays (see 3.6.1 and 3.6.3). 


\subsection{Expression of MDU in Drosophila embryo}

Recent studies suggest that MDU is expressed throughout Drosophila development (MoraBermúdez 2002; Stabell et al., 2006; Seum et al., 2007; Tzeng et al., 2007; Clough et al., 2007; Yoon 2008). To confirm the expression of MDU in Drosophila embryos, MDU was immunoprecipitated from nuclear extract prepared from 0- to 12-h-old embryos and Drosophila Schneider S2 cells (Figure 11).

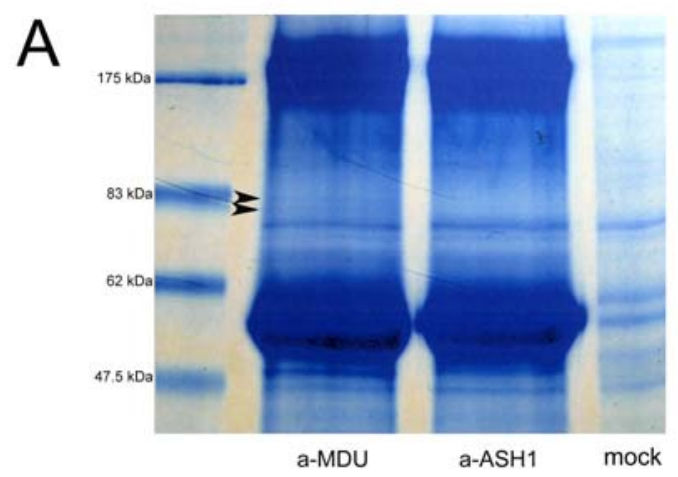

C

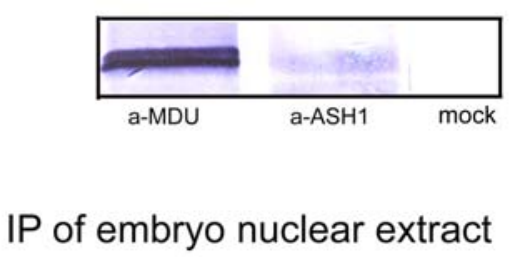

B

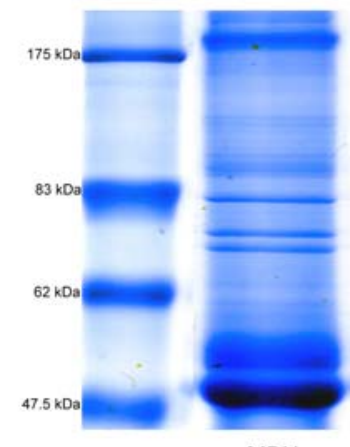

a-MDU

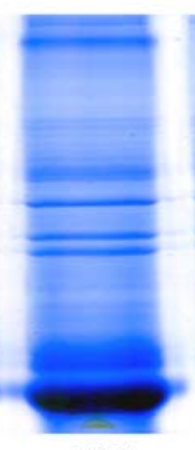

a-ASH1

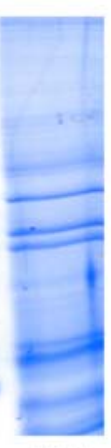

mock

D

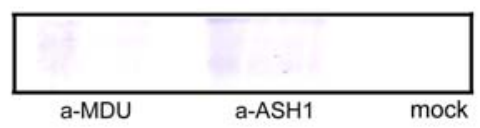

IP of S2 cell extract

Figure 11. MDU is expressed in Drosophila embryos but not in embryonic Schneider S2 cells. (A, B) Coomassie blue-stained SDS-polyacrylamide gels showing proteins precipitated without antibody (mock) or with antibody to MDU or ASH1 (top panels) from nuclear extracts prepared from 0 to 12-h-old embryos (A) or whole cell extract prepared from S2 cells (B). Precipitated proteins and antibody-protein-G complexes used for precipitation were separated by SDS-PAGE and detected by Coomassie blue staining. The arrowheads mark the position of MDU-containing protein bands. The positions and molecular weight of marker proteins are indicated to the left. (C, D) Western blot analysis of precipitated proteins. Proteins were precipitated as described in (A, B), separated by SDS-PAGE, and electrophoretically transferred to PVDF membrane. Blots were probed with antibody to MDU and developed as described (2.2.2.4). 
Extracts were precipitated with monoclonal antibodies to MDU (see 2.2.8.1) or ASH1, an epigenetic activator from Drosophila. Immunoprecipitated proteins underwent Western blot analysis (Figure 11) or mass-spectrometry (Figure 12).

On Western blot analysis, the antibody to MDU detected two proteins with a relative molecular weight of $80 \mathrm{kDa}$ in nuclear extracts of embryos but not in S2 cells. With anti-ASH1 antibody, the two proteins were not detected in embryo nuclear extract or in S2 cells (Figure 11).

The results suggest that MDU is expressed in the Drosophila embryo and is localized to the nucleus but is not expressed in $\mathrm{S} 2$ cells.

\begin{tabular}{|c|c|c|c|c|c|c|c|c|c|c|c|}
\hline \multicolumn{2}{|c|}{ Query } & Observed & $\operatorname{Mr}(\exp t)$ & Mr (calc) & Delta & Miss & Score & Expect & Rank & Peptide & \multirow{2}{*}{$\begin{array}{l}\text { Location in CG30426/MDU (amino acid) } \\
\qquad 437-443\end{array}$} \\
\hline v & $\underline{25}$ & 406.22 & 810.43 & 810.43 & -0.00 & 0 & 29 & 3 & 1 & SLAEVHR & \\
\hline V & $\underline{117}$ & 684.37 & 1366.73 & 1366.71 & 0.01 & 0 & 21 & 15 & 1 & KPIEEIGYQYK & $557-567$ \\
\hline V & $\underline{133}$ & 739.87 & 1477.72 & 1477.71 & 0.01 & 0 & 39 & 0.21 & 1 & TNAGGVSTSNSASAVR & 333-348 \\
\hline 证 & $\underline{143}$ & 516.94 & 1547.80 & 1547.80 & -0.01 & 0 & 11 & $1.4 \mathrm{e}+02$ & 1 & FPWVAFFSAAHIR & $795-807$ \\
\hline$\square$ & $\underline{161}$ & 894.46 & 1786.90 & 1786.88 & 0.01 & 0 & 33 & 1.2 & 1 & AVINFNPNADLDETVR & $729-744$ \\
\hline & $\underline{174}$ & 638.98 & 1913.92 & 1913.92 & -0.01 & 0 & (2) & $9.2 \mathrm{e}+02$ & 8 & HLNNSTIYVDDENRPK & $349-364$ \\
\hline 口 & $\underline{175}$ & 638.98 & 1913.93 & 1913.92 & 0.00 & 0 & 13 & 69 & 1 & HLNNSTIYVDDENRPK & $349-364$ \\
\hline$\square$ & $\underline{177}$ & 644.68 & 1931.01 & 1931.02 & -0.01 & 0 & 44 & 0.062 & 1 & LDSYSPLAKPLLSGWER & $398-414$ \\
\hline 田 & 183 & 1014.45 & 2026.89 & 2026.87 & 0.02 & 0 & 62 & 0.00079 & 1 & SGSTQNSSTQSSELDSQER & $710-728$ \\
\hline v & $\underline{184}$ & 684.67 & 2050.99 & 2050.98 & 0.01 & 0 & (28) & 1.9 & 1 & ATENVLNVDNFDFTPDLK & $447-464$ \\
\hline v & $\underline{185}$ & 1026.51 & 2051.00 & 2050.98 & 0.02 & 0 & 56 & 0.0033 & 1 & ATENVLNVDNFDFTPDLK & $447-464$ \\
\hline$\nabla$ & $\underline{222}$ & 791.73 & 2372.18 & 2372.17 & 0.01 & 1 & 19 & 13 & 1 & AVINFNPNADLDETVRENSVR & $729-749$ \\
\hline
\end{tabular}

Figure 12. Mass-spectrometry analyses of immunoprecipitated MDU. Schematic representation of mass-spectrometry analysis of the two protein bands reacting with antibody to MDU (see Figure 11). The figure shows the expected and calculated relative molecular weight and the sequence of peptides corresponding to MDU.

Mass-spectrometry analysis of the two proteins confirmed that they are expressed by Mdu (CG30426) (Figure 12). The expected molecular weight of MDU is $95.48 \mathrm{kDa}$. Thus, the relative molecular weight of the two proteins precipitated by anti-MDU antibody is smaller than that predicted. This finding suggests that the transcript of MDU may undergo alternative splicing. Also, post-translational modifications might have contributed to the difference in protein molecular weight between predicted and real MDU. 


\subsection{The MBD of MDU binds methylated DNA}

MDU contains an MBD domain with an unknown function. Recently, one study demonstrated that Drosophila MBD protein dMBD2/3 binds methylated CpT/A motifs in vitro (Marhold et al., 2004), which raises the possibility that the MBD of MDU binds methylated DNA as well. To test this, I performed in vitro DNA pull-down assays. Recombinant MBD of MDU fused to the GST, or GST alone, was immobilized in glutathione agarose (Figure 13) and incubated with radiolabeled DNA containing one or three copies of a methylated CpG, CpT or CpA motif. Recombinant proteins and radiolabeled DNA were generated as described (2.2.3, 2.2.1.3 and 2.2.1.4).

\section{GST- \\ GST MBD}

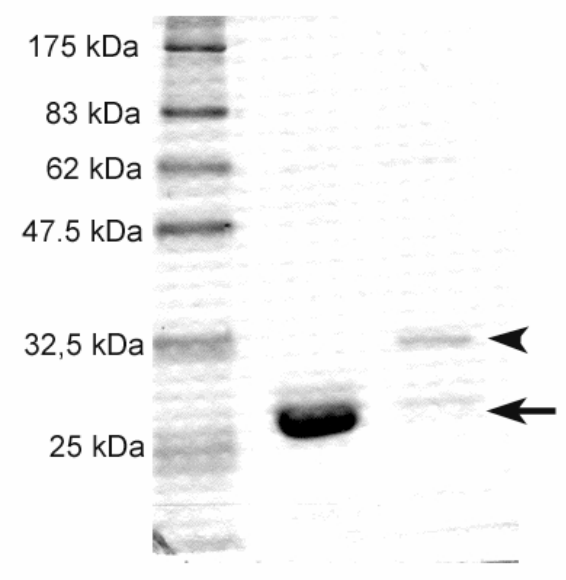

Figure 13. Coomassie blue-stained SDS-polyacrylamide gel showing affinity purified GST or GST-MBD. GST and GST-MBD were expressed in bacteria and purified by glutathione affinity chromatography as described (2.2.3.3). Purified proteins were separated by SDS-PAGE and detected by Coomassie blue staining. The arrow indicates the position of GST and the arrowhead the position of GST-MBD.

Recombinant protein and DNA were incubated as described (2.2.4.1). Retained DNA was purified, separated on native polyacrylamide gels, and detected by autoradiography (2.2.4.2). MDU preferentially retained DNA containing one or multiple methylated CpA motifs but 
did not bind other methylated CpX motifs and unmethylated DNA. The GST part of the GSTMBD fusion protein did not bind methylated or unmethylated DNA (Figure 14 and 15).

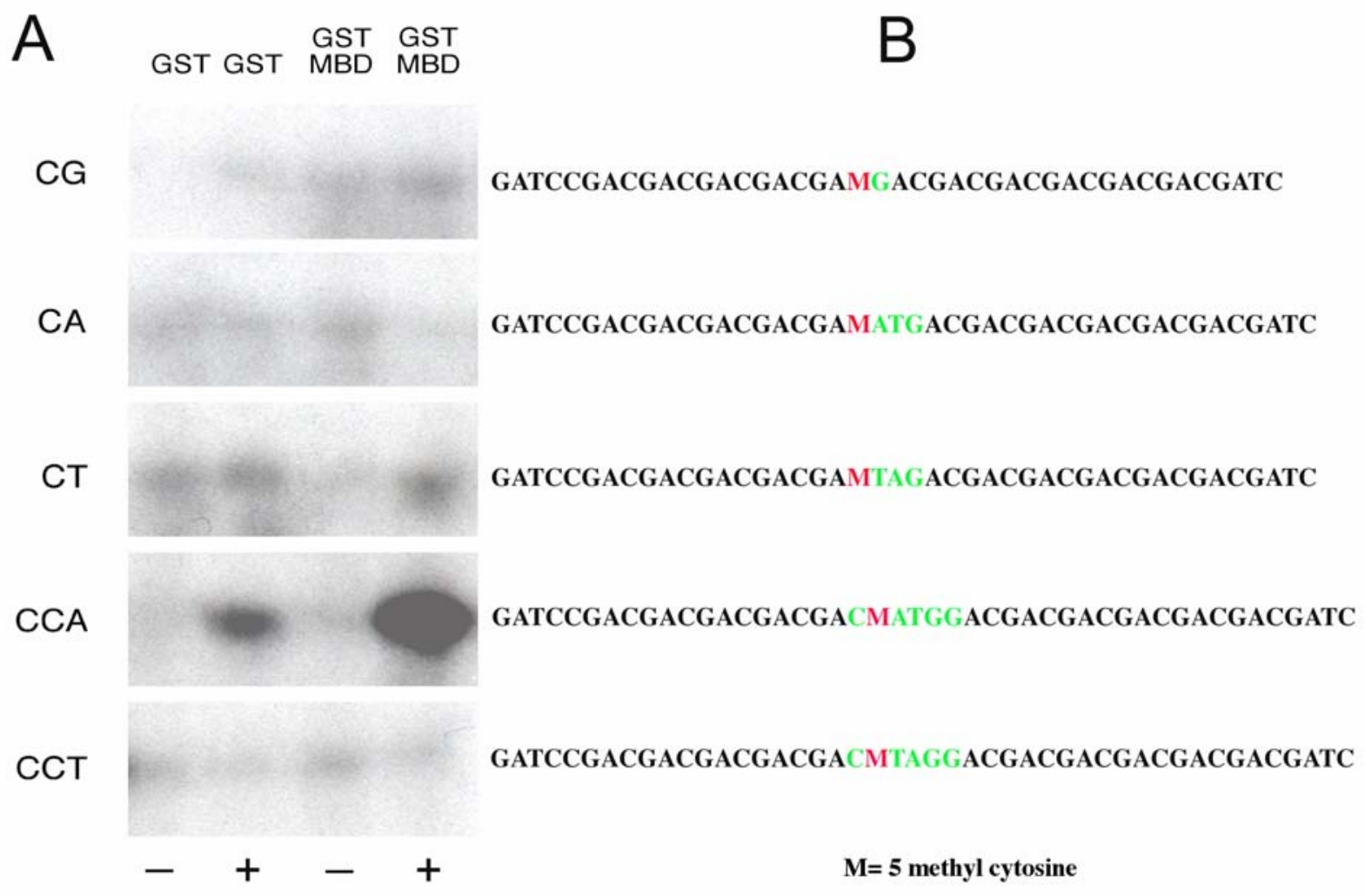

Figure 14. MDU binds methylated CpA motif. (A) Autoradiograms of DNA pull-down assays using GST, GST-MBD (see Figure 13), and radiolabeled methylated (+) and unmethylated (-) DNA oligonucleotides, whose sequence is indicated in (B). GST and GST-MBD were incubated with radiolabeled DNA. Retained DNA was separated on native polyacrylamide gels and detected by autoradiography. The sequence of the methylated core-motif is indicated to the left. (B) Sequence of the DNA oligonucleotides used for DNA pull-down assays described in (2.1.8.1). The sequence of the core-moif is indicated in green. The red $M$ represents 5-methyl cytosine. 

A
GST GST
GST GST MBD MBD
Tri-CG
GATCCGACGACGACGACGAMGAMGAMGAC
GACGACGACGACGACGATC
Tri-CA

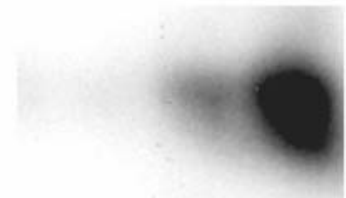
GATCCGACGACGACGACGAMATGAMATGAMATGAC
GACGACGACGACGACGATC
Tri-CT
GATCCGACGACGACGACGAMTAGAMTAGAMTAGAC
GACGACGACGACGACGATC
Tri-CCA
GATCCGACGACGACGACGACMATGGACMATGGACMATGG ACGACGACGACGACGACGATC
Tri-CCT
GATCCGACGACGACGACGACMTAGGACMTAGGACMTAGG ACGACGACGACGACGACGATC

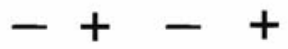
M = 5 methyl cytosine

Figure 15. MDU binds DNA containing multiple methylated CpA motifs. (A) Autoradiograms of DNA-pull down assays were performed as described (2.2.4) except that DNA oligonucleotides containing three methylated core-motifs were used. The sequence of the DNA oligonucleotides is described in (B). The methylated core-motifs are indicated in green. The red M represents 5methyl cytosine.

Collectively, these results suggest that the MBD domain of MDU specifically binds methylated $\mathrm{CpA}$ motifs. The results correspond well with the result that Drosophila genome predominantly contains CpA or CpT motifs) (Lyko et al., 2000a; Gowher et al., 2000). 


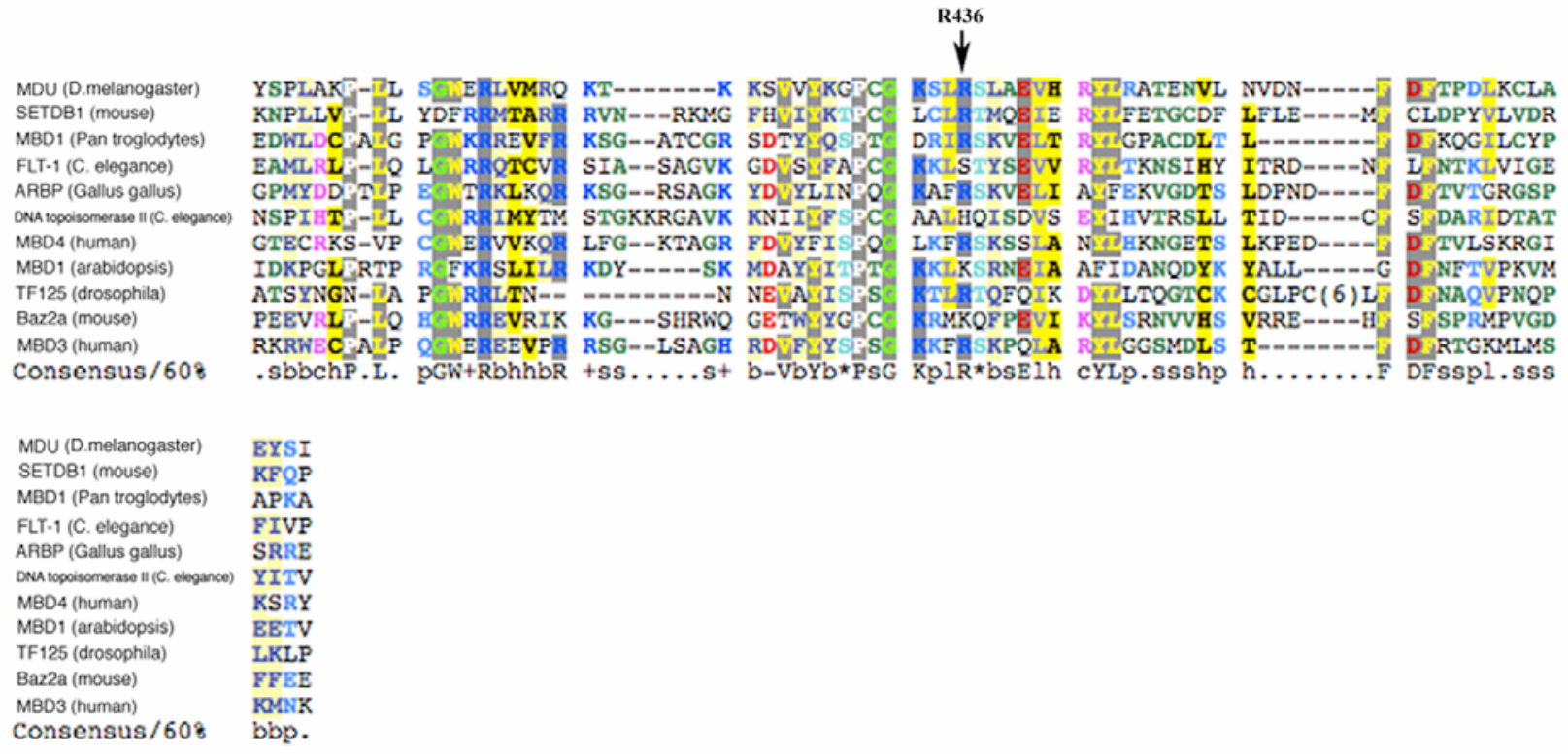

Figure 16. Computational comparison of the MBD of MDU with MBD domains from plant and animals. Sequence alignment was performed using SMART (http://smart.emblheidelberg.de/). The arrow indicates the conserved arginine 436 aa in the MBD of MDU.

Sequence comparison analysis of the MBD of MDU with the MBD of other proteins revealed that the MBD of MDU contains key amino acids involved in the interaction of the MBD of vertebrate proteins in particular MeCP2 with methylated DNA. One of the conserved key amino acids is arginine (R) 436, which corresponds to arginine 133 in MeCP2 of humans (Figure 16). The mutation of R133 to cysteine (C) attenuates the DNA binding ability of the MBD of MeCP2 (Free et al., 2001).

To confirm that the MBD of MDU binds methylated CpA motifs, DNA pull-down assays were performed as described (2.2.4) but with GST fusion proteins containing the wild-type MBD of $M D U$ or a mutant MBD-derivative $[M B D(R 436 C)]$. $M B D(R 436 C)$ contains a single amino acid exchange mutation, which replaces arginine 436 by cysteine (see 2.2.1.15). GST, GST-MBD, and GST-MBD(R436C) (Figure 17) were used in DNA pull-down assays. The probe DNA contained three methylated DNA motifs.

The results confirmed that wild-type MBD of MDU retained methylated DNA but did not bind unmethylated DNA. In contrast, $\operatorname{MBD}(\mathrm{R} 436 \mathrm{C})$ did not retain methylated or unmethylated DNA (Figure 18). The results suggest that the conserved arginine of the MBD domain of MDU is responsible for its methylated DNA binding activity. 


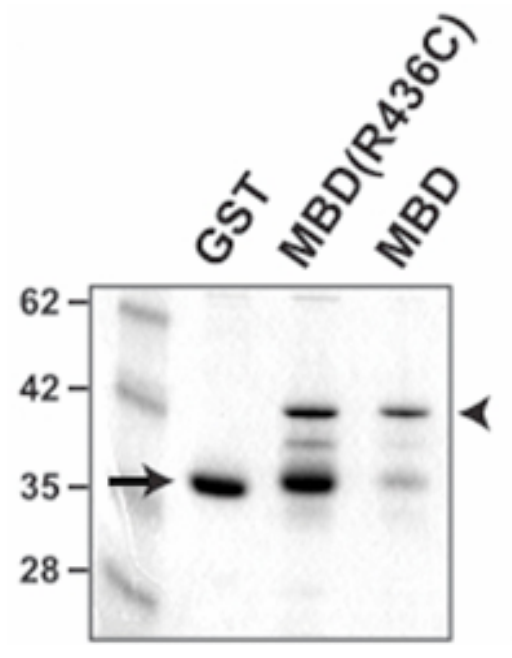

Figure 17. Purification of GST-MBD fusion proteins. Coomassie blue-stained SDSpolyacrylamide gel showing affinity-purified GST, GST-MBD, and GST-MBD(R436C). Proteins were expressed in bacteria and purified by glutathione affinity chromatography (see 2.2.3.3). Purified proteins were separated by SDS-PAGE and detected by Coomassie blue staining. The arrowhead indicate the position of GST-MBD fusion proteins, the arrow marks the position of GST. The position and relative molecular weight of marker proteins is indicated to the left.

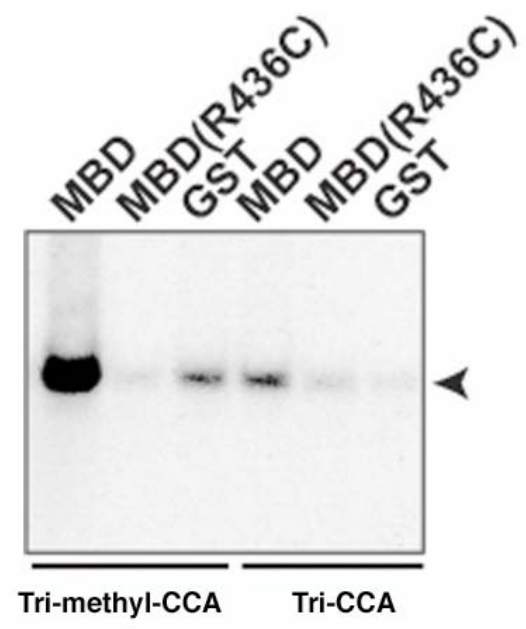

Figure 18. The point mutation R436C attenuates the interaction of the MBD of MDU with methylated DNA. Autoradiogram of DNA pull-down assays with GST, GST-MBD, and GST$\mathrm{MBD}(\mathrm{R} 436 \mathrm{C})$ and DNA oligonucleotides containing three methylated (tri-methyl-CCA) or unmethylated (Tri-CCA) CCA-motifs. DNA pull-down assays were performed as described (2.2.4). The arrowhead indicates the position of the radiolabeled DNA. 


\subsection{Histone methyltransferase activity of MDU}

\subsubsection{The SET domain of MDU methylates H3K9}

MDU contains a bifurcate SET domain (aa601-823) flanked by a Pre-SET motif (amino acid 478585) and a Post-SET motif (amino acid 826-842). Protein alignment of SET-domains with use of the on-line protein database SMART (http://smart.embl-heidelberg.de/) revealed that the NHSC motif of other SET domain HMTs is also preserved in MDU (Figure 19). In HMTs, like human Suv39h1 and Drosophila Su(var)3-9, the SET domain, together with the Pre- and Post-SET motifs, confer HMT activity. This activity depends on an intact NHSC motif, as mutations of the histidine residue of the NHSC-motif of Suv39h1 and Ash1 attenuates the HMT activity of the enzymes (Rea et al., 2000; Beisel et al., 2002).

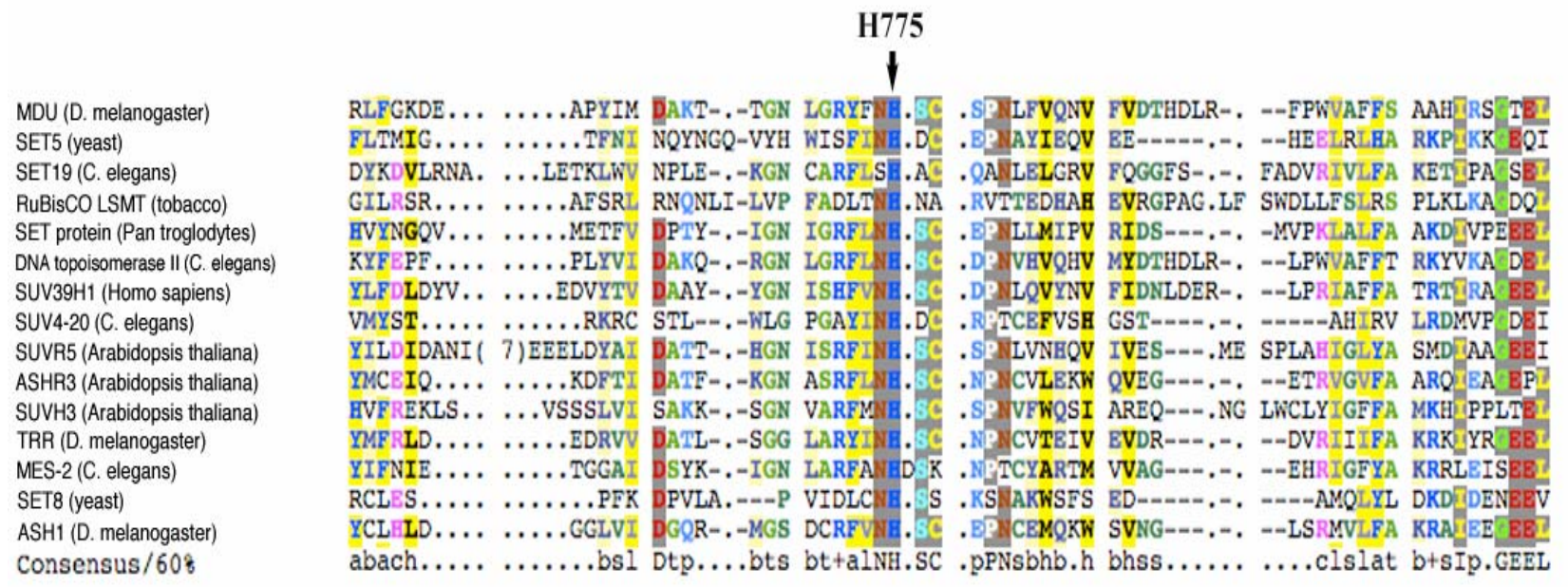

Figure 19. Comparison of SET domain of MDU (aa 750-812) with the SET domains of plant and animal proteins. Alignment involved use of SMART (http://smart.embl-heidelberg.de/). The arrow indicates the position of the conserved histidine residue (histidine 775 in MDU) in the conserved NHSC motif. 
Recent in vitro and in vivo studies demonstrated that MDU methylates H3K9 (MoraBermúdez, 2002; Stabell et al., 2006; Seum et al., 2007; Tzeng et al., 2007; Clough et al., 2007; Yoon 2008). To confirm that MDU has intrinsic HMT activity and to decipher the role of SET domain in the histone methylation activity of MDU, in vitro HMT assays using wild-type or mutant derivatives of MDU were performed. Recombinant histone $\mathrm{H} 3$ was incubated with radiolabled $\left[{ }^{3} \mathrm{H}\right]$-S-adenosylmethionine (SAM) and either $\Delta \mathrm{N}-\mathrm{MDU}$ (amino acids 246-842) or mutated $\Delta \mathrm{N}$ MDU(H775L), which contains a single exchange mutation which replaces histodine-residue 775 by leucine. Histidine 775 is part of the conserved NHSC motif that is essential for the HMT activity of HMTs (Rea et al., 2000).

After incubation, reaction products were separated by SDS-PAGE and detected by fluorography. MDU methylated $\mathrm{H} 3$ but not other histones. In contrast, the MDU mutant $(\mathrm{MDU}(\mathrm{H} 775 \mathrm{~L}))$ did not methylate H3 (Figure 20). The result suggested that the HMTase activity is intrinsic to MDU. The SET domain of MDU, with conserved functional motifs (e.g., NHSC), is responsible for this activity.

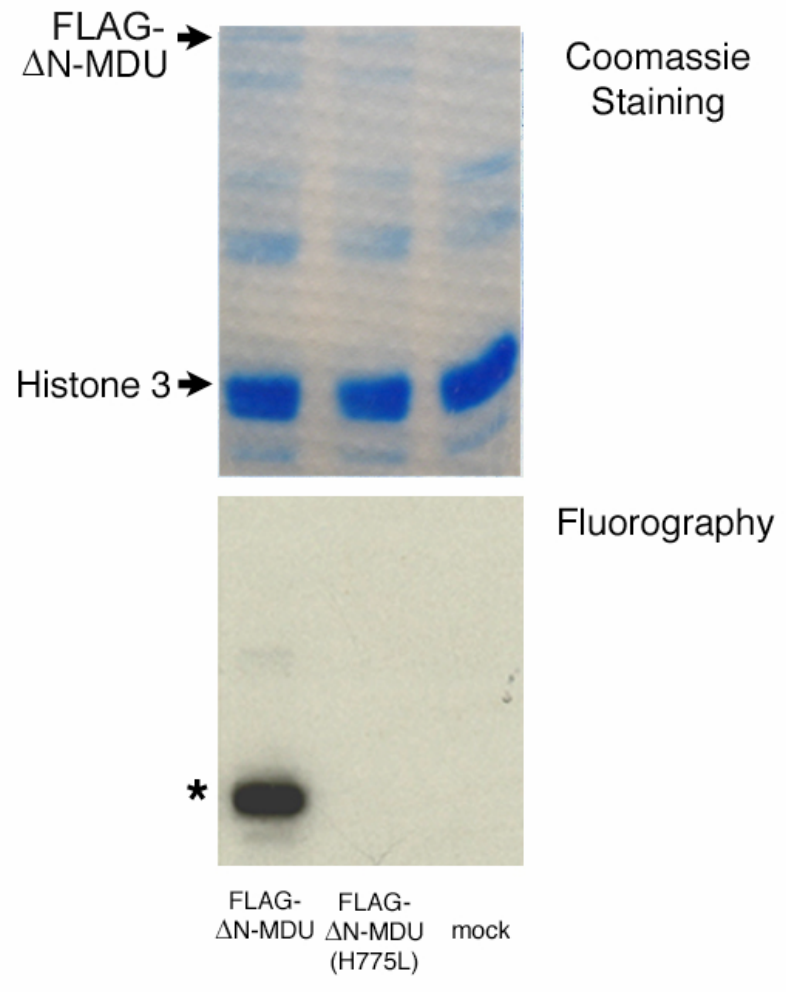

Figure 20. MDU has intrinsic HMT activity. Coomassie blue-stained SDSpolyacrylamide gel (top) and corresponding fluorogram (bottom) of HMT assays programmed with recombinant histone $\mathrm{H} 3$, radiolabeled S-adenosylmethionine (SAM) and $\triangle \mathrm{N}-\mathrm{MDU}, \quad \Delta \mathrm{N}-\mathrm{MDU}(\mathrm{H} 775 \mathrm{~L})$, or no protein (mock). Reaction products were separated by SDS-PAGE and radiolabeled proteins were detected by fluorography (see 2.2.6). The positions of methylated histone 3 and FLAG- $\triangle N-M D U$ are indicated. The asterisk indicates the position of radiolabeled $\mathrm{H} 3$. 


\subsubsection{MDU tri-methylates lysine 9 in histone H3}

The $\varepsilon$-amino group of lysine residue can accept one, two, or three methyl groups to form mono-, di-, or tri-methylated products, respectively. Different HMTs have a different activity concerning the number of methyl groups (one, two, or three) they transfer to a target lysine. For example, the S. cerevisiae SET1 protein can catalyze di- and trimethylation of H3 Lysine 4 (Santos-Rosa et al., 2002). In contrast, human SET7/9 protein generates exclusively mono-methyl Lysine 4 of H3 (Kwon et al., 2003 and Xiao et al., 2003b). Furthermore, DIM-5 of Neurospora crassa generates tri-methyl Lysine 9 (Tamaru et al., 2003).

To investigate whether MDU mono-, di-, or tri-methylates H3K9, HMT-assays were performed (Figure 21A). The reaction products were separated by SDS-PAGE and electrophoretically transferred onto PVDF membrane. The reactions products were detected by Western blot analysis with antibodies recognizing mono-, di-, or tri-methylated H3K9 (Abcam 1:100000, see 2.1.4) (Figure 21B).

A

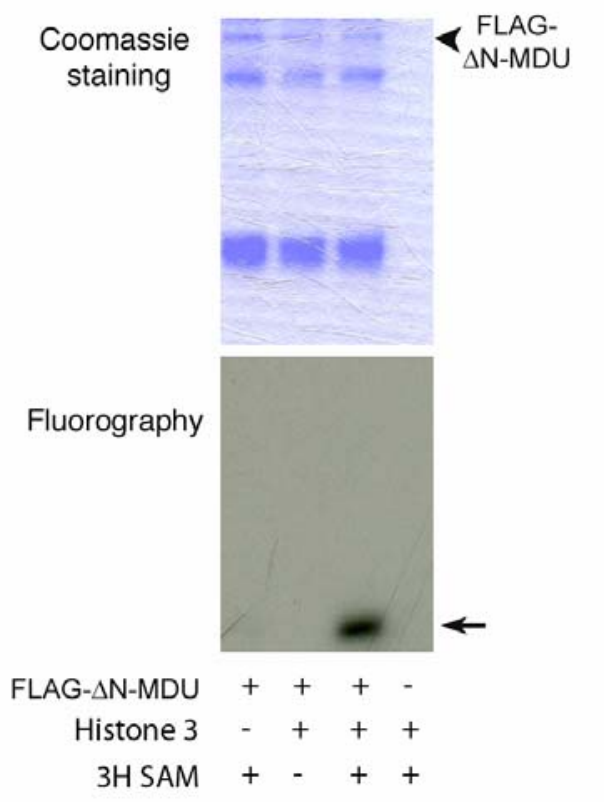

B a-Mono Me H3K9 a-Di Me H3K9 a-Tri Me H3 K9
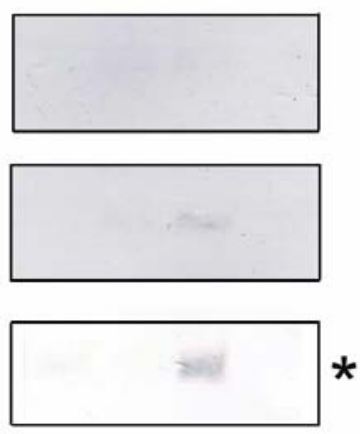

FLAG- $\triangle N-M D U$

Histone $3-+++$ 3H SAM +-++

Figure 21. MDU tri-methylates histone H3. (A) Coomassie blue-stained SDS-polyacrylamide gel (A, top) and corresponding fluorogram (A, bottom) of HMT assays programmed with various combinations of recombinant histone $\mathrm{H} 3$, radiolabeled S-adenosylmethionine (SAM) and $\Delta \mathrm{N}$ - 
MDU. HMT assays were performed as described (2.2.6.1). Reaction products were detected by fluorography (2.2.6.2). The arrow indicates the position of radiolabeled H3. (B) Western blot analysis of the reaction products of HMT assays described in (A). Reaction products were separated by SDS-PAGE and electrophoretically transferred to PVDF membranes. The blots were probed with antibodies recognizing mono-, di-, or tri-methylated $\mathrm{H} 3 \mathrm{~K} 9$ (a-mono me H3K9, a-di me $\mathrm{H} 3 \mathrm{~K} 9$, a-tri me $\mathrm{H} 3 \mathrm{~K} 9$ as indicated to the left of the 3 panels in (B)). Western blots were developed as described (2.2.2.4)

On Western blot analysis, the antibody to tri-methyl $\mathrm{H} 3 \mathrm{~K} 9$ recognized a band corresponding to $\mathrm{H} 3$. In contrast, mono- and di-methylation of $\mathrm{H} 3 \mathrm{~K} 9$ was undetectable, which suggests that MDU preferentially methylates $\mathrm{H} 3 \mathrm{~K} 9$ in vitro. Tri-methylation of $\mathrm{H} 3 \mathrm{~K} 9$ is a hallmark of gene silencing (Richards \& Elgin 2002; Grewal \& Moazed 2003; Sims et al., 2003). Thus, MDU could be a transcription repressor whose function in gene silencing involves H3K9-specific HMT activity. 


\subsection{MDU-mediated methylation of H3K9 mediates gene silencing}

\subsubsection{MDU-mediated repression demonstrated on luciferase assay}

Tri-methylation of $\mathrm{H} 3 \mathrm{~K} 9$ has been associated with gene silencing in eu- and heterochromatin (Richards \& Elgin 2002; Grewal \& Moazed 2003; Sims et al., 2003). The ability of MDU to trimethylate $\mathrm{H} 3 \mathrm{~K} 9$ strongly supported the hypothesis that MDU acts as a repressor of transcription.

To test this hypothesis, transfection assays were used to investigate the transcriptional regulatory potential of MDU in Drosophila Schneider S2 cells. S2 cells are a suitable test system for the analysis of MDU-dependent transcriptional regulation, because S2 cells do not express MDU, which allows for the analysis of exogenous wild type and mutant MDU derivatives in transcriptional regulation.

MDU does not contain a "classical" DNA binding domain. In addition, at the time of these experiments, target genes for MDU were unknown. To circumvent those problems, I designed a transcription test system for MDU in S2 cells that is based on the TET-on/off system. The TETon/off system uses the bacterial tetracycline repressor (TetR) and TetR-dependent reporter genes to investigate the transcriptional regulatory potential of eukaryotic transcription factors (Gossen and Bujard 1992; Anastassiadis et al., 2002) To tether MDU to a TetR-dependent reporter gene, we generated plasmids expressing fusion proteins consisting of TetR and wildtype or mutant $\mathrm{MDU}(\mathrm{H} 775 \mathrm{~L})$ (which lacks HMT-activity in vitro (3.4.1)). To investigate the transcriptional potential of MDU in chromatin, a stable S2 cell line--S2-tetO-tk-luc (2.1.7) was generated that contains the TetR-dependent reporter gene (tetO-tk-luc). The reporter contains 6 copies of the tet-operator (the target DNA for TetR) fused to a thymidine kinase minimal promoter, which drives the expression of the reporter gene firefly luciferase (luc). To monitor repression by MDU, we selected a S2-tetO-tk-luc cell line that supports a high basal level of luc expression (Figure 22). 


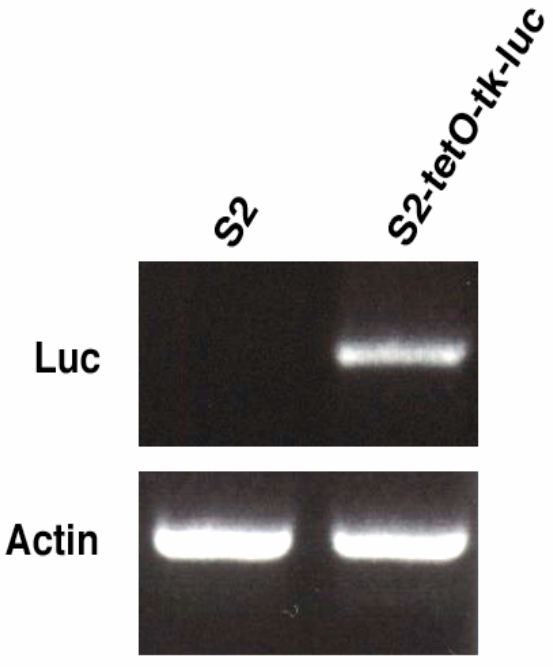

Figure 22. High basal transcriptional activity of the luciferase reporter gene in S2-tetO-tk-luc cells. Ethidium bromide-stained agarose gel showing the reaction products of $\mathrm{PCR}$ assays detecting the transcription of actin and luciferase (Luc) in S2 cells and S2-tetO-tk-luc cells. RNA was isolated, reverse transcribed and analyzed as described (2.2.1.2.3).

S2-tetO-tk-luc cells were co-transfected with a plasmid expressing green fluorescent protein (GFP) and plasmids expressing TetR fused to MDU or MDU(H775L). FACS isolated GFP expressing cells (Figure 23), which coexpress TetR-MDU or TetR-MDU(H775L) fusion proteins. Reporter gene expression was determined as described (2.2.7).
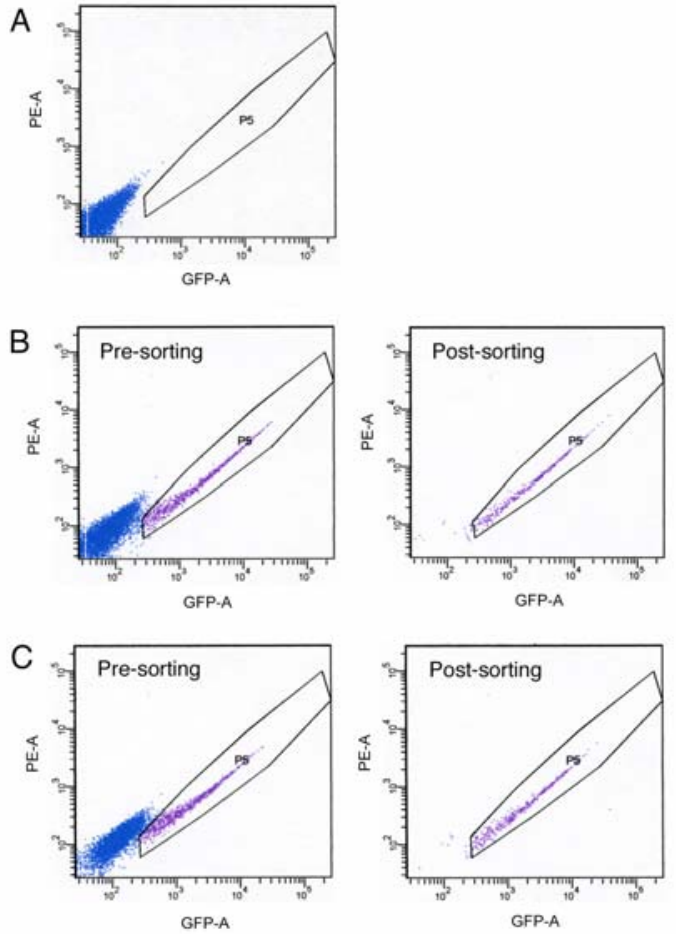

Figure 23. Schematic representation of FACS analysis. FACS isolated GFP-expressing cells from (A) S2-tetO-tk-luc cells, (B) S2-tetO-tk-luc cells transfected with pAcGFP and TetR-MDU or (C) S2tetO-tk-luc cells transfected with pAcGFP and TetR$\mathrm{MDU}(\mathrm{H} 775 \mathrm{~L})$. The relative amount of GFP-positive and -negative cells before sorting and after sorting are shown for each group of cells. P5 indicates the pool of GFP positive cells. 
Cells expressing TetR-MDU showed significantly lower reporter gene expression than untransfected S2-tetO-tk-luc cells. In contrast, reporter gene activity was not significantly reduced in cells expressing TetR-MDU(H775L) (Figure 24). Collectively, the results indicate that MDU acts as a repressor of gene expression, and MDU-mediated repression involves the HMT activity of the SET domain.

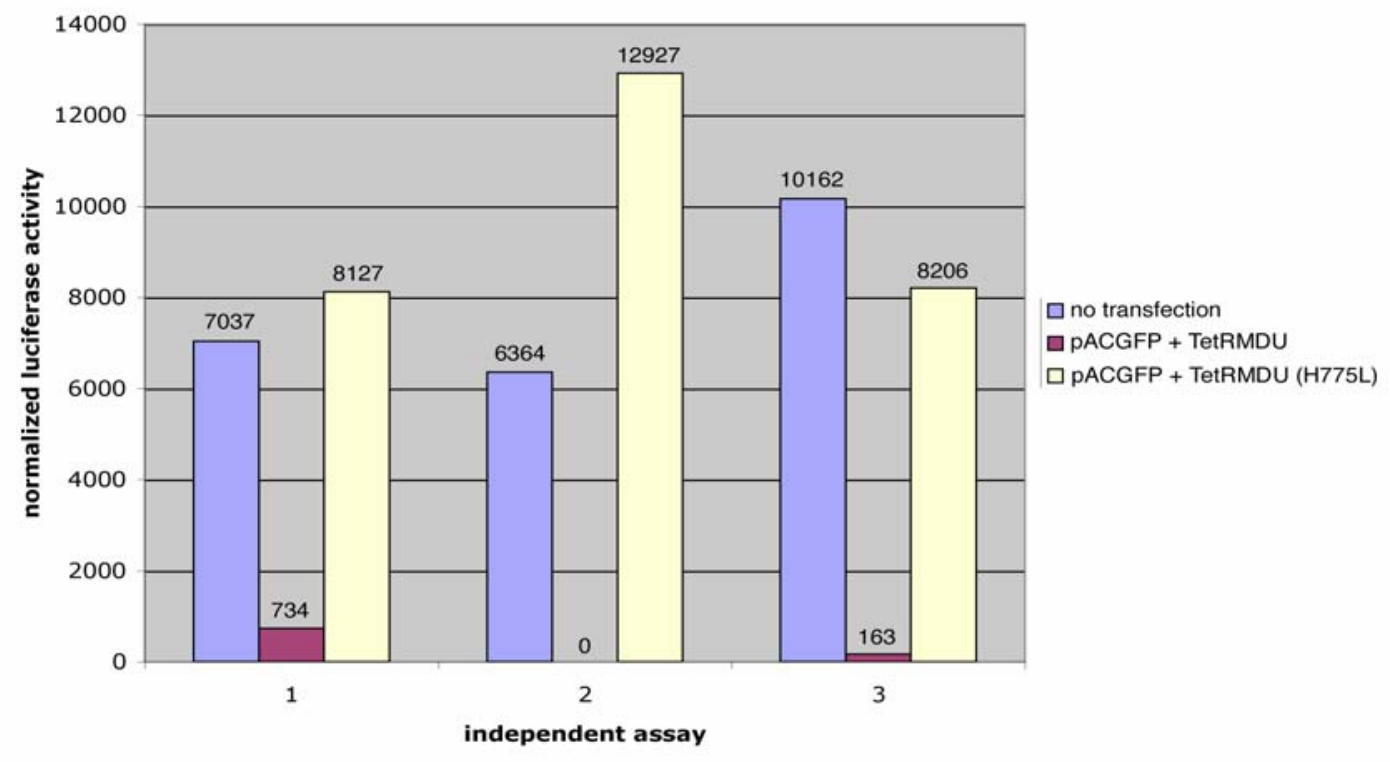

Figure 24. The SET domain of MDU is involved in transcriptional repression. Schematic representation of luciferase reporter gene assays. Luciferase activity was determined in whole cell extracts of S2-tetO-tk-luc cells and in S2-tetO-tk-luc cells transiently expressing GFP and tetR-MDU or tetR-MDU(H775L). After co-transfection, FACS isolated GFP-positive Luciferase activity was measured as described (2.2.7.4). Results of 3 assays are shown.

\subsubsection{MDU-mediated methylation of $\mathrm{H} 3 \mathrm{~K} 9$ is involved in gene silencing}

The involvement of the HMT-activity of MDU in repression raised the question of whether MDUmediated repression of gene expression involves methylation of $\mathrm{H} 3 \mathrm{~K} 9$. To address this question, cross-linked chromatin immunoprecipitation (XChIP) experiments were performed (2.2.9).

In vivo cross-linked chromatin was isolated from wild-type S2-tetO-tk-luc cells and GFPpositive S2-tetO-tk-luc cells expressing TetR-MDU or TetR-MDU(H775L) (see 2.2.10.1). 
Chromatin was sheared into fragments of 700-bp average length and immunoprecipitated with antibodies to MDU and tri-methylated H3K9. DNA was isolated from immunoprecipitated chromatin and used as a template for PCR assays, which monitored the presence of the reporter gene promoter in precipitated DNA pools (see 2.2.10).

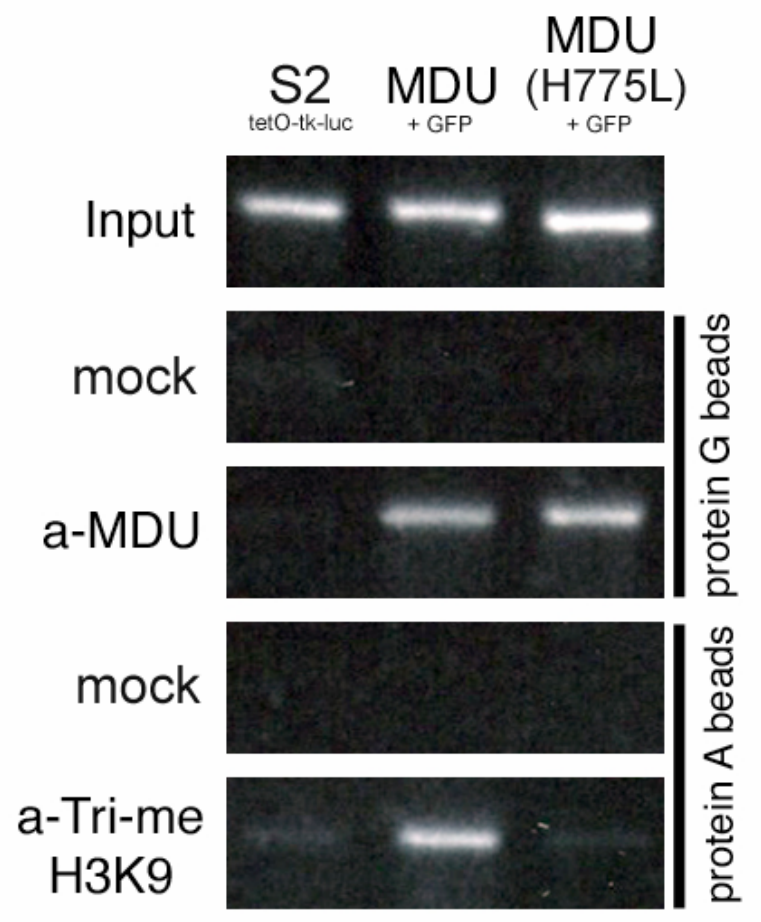

Figure 25. MDU-mediated tri-methylation of $\mathrm{H} 3 \mathrm{~K} 9$ is involved in transcriptional silencing. Ethidium bromide-stained agarose gels showing the reaction products of PCR assays detecting the promoter region of the tetO-tk-luc reporter gene in DNA pools generated by XChIP. In vivo cross-linked chromatin was isolated from S2-tetO-tk-luc cells and S2-tetO-tk-luc transiently expressing GFP and tetR-MDU or tetR-MDU(H775L) (see Figure 24)

Chromatin was precipitated using antibodies to MDU, tri-methylated $\mathrm{H} 3 \mathrm{~K} 9$, or mock precipitated (mock). PCR assays detected the promoter region of the reporter gene in DNA isolated from $1 \%$ input chromatin and in immunoprecipitated DNA pools.

MDU and tri-methyl $\mathrm{H} 3 \mathrm{~K} 9$ were not detected at the transcriptionally active reporter gene in S2-tetO-tk-luc (Figure 25). In contrast, MDU and tri-methyl H3K9 were detected at the transcriptionally inactive reporter gene promoter in cells expressing wild-type TetR-MDU (Figure 25). MDU but not tri-methyl H3K9 was present at the reporter gene in cells expressing TetR$\mathrm{MDU}(\mathrm{H} 775 \mathrm{~L})$ (Figure 25). The results indicate that repression of reporter gene expression involves tri-methylation of H3K9 by MDU. Collectively, the results support the hypothesis that MDU mediates gene silencing through methylation of $\mathrm{H} 3 \mathrm{~K} 9$. 


\subsection{MDU regulates the epigenetic repression of $R b f$}

\subsubsection{Identification of MDU target genes}

DNA methylation in Drosophila was discovered in 2000, and only very few methylated DNA sequences have been identified in the Drosophila genome (Lyko et al., 2000a; Gowher et al., 2000; Salzberg et al., 2004). Target genes for MDU are unknown. To identify target genes for MDU, XChIP assays were performed. Chromatin was isolated from 0 to 12-h-old Drosophila embryos and S2 cells and precipitated with antibodies to MDU. DNA was purified for immunoprecipitated chromatin. PCR monitored the presence of DNA sequences known to be methylated in the fly in immunoprecipitated DNA pools. These assays resulted in the identification of several putative target genes for MDU. One of the identified MDU target genes is "retinoblastoma family protein" (Rbf), the Drosophila ortholog of the human tumour suppressor gene $R b$. Rbf controls cell proliferation and cell cycle progression during Drosophila development (Du et al., 1996a; Du et al., 1996b). Repression of Rbf involves DNA hypermethylation in Drosophila (Ferres-Marco et al., 2006).

To investigate whether MDU is involved in transcriptional regulation of $R b f$ expression, I used RT-PCR and XChIP assays. For RT-PCR assays, total RNA was isolated from embryos (012-h) and $S 2$ cells and reverse transcribed to generate cDNA pools representing the transcriptome of embryos and S2 cells. PCR monitored the presence of Rbf mRNA in the generated cDNA pools (2.2.1.2.3) (Figure 26). Rbf was actively transcribed in S2 cells (Figure 26), which do not express MDU (Figure 11). In contrast, Rbf transcription was signifcantly reduced in emrbryos (Figure 26) which express MDU (Figure 11), which suggests that repression of $R b f$ coincides with the expression of MDU. 


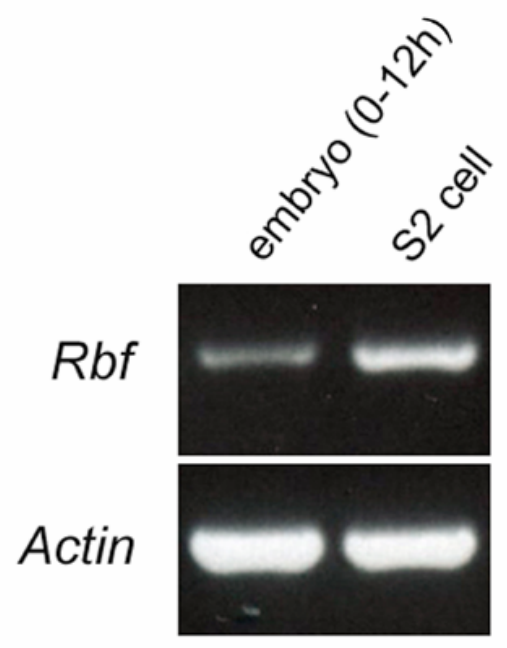

Figure 26. $R b f$ transcription in embryos and $S 2$ cells. Ethidium bromide-stained agarose gel showing the reaction products of PCR assays detecting the transcription of Actin and $R b f$ in S2 cells and $0-12$ h-old embryos. RNA was isolated, reverse transcribed and analyzed as described (2.2.1.2.3).

To assess whether MDU is recruited to the transcriptionally repressed Rbf locus and repression of $R b f$ coincides with methylation of $\mathrm{H} 3 \mathrm{~K} 9$ and DNA, I performed XChIP assays. Chromatin was isolated from Drosophila embryos $(0-12 h)$ and $S 2$ cells and precipitated with antibodies to MDU, 5-methyl cytosine, and tri-methyl H3K9. PCR monitored the presence of $R b f$ enhancer in the precipitated DNA pools. MDU, 5-methyl cytosine, and tri-methyl $\mathrm{H} 3 \mathrm{~K} 9$ are present at the partially transcriptionally inactive $R b f$ locus in Drosophila embryos but not in S2 cells, which lack MDU (Figure 27).

Collectively, the results raised the possibility that silencing of the Rbf locus involves trimethylation of $\mathrm{H} 3 \mathrm{~K} 9$ by MDU and DNA methylation.

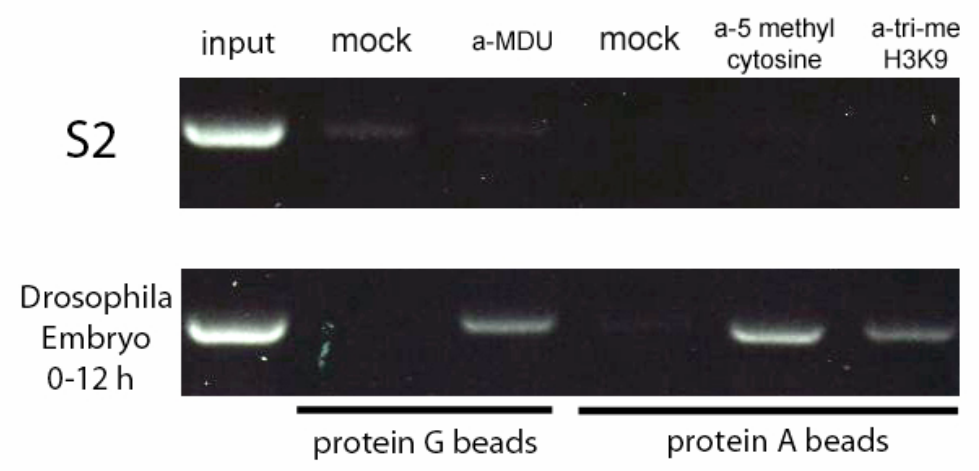

Figure 27. DNA and histone modification pattern of the Rbf locus in embryos and S2 cells. Ethidium bromide-stained agarose gels showing the reaction products of PCR assays detecting the presence of MDU, tri-methyl $\mathrm{H} 3 \mathrm{~K} 9$ and 5-methyl cytosine at the enhancer region of $R b f$ in $\mathrm{S} 2$ 
cells (top) and Drosophila embryos (bottom). Chromatin was isolated from S2 cells and 0-12hold embryos and precipitated with antibodies to MDU, tri-methyl H3K9 and 5-methyl cytosine or mock precipitated (mock). Chromatin was immunoprecipitated with use of protein-A or protein-G beads. PCR assays detected the enhancer in DNA isolated from $1 \%$ input material (input) and immunoprecipitated DNA pools.

\subsubsection{MDU-mediated transcription repression of $R b f$}

To investigate the role of MDU in silencing and DNA methylation at the Rbf locus, MDUmediated repression of $R b f$ was reconstituted in S2 cells. The cells were transfected with plasmids expressing GFP and MDU, MDU(H775L), or $\operatorname{MDU}(\mathrm{R} 436 \mathrm{C})$, which fails to bind methylated CpA motifs in vitro (3.3) Two days after transfection, cells were harvested and FACS isolated GFP positive cells (Figure 28).
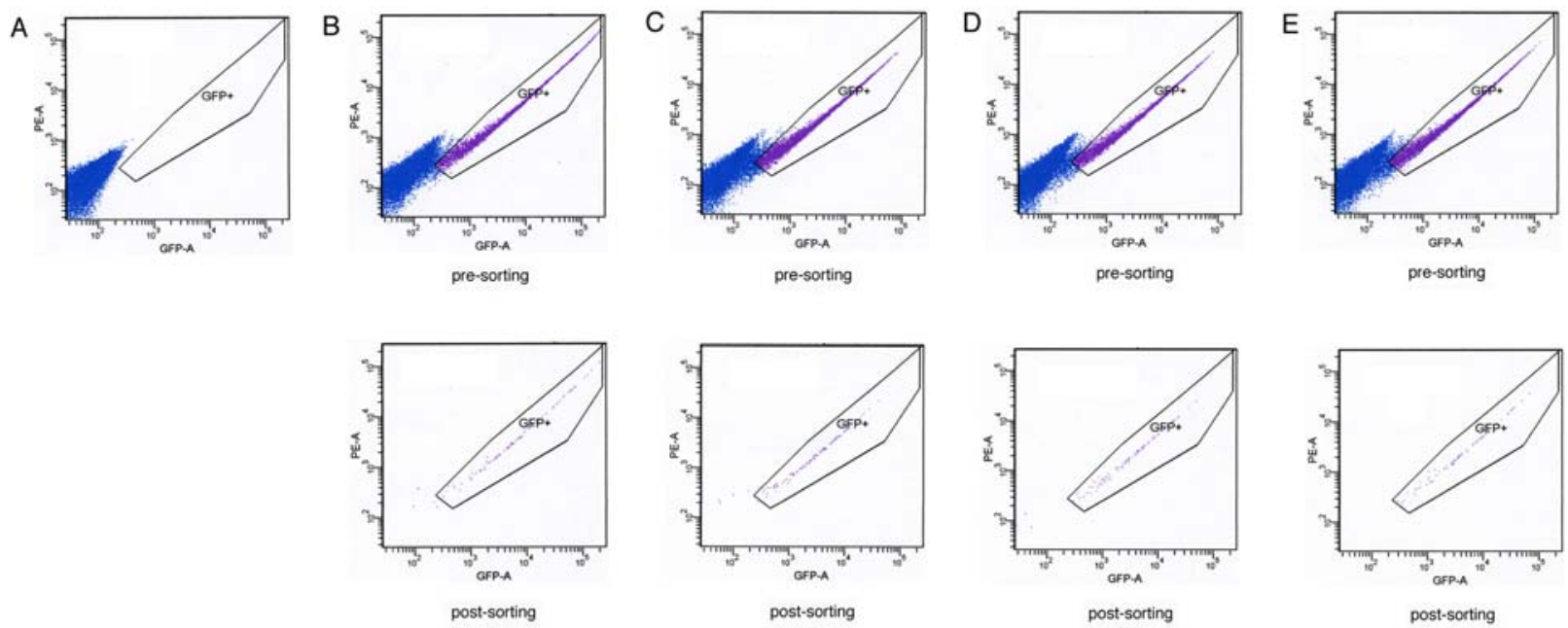

Figure 28. Schematic representation of FACS analysis. FACS analysis of (A) S2 cells, (B) S2 cells transiently expressing GFP and S2 cells transiently expressing GFP and (C) MDU, (D) $\mathrm{MDU}(\mathrm{H} 775 \mathrm{~L})$, or (E) MDU(R436C). The relative number of GFP-positive and -negative cells before and after sorting is shown for each group of cells.

RT-PCR monitored the transcription of Rbf, Mdu, dDnmt2 and Actin5C in GFPexpressing cells (Figure 29). Actin5C transcription was used to standardize the amount of RNA present in different RNA pools. $d D n m t 2$ is constitutively transcribed in all cell pools, which 
indicates that MDU is not involved in regulation of $d D n m t 2$ expression (Figure 29). This result supports our observation that $\mathrm{S} 2$ cells express $d D n m t 2$.

MDU transcription is detectable only in cells transfected with plasmids expressing wildtype or mutant MDU derivatives, which confirms that MDU is not transcribed in S2 cells and that MDU expression can be reconstituted in S2 cells by transient transfection assays (Figure 29).

Rbf was transcribed in S2 cells and GFP-expressing S2 cells, both of which lack MDU. In contrast, Rbf transcription was significantly reduced in cells expressing MDU, which indicates that MDU is involved in silencing of Rbf. In contrast, Rbf transcription was not significantly reduced in cells expressing $\mathrm{MDU}(\mathrm{H} 775 \mathrm{~L})$ or $\mathrm{MDU}(\mathrm{R} 468 \mathrm{C})$, suggesting that MDU-mediated repression of $R b f$ involves the activities of the SET and MBD domains.

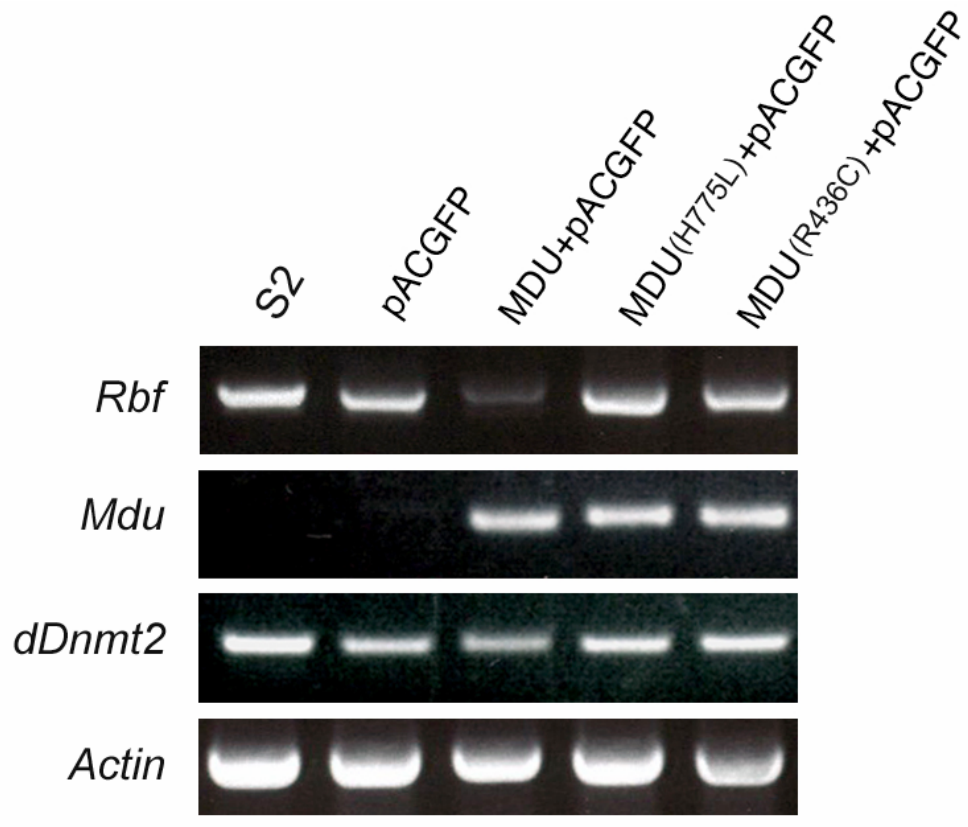

Figure 29. The activities of the SET and MBD domain of MDU are involved in silencing of $\boldsymbol{R} \boldsymbol{b f}$ transcription. Ethidium bromide-stained agarose gels showing the reaction products of PCR assays detecting the transcription of Rbf, Mdu, dDnmt2 and Actin in S2 cells, S2 cells transiently expressing GFP and S2 cells transiently expressing GFP and MDU, MDU(H775L), or MDU(R436C). RNA was isolated, reverse transcribed and analyzed as described (2.2.1.2.3). 


\subsubsection{MDU-mediated tri-methylation of H3K9 initiates de novo DNA methylation and silenicng of $R b f$}

To assess the role of the SET and MBD domains of MDU in silencing of Rbf expression, XChIP assays were performed. In vivo cross-linked chromatin was isolated from S2 cells expressing GFP and wild-type or mutant MDU-derivatives (see 2.2.10). Chromatin was sheared and immunoprecipitated with antibodies to MDU, 5-methyl cytosine, dDNMT2, and tri-methyl H3K9. DNA from precipitated chromatin was purified and used as a template in PCR assays that monitored the presence of the enhancer or exon 1 region of Rbf in DNA pools. Both regions are methylated in Drosophila eye imaginal discs in response to a hyperactive Notch signal trasnduction pathway (Ferres-Marco et al., 2006). Results of the XChIP assay are shown in Figure 30.
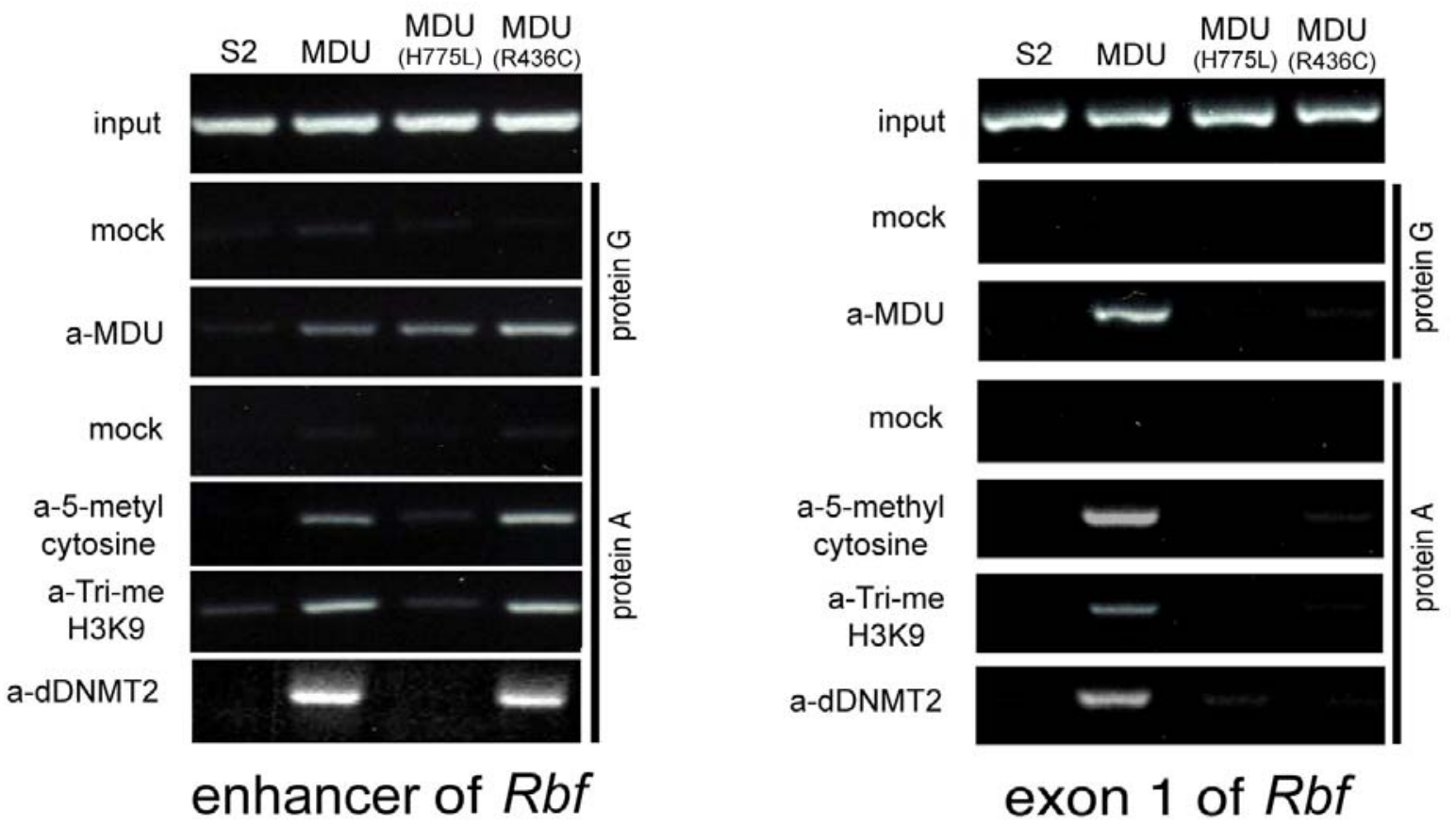

Figure 30. Tri-methylation of H3K9 by MDU induces de novo DNA methylation and silencing of $\boldsymbol{R} \boldsymbol{b f}$. Ethidium bromide-stained agarose gels showing the reaction products of PCR assays detecting the presence of MDU, dDNMT2, tri-methylated H3K9 and 5-methyl cytosine at the enhancer (left) and exon 1 region (right) of Rbf in chromatin isolated from S2 cells and S2 cells expressing MDU, MDU(H775L), or $\mathrm{MDU}(\mathrm{R} 436 \mathrm{C})$. Chromatin was precipitated with 
antibodies to MDU, tri-methyl H3K9, dDNMT2, and 5-methyl cytosine or mock precipitated (mock). Chromatin was immunoprecipitated with protein-A or protein-G beads. PCR assays detected the enhancer and exon 1 region of $R b f$ in DNA isolated from $1 \%$ input material (input) and immunoprecipitated DNA pools.

In S2 cells and GFP-positive S2 cells, MDU, dDNMT2, 5-methyl cytosine or tri-methyl $\mathrm{H} 3 \mathrm{~K} 9$ were not detected at the enhancer and exon 1 regions of $R b f$. In contrast, in cells expressing MDU, MDU, dDNMT2 and methylated H3K9 and DNA were present at the enhancer and exon 1 regions. Thus, repression of Rbf coincides with recruitment of MDU, MDU-mediated tri-methylation of $\mathrm{H} 3 \mathrm{~K} 9$, and DNA methylation.

In cells expressing MDU(H775L), MDU was detectable at the enhancer but not the exon 1 region. dDNMT2 and methylated DNA and H3K9 were not detected the enhancer and exon 1 region. Thus, MDU-mediated methylation of $\mathrm{H} 3 \mathrm{~K} 9$ is involved in repression of $R b f$. Attenuation of recruitment of dDNMT2 and DNA methylation indicates that MDU-mediated tri-methylation of $\mathrm{H} 3 \mathrm{~K} 9$ is involved in recruitment of dDNMT2 and subsequent de novo DNA methylation at the Rbf locus. In addition, the presence of MDU at the enhancer but not the exon 1 region suggests that MDU-mediated tri-methylation of $\mathrm{H} 3 \mathrm{~K} 9$ contributes to spreading of MDU from the enhancer to the exon 1 region.

In cells expressing MDU(R486C), MDU, tri-methyl H3K9, 5-methyl Cytosine, and dDNMT2 occupied the enhancer but not the exon 1 region, which suggests that the activity of the MBD is involved in spreading of MDU, dDNMT2 and the corresponding methylation of H3K9 and DNA from the enhancer to the exon 1 region.

In summary, the results reveal that tri-methylation of H3K9 by MDU plays a key role in silencing of $R b f$ by inducing the recruitment of dDNMT2, which results in DNA methylation and silencing. The MBD of MDU plays a key role in spreading MDU and dDNMT2 and the corresponding methylation of $\mathrm{H} 3 \mathrm{~K} 9$ and DNA on the Rbf locus. 
To confirm that MDU initiates DNA methylation at the Rbf locus, I performed methylationsensitive enzyme analysis and bisulfite sequencing. Both methods uncover the methylation status of DNA in a more accurate fashion than XChIP assays (Fraga and Esteller 2002). In addition, bisulfite sequencing can identify the position of 5-methyl cytosine within a gene (Fraga and Esteller 2002).

For the methylation-sensitive enzyme analysis, genomic DNA was isolated from S2 cells expressing GFP and wild-type or mutant MDU derivatives (2.2.1.17). The methylation-sensitive enzyme analysis was performed as described in 2.2.1.18 using the Hpall and Mspl endonucleases. Hpall cuts non-methylated but not methylated DNA, whereas the activity of Mspl is not sensitive to DNA methylation. Enzymatic digestion of the methylated Rbf locus by Hpall or Mspl prevents the detection of the enhancer and exon 1 region by PCR (2.2.1.18).

PCR assays monitored the presence of the enhancer and exon 1 of $R b f$ or the Psq gene as a control in nuclease- and mock-treated DNA pools (Figure 31).

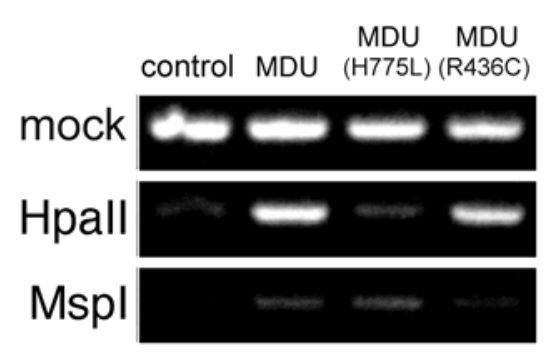

enhancer of $R b f$

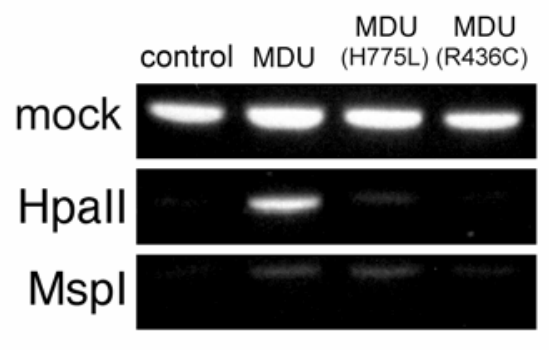

exon 1 of $R b f$

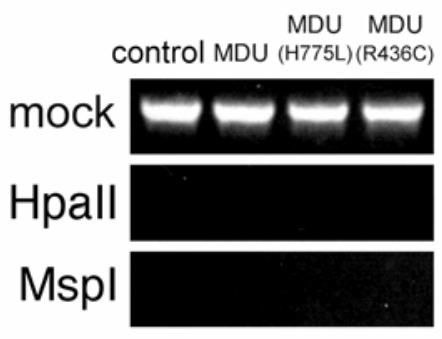

Psq

Figure 31. MDU-mediated tri-methylation of H3K9 induces de novo DNA methylation of Rbf. Ethidium bromide-stained agarose gels showing the reaction products of PCR assays detecting the enhancer (left) and exon 1 region (middle) of Rbf and pipsqueak (Psq, CG2368) in genomic DNA isolated from S2 cells and S2 cells expressing MDU, MDU(H775L), or MDU(R436C). Genomic DNA was mock treated (mock) or digested with Hpall, or Mspl. Both enzymes recognize the same DNA target sequence. Hpall is a methylation-sensitive restriction enzyme, whose activity is inhibited by methylation of the CpG motif in the target DNA sequence. The activity of Mspl is not sensitive to methylation. The PCR primer pairs used in this assay amplified DNA fragments containing multiple Hpall and Mspl site.

In GFP-positive S2 cells, the Rbf locus is not methylated, which results in enzymemediated degradation of the enhancer and exon 1 region by both Hpall and Mspl (Figure 31). The enhancer and exon 1 regions are detectable in the Hpall-treated genomic DNA isolated 
from cells expressing MDU, which confirms that the Rbf locus is methylated in the presence of MDU (Figure 31).

In the DNA from cells expressing MDU(H77L), the enhancer and exon regions were not detected, which confirms that the Rbf locus is not methylated in the absence of MDU-mediated tri-methylation of H3K9 (Figure 31).

In DNA isolated from cells expressing MDU(R486C), the enhancer but not exon 1 region was detected, which confirms DNA methylation at the enhancer region and loss of spreading of DNA methylation through attenuation of the MBD-activity of MDU (Figure 31).

In all cases, treatment of the DNA with non-methylation-sensitive enzyme Mspl degraded the enhancer and exon 1 regions of $R b f$, which indicates that the employed assay specifically detects methylated DNA.

The results of the methylation-sensitive enzyme analysis were confirmed by bisulfite sequencing assays (Figure 32, Figure 33).

First, genomic DNA was isolated from $S 2$ cells and $S 2$ cells expressing wild type or mutant MDU derivatives. The DNA was subjected to bisulfite sequencing assay (2.2.1.19). Three independent assays were performed. The partial results of one assay are shown in Figure 32. 

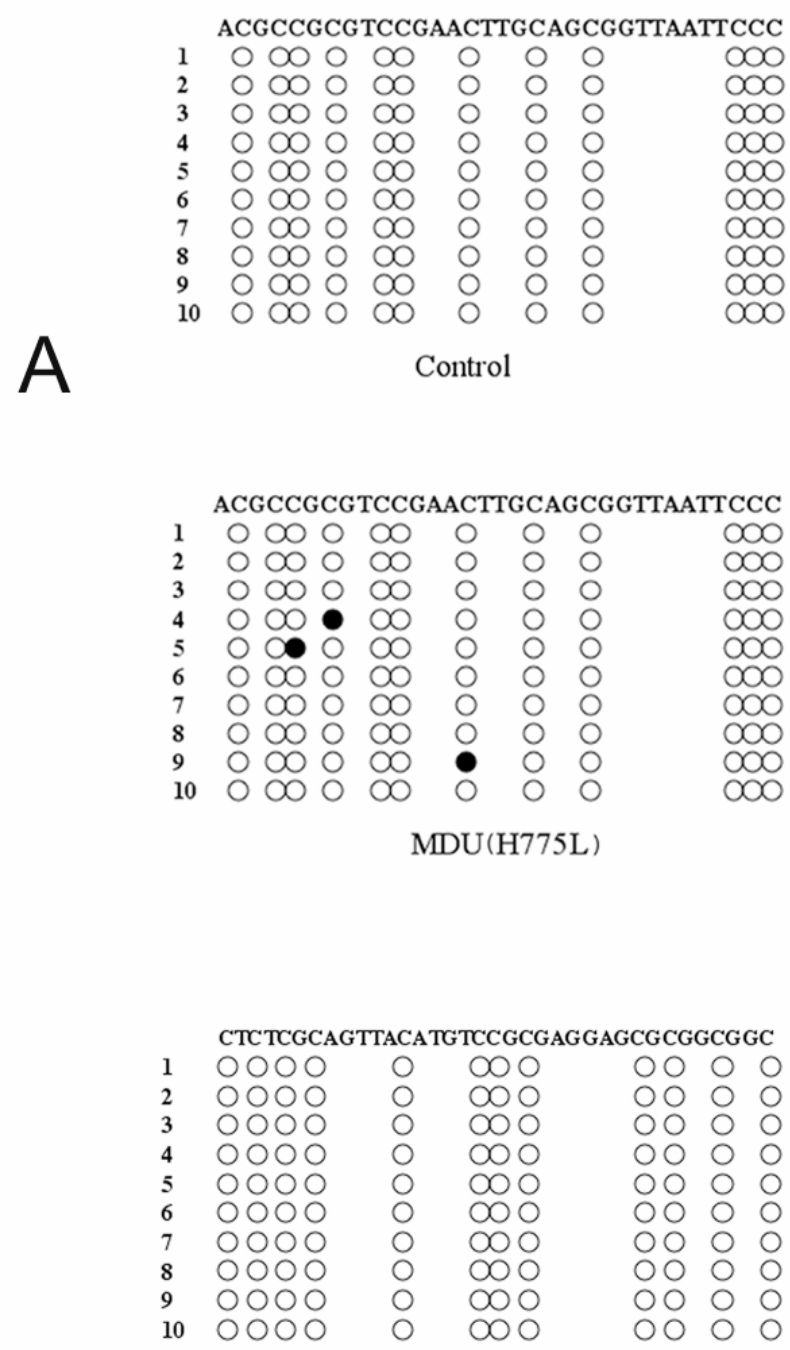

B

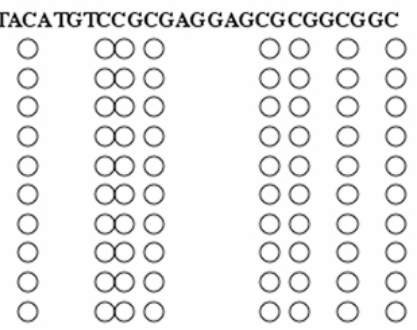

Control

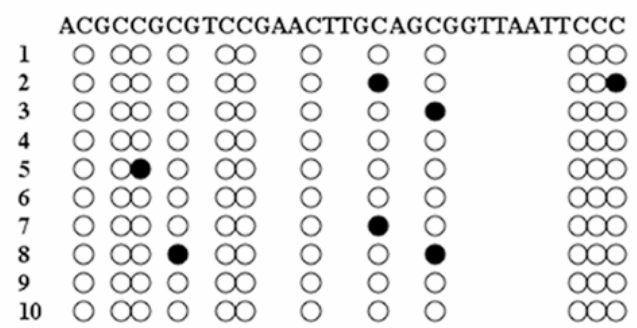

Wild type MDU

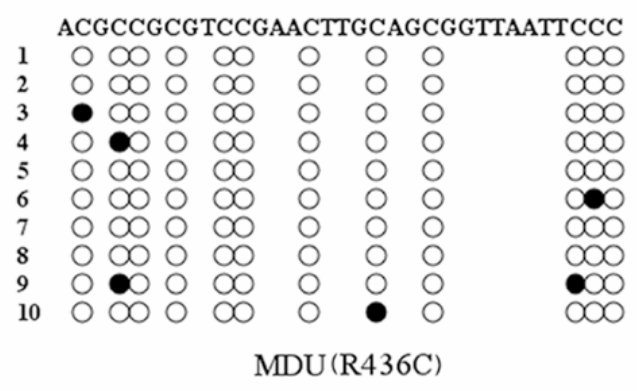

Figure 32. Schematic representation of bisulfite sequencing assays. Genomic DNA was isolated from S2 cells and S2 cells expressing MDU, MDU(H775L), or MDU(R436C). Bisulfite sequencing was performed as described (2.2.1.19). The enhancer (A) and exon 1 region (B) of $R b f$ were amplified by PCR from bisulfite-treated DNA, cloned, and sequenced. 10 clones were 
sequenced for each test group. The schematic drawings indicate DNA methylation within a 33bp fragment of the enhancer and a 36-bp fragment of the exon 1 region. Unmethylated cytosines are represented by a blank circle; methylated cytosines are represented by filled circles. The sequence of the enhancer fragment is ACGCCGCGTCCGAACTTGCAGCGGTTAATTCCC. The sequence of the exon 1 fragment is CTCTCGCAGTTACATGTCCGCGAGGAGCGCGGCGGC.

The overall methylation level of every group in each independent assay was calculated as the percentage of methylated cytosines to total number of cytosines present in the amplified DNA fragments. The results of all 3 independent assays are shown in Figure 33.
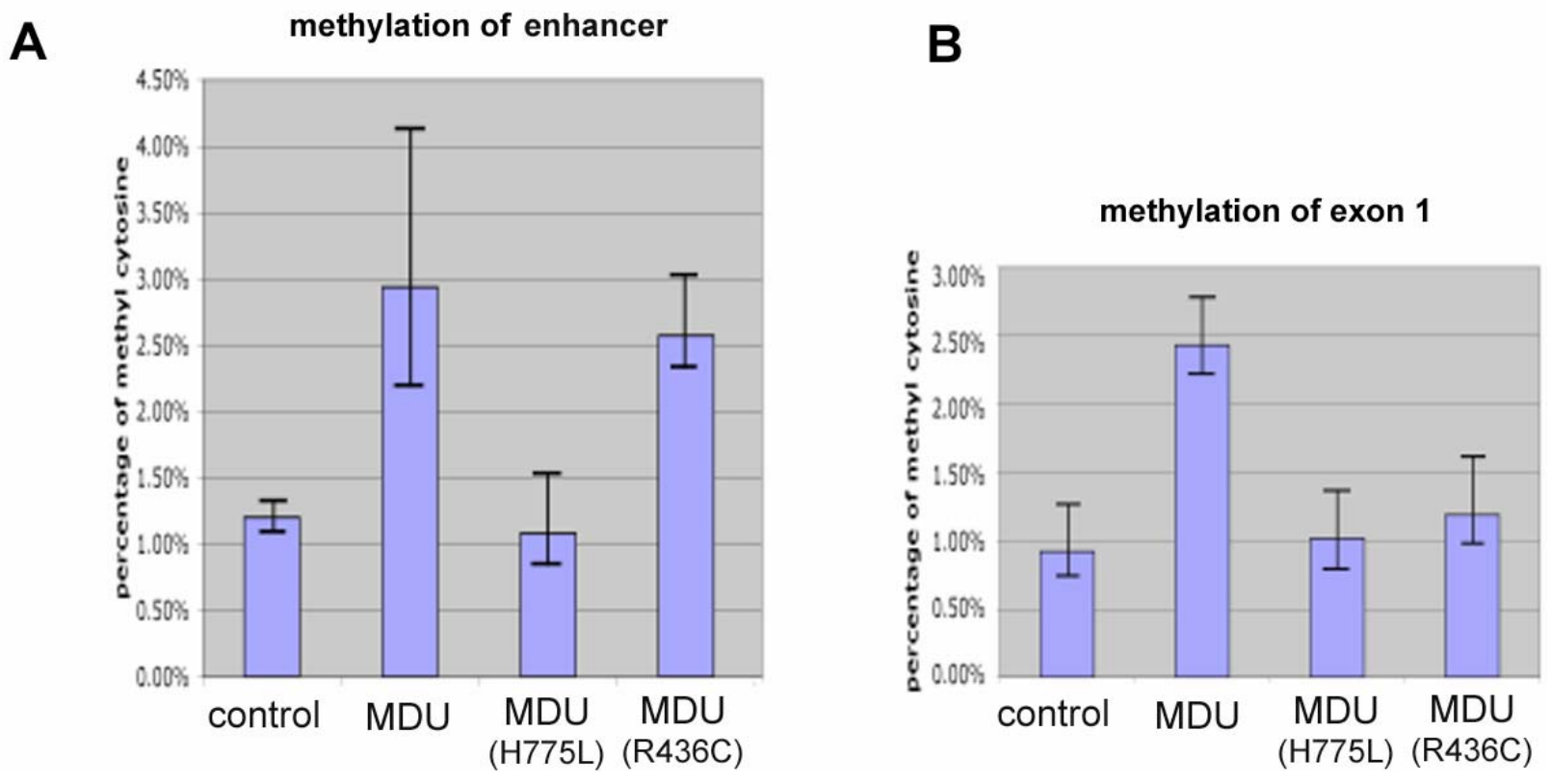

Figure 33. DNA methylation at the enhancer and exon 1 regions of $R b f$. Schematic representation of the results of bisulfite sequencing assays described in 2.2.1.19. For each test group 10-13 clones were sequenced. The percentage of methylated cytosines detected in the enhancer $(A)$ and exon I regions $(B)$ is indicated

In S2 cells or S2 expressing MDU(H77L), 1.2\% of all cytosines were methylated in the enhancer region. Methylation at the enhancer increases to $2.2 \%-4.1 \%$ in cells expressing MDU and $2.3 \%-3 \%$ in cells expressing MDU(R436C). 
We detected $1.2 \%$ methylation at the exon 1 region in S2 cells and S2 cells expressing $\mathrm{MDU}(\mathrm{H} 775 \mathrm{~L})$ or $\mathrm{MDU}(\mathrm{R} 436 \mathrm{C})$. In contrast, methylation of the exon 1 region was significantly enhanced in cells expressing MDU (2.2\%-2.7\%).

Collectively, the results confirm that MDU initiates de novo DNA methylation at the enhancer and exon 1 regions of Rbf and confirm the role of the SET and MBD domains in initiation and spreading, respectively, of DNA methylation. 


\subsection{Knocking down Mdu in the eye disc}

RBF is involved in eye development in Drosophila by controlling the correct timing and maintenance of G1 cell cycle arrest in eye imaginal discs (Du et al., 1996a; Du et al., 1996b; Du and Dyson 1999).

RBF is negatively regulated by phosphorylation through cyclin D or cyclin $E$. Expression of RBF-280, a mutant form of RBF with four putative Cdk phosphorylation sites mutated that can no longer be regulated by cyclin $D$ or cyclin $E$, results in the development of a very rough eye with fused ommatidia and almost complete loss of bristles (Xin et al., 2002).

A recent study suggests that $R b f$ activity is regulated at the transcriptional level (FerresMarco et al., 2006). To investigate the role of MDU in regulation of Rbf expression in Drosophila eye imaginal discs, I used RNA interference (RNAi) to attenuate the expression of $M d u$ in developing eye imaginal discs. RNAi was induced by use of the GAL4/UAS system. The Gal4/UAS system is based on transcriptional activation of a GAL4-dependent reporter gene by the yeast activator Gal4 (Brand and Perrimon 1993). I used the reporter strain 12196-48 [a gift from Professor Andrew Lambertsson (University of Oslo, Norway)]. 12196-48 carries a Gal4dependent RNAi reporter construct, which transcribes 540 dsRNA targeting $M d u$ and is under the control of DNA target sites (UAS) for Gal4.

The reporter strain was crossed with the driver strain 6313 (Bloomington Stock center). 6313 expresses Gal4 under the control of the lozenge (Iz) enhancer in eye imaginal discs (Nichols et al., 1996). The adult flies of the genotype (6313/12196-48 flies) were collected, and the eye phenotype of $6313 / 12196-48,6313$, and $12196-48$ flies was visualized by scanning electron microscope (SEM).

Compared to the wild type, 12196-48 and 6313 flies showed a normal eye phenotype. In contrast, an aberrant eye phenotype was observed in 6313/12196-48 flies. The eyes of 6313/12196-48 flies contain deformed and fused ommatidia and lack eye bristles almost completely. The observed phenotype resembles the rough eye phenotype observed in flies expressing constitutively active RBF (Xin et al., 2002). The results support the hypothesis that Mdu downregulates Rbf transcription and that $M d u$ is involved in eye development. 

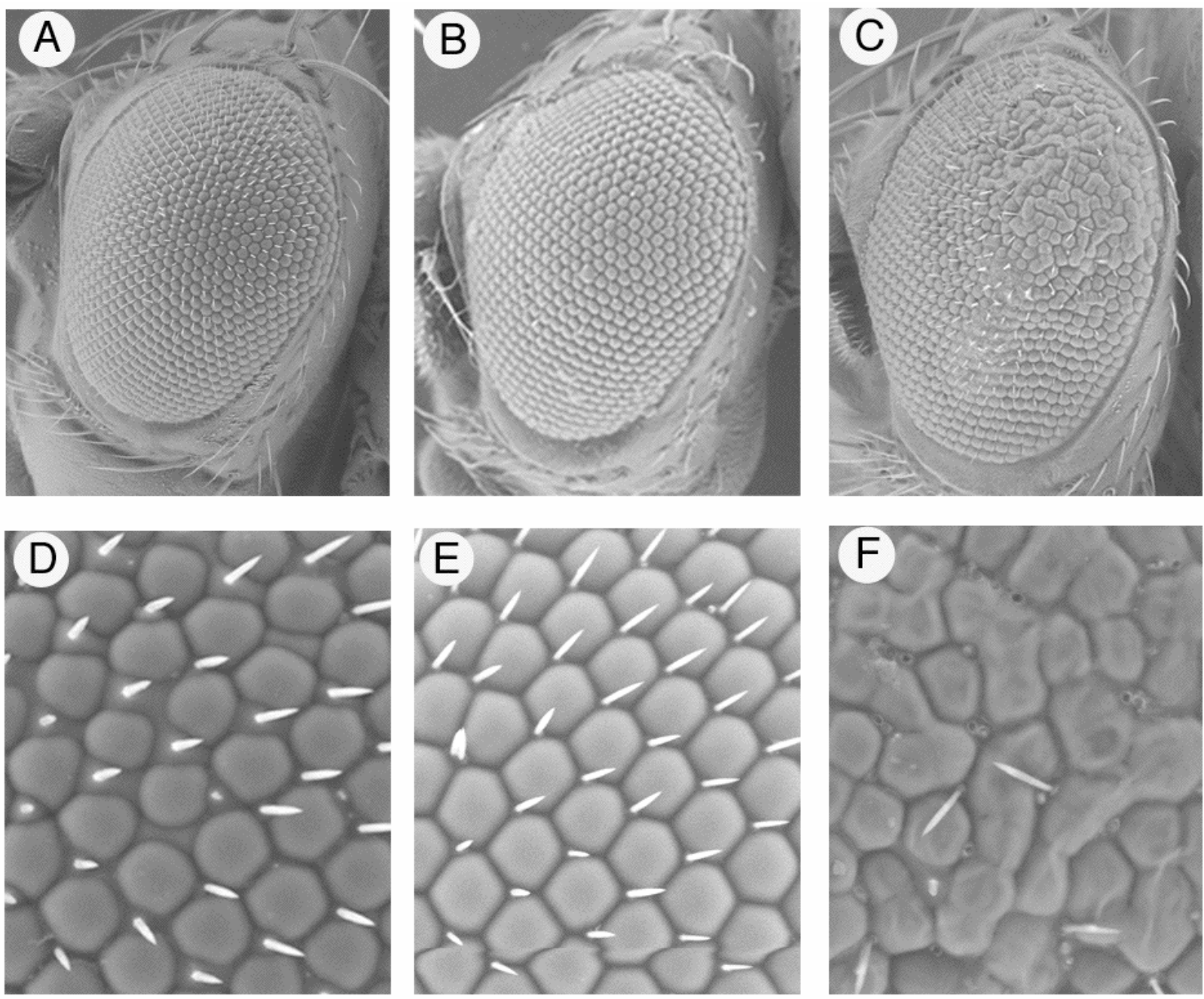

Figure 34. Mdu is involved in eye development. Scanning electron microscope (SEM) images of eye phenotype of eyes of $(\mathbf{A})$ the Gal4 driver 6313, (B) the reporter 12196-48, and (C) $6313 /+; 12196-48 /+$. The magnification is 50 fold. (D-F). Magnified (1000x) images of the areas delineated by a white box in (A-C). Note the fusion of ommatidia and the absence of bristles in eyes of $6313 /+; 12196-48 /+$ flies. 


\section{Discussion}

Methylated DNA and histone $\mathrm{H} 3 \mathrm{~K} 9$ are two major epigenetic signals associated with gene silencing and heterochromatin formation. The mechanisms resulting in methylation of DNA and H3K9 are interdependently connected (Tamaru and Selker 2001; Fuks 2003b; Strunnikova et al., 2005).

Drosophila contains DNA and histone methylation systems (Lyko et al., 2000a; Beisel et al., 2002; Schotta et al., 2002; Marhold et al., 2004). The role of histone methylation in the structure and function of Drosophila chromatin has been thoroughly investigated (Ebert et al., 2006). In contrast, the role of DNA methylation in Drosophila and the enzymes and mechanisms involved in de novo DNA methylation are only now being discovered.

The results of this work reveal that the Drosophila MBD/SET domain protein MDU plays a key role in de novo DNA methylation and gene silencing in Drosophila. MDU is a multifunctional protein: the MBD of MDU binds methylated CpA-motif, whereas the SET domain tri-methylates H3K9. MDU-mediated tri-methylation of H3K9 initiates recruitment of the DNA methyltransferase dDNMT2 to the Rbf locus, resulting in de novo DNA methylation of the Rbf locus and culminating in silencing of $R b f$ transcription. The obtained results support a model in which MDU-mediated tri-methylation of H3K9 facilitates initiation and self-reinforced propagation of de novo DNA methylation in Drosophila.

The results uncover a role for MDU in DNA methylation and gene silencing in Drosophila and imply that members of the phylogenetically conserved family of SET/MBD proteins play a key role in gene-specific de novo DNA methylation in animals and plants.

\subsection{Functional MDU is encoded by CG30426}

Mdu was initially identified as a 3,948-bp open reading frame (CG12196) in the Drosophila genome (Stabell et al., 2006). More recent annotations separated the Mdu locus into two different genes, CG30422 and CG30426. CG30426 consists of 2716 bp open reading frame separated into six exons and encodes for the MBD and SET domains of MDU. CG30422 consists of only one 1,049-bp exon. 
Studies by others and our laboratory have demonstrated that the Mdu locus produces only a 3,948 bp transcript that corresponds to the predicted transcript of CG12196 (MoraBermúdez 2002; Tzeng et al., 2007, Seum et al., 2007 and Clough et al., 2007).

The transcription of Mdu starts in the early embryo and persists in the larval and adult stages of Drosophila (Stabell et al., 2006; Tzeng et al., 2007, Seum et al., 2007; Yoon et al., 2008;). Several groups have analyzed the transcription pattern of $M d u$ and reported contradictory results: Stabell et al. stated that $M d u$ is not transcribed in $0-3 \mathrm{~h}$-old embryos. Transcription of Mdu starts in 4h-old embryos and persists in larvae, pupae and adult flies (Stabell et al., 2006). In contrast, Yoon et al. detected Mdu transcription in 0-4h embryo but not in pupae (Yoon et al., 2008). The authors observed an oscillation of $M d u$ transcription during the life cycle of Drosophila. Expression of Mdu was detected in 0-4h embryo and first- and third-instar larvae but was severely reduced after each of the 3 stages (Yoon et al., 2008).

The results of this study suggest that functional MDU protein corresponds to the CG30426 locus rather than the CG12196 locus. In immunoprecipitation assays with monoclonal anti-MDU antibody we did not detect a protein corresponding to full-length CG12196 (141.97KDa). Instead mass-spectrometry analysis detected two derivatives of 63-82 kDa (Figure 11), which correspond to the CG30426 locus.

In Western blot assays, Clough et al. detected two MDU proteins (170 kDa and $140 \mathrm{kDa}$ ) in Drosophila ovaries (Clough et al., 2007). A second study showed MDU expressed as a 142$\mathrm{kDa}$ protein in adult flies (Stabell et al., 2006). However, the identity of the detected immunoreactive proteins was not confirmed by mass-spectrometry in either study. The antibodies used in both studies recognize the $\mathrm{NH}_{2}$-terminus of the protein encoded by CG12196 but not the protein encoded by CG30426. The detection of MDU proteins of different sizes in extracts from ovaries, adults and embryos suggests that different MDU derivatives are expressed during different developmental stages of Drosophila. The Mdu locus is transcribed as a 4000-nt mRNA throughout development, which suggests that the different MDU isoforms are not a result of alternative splicing. Alternatively, the expression of MDU proteins could be subject to delicate translational or post-translational regulation, such as protease-mediated protein cleavage, which results in different isoforms of MDU. Protease-mediated cleavage has been demonstrated for epigenetic regulators such as mammalian MLL and Drosophila Trithorax. In both cases, protease-mediated cleavage results in the production of active proteins (Kuzin et al., 1994; Hsieh et al., 2003). The differential regulation of Mdu expression at the transcriptional and translational levels may allow MDU to exert different biological functions during different stages of Drosophila development. 


\subsection{MDU is a multifunctional transcriptional regulator}

\subsubsection{The MBD of MDU binds methylated CpA motifs}

MBD proteins bind methylated DNA and play important roles in epigenetic silencing ( $\mathrm{Ng}$ et al., 2000; Bienvenu and Chelly, 2006). Our experiments revealed that the MBD of MDU is a functional methylated DNA binding domain and binds methylated $\mathrm{CpA}$ motifs. Unlike mammalian MeCP2, the MBD of MDU does not bind methylated CpG-motifs, which suggests that MBD proteins in invertebrates contact methylated DNA motifs other than CpG-motifs. That hypothesis is supported by recent studies suggesting that the MBD does not exclusively bind methylated CpG motifs but rather is a functional module, whose binding affinities to methylated DNA have adopted in response to the various DNA methylation patterns present in various species (Pitto et al., 2000; Scebba et al., 2003; Marhold et al., 2004). Vertebrates methylate their genomes mainly at symmetrical $\mathrm{CpG}$ sequences, and human MBD proteins such as MeCP2 and MBD1 showed a preference for CpG-methylated DNA. In carrot, two classes of MBD proteins have been identified. The first shows high affinity for sequences containing 5-methyl cytosine in a canonical $\mathrm{CpG}$ methylation context, whereas the second efficiently binds 5-methyl cytosine within both CpXpX and CpXpG (X=A, T, or C) tri-nucleotides (Pitto et al., 2000). The Arabidopsis MBD protein AtMBD5 can bind both symmetrically methylated $\mathrm{CpG}$ and asymmetrically methylated CpXpX sequences (Scebba et al., 2003). The Drosophila genome is predominantly methylated at asymmetrical CpA and CpT sequences (Lyko et al., 2000a; Gowher et al., 2003). Similar to MDU, Drosophila dMBD2/3, the ortholog of vertebrate MBD2 and MBD3, binds methylated CpT/A-motifs (Marhold et al., 2004). Thus, MBD proteins in Drosophila may preferentially bind methylated $\mathrm{CpA}$ and/or $\mathrm{CpT}$-motifs, which indicates that the binding specificity of fly MBD proteins corresponds well with the DNA methylation pattern of Drosophila.

How does MDU bind methylated CpA motifs? Why does MDU bind methylated CpA and not other methylated motifs?

The comparison of the primary sequence of the MBD of MDU and other proteins, especially the MBD of MeCP2, reveals that key amino acids involved in the binding of MeCP2 to methylated CpG-motifs such as R106 and R133 are conserved in the MBD of MDU. Only the position of amino acid residue corresponding to R111 of MeCP2 is not conserved but was replaced with a glutamine-residue $(\mathrm{Q})$ in the MBD of MDU. Several MBD proteins (e.g., MBD4, ARBP) contain a QR111 motif, whereas MDU contains a RQ motif. The association of the MBD 
of MDU with methylated CpA motifs indicates that the reversal of the QR111 motif to RQ does not affect the ability of the MBD to bind methylated DNA. However, the switch of the RQ motif in the MBD of MDU may allow MDU to bind methylated CpA instead of CpG motifs.

Another conserved amino acid in the MBD of MDU is R436, which corresponds to R133 in MeCP2. In our study, the mutation R436C greatly attenuated the binding of the MBD of MDU to methylated DNA (3.3), which suggests that R436, like R133 of MeCP2, plays a key role in the association of MDU with methylated DNA.

The structures of three different MBD motifs (human MeCP2 and MBD1, chicken MeCp2) have been solved (Ohki et al., 1999; Wakefield et al., 1999; Heitmann et al., 2003. Figure 35. Structure of human MeCP2's MBD).
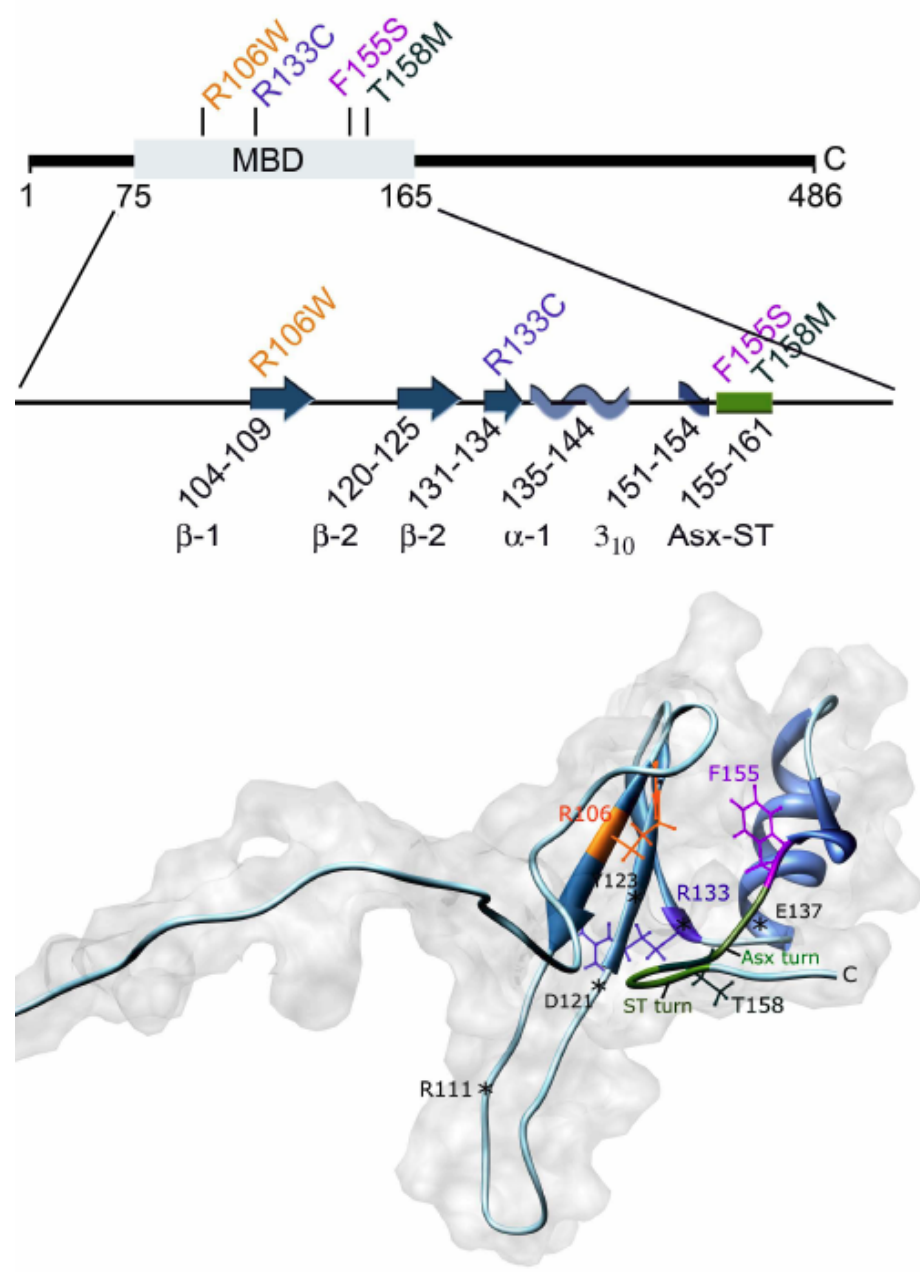

Figure 35. Structure and conserved residues in the MBD domain of human MeP2. (Top) Schematic drawing of MeP2 indicating the location of the MBD and the secondary structures of the MBD. (Bottom) NMR-based model of the structure of the MBD with DNAbinding residues marked with asterisks (Ghosh et al., 2008). 
The MBD forms a wedge-shaped structure composed of a $\beta$-sheet superimposed over a $\alpha$-helix and loop. Amino acid side chains in two of the $\beta$-strands along with residues immediately $\mathrm{NH}_{2}$ terminal to the $\alpha$-helix interact with the cytosine methyl groups within the major groove, providing the structural basis for selective recognition of methylated CpG dinucleotides (Ohki et al., 2001; Wade and Wolffe 2001) (Figure 35).

Amino acid residues R106, R133, F155 and T158 in the MBD domain of MeCP2 are involved in the binding of MBD to methylated DNA. Mutations in those amino acid residues have been associated with Rett syndrome, a severe X-linked neurodevelopmental disorder in humans (Amir et al., 1999). Another important residue for methyl DNA binding activity of MeCP2 is R111. Mutations in R111G, R106W and R133C greatly decrease the binding of MeCP2's MBD to methyl DNA (Free et al., 2001)(Figure 36).

\begin{tabular}{|c|c|c|c|c|c|}
\hline Residue $^{A}$ & Structural location ${ }^{\underline{2}}$ & Conserved $^{e}$ & Chemical shift change $e^{d}$ & $\mathrm{nM}$ protein & DNA binding \\
\hline & & & & & \% bound ${ }^{*}$ \\
\hline \multirow[t]{3}{*}{ wt } & & & & 20 & 61 \\
\hline & & & & 200 & 97 \\
\hline & & & & 2000 & 99 \\
\hline \multirow[t]{3}{*}{$\mathrm{R} 106(\mathrm{~W})^{*}$} & Core & Yes & - & 20 & 11 \\
\hline & & & & 200 & 21 \\
\hline & & & & 2000 & 31 \\
\hline \multirow[t]{3}{*}{$\mathrm{R} 111(\mathrm{G})$} & B-C loop & Yes & ++ & 20 & 0 \\
\hline & & & & 200 & 0 \\
\hline & & & & 2000 & 3 \\
\hline \multirow[t]{3}{*}{ Y123(A) } & Strand C & Yes & + & 20 & 19 \\
\hline & & & & 200 & 52 \\
\hline & & & & 2000 & 61 \\
\hline \multirow[t]{3}{*}{ Y123(D) } & Strand C & Yes & + & 20 & 14 \\
\hline & & & & 200 & 28 \\
\hline & & & & 2000 & 38 \\
\hline \multirow[t]{3}{*}{$\mathrm{I} 125(\mathrm{~A})$} & Strand C & Yes & - & 20 & 11 \\
\hline & & & & 200 & 76 \\
\hline & & & & 2000 & 71 \\
\hline \multirow[t]{3}{*}{ R133(C)* } & Strand D & Yes & ++ & 20 & 7 \\
\hline & & & & 200 & 8 \\
\hline & & & & 2000 & 18 \\
\hline \multirow[t]{3}{*}{ F155(S)* } & Core & Yes & - & 20 & 50 \\
\hline & & & & 200 & 61 \\
\hline & & & & 2000 & 61 \\
\hline \multirow[t]{3}{*}{$\mathrm{T} 158(\mathrm{M})^{*}$} & C-terminus & Yes & - & 20 & 60 \\
\hline & & & & 200 & 70 \\
\hline & & & & 2000 & 71 \\
\hline
\end{tabular}

Figure 36. Amino acids involved in interaction of MBD of MeCP2 with methylated CpGmotifs. Mutated MBDs with single amino acid exchange mutations were assayed for binding to a methylated 27-bp duplex oligonucleotide containing a single, symmetrically methylated CpG dinucleotide by using EMSA. Each mutated MBD was assayed at three concentrations (20, 200 and $2000 \mathrm{nM}$ ). DNA binding activity was calculated as the percentage of DNA retained by mutated or wild type MBD to total DNA applied to the assay. Amino acids mutated are indicated by their single letter codes with numbering corresponding to their position in MeCP2. Substituted amino acids are indicated in parentheses, and residues mutated in cases of Rett syndrome are indicated by asterisks (Free et al., 2001). 
Among the three mutations R111G, R106W and R133C in the MeCP2 MBD domain, the $\mathrm{R} 111 \mathrm{G}$ resulted in the most severe impairment in methylated DNA binding activity. $\mathrm{R} 133 \mathrm{C}$ is the second strongest mutation (Free et al., 2001). Although the mutation R133C resulted in greatly decreased methyl DNA binding activity, the mutation is not believed to affect the structure of MBD domain (Free et al., 2001).

Another study demonstrated that all 4 mutations (R106W, R133C, F155S, T158M) located in the MBD domain of MeCP2 have profound and diverse effects on the structure, stability, and DNA-binding properties of the MBD (Ghosh et al., 2008). The mutations R133C, F155S, and T158M reduce the thermal stability of the MBD (Ghosh et al., 2008). Thermal stability of the wild-type protein is increased in the presence of unmethylated DNA, and further enhanced by DNA methylation. DNA-induced thermal stability was also observed for mutant proteins but to a lesser extent (Ghosh et al., 2008). According to this study, the mutant R133C causes structural changes in the MBD. Both the full-length mutant and the MBD mutant of R133C show reduced thermal stability as compared with the wild type, and the EMSA data show reduced binding to methylated DNA (Ghosh et al., 2008).

The crystal structure of the MBD of MeCP2 complexed to methylated DNA (Ho et al., 2008) revealed that contrary to the traditional model proposing that the binding specificity of the MBD depends on hydrophobic interactions between cytosine methyl groups and a hydrophobic patch within the MBD, the methyl groups predominantly contact hydrophilic surfaces that include tightly bound water molecules. The only amino acid residues of the MBD of MeCP, which directly interact with DNA, are D121, R111, and R133 (Ho et al., 2008). Of the 25 interactions occurring between the methyl cytosine groups of the DNA and the MBD, only two interactions between methyl cytosine and R133 are classically hydrophobic in character. Also, hydrogen bonds are formed between the symmetrical arginine fingers (R111 and R133) and each guanine of the methyl CpG pair (Ho et al., 2008). Thus, R133 in MeCP2 has unique and indispensable functions for the structure and function of the MBD.

Our results are consistent with other studies indicating that R436 of MDU (R133 of MeCP2) is important for the methyl DNA binding activity of the MBD. Further functional studies of other amino acid residues in the MBD of MDU and the study of the crystal structure of the MBD are necessary to demonstrate the mechanism of MBD of MDU recognizing and binding methylated CpA motifs in target DNA. 


\subsubsection{Product specificity of the HMT activity of MDU}

HMTs can mono-, di- and tri-methylate target lysine residues (Zhang et al., 2003b). The methylation status of lysine residues plays an important role in the histone code hypothesis, because the methylation status of the lysine residue determines the biological function of histone methylation (Ruthenburg et al., 2007). For example, tri-methylated H3K4 has been associated with transcriptional activation, whereas di-methylated H3K4 has been detected in eu- and heterochromatin (Santos-Rosa et al., 2002).

Previous studies revealed that the structure of the lysine access channel in the SET domain determines the product specificity of HMTs (Xiao et al., 2003b, Trievel et al., 2003, Zhang et al., 2003b) (Figure 37).
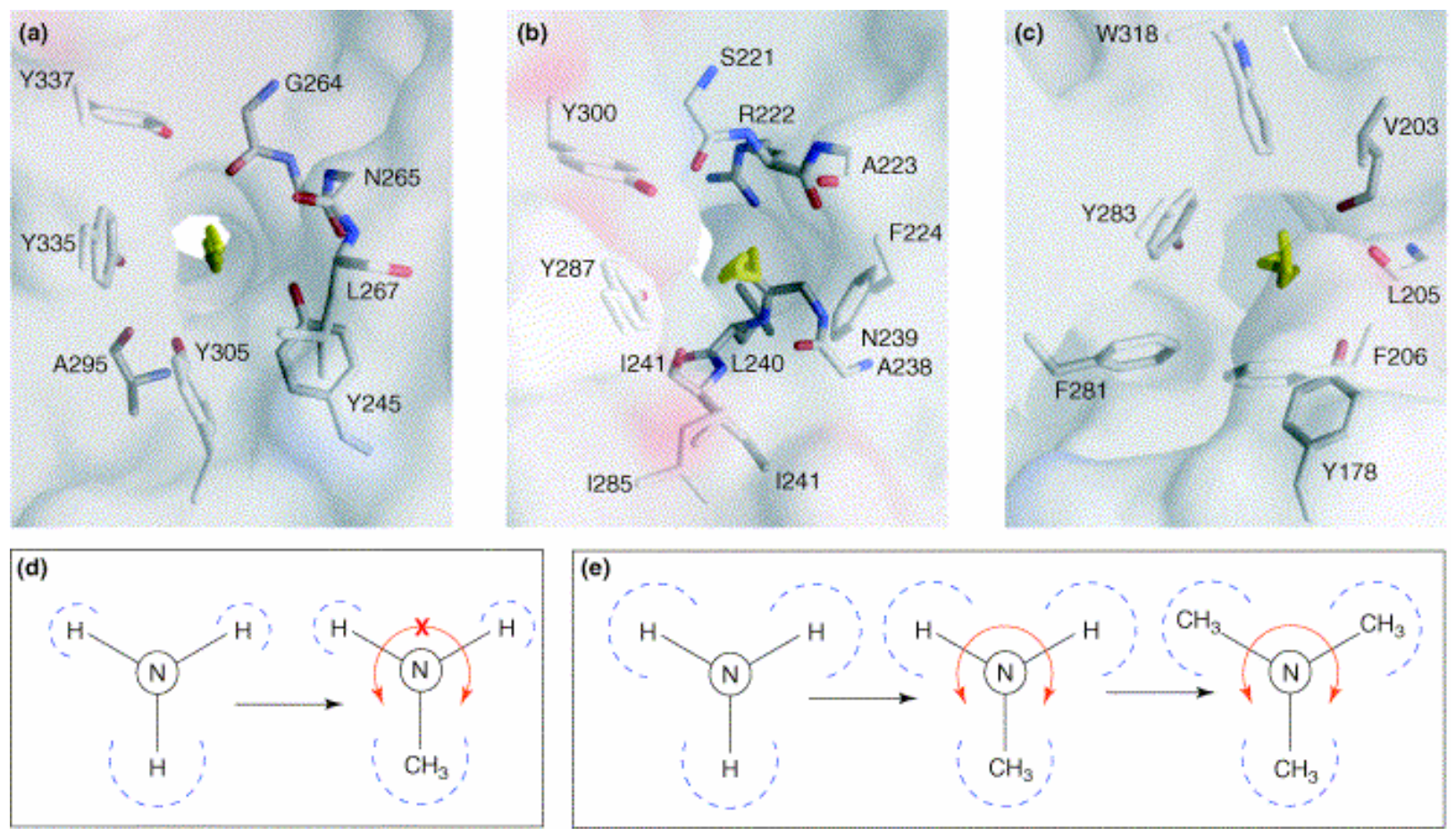

Figure 37. The geometry of the lysine access channel determines the number of methyl groups that can be transferred. (a-c) Surface representations of the lysine access channels of the three ternary structures viewed from the peptide-binding side: (a) Set7/9, (b) Rubisco LSMT and (c) DIM-5. Key residues are shown in stick formation, and the substrate lysine side chain is shown in yellow. (d,e) Diagrams illustrating how the channel geometry either prevents rotation around the C--N bond after the addition of a methyl group, (d) as in the case of SET7/9, or 
allows rotation and therefore the addition of further methyl groups, (e) as in the case of DIM-5 and LSMT (Xiao et al., 2003a).

The lysine access channel is a narrow channel that connects the cofactor-binding site on one surface with the substrate-binding site on the opposite surface of the domain (Xiao et al., 2003a). The geometry of the lysine access channel determines the number of methyl groups that can be transferred.

In Figure 37, the lysine access channels of the three HMTase (SET7/9, DIM-5 and Rubisco LSMT) ternary complex structures are shown from the viewpoint of the peptide-binding side. DIM-5 and Rubisco LSMT catalyze tri-methylation of their target lysine residues, whereas SET7/9 adds just a single methyl group to its lysine substrate (Xiao et al., 2003b; Trievel et al., 2003; Zhang et al., 2003b). The authors of these studies propose that the geometry and shape of the bottom of the access channel are responsible for determining how many methyl groups the SET domain can methylate. This endpoint is achieved either by preventing rotation around the $\mathrm{C}-\mathrm{N}$ bond of the lysine side chain after the addition of a methyl group or permitting the rotation (Figure 37 ).

Our results indicate that MDU predominantly tri-methylates $\mathrm{H} 3 \mathrm{~K} 9$ in vitro and mediates tri-methylation of $\mathrm{H} 3 \mathrm{~K} 9$ at target genes in vivo.

A recent study demonstrated that MDU mono-, di- and tri-methylates H3K9 in a sequential manner (Tzeng et al., 2007). In vitro HMTase assay was performed with immunopurified MDU from transfected S2 cells. At different times, the reaction products were analysed by Western blotting with antibodies against different forms of methylated H3K9. The results revealed that MDU catalyzed $\mathrm{H} 3$ methylation switches gradually from predominantly mono-methylation to tri-methylation (Tzeng et al., 2007). We cannot rule out that MDU methylates $\mathrm{H} 3 \mathrm{~K} 9$ in a sequential fashion. However the presence of only tri-methylated $\mathrm{H} 3 \mathrm{~K} 9$ at MDU target genes in vivo provides strong evidence that MDU establishes tri-methylated $\mathrm{H} 3 \mathrm{~K} 9$ at target genes rather than mono-, di- and tri-methylated H3K9. The HMTs DIM-5 and LSMT mediate tri-methylation of $\mathrm{H} 3 \mathrm{~K} 9$ in a sequential fashion. Thus the sequential methylation of $\mathrm{H} 3 \mathrm{~K} 9$ by MDU in vitro reflects on the mechanism by which MDU methylates H3K9 of HMTs rather than revealing the product specificity of the HMT-activity of MDU. Collectively, the results support a model in which MDU tri-methylates $\mathrm{H} 3 \mathrm{~K} 9$ in vitro.

This model is challenged and confirmed by contradicting in vivo studies of the HMTactivity of MDU. 
Tzeng et al. analyzed methylation of histone 3 in third-instar larvae of Drosophila by Western blotting (Tzeng et al., 2007). Mono-, di- and tri-methylated H3K9 were detected in wild type larvae, whereas mono-, di- and tri-methylation of H3K9 were decreased in MDU mutant larvae. All three methylated $\mathrm{H} 3 \mathrm{~K} 9$ species could be rescued by overexpression of MDU. The authors concluded that MDU is one of the major histone H3K9 methyltransferases in the fly and produces mono-, di- and tri-methylated H3K9 in the third-instar larvae (Tzeng et al., 2007).

Another study investigated the role of MDU in Drosophila ovaries. In MDU mutant flies, $\mathrm{H} 3 \mathrm{~K} 9$ tri-methylation was absent throughout the germarium. The H3K9 di-methylation pattern did not change in the MDU mutant ovaries (Clough et al., 2007). It was concluded that MDU is required for tri-methylation, but not dimethylation, of histone $\mathrm{H} 3$ at the $\mathrm{K} 9$ residue. MDU mediates $\mathrm{H} 3 \mathrm{~K} 9$ tri-methylation in both the germ and somatic cells of the germarium and is the major HMT conferring H3K9 tri-methylation in these cells (Clough et al., 2007).

A second study dissecting the role of MDU in ovaries showed that MDU is responsible for the synthesis of H3K9 tri-methylation signals in the inner germarium, where GSCs (germline stem cells) and their early descendants are found. When these cells move to region-3 germarium, the H3K9 tri-methylation task is transferred to a combination of MDU and SU(VAR)3-9, because both enzymes act cooperatively in all other somatic-type cells of the germarium. After the egg chamber buds off from the germarium, the tri-methylation is regulated only by SU(VAR)3-9 (Yoon et al., 2008).

All three studies focused on the pattern of MDU-mediated H3K9 methylation at the chromatin level, but did not investigate histone methylation of MDU at specific target genes.

Our results suggest that MDU tri-methylates $\mathrm{H} 3 \mathrm{~K} 9$ at artificial and endogenous target genes in vivo. Tri-methylation of $\mathrm{H} 3 \mathrm{~K} 9$ by MDU triggers de novo DNA methylation. The different isoforms of MDU detected throughout development could establish different H3K9 methylation patterns. Our results support a model in which gene-specific tri-methylation of H3K9 plays an important role in gene silencing and de novo DNA methylation. 


\subsection{Interplay between DNA methylation and H3K9 tri-methylation; mechanism of MDU mediated $R b f$ silencing}

Methylation of DNA and histone H3K9 are among the best-characterized covalent modifications associated with silenced chromatin (Fuks 2005). In several organisms, methylation of H3K9 initiates DNA methylation, which suggests that an intricate interplay between the H3K9 and DNA methylation machineries plays an important role in chromatin structure and function. Studies of fungi, plants and mammals highlight methylation at lysine 9 of H3 as a kind of "beacon" for DNA methylation (Tamaru et al., 2001; Jackson et al., 2002; Lehnertz et al., 2003). In mammals, DNA methyltransferases interact with Suv39h H3K9 methyltransferases (Lehnertz et al., 2003; Fuks et al., 2003a), and loss of H3K9 methylation in Suv39h-knockout embryonic stem cells decreases Dnmt3b-dependent CpG methylation at major centromeric satellites (Lehnertz et al., 2003). In addition, H3K9 methylation and silencing of the p16ink4a tumor suppressor gene precedes CpG methylation (Bachman et al., 2003).

In Drosophila, the key players involved in methylation of $\mathrm{H} 3 \mathrm{~K} 9$ and DNA have been identified. However, whether and how DNA and H3K9 methylation machineries cooperate in the fly, in particular, how DNA methylation is initiated in the Drosophila genome, remained unknown.

The results of this study reveal that MDU plays an important role in de novo DNA methylation and gene silencing in Drosophila. The results of RT-PCR, XChIP and DNA methylation assays support the following model of how MDU directs DNA methylation and silencing of the tumor suppressor gene Rbf (Figure 38). 


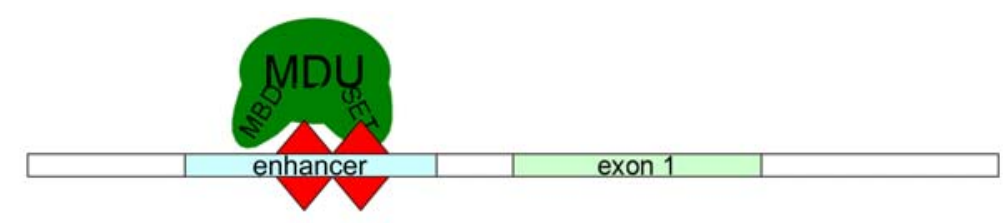

\section{A}

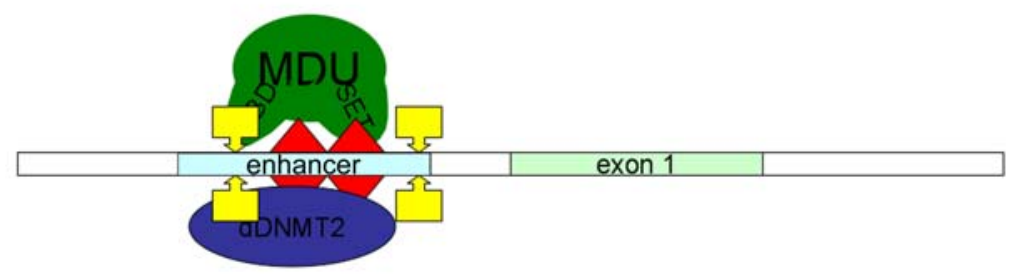

B
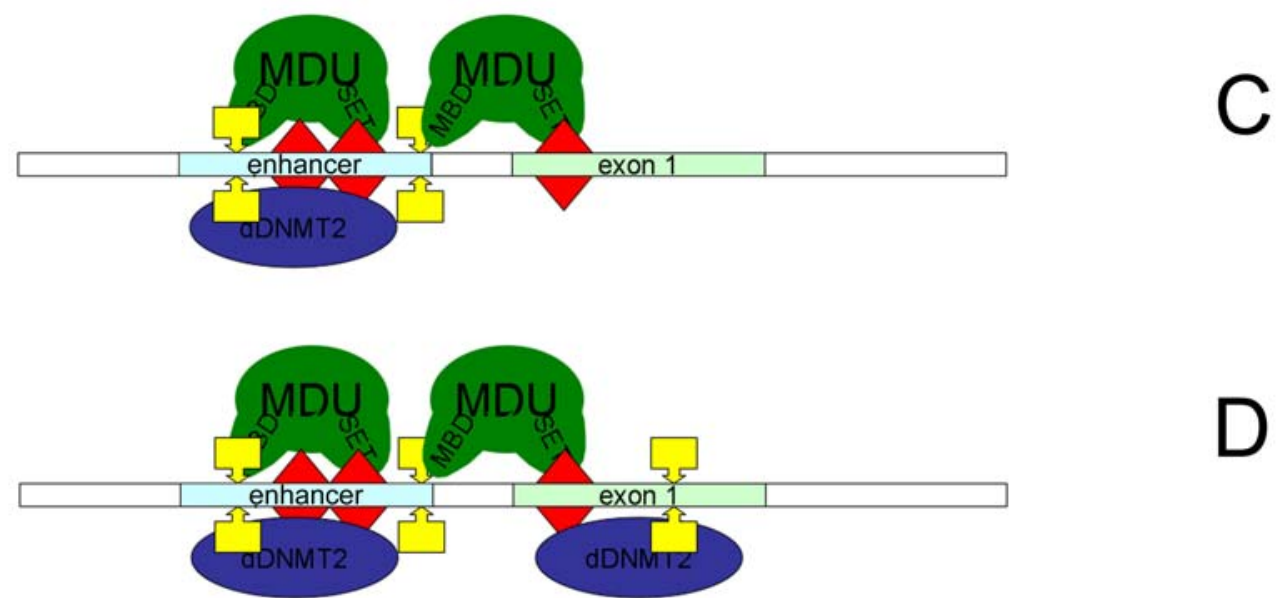

Figure 38. Establishment of repressive epigenetic modifications along the $R b f$ gene mediated by MDU. The model is illustrated in A, B, C, D pictures in the order of epigenetic events of the process. (A) Binding of MDU to the enhancer of Rbf leads to H3K9 tri-methylation. (B) H3K9 tri-methylation facilitates recruitment of dDNMT2. dDNMT2 catalyzes de novo DNA methylation in the enhancer of Rbf. (C) MDU binds methylated DNA via MBD domain, instigating reinitiation of $\mathrm{H} 3 \mathrm{~K} 9$ and DNA methylation, that results in spreading of the repressive signals from the enhacer to the exon 1 region. Spreading of $\mathrm{H} 3 \mathrm{~K} 9$ and DNA methylation results in silencing of $R b f$ transcription (D).

First, MDU is recruited to the enhancer region of Rbf. Once recruited, the SET domain of MDU tri-methylates $\mathrm{H} 3 \mathrm{~K} 9$ at the enhancer. Tri-methylation of $\mathrm{H} 3 \mathrm{~K} 9$ facilitates recruitment of dDNMT2, which results in DNA methylation. The observed attenuation of spreading of DNA and H3K9 methylation in cells expressing $\mathrm{MDU}(\mathrm{R} 436 \mathrm{C})$, which fails to bind methylated DNA, 
suggests that in the second phase, the MBD of MDU contacts methylated cytosines, which results in self-reinforced methylation of $\mathrm{H} 3 \mathrm{~K} 9$, recruitment of dDNMT2, and DNA methylation. The self-reinforcing and self-perpetuating mechanism results in spreading of H3K9 and DNA methylation from the enhancer to the exon 1 region and culminates in silencing of $R b f$ transcription. Both the SET and MBD domains of MDU play key roles in this model. SETmediated methylation of $\mathrm{H} 3 \mathrm{~K} 9$ triggers de novo DNA methylation; the MBD domain facilitates spreading of DNA and $\mathrm{H} 3 \mathrm{~K} 9$ methylation.

Recent models have proposed the existence of a self-reinforcing and self-perpetuating mechanism responsible for the spreading of DNA and/or histone methylation on target genes (Fuks 2005). Our findings provide the first experimental evidence for the existence of such a mechanism. Recent studies suggest that Su(var)3-9, a H3K9 specific HMT facilitates DNA methylation in Drosophila. Mutant alleles of Su(var)3-9 significantly decreased the level of genomic DNA methylation caused by over-expressing the mouse de novo methyltransferase Dnmt3a in Drosophila melanogaster (Weissmann et al., 2003), which supports our finding that DNA methylation in Drosophila depends on H3K9 methylation, as we demonstrate. Although the role of Su(var)3-9 in dDNMT2-mediated DNA methylation remains unclear, we propose that trimethylation of H3K9 by MDU and other HMTs such as Su(var)3-9 plays a key role in de novo DNA methylation in Drosophila.

However, several steps of the described model remain the topic of future investigations:

\section{How is MDU recruited to target genes?}

According to our model, repression of $R b f$ is initiated when MDU associates with the enhancer of Rbf. In the absence of MDU, the Rbf enhancer lacks tri-methylated H3K9 and DNA methylation, which indicates that these two modifications are not involved in the recruitment of MDU to target genes.

A recent study has associated the epigenetic repressors Pipsqueak (Psq) and Lola with repression of Rbf transcription in Drosophila (Ferres-Marco et al., 2006). The Lola gene encodes 25 alternatively spliced mRNAs that generate 19 different transcription factors. All of these transcription factors share a BTB domain (Broad complex, Tramtrack, Bric-a-brac, also known as POZ for Poxviruses zinc-finger). Psq encodes for four different proteins, three of which contain a BTB domain in the $\mathrm{NH}_{2}$-terminus. Furthermore, two of the BTB-containing isoforms and the isoform that lacks the BTB domain have four tandem copies of a conserved DNA-binding motif 
known as the Psq helix-turn-helix (HTH) motif (Ferres-Marco et al., 2006). The BTB domain is a protein-protein interaction motif that is found throughout eukaryotes. It determines a unique tridimensional fold with a large interaction surface. The exposed residues are highly variable and can permit dimerization and oligomerization, as well as interaction with a number of other proteins (Perez-Torrado et al., 2006).

The Psq-type HTH motif binds a GAGAG DNA sequence, which is present in many Polycomb-responsive elements of homeotic box (hox) genes, as well as at hundreds of other chromosomal sites (Schwendemann and Lehmann 2002). Psq is present in a multiprotein complex that displays PcG repressor and HDAC activity (Ringrose and Paro 2004). Thus, Psq and Lola could be required for sequence-specific targeting of Polycomb complexes to particular genes (Huang et al., 2002).

Psq and/or Lola could be involved in recruitment of MDU to Rbf. To test this possibility, one must confirm that binding of Lola and/or Psq precedes recruitment of MDU to Rbf and whether the factors facilitate recruitment of MDU to Rbf.

Recent studies revealed that non-coding RNAs could mediate the recruitment of epigenetic regulators to target DNA. In Drosophila, non-coding RNA transcribed from the trithorax response elements (TREs) of the homeotic gene Ultrabithorax (Ubx) facilitated recruitment of the epigenetic activator $\mathrm{ASH} 1$ to Ubx. In mammals, the non-coding RNA Hotair facilitates recruitment of the epigenetic repressor complex PRC2 to homeotic genes (Rinn et al., 2007). ASH1 binds TRE transcripts via the SET domain, and recent study implies that SET domains in general can bind single-stranded RNA and DNA (Krajewski et al., 2005). Because, like ASH1, MDU contains a SET domain, non-coding RNA may be involved in the recruitment of MDU to target genes. To test that hypothesis, one must investigate whether the MDU target genes such as Rbf produce non-coding RNA, which associates with MDU and facilitates recruitment of MDU.

\section{How does MDU-mediated tri-methylation facilitate recruitment of dDNMT2 to $R b f$ ?}

Our results suggest that tri-methylated $\mathrm{H} 3 \mathrm{~K} 9$ facilitates recruitment of dDNMT2 to target genes (Figure 30). dDNMT2 lacks the chromodomain, which is known to associate with tri-methylated H3K9, suggesting that dDNMT2 does not directly associate with tri-methylated H3K9.

Several studies revealed that HP1 family members can facilitate recruitment of DNMTs to methylated H3K9 (Smallwood et al., 2007; Honda and Selker 2008). Heterochromatin protein 1 (HP1) was first discovered in Drosophila as a dominant suppressor of position-effect variegation 
and a major component of heterochromatin (Clark and Elgin 1992). The $\mathrm{NH}_{2}$-terminal chromodomain of HP1 binds methylated lysine 9 of histone $\mathrm{H} 3$, causing transcriptional repression (Ayyanathan et al., 2003). The highly conserved $\mathrm{COOH}$-terminal chromoshadow domain enables dimerization and also serves as a docking site for proteins involved in a wide variety of nuclear functions, from transcription to nuclear architecture (Lechner et al., 2005). In addition to heterochromatin packaging, HP1 proteins are being increasingly considered to have diverse roles in the nucleus, including the regulation of euchromatic genes (Lomberk et al., 2006). The HP1 family is evolutionarily conserved, with members in fungi, plants, and animals, and multiple HP1 family members are expressed within the same species. DNMT1 interacts with HP1, which results in increased DNA methylation on DNA and chromatin templates in vitro (Smallwood et al., 2007). Loss of most of H3K9 methylation and HP1 binding on the fourth chromosome in MDU mutant flies was reported (Tzeng et al., 2007), which suggests that HP1 is recruited to the H3K9 methylation mediated by MDU. Thus, HP1 proteins may be involved in the recruitment of dDNMT2 to MDU-mediated tri-methylated H3K9. Further studies investigating the interaction between HP1 and dDNMT2 in vitro and in vivo are needed to uncover the role of HP1 in recruitment of dDNMT2 to MDU target genes.

\section{Why do DNA and H3K9 methylation spread from the enhancer to the exon region of $R b f$ ?}

MDU mediates spreading of H3K9 and DNA methylation from the enhancer to the exon region. The Rbf locus contains five CpG islands between the enhancer and first exon that protect the Rbf promoter from being methylated (Ferres-Marco et al., 2006). The CpG islands contain the CCGCGNGG motif, part of the consensus DNA binding site for the regulatory protein CTCF (Bell and Felsenfeld 2000). Originally identified as a transcriptional repressor, CTCF has since been characterized as a transcriptional activator, enhancer blocker, boundary definer and a potential genome organizer (Filippova 2008). The ability of CTCF to act with a diversity of functions has been attributed to its structure. CTCF contains 11 zinc-finger domains with which it binds DNA in a sequence-specific manner. Recently, CTCF was found to play a role in regulation of the transcription of non-coding RNA and establishing local chromatin structure at the repetitive elements in mammalian genomes (Filippova 2008).

DNA methylation prevents CTCF binding, and CTCF binding prevents CpG methylation. CTCF prevents spreading of DNA methylation and maintains methylation-free zones. The human homolog of $R b f$, the $R b$ gene, is subjected to epigenetic regulation by CTCF. CTCF has a protective role against epigenetic silencing by binding to the promoter of $R b$. Consistent with this, 
when the promoter is hypermethylated, CTCF binding is lost, and the site is recognized by the methyl-CpG-binding protein Kaiso (De La Rosa-Velázquez et al., 2007).

The CpG island (-1420 to -960) of Rbf located immediately downstream of the enhancer region, at which MDU initiates $\mathrm{H} 3 \mathrm{~K} 9$ and DNA methylation. It contains 14 repeats of the 33-bp core sequence TATTGACAAGGCAACCTCCGCGAGGAAATTGAC, each containing one CCGCGNGG motif of the DNA target sequence for CTCF. Upon recruitment of MDU, MDU facilitates spreading of DNA and H3K9 methylation, which results in methylation of CCGCGNGG motifs and removal of CTCF from the Rbf locus. Drosophila CTCF has been characterized only recently (Moon et al., 2005). The role of CTCF in the regulation of Rbf has not been demonstrated. Further investigation is needed to test this hypothesis.

\section{Are other factors involved in MDU-mediated silencing of gene expression?}

Our results suggest that the SET domain and MBD of MDU facilitate initiation and spreading of H3K9 and DNA methylation, which results in gene silencing. Whether proteins other than HP1 and dDNMT2 are involved in MDU-mediated repression is unclear. Numerous studies have demonstrated the role of MBD proteins in transcriptional repression involving the association of MBD proteins with an arsenal of various factors.

The canonical gene repression function of MeCP2 involves the molecule binding to methylated $\mathrm{CpG}$ sites via a conserved MBD, which leads to transcriptional repression due to recruitment of $\operatorname{Sin} 3 \mathrm{~A}$ and histone deacetylases (HDACs) and/or mediation of the methylation of histone H3 lysine 9 (Ballestar and Wolffe 2001b; Fuks et al., 2003a; Fuks et al., 2003b; Bienvenu and Chelly 2006).

The MBD protein MBD1 has also been reported to repress transcription in an HDACdependent way ( $\mathrm{Ng}$ et al., 2000). In addition, MBD1 can tether the Suv39h1-HP1 complex to methylated DNA regions (Fujita et al., 2003). The H3K9-specific HMTase Suv39h1 and the heterochromatin-binding protein HP1 directly interact with MBD of MBD1 in vitro and in cells. Suv39h1 was found to enhance MBD1-mediated transcriptional repression via MBD but not the $\mathrm{COOH}$-terminal transcriptional repression domain of MBD1. The association of MBD1 with histone deacetylases HDAC1/HDAC2 through Suv39h1 results in methylation and deacetylation of histones for gene inactivation (Fujita et al., 2003).

Despite the controversy whether Drosophila dMBD2/3 binds methylated DNA or not, dMBD2/3 has been associated with transcriptional repression (Roder et al., 2000; Ballestar et al., 2001a). However, these studies revealed different mechanisms of the dMBD2/3 mediated 
repression. In one study, repression by dMBD2/3 was suggested to be histone deacetylase dependent (Ballestar et al., 2001a). The other study showed that dMBD2/3 functions as transcription corepressor or repressors at unmethylated promoters in mammalian as well as Drosophila cells in a HDAC-independent fashion (Roder et al., 2000).

The obtained results suggest that the MBD of MDU mediates repression of $R b f$ by promoting the spreading of repressive epigenetic signals (DNA and histone methylation) and transcription repressors along the Rbf locus. However, although we cannot rule out that the MBD of MDU associates with other factors, which are involved in Rbf transcription, the observed attenuation of $R b f$ transcription in cells expressing MBD-inactive MDU suggests that the activity of the MBD of MDU in repression of $R b f$ transcription is primarily based on the ability of the MBD to bind methylated DNA rather than recruiting auxiliary factors.

\subsection{MDU and cell cycle regulation}

The Drosophila tumor suppressor protein RBF is involved in cell cycle progression, differentiation, apoptosis, and cell growth by controlling the transcription of key regulatory genes of those processes (Du et al., 1996a; Du et al., 1996b; Du and Dyson 1999). RBF is expressed uniformly in early embryo stage (Stevaux et al., 2002). Following germ band shortening, RBF protein is concentrated in the gut, epidermis, and developing CNS, with CNS expression continuing late into embryonic development (Keller et al., 2005). RBF represses transcription by interacting with the Drosophila DNA-binding E2F transcriptional factors dE2F1 and dE2F2, which directly recognize cognate promoter elements in responsive genes (Stevaux et al., 2002). RBF has also been suggested to control the rate of S-phase progression and has been implicated in control of cellular growth as opposed to proliferation (Xin et al., 2002).

RBF activity is regulated by changes in phosphorylation status mediated by cyclins $D$ and $E$ in association with cyclin-dependent kinases (Xin et al., 2002). RBF phosphorylation leads to release of E2F factors and relief of repression (Xin et al., 2002).

In addition to regulation at the protein level by phosphorylation, human $R b$ and Drosophila Rbf are subjected to epigenetic regulation. The 11-zinc finger CCCTC-binding factor (CTCF) is an epigenetic regulator of human $R b$ gene. CTCF has a protective role against epigenetic silencing by binding to the methylation-free promoter of $R b$ (De La Rosa-Velázquez et al., 2007). In Drosophila, Rbf expression is transcriptionally downregulated in the invasive tumors caused by elevated Notch signalling in combination with activation of components of the 
Polycomb complex of transcriptional repressors Lola and Psq (Ferres-Marco et al., 2006). Notably, the downregulation of Rbf in the Drosophila tumors is associated with increased DNA methylation of the $R b f$ locus. Furthermore, hypermethylation of $R b f$ is not simply the result of de novo transcription of dDNMT2 (Ferres-Marco et al., 2006). The same study suggested that $R b f$ transcription is controlled by epigenetic mechanisms involving methylation of DNA, H3K4, and H3K9 (Ferres-Marco et al., 2006).

The results of this study reveal that MDU plays a key role in silencing of Rbf. MDUmediated tri-methylation of H3K9 and DNA methylation result in silencing of Rbf expression. In the fly, RNAi of MDU in the developing eye results in abnormal cell proliferation/differentiation and generates a phenotype that resembles the phenotype in flies overexpressing RBF (Du et al., 1996a). These results imply that MDU regulates cell proliferation and cell differentiation by mediating the epigenetic silencing of RBF.

MDU's ortholog, human SETDB1, plays important roles in cell proliferation and differentiation. Takada et al. revealed that human SETDB1 participates in the non-canonical Wnt signaling and promotes osteoblastogenesis (the formation of osteoblasts) and represses adipogenesis (the formation of adipocytes) (Takada et al., 2007). Osteoblasts and adipocytes differentiate from common pleiotropic mesenchymal stem cells under transcriptional control by numerous factors and multiple intracellular signals (Ross et al., 2000). One of the key mediators of adipogenesis is the nuclear hormone receptor PPAR- $\gamma$ (Lehrke and Lazar 2005). On stimulation by its agonist ligand, PPAR- $\gamma$ enhances expression of target genes that force differentiation into adipocytes. The authors showed that Wnt5a (wingless-type MMTV integration site family, member 5A) activates Nemo-like kinase (NLK) through CaMKII (calcium/calmodulindependent protein kinase II alpha) and mitogen-activated protein (MAP) kinase kinase kinase TAK1/TAB2 signaling cascade. NLK phosphorylates SETDB1, leading to the formation of a chromatin-associated complex on target gene promoters. Complex formation is initiated by association of CHD7 (chromodomain helicase DNA binding protein 7) with DNA. CHD7 serves as a platform onto which ligand-bound PPAR- $\gamma$, NLK and SETDB1 asssemble. Recruitment of SETDB1 into the CHD7-SETDB1-PPAR- $\gamma$ complex leads to di- and tri-methylation of histone 3 at lysine 9 (H3K9) at PPAR- $\gamma$ target gene promoters, which leads to the silencing of PPAR- $\gamma$ target genes (Figure 39). 


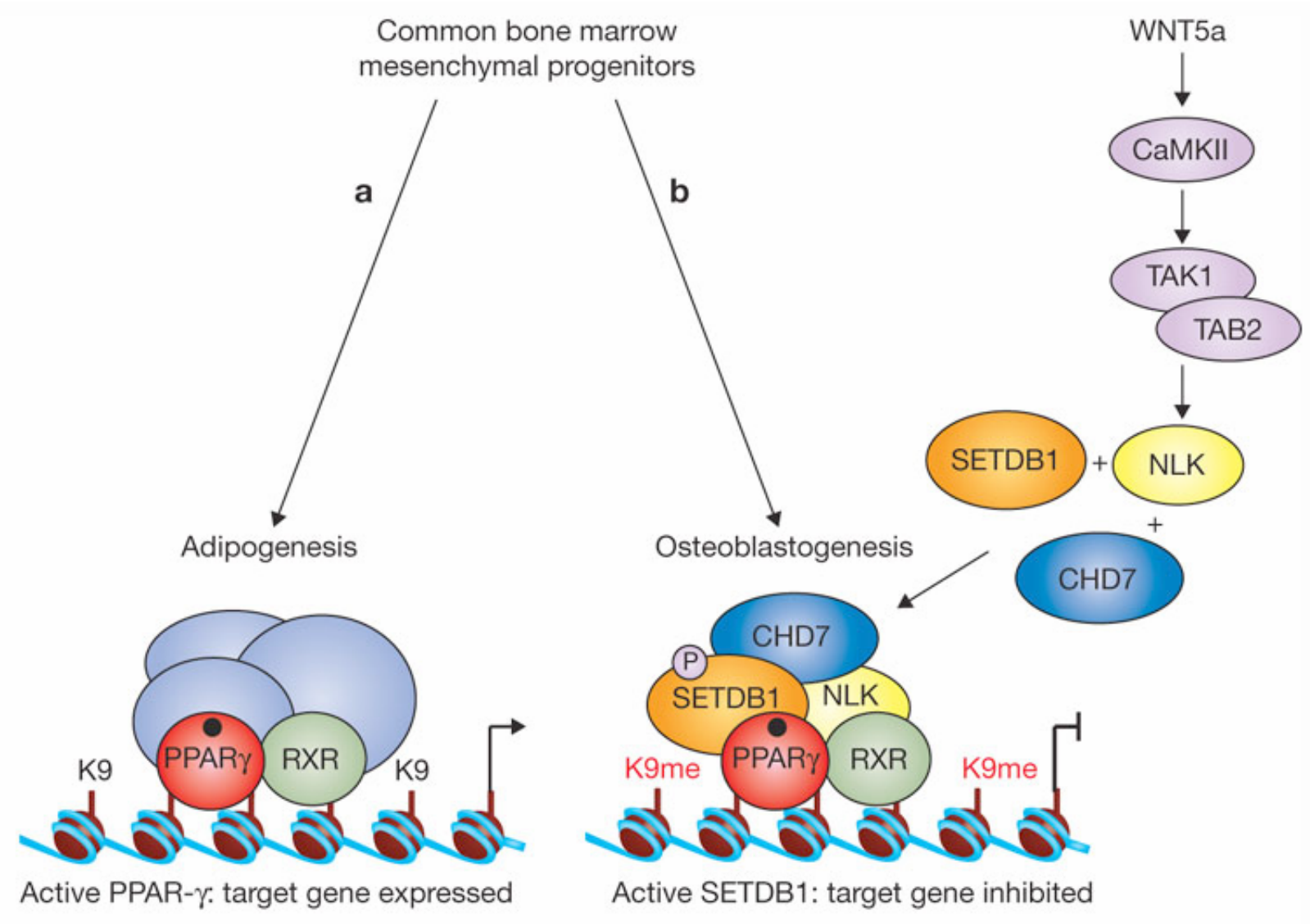

Figure 39. Role of SETDB1 in osteoblastogenesis mediated through a Noncanonical Wnt pathway. Expression of PPAR- $\gamma$ target genes (a) directs differentiation into adipocytes. Alternatively Wnt5a stimulates a non-canonical Wnt signalling cascade (b), which through CaMKII-TAK1/TAB2 results in activation of NLK. Consequently, phosphorylation of SETDB1 by activated NLK allows assembly of a SETDB1-NLK-CHD7 complex and activation of the histone methyltransferase activity of SETDB1. The SETDB1-NLK-CHD7 complex associated with ligand-bound PPAR- $\gamma$ to methylate $\mathrm{H} 3 \mathrm{~K} 9$ at the PPAR- $\gamma$ target gene, which results in gene silencing and directs cell differentiation into osteoblasts (Günther and Schüle 2007).

Our study suggested that MDU controls cell fate by regulating Rbf. Since Rbf transcription is regulated by the Notch signaling pathway (Ferres-Marco et al., 2006), the investigation of a functional connection between signal transduction pathways (for example, Notch pathway) and the epigenetic repressor MDU would be of interest. Further investigation of the relationships will help to clarify the mechanism of MDU-mediated cell fate determination.

The role of SETDB1 in noncanonical Wnt signal transduction pathway is through H3K9 di- or tri-methylation (Takada et al., 2007). Whether mammalian SET/MBD proteins can initiate de novo DNA methylation is unknown. We speculate that MBD/SET proteins play an important 
role in gene-specific de novo DNA methylation and gene silencing in Drosophila and other organisms. 


\section{Summary}

The methylation of genomic DNA and histones is paramount for the execution of epigenetic events such as imprinting, gene dosage compensation, and gene silencing. Studies in fungi, plants and vertebrates support a model in which histone methylation, in particular methylation of lysines 9 (H3K9) and/or 27 in histone H3, instigates de novo DNA methylation. However, little is known about the mechanisms mediating de novo DNA methylation in model organisms such as Drosophila melanogaster. Drosophila expresses key components of the DNA and histone machineries: a.) Histone methyltransferases of the SET-domain family (HMTs), which methylate H3K9 and play crucial roles in hetero-chromatin formation and maintenance and gene silencing; b.) One DNA methyltransferase (dDNMT2) which preferentially methylates CpA- and CpT-motifs in the Drosophila genome c.) Methyl-CpG binding domain (MBD) proteins, which potentially bind methylated DNA and convert DNA methylation into biological function. One member of the Drosophila MBD-protein family is MDU, which contains a MBD and SET-domain, which methylates $\mathrm{H} 3 \mathrm{~K} 9$ in vitro and in the fly, raising the possibility that MDU is involved in the H3K9 and DNA methylation machineries of the fly. In this thesis I have investigated the role of MDU in gene expression and DNA methylation. In vitro HMT-assays coupled Western blot and chromatin immunoprecipitation assays reveal that the SET-domain of MDU tri-methylates H3K9 in vitro and in vivo. MDU-mediated methylation of $\mathrm{H} 3 \mathrm{~K} 9$ facilitates trasncriptional repression of target genes in Drosophila cells, revealing that MDU mediates gene silencing. The MBD of MDU associates with methylated CpA-motifs in vitro, indicating that the MBD of MDU has intrinsic methyl DNA binding activity. Molecular and genetic data reveal that MDU represses the transcription of the tumor suppressor gene "retinoblastoma family protein" (Rbf), a key regulator of cell proliferation and differentiation. The dissection of the role of MDU in regulation of Rbf expression supports a model in which tri-methylation of H3K9 by MDU triggers dDNMT2-mediated de novo DNA methylation at an enhancer region of the Rbf locus. Once DNA methylation has been placed, the MBD of MDU associates with methylated DNA and induces a self-perpetuating histone-DNA methylation cascade that results in spreading of DNA and histone methylation along the $R b f$ locus and ultimately culminates in silencing of $R b f$ transcription. The obtained results uncover a role for the MBD/SET protein MDU in gene silencing, provide a mechanism for establishment of de novo DNA methylation in Drosophila, and imply that bifunctional MBD/SET proteins play important roles in the control of cell proliferation and differentiation in development. 


\section{Zusammenfassung}

Die Methylierung der genomischen DNA und Histonen im Kern eukaryotischer Zellen spielt eine wichtige Rolle in epigenetischen Prozessen wie z. B. Imprinting, “Gene Dosage Compensation”, und epigenetische Repression der Genexpression. Studien in Pilzen, Pflanzen und Vertebraten unterstützen ein Model, wonach die Methylierung von Histonen, insbesondere die Methylierung der Lysinreste 9 (H3K9) und/oder 27 im Histon 3, die de novo Methylierung von DNA auslöst. Im Gegensatz dazu, ist nur wenig über die Mechanismen bekannt, die für de novo Methylierung in Modelorganismen wie z. B. Drosophila melanogaster verantwortlich sind. Drosophila exprimiert Schlüsselfaktoren für die Methylierung von Histonen and DNA: a.) Histonmethyltransferasen (HMT), die H3K9 methylieren und entscheidende Rollen für die Etablierung and Aufrechterhaltung von Heterochromatin und Genrepression spielen; b.) Eine DNAMethyltransferase (dDNMT2), die überwiegend CpA und CpT-Motive im Drosophila Genom methyliert; c.) Methyl-CpG binding domain" (MBD) Proteine, die methylierte DNA binden und DNA Methylierung in biologische Aktivität übersetzen. Ein Mitglied der Drosophila MBD-Proteinfamilie ist Medusa (MDU), welches sowohl ein MBD- als auch ein SET-Motiv enthält. Das SET-Motiv methyliert H3K9 in vitro und In Drosophila. Die Anwesenheit eines MBD und Set Motivs in MDU unterstützt die Hypothese, dass MDU an der Methylierung von H3K9 und DNA in Drosophila beteiligt ist. In dieser Arbeit habe ich die funktionale Beduetung von MDU bezüglich der Genexpression and DNA Methylierung untersucht. In vitro HMTExperimente gekoppelt mit Western blot and Chromatin Immunpräzipitationsexperimenten ergaben, dass MDU H3K9 in vitro und in vivo tri-methyliert. MDU-vermittellte Methylierung von H3K9 resultiert in Repression der Zielgentranskription in Drosophila Zellkultur, woraus abgeleitet werden kann, dass MDU als Repressor der Transkription wirkt. Das MBD-Motiv von MDU bindet methylierte CpA DNA Sequenzen in vitro, und besitzt eine intrinsische "methylierte-DNA" Bindinggsaffinität. Die Ergebnisse molekularer and genetischer Studien zeigen, dass MDU die Transkription des Tumorsuppressor Gens "retinoblastoma family protein" (Rbf), einem Schlüsselregulator der Zellproliferation und -differenzierung, reprimiert. Die Untersuchungen zur Funktion von MDU in der Regulation der Expression von Rbf unterstützen ein Model wonach tri-methylierung von H3K9 durch MDU die dDNMT2-abhängige de novo Methylierung in der cisregulatorischen „enhancer“ Region von Rbf auslöst. Sobald DNA Methylierung platziert ist, bindet das MBD-Motif von MDU an methylierte DNA und induziert eine „selbstangetriebene“ DNA-Histone Mehtylierungskaskade, die zur Ausbreitung von DNA und H3K9 Methylierung auf dem Rbf Genlocus führt und letztendlich Repression der Rbf Transkription bewirkt. Die Ergebnisse dieser Arbeit entschlüsseln die Funktion von MDU in der Repression der Genexpression, ergeben einen Mechanismus für die Etablierung der de novo DNA Methylierung in Drosophila, und deuten auf eine wichtige Rolle der bifunktionalen MBD/SET Proteine für die Kontrolle der Proliferation und Differenzierung von Zellen hin. 


\section{References}

Aapola U, Kawasaki K, Scott HS, Ollila J, Vihinen M, Heino M, Shintani A, Kawasaki K, Minoshima S, Krohn K, Antonarakis SE, Shimizu N, Kudoh J, Peterson P. 2000. Isolation and initial characterization of a novel zinc finger gene, DNMT3L, on 21q22.3, related to the cytosine-5-methyltransferase 3 gene family. Genomics 65: 293-298.

Adams RLP. 1996. DNA methylation . In Principles of Medical Biology, Vol. 5 ( Bittar, E.E. , ed.) , pp. 33- 66.

Ahn SH, Henderson KA, Keeney S \& Allis CD. 2005. H2B (Ser10) phosphorylation is induced during apoptosis and meiosis in S. cerevisiae. Cell Cycle 4, 780-783.

Alberts B, Bray D, Hopkin K, Johnson A, Lewis J, Raff M, Roberts K, Walter P. 2004. Essential Cell Biology, Second Edition, published by Garland Science in 2004.

Allfrey VG, Faulkner R, Mirsky AE. 1964. Acetylation and methylation of histones and their possible role in the regulation of RNA synthesis. Proc. Natl. Acad. Sci. USA 51: 786-94.

Amir RE, Van den Veyver IB, Wan M, Tran CQ, Francke U, Zoghbi HY. 1999. Rett syndrome is caused by mutations in X-linked MECP2, encoding methyl-CpG-binding protein 2. Nat Genet. 1999 Oct;23(2):185-8.

Anastassiadis K, Kim J, Daigle N, Sprengel R, Schöler HR, Stewart AF. 2002. A predictable ligand regulated expression strategy for stably integrated transgenes in mammalian cells in culture. Gene. 2002 Oct 2;298(2):159-72.

Antequera F, Boyes J, and Bird A. 1990. High levels of de novo methylation and altered chromatin structure at CpG islands in cell lines. Cell 62: 503-514

Arents G, Moudrianakis EN. 1995. The histone fold: a ubiquitous architectural motif utilized in DNA compaction and protein dimerization. Proc Natl Acad Sci USA. 92(24):1117011174.

Asano M, Nevins JR, Wharton RP. 1996. Ectopic E2F expression induces S phase and apoptosis in Drosophila imaginal discs. Genes Dev. 1996 Jun 1;10(11):1422-32.

Attwooll C, Lazzerini Denchi E, Helin K. 2004. The E2F family: specific functions and overlapping interests. EMBO J. 2004 Dec 8;23(24):4709-16.

Ayyanathan K, Lechner MS, Bell P, Maul GG, Schultz DC, Yamada Y, Tanaka K, Torigoe K, Rauscher FJ 3rd. 2003. Regulated recruitment of HP1 to a euchromatic gene induces mitotically heritable, epigenetic gene silencing: a mammalian cell culture model of gene variegation. Genes Dev. 17, 1855-1869.

Baarends WM, Hoogerbrugge JW, Roest HP, Ooms M, Vreeburg J, Hoeijmakers JH and Grootegoed JA. 1999. Histone ubiquitination and chromatin remodeling in mouse spermatogenesis. Dev. Biol. 207: 322-333.

Babu A and Verma RS. 1987. Chromosome structure: euchromatin and heterochromatin. Int Rev Cytol. 1987;108:1-60.

Bachman KE, Park BH, Rhee I, Rajagopalan H, Herman JG, Baylin SB, Kinzler KW, Vogelstein B. 2003. Histone modifications and silencing prior to DNA methylation of a tumor suppressor gene. Cancer Cell. 2003 Jan;3(1):89-95.

Ballestar E, Pile LA, Wassarman DA, Wolffe AP, Wade PA. 2001a. A Drosophila MBD family member is a transcriptional co-repressor associated with specific genes. Eur. J. Biochem. 268 (2001), pp. 5397-5406.

Ballestar E and Wolffe AP. 2001b. Methyl-CpG-binding proteins: targeting specific gene repression, Eur. J. Biochem. 268 (2001), pp. 1-6.

Bannister AJ and Kouzarides T. 2004. Histone methylation: recognizing the methyl mark, Methods Enzymol. 376 (2004), pp. 269-288. 
Barber CM, Turner FB, Wang Y, Hagstrom K, Taverna SD, Mollah S, Ueberheide B, Meyer BJ, Hunt DF, Cheung P, Allis CD. 2004. The enhancement of histone $\mathrm{H} 4$ and $\mathrm{H} 2 \mathrm{~A}$ serine 1 phosphorylation during mitosis and S-phase is evolutionarily conserved. Chromosoma 112, 360-371.

Baxevanis AD, Arents G, Moudrianakis EN, Landsman D. 1995. A variety of DNA-binding and multimeric proteins contain the histone fold motif. Nucleic Acids Res. 23(14):26852691.

Baylin SB and Herman JG. 2000. DNA hypermethylation in tumorigenesis: Epigenetics joins genetics. Trends Genet. 16: 168-174

Bedford MT and Richard S. 2005. Arginine methylation an emerging regulator of protein function. Mol. Cell 18, 263-272.

Beisel C, Imhof A, Greene J, Kremmer E, Sauer F. 2002. Histone methylation by the Drosophila epigenetic transcriptional regulator Ash1.Nature. 2002 Oct 24;419(6909):85762.

Bell AC and Felsenfeld G. 2000. Methylation of a CTCF-dependent boundary controls imprinted expression of the lgf2 gene. Nature 405: 482-485

Berger SL. 2002. Histone modifications in transcriptional regulation. Curr. Opin. Genet. Dev. 12(2): 142-8.

Bestor T, Laudano A, Mattaliano R and Ingram V. 1988. Cloning and sequencing of a cDNA encoding DNA methyltransferase of mouse cells. The carboxyl-terminal domain of the mammalian enzymes is related to bacterial restriction methyltransferases. J. Mol. Biol. 203 (1988), pp. 971-983.

Bestor TH. 2000. The DNA methyltransferases of mammals. Hum Mol Genet 9: 2395-2402.

Bienvenu T, Chelly J. 2006. Molecular genetics of Rett syndrome: when DNA methylation goes unrecognized. Nat. Rev. Genet. 7, 415-426.

Bird A. 2002. DNA methylation patterns and epigenetic memory. Genes Dev. 1;16(1):6-21.

Bird A. 2007. Perceptions of epigenetics. Nature. 2007 May 24;447(7143):396-8.

Bird AP and Wolffe A.P., 1999. Methylation-induced repression - belts, braces, and chromatin, Cell 99 (1999), pp. 451-454.

Bird AP, Taggart MH, and Smith BA. 1979. Methylated and unmethylated DNA compartments in the sea urchin genome. Cell 17: 889-901

Bird AP. 1980. DNA methylation and the frequency of CpG in animal DNA. Nucleic Acids Res. 8: 1499-1594.

Bourc'his D, Xu GL, Lin CS, Bollman B, Bestor TH. 2001. Dnmt3L and the establishment of maternal genomic imprints. Science 294: 2536-2539.

Bowen NJ, Palmer MB, Wade PA. 2004. Chromosomal regulation by MeCP2: structural and enzymatic considerations. Cell Mol Life Sci. 2004 Sep;61(17):2163-7.

Bradbury EM. 1992. Reversible histone modifications and the chromosome cell cycle. Bioessays 14, 9-16.

Brand AH, Perrimon N. 1993. Targeted gene expression as a means of altering cell fates and generating dominant phenotypes. Development. 1993 Jun;118(2):401-15.

Brehm A, Miska EA, McCance DJ, Reid JL, Bannister AJ, Kouzarides T. 1998. Retinoblastoma protein recruits histone deacetylase to repress transcription. Nature. 1998 Feb 5;391(6667):597-601.

Brower-Toland B, Wacker DA, Fulbright RM, Lis JT, Kraus WL, Wang MD. 2005. Specific contributions of histone tails and their acetylation to the mechanical stability of nucleosomes. J Mol Biol. 11;346(1):135-46

Bullock WO, Fernandez JM, Short JM. 1987. XL1-Blue: A high efficiency plasmid transforming recA Escherichia coli strain with beta-galactosidase selection. Biotechniques. $1987 \mathrm{Vol}$. 5, no. 4, pp. 376-379.

Cao X, Jacobsen SE. 2002. Role of the arabidopsis DRM methyltransferases in de novo DNA 
methylation and gene silencing. Curr Biol. 2002 Jul 9;12(13):1138-44.

Cao X, Springer NM, Muszynski MG, Phillips RL, Kaeppler S, Jacobsen SE. 2000. Conserved plant genes with similarity to mammalian de novo DNA methyltransferases. Proc Natl Acad Sci U S A. 2000 Apr 25;97(9):4979-84.

Capotosti F, Hsieh JJ, Herr W. 2007. Species selectivity of mixed-lineage leukemia/trithorax and HCF proteolytic maturation pathways. Mol Cell Biol. 2007 Oct;27(20):7063-72.

Chakravarthy S, Park YJ, Chodaparambil J, Edayathumangalam RS, Luger K. 2005. Structure and dynamic properties of nucleosome core particles. FEBS Lett. 579(4): 8958.

Chang B, Chen Y, Zhao Y, Bruick RK. 2007. JMJD6 is a histone arginine demethylase. Science. 2007 Oct 19;318(5849):444-7.

Chellappan SP, Hiebert S, Mudryj M, Horowitz JM, Nevins JR. 1991. The E2F transcription factor is a cellular target for the RB protein. Cell. 1991 Jun 14;65(6):1053-61.

Chen D, Dundr M, Wang C, Leung A, Lamond A, Misteli T, Huang S. 2005. Condensed mitotic chromatin is accessible to transcription factors and chromatin structural proteins. J. Cell Biol. 168(1): 41-54.

Chen HY, Sun JM, Zhang Y, Davie R, and Meistrich ML. 1998. Ubiquitination of histone H3 in elongating spermatids of rat testes. J. Biol. Chem. 273: 13165-13169.

Chuikov S, Kurash JK, Wilson JR, Xiao B, Justin N, Ivanov GS, McKinney K, Tempst P, Prives C, Gamblin SJ, Barlev NA, Reinberg D. 2004. Regulation of p53 activity through lysine methylation, Nature 432 (2004), pp. 353-360.

Clark RF and Elgin SC. 1992. Heterochromatin protein 1, a known suppressor of position-effect variegation, is highly conserved in Drosophila. Nucleic Acids Res. 1992 Nov 25;20(22):6067-74.

Clayton AL, Hazzalin CA, Mahadevan LC. 2006. Enhanced histone acetylation and transcription: a dynamic perspective. Mol Cell. 23(3):289-96.

Clough E, Moon W, Wang S, Smith K, Hazelrigg T. 2007. Histone methylation is required for oogenesis in Drosophila. Development. 2007 Jan;134(1):157-65.

Colin Dingwall and Ronald A. Laskey. 1991. Nuclear targeting sequences - a consensus? Trends in Biochemical Sciences Volume 16, 1991, Pages 478-481.

Colot V and Rossignol JL. 1999. Eukaryotic DNA methylation as an evolutionary device. BioEssays 21: 402-411

Cosgrove MS, Boeke JD, Wolberger C. 2004. Regulated nucleosome mobility and the histone code. Nat. Struct. Mol. Biol. 11(11): 1037-43. Review.

Cross SH, Meehan RR, Nan X, and Bird A. 1997. A component of the transcriptional repressor MeCP1 is related to mammalian DNA methyltransferase and trithorax-like protein. Nat. Genet. 16: 256-259.

Dannenberg JH and te Riele HP. 2006. The retinoblastoma gene family in cell cycle regulation and suppression of tumorigenesis. Results Probl Cell Differ. 2006;42:183-225.

Davie JR. and Chadee DN. 1998. Regulation and regulatory parameters of histone modifications, J. Cell. Biochem. Suppl. 30-31, pp. 203-213.

Dawson BA, Herman T, Haas AL, and Lough J. 1991. Affinity isolation of active murine erythroleukemia cell chromatin: Uniform distribution of ubiquitinated histone $\mathrm{H} 2 \mathrm{~A}$ between active and inactive fractions. J. Cell. Biochem. 46: 166-173.

De La Rosa-Velázquez IA, Rincón-Arano H, Benítez-Bribiesca L, Recillas-Targa F. 2007. Epigenetic regulation of the human retinoblastoma tumor suppressor gene promoter by CTCF. Cancer Res. 2007 Mar 15;67(6):2577-85.

De Luca A, MacLachlan TK, Bagella L, Dean C, Howard CM, Claudio PP, Baldi A, Khalili K, Giordano A. 1997. A unique domain of pRb2/p130 acts as an inhibitor of Cdk2 kinase activity. J Biol Chem. 1997 Aug 22;272(34):20971-4. 
De Nooij JC, Letendre MA, Hariharan IK. 1996. A cyclin-dependent kinase inhibitor, Dacapo, is necessary for timely exit from the cell cycle during Drosophila embryogenesis. Cell. 1996 Dec 27;87(7):1237-47.

Dillon SC, Zhang X, Trievel RC, Cheng X. 2005. The SET-domain protein superfamily: protein lysine methyltransferases. Genome Biol. 2005;6(8):227.

Dobosy JR and Selker EU. 2001. Emerging connections between DNA methylation and histone acetylation, Cell. Mol. Life Sci. 58 (2001), pp. 721-727.

Dominguez M. 2006. Interplay between Notch signaling and epigenetic silencers in cancer. Cancer Res. 2006 Sep 15;66(18):8931-4.

Dowdy SF, Hinds PW, Louie K, Reed SI, Arnold A, Weinberg RA. 1993. Physical interaction of the retinoblastoma protein with human D cyclins. Cell. 1993 May 7;73(3):499-511.

Du W and Dyson N. 1999. The role of RBF in the introduction of $\mathrm{G} 1$ regulation during Drosophila embryogenesis. EMBO J. 1999 Feb 15;18(4):916-25.

Du W, Vidal M, Xie JE, Dyson N. 1996a. RBF, a novel RB-related gene that regulates E2F activity and interacts with cyclin E in Drosophila. Genes Dev. 1996 May 15;10(10):120618.

Du W, Xie JE, Dyson N. 1996b. Ectopic expression of dE2F and dDP induces cell proliferation and death in the Drosophila eye. EMBO J. 1996 Jul 15;15(14):3684-92.

Duncan BK and Miller JH. 1980. Mutagenic deamination of cytosine residues in DNA. Nature 287: 560-561.

Dynlacht BD, Brook A, Dembski M, Yenush L, Dyson N. 1994. DNA-binding and transactivation properties of Drosophila E2F and DP proteins. Proc Natl Acad Sci U S A. 1994 Jul 5;91(14):6359-63.

Dyson MH, Rose S and Mahadevan LC. 2001. Acetyllysine-binding and function of bromodomain-containing proteins in chromatin, Front. Biosci. 6 (2001), pp. D853-D865.

Dyson N. 1998. The regulation of E2F by pRB-family proteins. Genes Dev. 1998 Aug 1;12(15):2245-62.

Ebert A, Lein S, Schotta G, Reuter G. 2006. Histone modification and the control of heterochromatic gene silencing in Drosophila. Chromosome Res. 2006;14(4):377-92.

Endicott JA, Noble ME. 1998. Structural principles in cell-cycle control: beyond the CDKs. Structure. 1998 May 15;6(5):535-41.

Efthymiadis A, Shao H, Hübner S, Jans DA. 1997. Kinetic characterization of the human retinoblastoma protein bipartite nuclear localization sequence (NLS) in vivo and in vitro. A comparison with the SV40 large T-antigen NLS. J Biol Chem. 1997 Aug 29;272(35):22134-9.

Ehrenhofer-Murray AE. 2004. Chromatin dynamics at DNA replication, transcription and repair. Eur. J. Biochem. 271, 2335-2349.

Ehrlich K. 1993. Characterization of DBPm, a plant protein that binds to DNA containing 5methylcytosine. Biochim. Biophys. Acta 1172: 108-116.

Ewen ME, Sluss HK, Sherr CJ, Matsushime H, Kato J, Livingston DM. 1993. Functional interactions of the retinoblastoma protein with mammalian D-type cyclins. Cell. 1993 May 7;73(3):487-97.

Fang J, Chen T, Chadwick B, Li E and Zhang Y. 2004. Ring1b-mediated H2A ubiquitination associates with inactive $X$ chromosomes and is involved in initiation of $X$ inactivation. J. Biol. Chem. 279, 52812-52815.

Fang J, Feng Q, Ketel CS, Wang H, Cao R, Xia L, Erdjument-Bromage H, Tempst P, Simon JA. and Zhang Y. 2002. Purification and functional characterization of SET8, a nucleosomal histone H4-lysine 20-specific methyltransferase, Curr Biol 12 (2002), pp. 1086-1099. 
Ferres-Marco D, Gutierrez-Garcia I, Vallejo DM, Bolivar J, Gutierrez-Aviño FJ, Dominguez M. 2006. Epigenetic silencers and Notch collaborate to promote malignant tumours by Rb silencing. Nature. 2006 Jan 26;439(7075):430-6.

Filion GJ, Zhenilo S, Salozhin S, Yamada D, Prokhortchouk E and Defossez PA. 2006. A family of human zinc finger proteins that bind methylated DNA and repress transcription, Mol. Cell. Biol. 26 (2006), pp. 169-181.

Filippova GN. 2008. Genetics and epigenetics of the multifunctional protein CTCF. Curr Top Dev Biol. 2008;80:337-60.

Finnegan EJ, Dennis ES. 1993. Isolation and identification by sequence homology of a putative cytosine methyltransferase from Arabidopsis thaliana. Nucleic Acids Res. 1993 May 25;21(10):2383-8.

Fisher O, Siman-Tov R, Ankri S. 2004. Characterization of cytosine methylated regions and 5cytosine DNA methyltransferase (Ehmeth) in the protozoan parasite Entamoeba histolytica. Nucleic Acids Res. 2004;32:287-297.

Foster ER \& Downs JA. 2005. Histone H2A phosphorylation in DNA double-strand break repair. FEBS J. 272, 3231-3240.

Fraga MF, Esteller M. 2002. DNA methylation: a profile of methods and applications. Biotechniques. 2002 Sep;33(3):632, 634, 636-49.

Free A, Wakefield RI, Smith BO, Dryden DT, Barlow PN, Bird AP. 2001. DNA recognition by the methyl-CpG binding domain of MeCP2. J Biol Chem. 2001 Feb 2;276(5):3353-60.

Freitas MA, Sklenar AR, Parthun MR. 2004. Application of mass spectrometry to the identification and quantification of histone post-translational modifications. J. Cell Biochem. 92(4): 691-700.

Frolov MV, Huen DS, Stevaux O, Dimova D, Balczarek-Strang K, Elsdon M, Dyson NJ. 2001. Functional antagonism between E2F family members. Genes Dev. 2001 Aug 15;15(16):2146-60.

Fujita N, Watanabe S, Ichimura T, Tsuruzoe S, Shinkai Y, Tachibana M, Chiba T, Nakao M. 2003. Methyl-CpG binding domain 1 (MBD1) interacts with the Suv39h1-HP1 heterochromatic complex for DNA methylation-based transcriptional repression. J Biol Chem. 2003 Jun 27;278(26):24132-8.

Fuks F, Hurd PJ, Deplus R, Kouzarides T. 2003a. The DNA methyltransferases associate with HP1 and the SUV39H1 histone methyltransferase. Nucleic Acids Res. 31, 2305-2312.

Fuks F, Hurd PJ, Wolf D, Nan X, Bird AP, Kouzarides T. 2003b. The methyl-CpG-binding protein MeCP2 links DNA methylation to histone methylation. J. Biol. Chem. 278, 40354040.

Fuks F. 2005. DNA methylation and histone modifications: teaming up to silence genes. Curr Opin Genet Dev. 2005 Oct;15(5):490-5.

Georgel PT, Horowitz-Scherer RA, Adkins N, Woodcock CL, Wade PA and Hansen JC. 2003. Chromatin compaction by human MeCP2. Assembly of novel secondary chromatin structures in the absence of DNA methylation. J. Biol. Chem. 278: 32181-32188

Ghosh RP, Horowitz-Scherer RA, Nikitina T, Gierasch LM, Woodcock CL. 2008. Rett syndrome-causing mutations in human MECP2 result in diverse structural changes that impact folding and DNA interactions. J Biol Chem. 2008 May 22.

Goldknopf IL, Taylor CW, Baum RM, Yeoman LC, Olson MO, Prestayko AW and Busch H. 1975. Isolation and characterization of protein A24, a "istone-like" nonhistone chromosomal protein. J. Biol. Chem. 250: 7182-7187.

Goll MG, Kirpekar F, Maggert KA, Yoder JA, Hsieh CL, Zhang X, Golic KG, Jacobsen SE, Bestor TH. 2006. Methylation of tRNAAsp by the DNA methyltransferase homolog Dnmt2. Science. 2006;311:395-398.

Gossen M, Bujard H. 1992. Tight control of gene expression in mammalian cells by tetracycline-responsive promoters. Proc Natl Acad Sci U S A. 1992 Jun 15;89(12):5547- 
51.

Gowher H, Leismann O, Jeltsch A. 2000. DNA of Drosophila melanogaster contains 5methylcytosine. EMBO J. 2000 Dec 15;19(24):6918-23.

Greger V, Passarge E, Hopping W, Messmer E \& Horsthemke B. 1989. Epigenetic changes may contribute to the formation and spontaneous regression of retinoblastoma. Hum. Genet. 83, 155-158.

Grewal SI \& Moazed D. 2003. Heterochromatin and epigenetic control of gene expression. Science 301, 798-802.

Gruenbaum Y, Naveh-Many T, Cedar H and Razin A. 1981. Sequence specificity of methylation in higher plant DNA, Nature 292 (1981), pp. 860-862.

Grunstein M. 1997. Histone acetylation in chromatin structure and transcription. Nature 389: 349-52.

Günther T and Schüle R. 2007. Fat or bone? A non-canonical decision. Nat Cell Biol. 2007 Nov;9(11):1229-31.

Hansen JC. 2002. Conformational dynamics of the chromatin fiber in solution: determinants, mechanisms, and functions. Annu. Rev. Biophys. Biomol. Struct. 31: 361-92.

Hansen RS. 2003. $X$ inactivation-specific methylation of LINE-1 elements by DNMT3B: implications for the Lyon repeat hypothesis. Hum Mol Genet. 2003 Oct 1;12(19):2559-67.

Hark AT, Schoenherr CJ, Katz DJ, Ingram RS, Levorse JM, and Tilghman SM. 2000. CTCF mediates methylation-sensitive enhancer-blocking activity at the H19/lgf2 locus. Nature 405: 486-489.

Harlow E. and Lane D. 1999. Antibodies: A Laboratory Manual. Cold Spring Harbour Laboratory Press. (ISBN 0879695439)

Harris M. 1982. Induction of thymidine kinase in enzyme-deficient Chinese hamster cells. Cell 29: 483-492.

Hata K, Okano M, Lei H, Li E. 2002. Dnmt3L cooperates with the Dnmt3 family of de novo DNA methyltransferases to establish maternal imprints in mice. Development. 2002 Apr;129(8):1983-93.

Hazzalin CA and Mahadevan LC. 2005. Dynamic acetylation of all lysine 4-methylated histone $\mathrm{H} 3$ in the mouse nucleus: analysis at c-fos and c-jun, PLoS Biol. 3 p. e393.

Heitmann B, Maurer T, Weitzel JM, Strätling WH, Kalbitzer HR, Brunner E. 2003. Solution structure of the matrix attachment region-binding domain of chicken MeCP2. Eur. J. Biochem. 270, 3263-3270.

Hendrich B and Bird A. 1998. Identification and characterization of a family of mammalian methyl-CpG binding proteins. Mol. Cell. Biol. 18: 6538-6547.

Hendrich B and Tweedie S. 2003. The methyl-CpG binding domain and the evolving role of DNA methylation in animals, Trends Genet. 19 (2003), pp. 269-277.

Henikoff S and Comai L. 1998. A DNA methyltransferase homolog with a chromodomain exists in multiple polymorphic forms in Arabidopsis. Genetics 149: 307-318.

Herceg $Z$ and Wang ZQ. 2005. Rendez-vous at mitosis: TRRAPed in the chromatin, Cell Cycle 4 (2005), pp. 383-387.

Hermann A, Gowher H, Jeltsch A. 2004. Biochemistry and biology of mammalian DNA methyltransferases. Cell Mol Life Sci 61: 2571-2587.

Hermann A, Schmitt S, Jeltsch A. 2003. The human Dnmt2 has residual DNA-(cytosine-C5) methyltransferase activity. J Biol Chem. 2003;278:31717-31721.

Hernando E, Nahlé Z, Juan G, Diaz-Rodriguez E, Alaminos M, Hemann M, Michel L, Mittal V, Gerald W, Benezra R, Lowe SW, Cordon-Cardo C. 2004. Rb inactivation promotes genomic instability by uncoupling cell cycle progression from mitotic control. Nature. 2004 Aug 12;430(7001):797-802. 
Hinds PW, Mittnacht S, Dulic V, Arnold A, Reed SI, Weinberg RA. 1992. Regulation of retinoblastoma protein functions by ectopic expression of human cyclins. Cell. 1992 Sep 18;70(6):993-1006.

Ho KL, McNae IW, Schmiedeberg L, Klose RJ, Bird AP, Walkinshaw MD. 2008. MeCP2 binding to DNA depends upon hydration at methyl-CpG. Mol Cell. 2008 Feb 29;29(4):525-31.

Holmgren C, Kanduri C, Dell G, Ward A, Mukhopadhya R, Kanduri M, Lobanenkov V, and Ohlsson R. 2001. CpG methylation regulates the lgf2/H19 insulator. Curr. Biol. 11: 11281130.

Honda S and Selker EU. 2008. Direct interaction between DNA methyltransferase DIM-2 and HP1 is required for DNA methylation in Neurospora. Mol Cell Biol. 2008 Aug 4.

Horn PJ, Peterson CL. 2002. Molecular biology. Chromatin higher order folding--wrapping up transcription. Science 297: 1824-7.

Hsieh JJ, Cheng EH, Korsmeyer SJ. 2003. Taspase1: a threonine aspartase required for cleavage of MLL and proper HOX gene expression. Cell. 2003 Oct 31;115(3):293-303.

Hsieh CL. 2005 The de novo methylation activity of Dnmt3a is distinctly different than that of Dnmt1. BMC Biochem 6: 6.

Huang DH, Chang YL, Yang CC, Pan IC, King B. 2002. pipsqueak encodes a factor essential for sequence-specific targeting of a polycomb group protein complex. Mol Cell Biol. 2002 Sep;22(17):6261-71.

Huang SY, Barnard MB, Xu M, Matsui S, Rose SM, and Garrard WT. 1986. The active immunoglobulin kappa chain gene is packaged by non-ubiquitin-conjugated nucleosomes. Proc. Natl. Acad. Sci. 83: 3738-3742.

Hung MS, Karthikeyan N, Huang B, Koo HC, Kiger J and Shen CJ. 1999. Drosophila proteins related to vertebrate DNA (5-cytosine) methyltransferases. Proc. Natl. Acad. Sci. 96: 11940-11945.

Inoue H, Nojima H, Okayama H. 1990. High efficiency transformation of Escherichia coli with plasmids. Gene 96(1):23-8.

Issa JP. 2000. CpG-island methylation in aging and cancer. Curr. Top. Microbiol. Immunol. 249: 101-118.

Ito M, Koike A, Koizumi N, Sano H. 2003. Methylated DNA-binding proteins from Arabidopsis, Plant Physiol. 133 (2003), pp. 1747-1754.

Ito T. 2007. Role of histone modification in chromatin dynamics. J Biochem. 141(5):609-14.

Jackson JP, Johnson L, Jasencakova Z, Zhang X, PerezBurgos L, Singh PB, Cheng X, Schubert I, Jenuwein T, Jacobsen SE. 2004. Dimethylation of histone H3 lysine 9 is a critical mark for DNA methylation and gene silencing in Arabidopsis thaliana. Chromosoma. 2004;112:308-315.

Jackson JP, Lindroth AM, Cao X, Jacobsen SE. 2002. Control of CpNpG DNA methylation by the KRYPTONITE histone H3 methyltransferase. Nature. 2002;416:556-560.

Jacobs SA, Khorasanizadeh S. 2002. Structure of HP1 chromodomain bound to a lysine 9methylated histone H3 tail. Science. 2002 Mar 15;295(5562):2080-3.

Jaenisch R, Harbers K, Jahner D, Stewart C, and Stuhlmann H. 1982. DNA methylation, retroviruses, and embryogenesis. J. Cell Biochem. 20: 331-336.

Jason LJ, Moore SC, Lewis JD, Lindsey G, and Ausio J. 2002. Histone ubiquitination: A tagging tail unfolds? BioEssays 24: 166-174.

Jeltsch A, Nellen W, Lyko F. 2006. Two substrates are better than one: dual specificities for Dnmt2 methyltransferases. Trends Biochem Sci. 2006;31:306-308.

Jenuwein T, Allis CD. 2001. Translating the histone code. Science. 293(5532):1074-1080.

Jones PA, Wolkowicz MJ, Rideout WMI, Gonzales FA, Marziasz CM, Coetzee GA, and Tapscott SJ. 1990. De novo methylation of the MyoD1 CpG island during the establishment of immortal cell lines. Proc. Natl. Acad. Sci. 87: 6117-6121. 
Jones RS, Gelbart WM. 1993. The Drosophila Polycomb-group gene Enhancer of zeste contains a region with sequence similarity to trithorax. Mol Cell Biol. 1993;13:6357-6366.

Kato J, Matsushime H, Hiebert SW, Ewen ME, Sherr CJ. 1993. Direct binding of cyclin D to the retinoblastoma gene product $(\mathrm{pRb})$ and $\mathrm{pRb}$ phosphorylation by the cyclin $\mathrm{D}$ dependent kinase CDK4. Genes Dev. 1993 Mar;7(3):331-42.

Keller SA, Ullah Z, Buckley MS, Henry RW, Arnosti DN. 2005. Distinct developmental expression of Drosophila retinoblastoma factors. Gene Expr Patterns. 2005 Feb;5(3):411-21.

Khochbin S., Verdel A., Lemercier C., Seigneurin-Berny D. 2001. Functional significance of histone deacetylase diversity. Curr. Opin. Genet. Dev. 11(2): 162-6.

Khorasanizadeh S. 2004. The nucleosome: from genomic organization to genomic regulation. Cell 116(2): 259-72.

Klose RJ and Zhang Y. 2007. Regulation of histone methylation by demethylimination and demethylation. Nat. Rev. Mol. Cell Biol. 8, 307-318.

Kokubo T, Gong DW, Wootton JC. 1994. Molecular cloning of Drosophila TFIID subunits. Nature. 367(6462):484-487.

Koshland D and Strunnikov. 1996. A. Mitotic chromosome condensation. Annu. Rev. Cell Dev. Biol. 12, 305-333.

Kouskouti A, Scheer E, Staub A, Tora L, Talianidis I. 2004. Gene-specific modulation of TAF10 function by SET9-mediated methylation, Mol Cell 14 (2004), pp. 175-182.

Kouzarides T. 2007. Chromatin modifications and their function. Cell 128, 693-705.

Krajewski WA, Nakamura T, Mazo A, Canaani E. 2005. A motif within SET-domain proteins binds single-stranded nucleic acids and transcribed and supercoiled DNAs and can interfere with assembly of nucleosomes. Mol Cell Biol. 2005 Mar;25(5):1891-9.

Krishnamoorthy T, Chen X, Govin J, Cheung WL, Dorsey J, Schindler K, Winter E, Allis CD, Guacci V, Khochbin S, Fuller MT, Berger SL. 2006. Phosphorylation of histone H4 Ser1 regulates sporulation in yeast and is conserved in fly and mouse spermatogenesis. Genes Dev. 2006 Sep 15;20(18):2580-92.

Kuhlmann M, Borisova BE, Kaller M, Larsson P, Stach D, Na J, Eichinger L, Lyko F, Ambros V, Söderbom F, Hammann C, Nellen W. 2005. Silencing of retrotransposons in Dictyostelium by DNA methylation and RNAi. Nucleic Acids Res. 2005;33:6405-6417.

Kunert N, Marhold J, Stanke J, Stach D, Lyko F. 2003. A Dnmt2-like protein mediates DNA methylation in Drosophila. Development. 2003;130:5083-5090.

Kurdistani SK and Grunstein M. 2003. Histone acetylation and deacetylation in yeast. Nat. Rev. Mol. Cell Biol. 4(4): 276-84.

Kuzin B, Tillib S, Sedkov Y, Mizrokhi L, Mazo A. 1994. The Drosophila trithorax gene encodes a chromosomal protein and directly regulates the region-specific homeotic gene fork head. Genes Dev. 1994 Oct 15;8(20):2478-90.

Kwoczynski S. 2002. Funktionale Charakterisierung der Kinase-Aktivität des Transkriptionskoaktivators $\mathrm{TAF}_{\|} 250$ in Drosophila melanogaster. Universität Heidelberg, Inaugural-Dissertation 2002.

Kwon T, Chang JH, Kwak E, Lee CW, Joachimiak A, Kim YC, Lee J, Cho Y. 2003. Mechanism of histone lysine methyl transfer revealed by the structure of SET7/9-AdoMet. EMBO J. 2003 Jan 15;22(2):292-303.

Lachner M, O'Carroll D, Rea S, Mechtler K, Jenuwein T. 2001. Methylation of histone H3 lysine 9 creates a binding site for HP1 proteins. Nature. 2001 Mar 1;410(6824):116-20.

Laemmli UK. 1970. Cleavage of structural proteins during the assembly of the head bacteriophage T4. Nature 227, 680-685.

Lander ES, Linton LM, Birren B, Nusbaum C, Zody MC, Baldwin J, Devon K, Dewar K, Doyle M, FitzHugh W. et al. 2001. Initial sequencing and analysis of the human genome. Nature 409: 860-921. 
Lane ME, Sauer K, Wallace K, Jan YN, Lehner CF, Vaessin H. 1996. Dacapo, a cyclindependent kinase inhibitor, stops cell proliferation during Drosophila development. Cell. 1996 Dec 27;87(7):1225-35.

Lechner MS, Schultz DC, Negorev D, Maul GG, Rauscher FJ 3rd. 2005. The mammalian heterochromatin protein 1 binds diverse nuclear proteins through a common motif that targets the chromoshadow domain. Biochem Biophys Res Commun. 2005 Jun 17;331(4):929-37.

Lee $\mathrm{TI}$ and Young RA. 2000. Transcription of eukaryotic protein-coding genes. Annu. Rev. Genet. 34: 77-137.

Lehnertz B, Ueda Y, Derijck AA, Braunschweig U, Perez-Burgos L, Kubicek S, Chen T, Li E, Jenuwein T, Peters AH. 2003. Suv39h-mediated histone H3 lysine 9 methylation directs DNA methylation to major satellite repeats at pericentric heterochromatin. Curr Biol. 2003 Jul 15;13(14):1192-200.

Lehrke $M$ and Lazar MA. 2005. The many faces of PPARgamma. Cell. 2005 Dec 16;123(6):993-9.

Levinger $L$ and Varshavsky A. 1982. Selective arrangement of ubiquitinated and D1 proteincontaining nucleosomes within the Drosophila genome. Cell 28: 375-385.

Lewis JD, Meehan RR, Henzel WJ, Maurer-Fogy I, Jeppesen P, Klein F and Bird A. 1992. Purification, sequence, and cellular localization of a novel chromosomal protein that binds to methylated DNA, Cell 69 (1992), pp. 905-914.

Li B, Carey M, Workman JL. 2007. The role of chromatin during transcription. Cell 128, 707719.

Li E. 2002. Chromatin modification and epigenetic reprogramming in mammalian development. Nat Rev Genet 3: 662-673.

Li G, Levitus M, Bustamante C, Widom J. 2005. Rapid spontaneous accessibility of nucleosomal DNA. Nat. Struct. Mol. Biol. 12(1): 46-53.

Li H, Rauch T, Chen ZX, Szabó PE, Riggs AD, Pfeifer GP. 2006. The histone methyltransferase SETDB1 and the DNA methyltransferase DNMT3A interact directly and localize to promoters silenced in cancer cells. J Biol Chem. 2006 Jul 14;281(28):19489-500.

Liang G, Chan MF, Tomigahara Y, Tsai YC, Gonzales FA, Li E, Laird PW, Jones PA. 2002. Cooperativity between DNA methyltransferases in the maintenance methylation of repetitive elements. Mol Cell Biol 22: 480-491.

Lomberk G, Wallrath L, Urrutia R. 2006. The Heterochromatin Protein 1 family. Genome Biol. 2006;7(7):228.

Lomvardas S, Thanos D. 2001. Nucleosome sliding via TBP DNA binding in vivo. Cell 106(6): 685-96.

Loyola A. and Almouzni G. 2004. Bromodomains in living cells participate in deciphering the histone code, Trends Cell Biol. 14 pp. 279-281.

Ludlow JW, Glendening CL, Livingston DM, DeCarprio JA. 1993. Specific enzymatic dephosphorylation of the retinoblastoma protein. Mol Cell Biol. 1993 Jan;13(1):367-72.

Lyko F, Ramsahoye BH, Jaenisch R. 2000a. DNA methylation in Drosophila melanogaster. Nature. 2000 Nov 30;408(6812):538-40.

Lyko F, Whittaker AJ, Orr-Weaver TL, Jaenisch R. 2000b. The putative Drosophila methyltransferase gene $d D n m t 2$ is contained in a transposon-like element and is expressed specifically in ovaries. Mech Dev. 2000;95:215-217.

Ma D, Zhou P, Harbour JW. 2003. Distinct mechanisms for regulating the tumor suppressor and antiapoptotic functions of Rb. J Biol Chem. 2003 May 23;278(21):19358-66.

Maile T, Kwoczynski S, Katzenberger RJ, Wassarman DA, Sauer F. 2004. TAF1 activates transcription by phosphorylation of serine 33 in histone H2B. Science. 2004 May 14;304(5673):1010-4. 
Maile T. 2006. Functional characterization of the $\mathrm{COOH}$-terminal kinase activity of the TBPassociated factor TAF1. Dissertation zur Erlangung des Doktorgrades der Naturwissenschaften (Dr. rer. nat.), Universität Hohenheim. 2006.

Marhold J, Kramer K, Kremmer E, Lyko F. 2004. The Drosophila MBD2/3 protein mediates interactions between the MI-2 chromatin complex and CpT/A-methylated DNA. Development. 2004 Dec;131(24):6033-9.

Marmorstein R. 2001. Structure of histone deacetylases: insights into substrate recognition and catalysis. Structure (Camb) 9(12): 1127-33.

Marmorstein R. 2003. Structure of SET domain proteins: a new twist on histone methylation. Trends Biochem Sci. 2003 Feb;28(2):59-62.

Martin C, Zhang Y. 2007. Mechanisms of epigenetic inheritance. Curr Opin Cell Biol. 2007 Jun;19(3):266-72.

Matzke MA, Primig M, Trnovsky J and Matzke AJ. 1989. Reversible methylation and inactivation of marker genes in sequentially transformed tobacco plants, EMBO J. 8 pp. 643-649.

McCallum CM, Comai L, Greene EA and Henikoff S. 2000. Targeted screening for induced mutations. Nat. Biotechnol. 18: 455-457.

Meehan RR, Lewis JD and Bird AP. 1992, Characterization of MeCP2, a vertebrate DNA binding protein with affinity for methylated DNA, Nucleic Acids Res. 20 (1992), pp. 50855092.

Meyer P, Niedenhof I, ten Lohuis M. 1994. Evidence for cytosine methylation of nonsymmetrical sequences in transgenic Petunia hybrida, EMBO J. 13 (1994), pp. 20842088.

Min J, Feng Q, Li Z, Zhang Y, Xu RM. 2003. Structure of the catalytic domain of human DOT1L, a non-SET domain nucleosomal histone methyltransferase. Cell 112(5): 711-23.

Moazed D. 2001. Common themes in mechanisms of gene silencing. Mol. Cell 8(3): 489-98. Review.

Moon H, Filippova G, Loukinov D, Pugacheva E, Chen Q, Smith ST, Munhall A, Grewe B, Bartkuhn M, Arnold R, Burke LJ, Renkawitz-Pohl R, Ohlsson R, Zhou J, Renkawitz $\mathbf{R}$, Lobanenkov V. 2005. CTCF is conserved from Drosophila to humans and confers enhancer blocking of the Fab-8 insulator. EMBO Rep. 2005;6:165-170

Mora-Bermúdez F. 2002. Functional characterization of SET Domain-Containing Proteins in Drosophila melanogaster. Master of Science Thesis. 2002

Morris EJ, Dyson NJ. 2001. Retinoblastoma protein partners. Adv Cancer Res. 2001;82:1-54.

Nakayama J, Rice JC, Strahl BD, Allis CD, Grewal SI. 2001. Role of histone H3 lysine 9 methylation in epigenetic control of heterochromatin assembly. Science. $2001 \mathrm{Apr}$ 6;292(5514):110-3.

Nan X, Campoy $\mathbf{J}$ and Bird A. 1997. MeCP2 is a transcriptional repressor with abundant binding sites in genomic chromatin. Cell 88: 471-481.

Nan X, Meehan RR, Bird A. 1993. Dissection of the methyl-CpG binding domain from the chromosomal protein MeCP2. Nucleic Acids Res. 1993 Oct 25;21(21):4886-4892.

Narlikar G. J., Fan H. Y., Kingston R. E. 2002. Cooperation between complexes that regulate chromatin structure and transcription. Cell 108: 475-87.

Narsa Reddy M, Tang LY, Lee TL, James Shen CK. 2003. A candidate gene for Drosophila genome methylation. Oncogene. 2003 Sep 18;22(40):6301-3.

Neufeld TP, de la Cruz AF, Johnston LA, Edgar BA. 1998. Coordination of growth and cell division in the Drosophila wing. Cell. 1998 Jun 26;93(7):1183-93.

$\mathbf{N g ~ H H}$, Jeppesen P, Bird A. 2000. Active repression of methylated genes by the chromosomal protein MBD1. 2000 Feb;20(4):1394-406. 
Ng HH, Zhang Y, Hendrich B, Johnson CA, Turner BM, Erdjument-Bromage H, Tempst P, Reinberg D, Bird A. 1999. MBD2 is a transcriptional repressor belonging to the MeCP1 histone deacetylase complex. Nat Genet. 1999 Sep;23(1):58-61.

Nichols CD, Crew JR, Batterham P, Pollock JA. 1996. Enhancer trap expression and molecular characterization of the lozenge locus. A. Dros. Res. Conf. $37: 271$.

Nickel BE, Allis CD, and Davie JR. 1989. Ubiquitinated histone H2B is preferentially located in transcriptionally active chromatin. Biochemistry 28: 958-963.

Nielsen SJ, Schneider R, Bauer UM, Bannister AJ, Morrison A, O'Carroll D, Firestein R, Cleary M, Jenuwein T, Herrera RE, Kouzarides T. 2001. Rb targets histone $H 3$ methylation and HP1 to promoters. Nature. 2001 Aug 2;412(6846):561-565.

Nishioka K, Rice JC, Sarma K, Erdjument-Bromage H, Werner J, Wang Y, Chuikov S, Valenzuela P, Tempst P, Steward R, Lis JT, Allis CD, Reinberg D. 2002a. PR-Set7 is a nucleosome-specific methyltransferase that modifies lysine 20 of histone $\mathrm{H} 4$ and is associated with silent chromatin, Mol Cell 9 (2002), pp. 1201-1213.

Nishioka K, Chuikov S, Sarma K, Erdjument-Bromage H, Allis CD, Tempst P and Reinberg D. 2002b. Set9, a novel histone H3 methyltransferase that facilitates transcription by precluding histone tail modifications required for heterochromatin formation, Genes Dev 16 (2002), pp. 479-489.

Nourani A, Utley RT, Allard S, Cote J. 2004. Recruitment of the NuA4 complex poises the PHO5 promoter for chromatin remodeling and activation. EMBO J. 23(13): 2597-607.

Nowak SJ and Corces VG. 2004. Phosphorylation of histone H3: a balancing act between chromosome condensation and transcriptional activation. Trends Genet. 20, 214-220.

Ohki I, Shimotake N, Fujita N, Nakao M, Shirakawa M. 1999. Solution structure of the methylCpG-binding domain of the methylation-dependent transcriptional repressor MBD1. EMBO J. 18, 6653-6661.

Ohki N, Shimotake N, Fujita J, Jee T, Ikegami M, Nakao and Shirakawa M. 2001. Solution structure of the methyl-CpG binding domain of human MBD1 in complex with methylated DNA, Cell 105 (2001), pp. 487-497.

Ohtani K and Nevins JR. 1994. Functional properties of a Drosophila homolog of the E2F1 gene. Mol Cell Biol. 1994 Mar;14(3):1603-12.

Ohtani-Fujita N, Fujita T, Aoike A, Osifchin NE, Robbins PD, Sakai T. 1993. CpG methylation inactivates the promoter activity of the human retinoblastoma tumorsuppressor gene. Oncogene 8, 1063-1067 (1993).

Okano M, Bell DW, Haber DA, Li E. 1999. DNA methyltransferases Dnmt3a and Dnmt3b are essential for de novo methylation and mammalian development. Cell 99: 247-257.

Okano M, Xie S, Li E. 1998. Cloning and characterization of a family of novel mammalian DNA (cytosine-5) methyltransferases. Nat Genet 19: 219-220.

Park JH, Cosgrove MS, Youngman E, Wolberger C, Boeke JD. 2002. A core nucleosome surface crucial for transcriptional silencing. Nat. Genet. 32(2): 273-9.

Pavet V, Quintero C, Cecchini NM, Rosa AL, Alvarez ME. 2006. Arabidopsis displays centromeric DNA hypomethylation and cytological alterations of heterochromatin upon attack by pseudomonas syringae. Mol Plant Microbe Interact. 2006 Jun;19(6):577-87.

Perez-Torrado R, Yamada D, Defossez PA. 2006. Born to bind: the BTB protein-protein interaction domain. Bioessays. 2006 Dec;28(12):1194-202

Peterson CL, Laniel MA, 2004. Histories and histone modifications. Curr Biol. 14(14):R546R551.

Pham AD and Sauer F. 2000. Ubiquitin-activating/conjugating activity of $\mathrm{TAF}_{\|} 250$, a mediator of activation of gene expression in Drosophila. Science 289: 2357-2360.

Pickart CM. 2001. Mechanisms underlying ubiquitination. Annu. Rev. Biochem. 70: 503-533. 
Pitto L, Cernilogar F, Evangelista M, Miarelli C, Lombardi L and Rocchi P. 2000. Characterization of carrot nuclear proteins that exhibit specific binding affinity towards conventional and non-conventional DNA methylation. Plant Mol. Biol. 44:659-673.

Polager S, Kalma Y, Berkovich E, Ginsberg D. 2002. E2Fs up-regulate expression of genes involved in DNA replication, DNA repair and mitosis. Oncogene. 2002 Jan 17;21(3):43746.

Prokhortchouk A, Hendrich B, Jorgensen H, Ruzov A, Wilm M, Georgiev G, Bird A. and Prokhortchouk E. 2001. The p120 catenin partner Kaiso is a DNA methylationdependent transcriptional repressor, Genes Dev. 15 (2001), pp. 1613-1618.

Rai K, Chidester S, Zavala CV, Manos EJ, James SR, Karpf AR, Jones DA, Cairns BR. 2007. Dnmt2 functions in the cytoplasm to promote liver, brain, and retina development in zebrafish. Genes Dev. 2007;21:261-266.

Ramsahoye BH, Biniszkiewicz D, Lyko F, Clark V, Bird AP, Jaenisch R. 2000. Non-CpG methylation is prevalent in embryonic stem cells and may be mediated by DNA methyltransferase 3a. Proc Natl Acad Sci USA 97: 5237-5242.

Rea S, Eisenhaber F, O'Carroll D, Strahl BD, Sun ZW, Schmid M, Opravil S, Mechtler K, Ponting CP, Allis CD, Jenuwein T. 2000. Regulation of chromatin structure by sitespecific histone H3 methyltransferases. Nature. 2000 Aug 10;406(6796):593-9.

Reid JL, lyer VR, Brown PO and Struhl K. 2000. Coordinate regulation of yeast ribosomal protein genes is associated with targeted recruitment of Esa1 histone acetylase. Mol. Cell 6(6): 1297-307.

Reinke $\mathbf{H}$, Horz W. 2003. Histones are first hyperacetylated and then lose contact with the activated PHO5 promoter. Mol. Cell 11(6): 1599-607.

Ren B, Cam H, Takahashi Y, Volkert T, Terragni J, Young RA, Dynlacht BD. 2002. E2F integrates cell cycle progression with $D N A$ repair, replication, and $G(2) / M$ checkpoints. Genes Dev. 2002 Jan 15;16(2):245-56.

Resnitzky D, Hengst L, Reed SI. 1995. Cyclin A-associated kinase activity is rate limiting for entrance into $S$ phase and is negatively regulated in G1 by p27Kip1. Mol Cell Biol. 1995 Aug;15(8):4347-52.

Richards EJ, Elgin SC. 2002. Epigenetic codes for heterochromatin formation and silencing: rounding up the usual suspects. Cell. 108. pp. 489-500.

Ringrose L, Paro R. 2004. Epigenetic regulation of cellular memory by the Polycomb and Trithorax group proteins. Annu Rev Genet. 2004;38:413-43.

Rinn JL, Kertesz M, Wang JK, Squazzo SL, Xu X, Brugmann SA, Goodnough LH, Helms JA, Farnham PJ, Segal E, Chang HY. 2007. Functional demarcation of active and silent chromatin domains in human HOX loci by noncoding RNAs. Cell. 2007 Jun 29;129(7):1311-23.

Robertson KD, Ait-Si-Ali S, Yokochi T, Wade PA, Jones PL, Wolffe AP. 2000. DNMT1 forms a complex with Rb, E2F1 and HDAC1 and represses transcription from E2F-responsive promoters. Nat Genet. 2000 Jul;25(3):338-42.

Robertson KD, Uzvolgyi E, Liang G, Talmadge C, Sumegi J, Gonzales FA and Jones PA. 1999. The human DNA methyltransferases (DNMTs) 1, 3a and 3b: coordinate mRNA expression in normal tissues and overexpression in tumors. Nucleic Acids Res. 27 (1999), pp. 2291-2298.

Roder K, Hung MS, Lee TL, Lin TY, Xiao H, Isobe KI, Juang JL, Shen CJ. 2000. Transcriptional repression by Drosophila methyl-CpG-binding proteins. Mol. Cell. Biol. 20 (2000), pp. 7401-7409.

Ross SE, Hemati N, Longo KA, Bennett CN, Lucas PC, Erickson RL, MacDougald OA. 2000. Inhibition of adipogenesis by Wnt signaling. Science. 2000 Aug 11;289(5481):9503. 
Roth SY, Denu JM, Allis CD. 2001. Histone acetyltransferases. Annu. Rev. Biochem. 70: 81120.

Russo VEA, Martienssen RA, Riggs AD. 1996. Epigenetic Mechanisms of Gene Regulation. Cold Spring Harbor Laboratory Press, Woodbury.

Ruthenburg AJ, Allis CD, Wysocka J. 2007. Methylation of lysine 4 on histone H3: intricacy of writing and reading a single epigenetic mark. Mol Cell. 2007 Jan 12;25(1):15-30.

Ryu H, Lee J, Hagerty SW, Soh BY, McAlpin SE, Cormier KA, Smith KM, Ferrante RJ. 2006. ESET/SETDB1 gene expression and histone H3 (K9) trimethylation in Huntington's disease. Proc Natl Acad Sci U S A. 2006 Dec 12;103(50):19176-81.

Sakai T, Toguchida J, Ohtani N, Yandell DW, Rapaport JM, Dryja TP. 1991. Allele-specific hypermethylation of the retinoblastoma tumor-suppressor gene. Am. J. Hum. Genet. 48, 880-888 (1991).

Salzberg A, Fisher O, Siman-Tov R, Ankri S. 2004. Identification of methylated sequences in genomic DNA of adult Drosophila melanogaster. Biochem Biophys Res Commun. 2004 Sep 17;322(2):465-9.

Santos-Rosa H, Schneider R, Bannister AJ, Sherriff J, Bernstein BE, Emre NC, Schreiber SL, Mellor J, Kouzarides T. 2002. Active genes are tri-methylated at K4 of histone H3. Nature. 2002 Sep 26;419(6905):407-411.

Sarraf SA, Stancheva I. 2004. Methyl-CpG binding protein MBD1 couples histone H3 methylation at lysine 9 by SETDB1 to DNA replication and chromatin assembly. Mol Cell. 2004 Aug 27;15(4):595-605.

Sawado T, Yamaguchi M, Nishimoto Y, Ohno K, Sakaguchi K, Matsukage A. 1998. dE2F2, a novel E2F-family transcription factor in Drosophila melanogaster. Biochem Biophys Res Commun. 1998 Oct 20;251(2):409-15.

Scebba F, Bernacchia G, De Bastiani M, Evangelista M, Cantoni RM, Cella R, Locci MT, Pitto L. 2003. Arabidopsis MBD proteins show different binding specificities and nuclear localization. Plant Mol Biol. 2003 Nov;53(5):715-31.

Schaefer M, Steringer JP, Lyko F. 2008. The Drosophila Cytosine-5 Methyltransferase Dnmt2 Is Associated with the Nuclear Matrix and Can Access DNA during Mitosis. PLoS ONE. 2008 Jan 9;3(1):e1414.

Schneider I. 1972. Cell lines derived from late embryonic stages of Drosophila melanogaster. J. Embryol. Exp. Morphol. 27(2): 353-65.

Schneider $\mathbf{J}$ and Shilatifard A. 2006. Histone demethylation by hydroxylation: chemistry in action. ACS Chem. Biol. 1, 75-81.

Schotta G, Ebert A, Krauss V, Fischer A, Hoffmann J, Rea S, Jenuwein T, Dorn R, Reuter G. 2002. Central role of Drosophila SU(VAR)3-9 in histone H3-K9 methylation and heterochromatic gene silencing. EMBO J. 2002 Mar 1;21(5):1121-31.

Schotta G, Ebert A, Reuter G, 2003. SU(VAR)3-9 is a conserved key function in heterochromatic gene silencing. Genetica. 2003 Mar;117(2-3):149-58.

Schubert HL, Blumenthal RM, Cheng X. 2003. Many paths to methyltransfer: a chronicle of convergence. Trends Biochem Sci. 2003;28:329-335.

Schultz DC, Ayyanathan K, Negorev D, Maul GG, Rauscher FJ 3rd. 2002. SETDB1: a novel KAP-1-associated histone H3, lysine 9-specific methyltransferase that contributes to HP1-mediated silencing of euchromatic genes by KRAB zinc-finger proteins. Genes Dev. 2002 Apr 15;16(8):919-32

Schwabish MA, Struhl K. 2004. Evidence for eviction and rapid deposition of histones upon transcriptional elongation by RNA polymerase II. Mol Cell Biol. 24(23): 10111-7.

Schwartz BE and Ahmad K. 2005. Transcriptional activation triggers deposition and removal of the histone variant H3.3. Genes Dev. 19(7): 804-14. 
Schwendemann A and Lehmann M. 2002. Pipsqueak and GAGA factor act in concert as partners at homeotic and many other loci. Proc Natl Acad Sci U S A. 2002 Oct 1;99(20):12883-8.

Selker EU, Tountas NA, Cross SH, Margolin BS, Murphy JG, Bird AP, Freitag M. 2003. The methylated component of the Neurospora crassa genome. Nature. 2003 Apr 24;422(6934):893-7.

Seum C, Reo E, Peng H, Rauscher FJ 3rd, Spierer P, Bontron S. 2007. Drosophila SETDB1 is required for chromosome 4 silencing. PLoS Genet. 2007 May 11;3(5):e76.

Shi Y, Lan F, Matson C, Mulligan P, Whetstine JR, Cole PA, Casero RA, Shi Y. 2004. Histone demethylation mediated by the nuclear amine oxidase homolog LSD1. Cell. 29;119(7):941-53.

Shi Y. 2007. Histone lysine demethylases: emerging roles in development, physiology and disease. Nat. Rev. Genet. 8, 829-833.

Shilatifard A. 2006. Chromatin modifications by methylation and ubiquitination: implications in the regulation of gene expression. Annu. Rev. Biochem. 75, 243-269.

Shogren-Knaak M, Peterson CL. 2006. Switching on chromatin: mechanistic role of histone H4-K16 acetylation. Cell Cycle. 5(13):1361-5.

Sims RJ III, Nishioka K. \& Reinberg D. 2003. Histone lysine methylation: a signature for chromatin function. Trends Genet. 19, 629-639.

Smallwood A, Estève PO, Pradhan S, Carey M. 2007. Functional cooperation between HP1 and DNMT1 mediates gene silencing. Genes Dev. 2007 May 15;21(10):1169-78.

Smith GE, Ju G, Ericson BL, Moschera J, Lahm HW, Chizzonite R, Summers MD. 1985. Modification and secretion of human interleukin 2 produced in insect cells by a baculovirus expression vector. Proc Natl Acad Sci U S A. 1985 Dec;82(24):8404-8.

Stabell M, Bjørkmo M, Aalen RB, Lambertsson A. 2006. The Drosophila SET domain encoding gene dEset is essential for proper development. Hereditas. 2006 Dec;143(2006):177-88.

Stancheva I. 2005. Caught in conspiracy: cooperation between DNA methylation and histone H3K9 methylation in the establishment and maintenance of heterochromatin. Biochem Cell Biol. 2005 Jun;83(3):385-95.

Stassen MJ, Bailey D, Nelson S, Chinwalla V, Harte PJ. 1995. The Drosophila trithorax proteins contain a novel variant of the nuclear receptor type DNA binding domain and an ancient conserved motif found in other chromosomal proteins. Mech Dev. 1995;52:209223.

Stevaux O, Dimova D, Frolov MV, Taylor-Harding B, Morris E, Dyson N. 2002. Distinct mechanisms of E2F regulation by Drosophila RBF1 and RBF2. EMBO J. 2002 Sep 16;21(18):4927-37.

Stevaux O, Dimova DK, Ji JY, Moon NS, Frolov MV, Dyson NJ. 2005. Retinoblastoma family 2 is required in vivo for the tissue-specific repression of dE2F2 target genes. Cell Cycle. 2005 Sep;4(9):1272-80.

Strahl BD and Allis CD. 2000. The language of covalent histone modifications. Nature 403: 415.

Strunnikova M, Schagdarsurengin U, Kehlen A, Garbe JC, Stampfer MR and Dammann R. 2005. Chromatin inactivation precedes de novo DNA methylation during the progressive epigenetic silencing of the RASSF1A promoter, Mol. Cell. Biol. 25 (2005), pp. 39233933.

Sutcliffe JE, Korenjak M, Brehm A. 2003. Tumour suppressors--a fly's perspective. Eur J Cancer. 2003 Jul;39(10):1355-62.

Szabo P, Tang SH, Rentsendorj A, Pfeifer GP and Mann JR. 2000. Maternal-specific footprints at putative CTCF sites in the $\mathrm{H} 19$ imprinting control region give evidence for insulator function. Curr. Biol. 10: 607-610. 
Tagami H, Ray-Gallet D, Almouzni G, Nakatani Y. 2004. Histone H3.1 and H3.3 complexes mediate nucleosome assembly pathways dependent or independent of DNA synthesis. Cell 116(1): 51-61.

Takada I, Mihara M, Suzawa M, Ohtake F, Kobayashi S, Igarashi M, Youn MY, Takeyama K, Nakamura T, Mezaki Y, Takezawa S, Yogiashi Y, Kitagawa H, Yamada G, Takada S, Minami Y, Shibuya H, Matsumoto K, Kato S. 2007. A histone lysine methyltransferase activated by non-canonical Wnt signalling suppresses PPAR-gamma transactivation. Nat Cell Biol. 2007 Nov;9(11):1273-85.

Takeda S, Paszkowski J. 2006. DNA methylation and epigenetic inheritance during plant gametogenesis. Chromosoma. 2006 Feb;115(1):27-35. Epub 2005 Oct 26.

Talay 0. 2004. Characterization of the histone methyltransferase ASH1 in drosophila melanogaster and its epigenetic activity. Diplom-thesis in Biology (Diplomarbeit), Ruprecht-Karls-Universität Heidelberg. 2004.

Tamaru H and Selker EU. 2001. A histone H3 methyltransferase controls DNA methylation in Neurospora crassa. Nature. 2001 Nov 15;414(6861):277-83.

Tamaru H, Zhang X, McMillen D, Singh PB, Nakayama J, Grewal SI, Allis CD, Cheng X, Selker EU. 2003. Trimethylated lysine 9 of histone H3 is a mark for DNA methylation in Neurospora crassa. Nat Genet. 2003;34:75-79.

Tedesco D, Lukas J, Reed SI. 2002. The pRb-related protein p130 is regulated by phosphorylation-dependent proteolysis via the protein-ubiquitin ligase SCF(Skp2). Genes Dev. 2002 Nov 15;16(22):2946-57.

Thanbichler M, Wang SC, Shapiro L. 2005. The bacterial nucleoid: a highly organized and dynamic structure. J Cell Biochem. 2005 Oct 15;96(3):506-21.

Thiriet C and Hayes JJ. 2005. Replication-independent core histone dynamics at transcriptionally active loci in vivo. Genes Dev. 19(6): 677-82.

Thoma F, Koller T, Klug A. 1979. Involvement of histone $\mathrm{H1}$ in the organization of the nucleosome and of the salt-dependent superstructures of chromatin. J. Cell Biol. 83 (2 Pt 1): 403-27.

Thorne AW, Sautiere P, Briand G and Crane-Robinson C. 1987. The structure of ubiquitinated histone H2B. EMBO J. 6: 1005-1010.

Trievel RC, Flynn EM, Houtz RL, Hurley JH. 2003. Mechanism of multiple lysine methylation by the SET domain enzyme Rubisco LSMT. Nat Struct Biol. 2003 Jul;10(7):545-52.

Tschiersch B, Hofmann A, Krauss V, Dorn R, Korge G, Reuter G. 1994. The protein encoded by the Drosophila position-effect variegation suppressor gene Su(var)3-9 combines domains of antagonistic regulators of homeotic gene complexes. EMBO J. 1994;13:3822-3831.

Tweedie S, Charlton J, Clark V and Bird A. 1997. Methylation of genomes and genes at the invertebrate-vertebrate boundary. Mol. Cell. Biol. 17: 1469-1475

Tweedie S, Ng HH, Barlow AL, Turner BM, Hendrich B, Bird A. 1999. Vestiges of a DNA methylation system in Drosophila melanogaster?. Nat. Genet. 23 (1999), pp. 389-390.

Tzeng TY, Lee CH, Chan LW, Shen CK. 2007. Epigenetic regulation of the Drosophila chromosome 4 by the histone H3K9 methyltransferase dSETDB1.

Vandel L, Nicolas E, Vaute O, Ferreira R, Ait-Si-Ali S, Trouche D. 2001. Transcriptional repression by the retinoblastoma protein through the recruitment of a histone methyltransferase. Mol. Cell. Biol. 21, 6484-6494.

Vaughn JL, Goodwin RH, Tompkins GJ, McCawley P. 1977. The establishment of two cell lines from the insect Spodoptera frugiperda (Lepidoptera; Noctuidae). In Vitro. 1977 Apr;13(4):213-7.

Venter JC, Adams MD, Myers EW, Li PW, Mural RJ, Sutton GG, Smith HO, Yandell M, Evans CA, Holt RA. et al. 2001. The sequence of the human genome. Science 291: 1304-1351. 
Vogelauer M, Wu J, Suka N and Grunstein M. 2000. Global histone acetylation and deacetylation in yeast. Nature 408: 495-8.

Waddington CH. 1957. The Strategy of the Genes. Allen \& Unwin, London,

Wade PA and Wolffe AP. 2001. ReCoGnizing methylated DNA. Nat. Struct. Biol. 8, 575-577.

Wade PA. 2001. Methyl CpG-binding proteins and transcriptional repression, BioEssays 23 (2001), pp. 1131-1137.

Wakefield RI, Smith BO, Nan X, Free A, Soteriou A, Uhrin D, Bird AP, Barlow PN. 1999. The solution structure of the domain from MeCP2 that binds to methylated DNA. J. Mol. Biol. 291, 1055-1065.

Walsh C 2005. Posttranslational Modification of Proteins: Expanding Nature's Inventory. Roberts and Co. Publishers. 2005 October. 1 edition. Chapter 5.

Wan M, Lee SS, Zhang X, Houwink-Manville I, Song HR, Amir RE, Budden S, Naidu S, Pereira JL, Lo IF, Zoghbi HY, Schanen NC, Francke U. 1999. Rett syndrome and beyond: recurrent spontaneous and familial MeCP2 mutations at CpG hotspots, Am. J. Hum. Genet. 65 (1999), pp. 1520-1529.

Wang H, An W, Cao R, Xia L, Erdjument-Bromage H, Chatton B, Tempst P, Roeder RG, Zhang Y. 2003. mAM facilitates conversion by ESET of dimethyl to trimethyl lysine 9 of histone $\mathrm{H} 3$ to cause transcriptional repression. Mol Cell. 2003 Aug;12(2):475-87.

Wang H, Cao R, Xia L, Erdjument-Bromage H, Borchers C, Tempst P and Zhang Y. 2001. Purification and functional characterization of a histone H3-lysine 4-specific methyltransferase, Mol Cell 8 (2001), pp. 1207-1217.

Weinberg RA. 1995. The retinoblastoma protein and cell cycle control. Cell. 1995 May 5;81(3):323-30.

Weissmann F, Muyrers-Chen I, Musch T, Stach D, Wiessler M, Paro R, Lyko F. 2003. DNA hypermethylation in Drosophila melanogaster causes irregular chromosome condensation and dysregulation of epigenetic histone modifications. Mol Cell Biol. 2003 Apr;23(7):2577-86.

Wendt KD \& Shilatifard A. 2006. Packing for the germy: the role of histone H4 Ser1 phosphorylation in chromatin compaction and germ cell development. Genes Dev. 20, 2487-2491.

West MH and Bonner WM. 1980. Histone 2B can be modified by the attachment of ubiquitin. Nucleic Acids Res. 8: 4671-4680.

Wilkinson, KD. 2000. Ubiquitination and deubiquitination: Targeting of proteins for degradation by the proteasome. Semin. Cell. Dev. Biol. 11: 141-148.

Wilson GG and Murray NE. 1991. Restriction and modification systems. Annu Rev Genet. 1991;25:585-627.

Xiao B, Jing C, Kelly G, Walker PA, Muskett FW, Frenkiel TA, Martin SR, Sarma K, Reinberg D, Gamblin SJ, Wilson JR. 2005. Specificity and mechanism of the histone methyltransferase Pr-Set7, Genes Dev 19 (2005), pp. 1444-1454.

Xiao B, Jing C, Wilson JR, Walker PA, Vasisht N, Kelly G, Howell S, Taylor IA, Blackburn GM, Gamblin SJ. 2003b. Structure and catalytic mechanism of the human histone methyltransferase SET7/9. Nature. 2003 Feb 6;421(6923):652-6.

Xiao B, Wilson JR, Gamblin SJ. 2003a. SET domains and histone methylation. Curr Opin Struct Biol. 2003 Dec;13(6):699-705.

Xin S, Weng L, Xu J, Du W. 2002. The role of RBF in developmentally regulated cell proliferation in the eye disc and in Cyclin D/Cdk4 induced cellular growth. Development. 2002 Mar;129(6):1345-56.

Xin Z, Tachibana M, Guggiari M, Heard E, Shinkai Y, Wagstaff J. 2003. Role of histone methyltransferase G9a in CpG methylation of the Prader-Willi syndrome imprinting center. J Biol Chem. 2003;278:14996-15000. 
Xing Y, Shi S, Le L, Lee CA, Silver-Morse L, Li WX. 2007. Evidence for transgenerational transmission of epigenetic tumor susceptibility in Drosophila. PLoS Genet. 2007 Sep;3(9):1598-606.

Yang L, Xia L, Wu DY, Wang H, Chansky HA, Schubach WH, Hickstein DD, Zhang Y. 2002. Molecular cloning of ESET, a novel histone H3-specific methyltransferase that interacts with ERG transcription factor Oncogene. 2002 Jan 3;21(1):148-52.

Yang XJ. 2004. Lysine acetylation and the bromodomain: a new partnership for signaling, Bioessays 26 pp. 1076-1087.

Yen RW, Vertino PM, Nelkin BD, Yu JJ, W. el-Deiry, Cumaraswamy A, Lennon GG, Trask BJ, Celano P, Baylin SB. 1992. Isolation and characterization of the cDNA encoding human DNA methyltransferase. Nucleic Acids Res. 20 (1992), pp. 2287-2291.

Yoon J, Lee KS, Park JS, Yu K, Paik SG, Kang YK. 2008. dSETDB1 and SU(VAR)3-9 sequentially function during germline-stem cell differentiation in Drosophila melanogaster. PLoS ONE. 2008 May 21;3(5):e2234.

Zemach A, Li Y, Wayburn B, Ben-Meir H, Kiss V, Avivi Y, Kalchenko V, Jacobsen SE, Grafi G. 2005. DDM1 binds Arabidopsis methyl-CpG binding domain proteins and affects their subnuclear localization, Plant Cell 17 (2005), pp. 1549-1558.

Zemach A. and G. Grafi G. 2003. Characterization of Arabidopsis thaliana methyl-CpG-binding domain (MBD) proteins, Plant J. 34 (2003), pp. 565-572.

Zeng L, and Zhou MM. 2002. Bromodomain: an acetyl-lysine binding domain, FEBS Lett. 513 pp. 124-128.

Zhang DL, Ehrlich KC, Supakar PC, Ehrlich M. 1989. A plant DNA-binding protein that recognizes 5-methylcytosine residues. Mol. Cell Biol. 9: 1351-1356.

Zhang HS, Gavin M, Dahiya A, Postigo AA, Ma D, Luo RX, Harbour JW, Dean DC. 2000. Exit from $\mathrm{G} 1$ and $S$ phase of the cell cycle is regulated by repressor complexes containing HDAC-Rb-hSWI/SNF and Rb-hSWI/SNF. Cell. 2000 Mar 31;101(1):79-89.

Zhang L, Eugeni EE, Parthun MR, Freitas MA. 2003a. Identification of novel histone posttranslational modifications by peptide mass fingerprinting. Chromosoma 112(2): 77-86.

Zhang X, Yang Z, Khan SI, Horton JR, Tamaru H, Selker EU, Cheng X. 2003b. Structural basis for the product specificity of histone lysine methyltransferases. Mol Cell. 2003;12:177-185.

Zhang $\mathbf{Y}$ and Reinberg D. 2001. Transcription regulation by histone methylation: interplay between different covalent modifications of the core histone tails. Genes Dev. 15(18): 2343-60.

Zhang Y. 2003c. Transcriptional regulation by histone ubiquitination and deubiquitination. Genes Dev. 17, 2733-2740.

Zheng C, Hayes JJ. 2003. Structures and interactions of the core histone tail domains. Biopolymers. 68(4):539-546.

Zhou J, Chau C, Deng Z, Stedman W, Lieberman PM. 2005. Epigenetic control of replication origins. Cell Cycle. 4(7):889-92. 


\section{Acknowledgements}

I would like to express my earnest thanks and gratitude to my advisors Dr. Frank Sauer and Dr. Ernst Wimmer for support, guidance, and encouragement during my graduate study. My sincere thanks and appreciation go to Dr. Gerhard Braus and Dr. Michale Kessel for their discussion, advice and support over the years.

I would like to thank my co-workers Tilmann-Sanchez Elstner, Tobias Maile, Silvia Sauer, Ninuo Xia, Flora (Pei-Fang Tsai), Monica Rubalcava, Joshua Lee Halford, Alice Kelly Kan, Oezcan Talay, Julia Sommer, Juanma (Juan Manuel Caravaca), Stéphane Bertani, David Parker, Nomiki Kolettis and Anita Pavitrin for their continual help. My sincere thanks go to Alex (Alexandra Kötter) for her help in many aspects from ordering, lab maintaining to translating my files of promotion.

I'm very grateful to Dr. Ernest Martinez and Dr. Xuan Liu for using their laboratory equipments to pursue my thesis.

Much appreciation goes to my parents and my brother for their support. My sincere thanks go to my wife, Haibo Xu and my daughter April, who gave me love, courage and inspired me in many ways.

This work is dedicated to my dear wife. 


\section{Curriculum Vitae}

\section{Personal Data}

Name: Dawei Gou

Date of Birth: June 7, 1974

Place of Birth: Xingcheng, Liaoning Province, P. R. China

\section{Education}

- Candidate of Dr. rer. nat.

April 2005 to date, Institute of Zoology, Anthropology and Developmental Biology, Georg August University, Göttingen

- Advisor: Dr. Ernst Wimmer, Professor of Georg August University, Göttingen

- Co-advisor: Dr. Frank Sauer, Associate Professor of University of California, Riverside

- Master in Biochemistry \& Molecular Biology

September 1998 to July, 2001, Institute of Basic Medical Sciences, Peking Union Medical College (PUMC)

- Advisor: Professor Linfang Wang, Director, National Laboratory of Medical Molecular Biology. Academician, China academy of engineering

- Co-advisor: Professor Shiying Miao, Vice Director, National Laboratory of Medical Molecular Biology

- Bachelor of Clinical and Basic Medical Sciences

September 1992 to July 1998, China Medical University

\section{Laboratory experiences}

- February 2003 to date: first Postgraduate researcher, then Junior Specialist and now Assistant Specialist, Laboratory of Frank Sauer, Biochemistry Department, University of California, Riverside - June 2002 to January 2003: Wissenchaftlicher Mitaarbeiter, Laboratory of Frank Sauer, ZMBH, University of Heidelberg

- July 2001 to May 2002, Lab Assistant, Laboratory of Linfang Wang, National Laboratory of Medical Molecular Biology, Peking Union Medical College (PUMC)

\section{Publications}

- Tilman Sanchez-Elsner, Dawei Gou, Elisabeth Kremmer and Frank Sauer. Noncoding RNAs of trithorax response elements recruit Drosophila Ash1 to Ultrabithorax. Science. 2006 Feb 24;311 (5764):1118-23.

- Xiaodong Zhang, Dawei Gou, Shiying Miao, Jianchao Zhang, Shudong Zhong, Linfang Wang. Cloning and Characterization of a Novel Rat Gene RSD-7 Differentially Expressed in Testis, ACTA ACADEMIAE MEDICINAE SINICAE (Zhongguo Yi Xue Ke Xue Yuan Xue Bao), 2003 Vol.25 No.3 P.289-293. 